
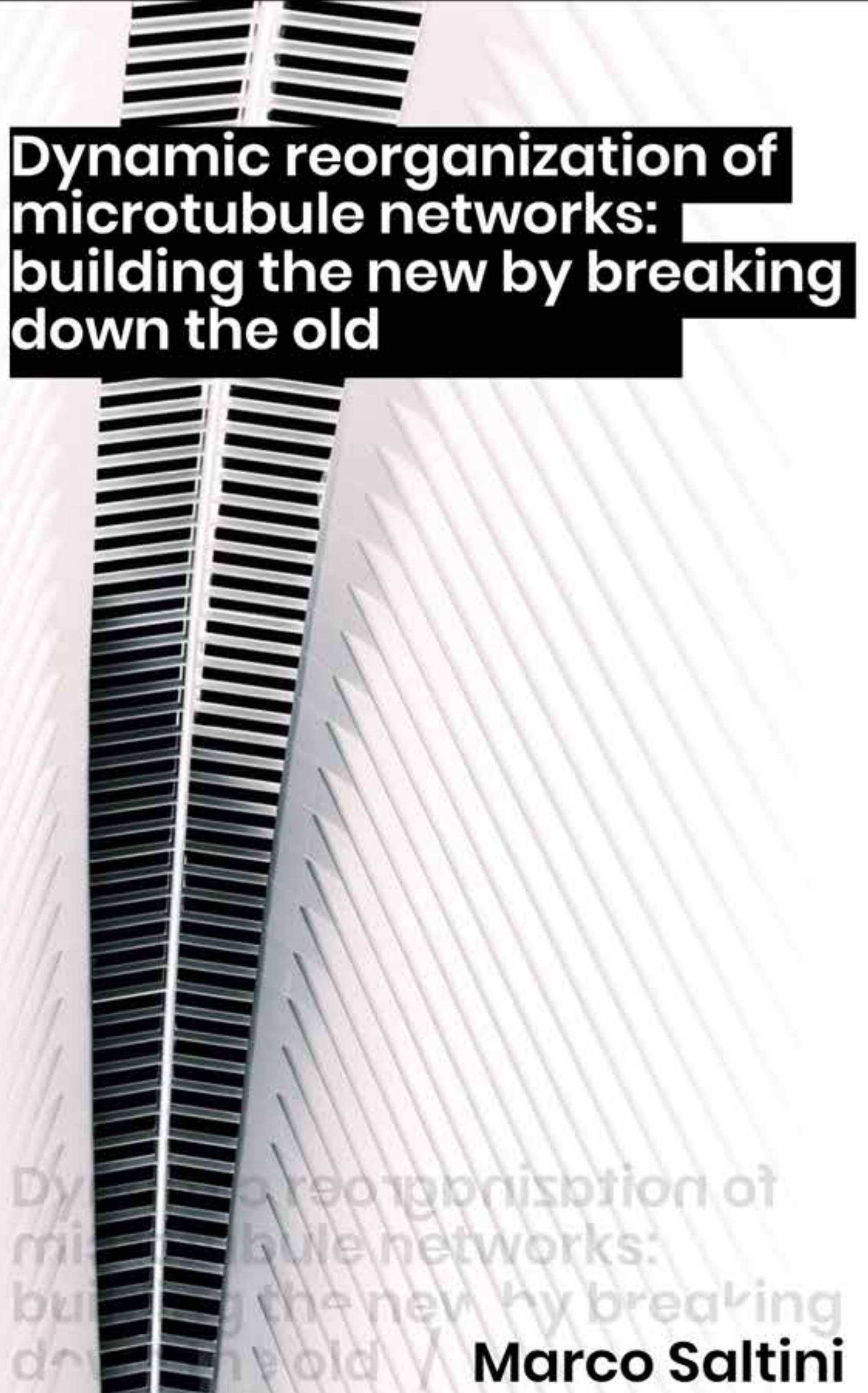


\section{Dynamic reorganization of microtubule networks: building the new by breaking down the old}

Marco Saltini 


\section{Thesis committee}

\section{Promotor}

Prof. Dr B.M. Mulder

Special Professor, Theoretical Cell Physics

Wageningen University \& Research

Group leader Theory of Biomolecular Matter

AMOLF, Amsterdam

\section{Other members}

Prof. Dr J. Molenaar, Wageningen University \& Research Prof. Dr M. Dogterom, Delft University of Technology

Prof. Dr L.C. Kapitein, Utrecht University

Dr F. Nédélec, University of Cambridge, United Kingdom 


\title{
Dynamic reorganization of microtubule networks: building the new by breaking down the old
}

\author{
Marco Saltini
}

Thesis

submitted in fulfilment of the requirements for the degree of doctor at Wageningen University

by the authority of the Rector Magnificus,

Prof. Dr A.P.J. Mol,

in the presence of the

Thesis Committee appointed by the Academic Board

to be defended in public

on Monday 2 December 2019

at 11 a.m. in the Aula. 


\section{Marco Saltini}

Dynamic reorganization of microtubule networks: building the new by breaking down the old, 180 pages.

PhD thesis, Wageningen University, Wageningen, The Netherlands (2019)

With references, with summary in English

ISBN: 978-94-6395-124-1

DOI: https://doi.org/10.18174/500414 
1 Introduction $\quad \mathbf{1}$

1.1 Microtubules are dynamic polymers of the cytoskeleton . . . . . 3

1.2 Research questions . . . . . . . . . . . . . . . . 7

1.3 Modelling microtubule dynamics . . . . . . . . . . . . . . . . . . 9

1.4 Outline .............................. 12

2 CLASP and SPR2 promote microtubule reorientation by acting as tip regulatory factors

2.1 The cortical microtubule array undergoes a katanin-mediated reorientation .................... 16

2.2 CLASP stabilizes newly-created plus ends after severing . . . . . . 22

2.3 SPR2 protects minus end from fast depolymerization . . . . . . . . 29

2.4 Minus end dynamic instability changes steady-state properties of microtubules . . . . . . . . . . . . . . . 36

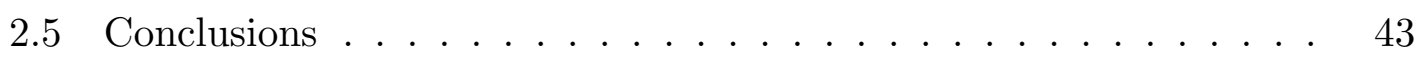

3 Probability of rescue after severing defines a critical threshold for microtubule amplification $\quad 45$

3.1 The model . . . . . . . . . . . . . . . . . . . . . 46

3.2 Results . . . . . . . . . . . . . . . . . . . . . 49

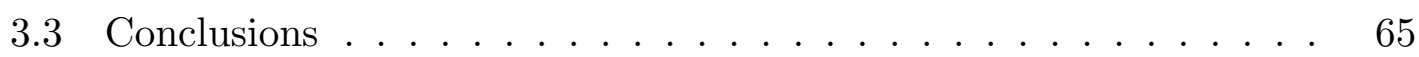

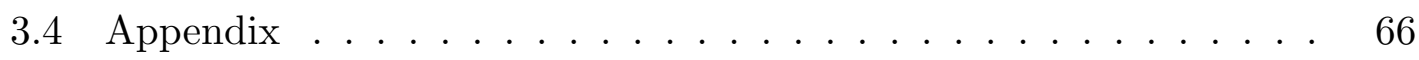


4 Reorientation of the cortical microtubule array in presence of finite tubulin pool

4.1 Preferential severing of longitudinal microtubules does not explain a maintained reorientation . . . . . . . . . . . . 72

4.2 Small difference in microtubule growth speed maintains the reorientation of the array . . . . . . . . . . . . 87

4.3 Computational approach . . . . . . . . . . . . . . . . . . 97

4.4 Conclusions . . . . . . . . . . . . . . . . . . . . . . 102

5 Microtubule-based actin transport in a three-dimensional confinement

5.1 Overview of experiments . . . . . . . . . . . . 106

5.2 The model: dynamic microtubules and diffusing actin . . . . . . 110

5.3 Fast diffusion limit: an analytical solution . . . . . . . . . . . . . . 118

5.4 Fast transport localizes actin at the surface . . . . . . . . . . . . 129

5.5 Conclusions . . . . . . . . . . . . . . . . . 138

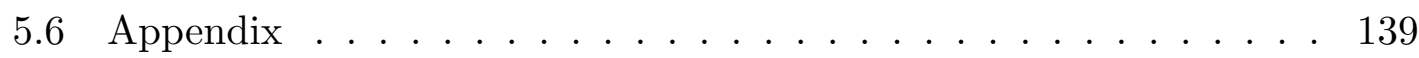

$\begin{array}{lr}\text { Conclusions } & 140\end{array}$

Appendix A Probability distribution for a multi-state ageing $\begin{array}{lr}\text { process } & 153\end{array}$

A.1 The model . . . . . . . . . . . . . . . . . . 153

A.2 Age distribution . . . . . . . . . . . . . . 155

A.3 Age probability . . . . . . . . . . . . . . 157

$\begin{array}{ll}\text { Bibliography } & 161\end{array}$

$\begin{array}{lr}\text { English summary } & 168\end{array}$

$\begin{array}{ll}\text { Acknowledgments } & \mathbf{1 7 0}\end{array}$

$\begin{array}{ll}\text { List of publications } & 173\end{array}$ 


\section{CHAPTER 1}

\section{Introduction}

Looking at the natural world around us, it is impossible not to be impressed by the brilliance and the richness of its complexity. Starting from macroscopic patterns such as "fir waves" in mountain forests (Sprugel, J. Ecol., 1976) or "tiger bushes" in arid regions (Tongway et al., 2001), to the existence of complex life forms as plants and animals, where billions of billions of cells operate and interact to sustain the organism, all of these system share the same property. Indeed they are all highly composite, with components that are in principle "designed" to execute simple tasks, and cooperate and interact to give rise to a wide range of physical properties such as self-organization, non-linearity, pattern formation, among others.

Systems with a high degree of complexity are everywhere in the world, and at any length scale. As an example, the ecosystem of the tropical rainforest is composed of very many different species of animals and plants that constantly interact with each other. The way all different species with their behaviour play a role in sustaining the forest is surprising, as at first sight the property of a single species might not be thought to have any effect on such a large ecosystem, whereas the extinction of a single species can irreparably compromise this robust, yet fragile because of human activity, system. A large flying fox from the forest, e.g., does not know what is necessary for the tropical forest in order to survive, but it only knows what is necessary for itself to survive. Nevertheless, what the rainforest needs the large flying fox species to do in order to contribute to the forest survival, is exactly 
what that species does. For example, flying foxes disperse seeds while flying, and because of their large home ranges, can disperse seeds in wide areas (Oleksy et al., Plos One, 2017). This mechanisms is crucial to promote the diversity of the forest (Harms et al., Nature, 2000). More generally, all the plants and animals of the rainforest cooperate and interact in a way that preserves the latter, while being unaware of that.

Different approaches can be used to study the rainforest or any other complex system. Among those, one option is mathematical modelling, that allows us to represent the system in an idealized and simple way, including only the features that we are interested in studying. Depending on the system under study, different mathematical approaches could be used. For example, for large systems that change on a long time scale such as the rain forest, one could, in principle, write down a set of millions of deterministic coupled differential equations, one for each component of the system. It is clear that this approach is not efficient because, even in the eventuality that the set of equations was solvable, its solution would require an extraordinary computational power that, at the moment, we lack.

For systems changing on shorter spatial and temporal scales such as the cell, instead, some mechanisms are intrinsically stochastic. Microtubules, a complex network of dynamic polymers and components of the cytoskeleton of eukaryotic cells, for example, exhibit a stochastic behaviour as they alternate periods of growth and periods of shrinkage, with growing and shrinking time that cannot be deterministically predicted. Indeed, their growing and shrinking time obeys well-defined probabilistic laws, and therefore one can only predict the average quantity, not the time of a single event.

In both cases, it follows that stochastic modelling should be used to better model these systems. In the first scenario, with large complex systems, it can be used for example to approximate the effect caused on the system by the dynamics of many of its components as if they were just a random noise that affects the single species we are interested in studying. In the second case, instead, stochastic modelling not only can better describe all the interactions between different components in terms of probability of interaction, but it also accounts the intrinsic stochastic properties of the system components.

In this thesis, we are going to study one of these complex systems with this stochastic modelling approach. More specifically, we are going to apply the theory of stochastic processes to the dynamics of the already mentioned microtubules. We will first see how a specific class of microtubules in plants, the cortical microtubule array, changes its orientation and maintains it as a consequence of the interaction between individual microtubules. This process is very important for the anisotropic cell expansion, and ultimately for the plant morphogenesis. Later in the thesis we will make a step further by taking a look at how microtubules interact with another 
polymer component of the cytoskeleton, i.e. actin, and we will make predictions about the consequences of such an interaction.

\subsection{Microtubules are dynamic polymers of the cytoskeleton}

In this section, we introduce the main characters of this thesis, the microtubules. We highlight some of their biological peculiarities, in particularly those that motivate our modelling choices in the remaining chapters.

\section{The cytoskeleton}

The cytoskeleton is a complex, dynamic network present in the cells of all domains. It has multiple and various functions, functional to the type of cell and organisms, but the most common and important ones are: to give strength to the cell, to give shape to the cell, to form the mytotic spindle, among others (Alberts, 2002).

In the eukaryotic domain, besides a multitude of linking and associated proteins, cytoskeleton is composed by three distinct polymers: microtubules, actin filaments, and intermediate filaments. Microtubules, in particular, are important for many cellular mechanisms. Indeed they play an active role in mechanisms such as gene expression, cell migration, and mitosis (Walczak \& Heald, Int. Rev. Cytol., 2008). Moreover, microtubules are involved in the development and in the morphogenesis of the cells, and hence in the morphogenesis of the organisms (Lindeboom et al., Science, 2013). In this thesis, in particular, we will focus on the role of the plant cortical microtubule array in the morphogenesis of higher plants.

\section{The cortical microtubule array}

The cortical microtubule array is an acentrosomal cytoskeletal structure in plant cells located in a thin layer of the cytoplasm and bounded to the plasma membrane. It consists of relatively short dynamic microtubules, the spatial organization of which is very important for the control of cell expansion (Dixit \& Cyr, Plant Cell, 2004). As an example, a rapidly elongating cell exhibits a very ordered structure in which all cortical microtubules are bundled in a transverse direction to the direction of the growth of the cell, see Figure 1.1, while they reorient to a direction longitudinal to the growth direction of the cell when the latter elongates more slowly (Sugimoto et al., Protoplasma, 2001). Experiments have revealed that the reorganization of the cortical microtubule array can be triggered by environmental 
and hormonal signals (Sambade et al., Plant Cell, 2012 - Vineyard et al., Plant Cell, 2013 - Lindeboom et al., Science, 2013).

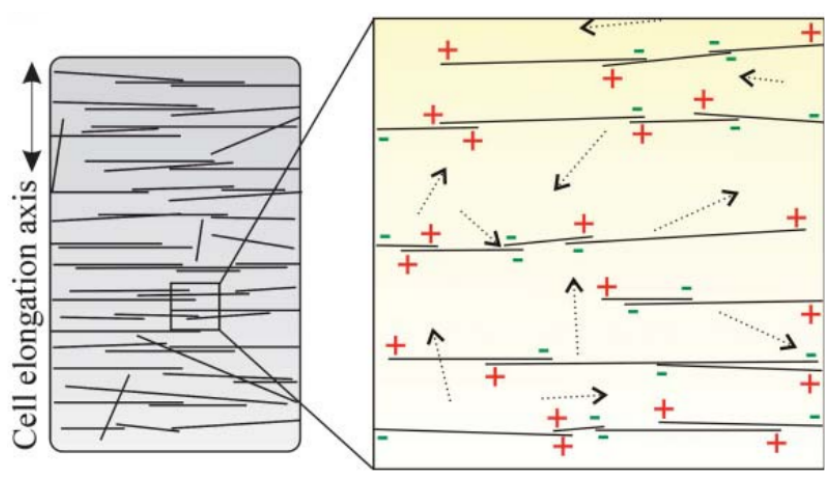

Figure 1.1: Cortical microtubules in a rapidly elongating plant cell. Image is taken from (Dixit \& Cyr, Plant Cell, 2004)

\section{Structure of microtubules}

The elementary unit of a microtubule is a heterodimer of two tubulin proteins: $\alpha$-tubulin and $\beta$-tubulin. These proteins are arranged to form an end-to-tail protofilament. Usually, microtubules consist of 13 of these protofilaments, organized in a tubular conformation, see Figure 1.2. However, it is not uncommon to find microtubules composed by a different number of protofilaments (Chrétien et al., J. Cell Biol., 1992).

The stacking of the tubulin dimers with $\alpha-\beta$ subunit binding is such that they all have the same orientation. Specifically, all $\beta$-proteins are directed towards the most dynamically unstable among the two microtubule tips, the so-called plus end, while $\alpha$-tubulin is directed towards the more stable tip, the so-called minus end (Desai \& Mitchison, Annu. Rev. Cell Dev. Biol., 1997).

Despite its small inner and outer diameters, $12 \mathrm{~nm}$ and $24 \mathrm{~nm}$ respectively, the typical length of a microtubule is on the order of tens of micrometers, whilst its persistence length is about 1-2 mm (Ledbetter \& Porter, Science, 1964); therefore, they can be seen as almost one dimensional stiff structures.

\section{Dynamic instability}

As we introduced already in the previous sections, microtubules are very dynamic polymers, the dynamics of which mainly occurs at the plus end. This is a consequence of the polarity induced by the $\alpha$ - $\beta$ stacking. They spend part of their 


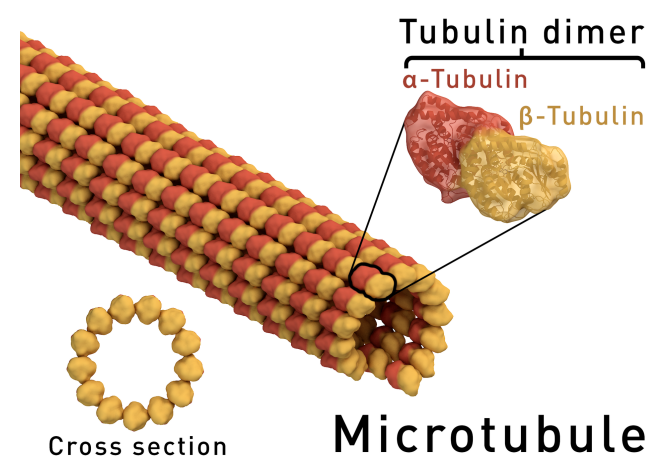

Figure 1.2: Structure of a microtubule. Courtesy of Thomas Splettstoesser (www.scistyle.com).

lifetime in the polymerizing phase (growing state) and part in the depolymerizing phase (shrinking state), with the possibility of switching from one state to the other with the so-called catastrophes - growing-to-shrinking, and rescues - shrinkingto-growing, see Figure 1.3. Usually, the growing and the shrinking speeds are approximately constant during the growth and shrinkage period, respectively.

Polymerization of microtubules is made possible by GTP that binds to $\beta$ tubulin at microtubule tips, stabilizes the tip, and promotes the attachment of new tubulin dimers. However, the GTP cap bound to $\beta$-tubulin is hydrolized to GDP shortly after the creation of the bond. This results in a reduction of affinity between neighbour tubulin proteins that causes destabilization and, therefore, depolymerization and shrinkage of the microtubule. In other words, the GTP creates a protective cap against subunit loss at the tip of the microtubule, while hydrolyisis of GTP to GDP antagonizes this protective effect (Mitchinson, Nature 1984). Hence, whether the microtubule persists in polymerizing or it undergoes a catastrophe and depolymerizes depends on the relation between the binding rate of new dimers with GTP caps and the hydrolyzing rate of GTP to GDP.

\section{Nucleation}

Microtubule nucleation is the process that initiates a new microtubule starting from separate tubulin dimers. Besides the $\alpha$ and $\beta$, a third kind of tubulin, mainly associated to microtubule nucleation, is $\gamma$-tubulin. Most of $\gamma$-tubulin is organized in ring complexes, the so-called $\gamma$-TuRC in the Microtubule Organizing Centre (hereafter MTOC) of animal cells, where it acts as a cap for microtubule minus end to stabilize the latter while the plus end undergoes polymerization, enabling in this way the nucleation of a new microtubule (Kollman et al., Nature, 2010). 


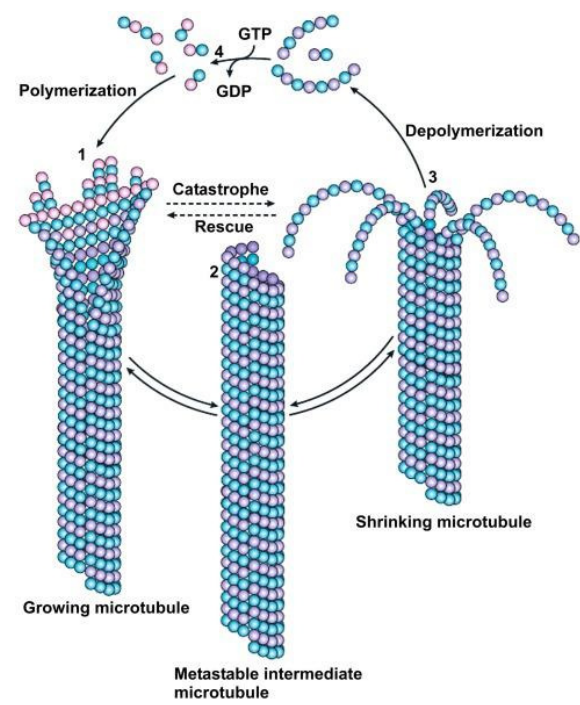

Figure 1.3: Schematic representation of a microtubule undergoing dynamic instability. Image is taken from (Draber et al., Front Immunol. 2012)

Plant cells, however, lack MTOCs. Still, they form complex microtubule structures as the cortical microtubule array. Here, in contrast with the animal case, $\gamma$-tubulin is not localized at a specific site, but it is distributed anywhere in the cell (Ehrhardt et al., J. Cell Sci., 2002). It has been shown that, although $\gamma$-tubulin can be dispersed in the cytosol and hence initiates microtubules from dispersed sites (Schmit, Int. Rev. Cyt., 2002), most part of it binds to the lattice of already existing microtubules, suggesting that the nucleation of new microtubules mainly occurs from already existing ones (Murata et al., Nat. Cell Biol., 2005 - Ehrhardt, Curr. Opin. Cell Biol., 2008). We refer to the nucleation from dispersed sites as bulk or dispersed nucleation, while we refer to the microtubule-based nucleation as bound or branching nucleation.

\section{Microtubule Associated Proteins}

Microtubule Associated Proteins (MAPs) are proteins that interact with microtubules by binding to tubulin dimers and regulate microtubule dynamics (Desai \& Mitchison, Annu. Rev. Cell Dev. Biol., 1997). A large number of MAPs with different functions and different localizations on the microtubule lattice have been identified in the last three decades. Their function varies from stabilizing or destabilizing microtubules, to mediating microtubule interaction with other proteins (Maccioni \& Cambiazo, Physiol. Rev., 1995). In this thesis we will focus 
on a few MAPs of three different kinds: plus end tracking proteins such as CLASP, $\mathrm{EB}$, or SPIRAL1, minus end tracking proteins as SPIRAL2, and severing proteins as katanin, and we will discuss how their function is intimately linked to the spatial organization of the cortical microtubule array.

\section{Katanin}

Named after the Japanese sword katana, katanin is an ATPase associated protein, one of the functions of which is to sever microtubules along their lattice. A severing event at the microtubule lattice initiates a new microtubule, as the mother is divided in two daughters, independently on the presence of a $\gamma$ complex. The effect of katanin on the creation of new microtubules and on the dynamics of the cortical array will be discussed in details in Chapters 2, 3, and 4.

Structurally, katanin is a heterodimeric protein consisting of a first $60 \mathrm{kDa}$ ATPase subunit that requires ATP hydrolysis to sever microtubules, and a second 80 $\mathrm{kDa}$ subunit that regulates the first subunit and localizes at the MTOCs (McNally \& Vale, Cell, 1993).

Katanin severs microtubules in multiple steps: firstly, it localizes at the microtubule lattice and forms an hexomeric complex ring. Then ATP hydrolysis starts, and when all phospates have been released, katanin undergoes conformational changes that can disrupt tubulin-tubulin contacts (Hartman \& Vale, Science, 1999 - Quarmby, J Cell Sci, 2000).

\section{$1.2 \quad$ Research questions}

In this thesis we are going to study how the dynamics of cytoskeletal components influences the spatial organization of the cytoskeleton. In particular, we are going to discuss two mechanisms: the reorientation of the cortical microtubule array (Chapters 2, 3, and 4), and the transport of actin by the interaction with microtubules (Chapter 5).

Cortical microtubules are very important for plant morphogenesis as they play an active role in rapidly elongating the cell by orienting transversely to the growth direction of the cell axis. In this way, they transport the building material for the cell cortex to expand (Elliot \& Shaw, Plant Physiol., 2018). Experiments revealed that, as soon as the elongation rate of the cell slows down, cortical microtubules spatially reorganize. They indeed change their direction from transverse to longitudinal to the direction of elongation of the cell (Lindeboom et al., Science, 2013). Given the absence of MTOCs in plant cells, one can hypothesize that cortical microtubules self-reorganize from the old to the new spatial arrangement. As we are in presence 
of a self-organizing system composed by a large number of identical components, we can argue that the cortical microtubule array is a complex system. Furthermore, microtubules undergo intrinsic stochastic dynamics, with repetitions of catastrophes and rescues. The occurrence of a catastrophe after a rescue event (or viceversa), can be modelled as a Poisson-like event (Verde et al., J. Cell Biol., 1992). Therefore, we choose to approach the study of the reorientation mechanism with stochastic modelling. Thus, in the first part of the thesis, we will set up different stochastic models of dynamic microtubules aimed at addressing two questions:

- Which factors play a role in the reorientation of the cortical array?

- How is the reoriented array maintained in the new direction?

In the second part of the thesis, we will study the spatial organization of the cytoskeleton when only two of its components are present: actin filaments and microtubules. Historically, the two different filaments have been extensively studied, but only independently from each other. However, the importance of their interaction has been acknowledged already sixty years ago (Abercrombie, Exp. Cell. Res., 1961 - Vasiliev et al., Development, 1970). Indeed, in order to develop, the cell needs to control the interaction between different cytoskeletal components (Adikes et al., J. Cell Biol., 2017). Furthermore, the interaction between actin and microtubules is thought to be crucial for neuronal growth, cell migration and division, cellular wound healing, among others (Tortosa et al., J. Biol. Chem., 2011 - Wu et al., Cell, 2008 - Huber et al., Curr. Opin. Cell. Biol., 2015 - Dogterom \& Koenderink, Nat. Rev. Mol. Cell Biol., 2018). Recently performed experiments (Alkemade et al., in preparation) showed that the interaction between actin filaments and microtubules mediated by the crosslinking protein TipAct has a double effect: it destabilizes microtubules by changing their dynamic properties, and makes the actin filaments be transported by the plus end of growing microtubules. In this work, starting from the experimental observations, we will set up a stochastic model of microtubules undergoing dynamic instability in three-dimensional confinement where actin filaments can freely diffuse. We will address the questions:

- How is the spatial organization of actin and microtubules changed by their interaction with respect to the corresponding non-interacting system?

- How can we control the spatial organization by changing the dynamic properties of actin filaments and microtubules? 


\subsection{Modelling microtubule dynamics}

Among the possible stochastic models for microtubule dynamics, the most canonical one is the Dogterom-Leibler model (Dogterom \& Leibler, Phys. Rev. Lett., 1993). This model describes microtubule dynamics on the length scale of micrometers and on the time scale of seconds. This means that the addition or the loss of a single - or a small group, of tubulin dimers to the already existing microtubules is not taken into account. On the contrary, the model considers the average growth or shrinkage of the length of a microtubule in a certain unit of time. Thermal fluctuations that can locally change the growth and shrinkage rate are neglected in this model.

\section{The model}

In this model, a microtubule is modeled as a straight line either in growing state $(+)$, when it increases its length with growing speed $v^{+}$, or shrinking state $(-)$, when the length decreases with shrinking speed $v^{-}$. The microtubule can switch between the two states with a certain catastrophe rate $r_{c}$, and rescue rate $r_{r}$, and new microtubules are nucleated with nucleation rate $r_{n}$, see Figure 1.4.

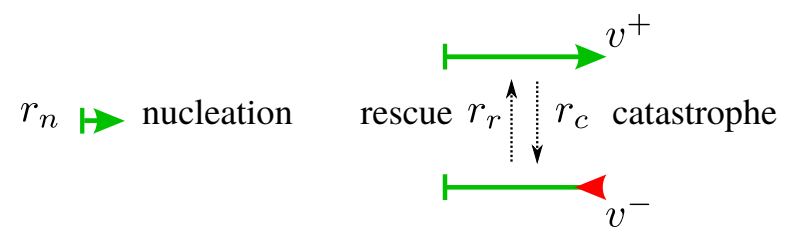

Figure 1.4: Schematic representation of Dogterom-Leibler model of microtubules undergoing dynamic instability.

Therefore, let $m^{ \pm}(t, l)$ be the probability distribution of growing/shrinking microtubules with length $l$ at time $t$. Then, dynamic equations for these two distributions are

$$
\frac{\partial}{\partial t} m^{+}(t, l)=-v^{+} \frac{\partial}{\partial l} m^{+}(t, l)-r_{c} m^{+}(t, l)+r_{r} m^{-}(t, l),
$$

and

$$
\frac{\partial}{\partial t} m^{-}(t, l)=v^{-} \frac{\partial}{\partial l} m^{-}(t, l)-r_{r} m^{-}(t, l)+r_{c} m^{+}(t, l),
$$

with boundary conditions

$$
v^{+} m^{+}(t, 0)=r_{n},
$$




$$
\lim _{l \rightarrow+\infty} m^{ \pm}(t, l)=0 .
$$

Eqs. (1.1) and (1.2) are first order coupled partial differential equations, and are 1-dimensional transport equations with source and decay, due to the switch from one state to the other. The boundary conditions account the fact that all new microtubules are nucleated in the growing state with initial length $l=0$, and the properties of probability functions of approaching 0 at $l \rightarrow \infty$.

We can easily convince ourselves that the average growth length for a microtubule from a rescue to the first catastrophe is $l^{+}=v^{+} / r_{c}$, while the average shrinkage length for a shrinking microtubule from a catastrophe to the following rescue is $l^{-}=v^{-} / r_{r}$. In particular,the model predicts the existence of two distinct regimes of growth for the microtubules: the bounded-growth regime, i.e. when $l^{-}>l^{+}$, and the unbounded-growth regime, when $l^{+}>l^{-}$(Dogterom \& Leibler, Phys. Rev. Lett., 1993). The typical behaviour of microtubule length in the bounded and unbounded growth regimes is sketched in Figure 1.5.

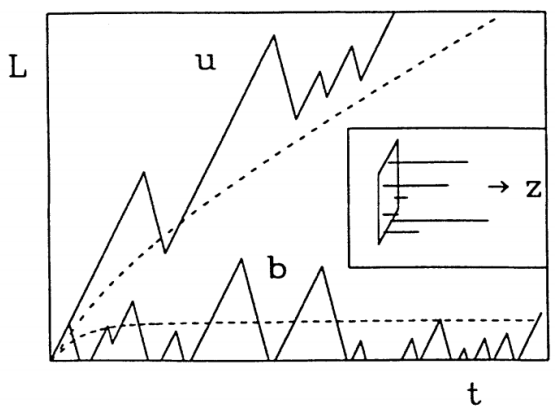

Figure 1.5: Typical behaviour of the length of microtubules undergoing dynamic instability in the $(\mathrm{u})$ unbounded and in the (b) bounded-growth regime as a function of time. Dashed lines represent the average value of the length over many microtubules. Image is taken from (Dogterom \& Leibler, Phys. Rev. Lett. 1993).

\section{The bounded-growth regime}

If $l^{-}>l^{+}$the system reaches the steady-state, i.e. the state in which all quantities of the model no longer depend on time. Solving Eqs. (1.1) and (1.2) when $\frac{\partial}{\partial t} m^{ \pm}(t, l)=$ 0 is then straightforward, and the solution is

$$
\begin{aligned}
& m^{+}(l)=\frac{r_{n}}{v^{+}} e^{-l / \bar{l}}, \\
& m^{-}(l)=\frac{r_{n}}{v^{-}} e^{-l / \bar{l}},
\end{aligned}
$$


where

$$
\bar{l}=\left(\frac{r_{c}}{v^{+}}-\frac{r_{r}}{v^{-}}\right)^{-1}>0 .
$$

The existence of a finite typical length for microtubules is strictly linked to the fact that their lifetime is finite as well. Indeed, it is possible to show (Bicout, Phys. Rev. E, 1997) that the average lifetime of a microtubule with initial length $l_{0}$ and initial state $\sigma$ is

$$
\tau\left(l_{0}, \sigma\right)=\frac{r_{c}+r_{r}}{v^{-} r_{c}-v^{+} r_{r}} l_{0}+\frac{v^{+}+v^{-}}{v^{-} r_{c}-v^{+} r_{r}} \delta_{\sigma,+},
$$

with full lifetime probability density function given by

$$
\begin{aligned}
& L_{+}\left(t \mid l_{0}\right)=\Theta\left(t-\frac{l_{0}}{v^{-}}\right) \frac{r_{c}}{v^{+} t+l_{0}} e^{-\frac{1}{v^{+}+v^{-}}\left[r_{r}\left(v^{+} t+l_{0}\right)+r_{c}\left(v^{-} t-l_{0}\right)\right]} \\
& \times\left[l_{0} I_{0}\left(\frac{2}{v^{+}+v^{-}} \sqrt{r_{c} r_{r}\left(v^{+} t+l_{0}\right)\left(v^{-} t-l_{0}\right)}\right)\right. \\
& \left.+\frac{v^{+}}{r_{c}} \sqrt{\frac{r_{c}}{r_{r}} \frac{v^{+} t+l_{0}}{v^{-} t-l_{0}}} I_{1}\left(\frac{2}{v^{+}+v^{-}} \sqrt{r_{c} r_{r}\left(v^{+} t+l_{0}\right)\left(v^{-} t-l_{0}\right)}\right)\right] \text {, } \\
& L_{-}\left(t \mid l_{0}\right)=\delta\left(t-\frac{l_{0}}{v^{-}}\right) e^{-r_{r} t} \\
& +\Theta\left(t-\frac{l_{0}}{v^{-}}\right) \frac{r_{c} r_{r} l_{0} e^{-\frac{1}{v^{+}+v^{-}}\left[r_{r}\left(v^{+} t+l_{0}\right)+r_{c}\left(v^{-} t-l_{0}\right)\right]}}{\sqrt{r_{c} r_{r}\left(v^{+} t+l_{0}\right)\left(v^{-} t-l_{0}\right)}} \\
& \times I_{1}\left(\frac{2}{v^{+}+v^{-}} \sqrt{r_{c} r_{r}\left(v^{+} t+l_{0}\right)\left(v^{-} t-l_{0}\right)}\right),
\end{aligned}
$$

where $I_{0}(\cdot)$ and $I_{1}(\cdot)$ are the modified Bessel functions of the first kind of order 0 and 1 , respectively.

\section{The unbounded-growth regime}

When $l^{+}>l^{-}$or, in other words, when the polymerization is faster then the depolymerization, the system is in the so-called unbounded-growth regime: $\bar{l}$ is no longer the typical length of microtubules, and the system does not reach the steady-state. Instead, the average length of microtubules grows linearly in time, as

$$
\langle l\rangle=V t=\frac{v^{+} r_{r}-v^{-} r_{c}}{r_{c}+r_{r}} t
$$


while the variance is

$$
\left\langle l^{2}\right\rangle-\langle l\rangle^{2}=\frac{r_{c} r_{r}}{\left(r_{c}+r_{r}\right)^{3}}\left(v^{+}+v^{-}\right)^{2} t .
$$

The length distribution asymptotically - for great $t$, approaches a Gaussian distribution. Eq. (1.9) implies that, in contrast with the bounded-growth case, here some microtubules grow indefinitely and have an infinite lifetime. However, due to their stochastic behaviour, it is possible that after undergoing a catastrophe, a microtubule does not undergo a subsequent rescue, but it completely depolymerizes, and thus it has a finite lifetime. More generally, there exists a finite fraction of microtubules that do not grow indefinitely, but they have a finite lifetime. We call this fraction ultimate survival probability and we denote it with $S_{\sigma}\left(l_{0}\right)$. It is possible to prove that

$$
\begin{gathered}
S_{+}\left(\infty \mid l_{0}\right)=1-\frac{v^{-} r_{c}}{v^{+} r_{r}} e^{\frac{l_{0}}{\bar{l}}}, \\
S_{-}\left(\infty \mid l_{0}\right)=1-e^{\frac{l_{0}}{\bar{l}}},
\end{gathered}
$$

and that the Eqs. (1.6), (1.7), and (1.8), holds for the unbounded growth-regime as well, except they need to be rescaled by $S_{\sigma}\left(l_{0}\right)$, as we are now looking at the lifetime of a microtubule conditional to the fact that its lifetime is finite (Bicout, Phys. Rev. E, 1997).

\subsection{Outline}

The outline of the thesis is the following. In Chapter 2, we present a computational stochastic model based on the Dogterom-Leibler model and aimed at explaining how the regulation of microtubule ends is important for the amplification of the number of longitudinal microtubules of the cortical array. We first introduce the experiments that motivate the research presented in part of this thesis. Then, we use our computational model to confirm the hypothesis proposed by the experimental observations: amplification occurs when both the microtubule minus end is stable, and when the probability that the newly-created plus end of a microtubule after a severing event due to the action of katanin suddenly enters the growing state is large enough.

In Chapter 3 we analytically study the computational model introduced in Chapter 2. While in Chapter 2 we studied the model for microtubules in the unbounded-growth regime, here we focus on the bounded-growth regime. We observe that, in order to have amplification, the probability of a newly-created plus end after severing to enter the growing state needs to be larger than a 
critical threshold, defined by the model parameters. We then show that we can calculate this threshold probability by making use of an approximation consisting in considering only a small part of the system to describe it as a whole.

Experiments show that once the number of longitudinal microtubules amplifies and the number of transverse falls, the new longitudinal array is maintained in the new orientation. In Chapter 4, we explore the possible asymmetries that can explain this maintenance effect. In order to do so, we propose an analytical model of dynamic microtubules split in two distinct populations that compete for the same finite amount of building material and interact with each other to sever microtubules of the opposite population. We introduce an asymmetry in the dynamic parameters of the two populations and we observe that such an asymmetry can explain the maintenance of the new longitudinal array.

In Chapter 5, we present a theoretical model of dynamic microtubules and diffusing actin in a confinement. The model is based on recent experiments that showed that microtubules and actin can interact through a class of proteins called cytolinkers and that such an interaction can change the dynamic properties of the former and can transport the latter. Given that this system has never been studied before, in this chapter we are interested in making predictions about the possible consequences of the actin-microtubule interaction rather then in understanding the underlying mechanism that makes them interact. We identify the length scales involved in the transport mechanism and their dependency on the dynamic parameters of the model, in order to make the transport more efficient.

After the conclusions, and taking the cue from the models presented in Chapters 2 and 5, we finally present in the appendix a stochastic ageing model for individuals born in an initial state and evolving in different states, with the possibility for them to switch from one state to another. We show that this model exhibits some intriguing mathematical properties. 

CHAPTER 2

\section{CLASP and SPR2 promote microtubule reorientation by acting as tip regulatory factors}

Microtubules are very dynamic polymers, and their dynamics is involved in many cellular processes such as reorganization of the cortical array, chromosome segregation, cell migration, and many others (Alberts, 2015). Experiments performed in the last two decades, showed that the dynamics is important not only at microtubule tips, but it can also be induced from events at microtubule lattice: in particular, it has been shown that severing processes at microtubule lattice play a crucial role in the regulation of cytoskeleton (Lindeboom et al., Science, 2013 Burk et al., Plant Cell, 2001 - McNally et al., J. Cell Biol., 2006).

The protein katanin, named after the Japanese sword katana, had been identified as a microtubule-stimulated ATPase protein that severs microtubules (McNally \& Vale, Cell, 1993), inducing dynamics starting from their lattice. Experimental observations had shown that katanin is a severing protein and it has three modes of operation. Firstly, katanin is responsible for the release of microtubule minus end from their nucleation site, enabling depolymerization and consequently treadmilling of the minus end (Wasteneys, J. Cell Science, 2002). The second way consists of the

The experiments reviewed in this chapter have been designed and carried out by Jelmer J. Lindeboom and David W. Ehrhardt from the Carnegie Institution for Science (United States of America), and Masayoshi Nakamura from Nagoya University (Japan). 
severing along the whole length of the microtubules at apparently random locations (Roll-Mecak and Vale, J. Cell Biol., 2006). Finally, katanin localizes at crossovers between differently oriented microtubules in the cortical microtubule array of etiolated $^{1}$ hypocotyl of Arabidopsis thaliana, and there it severs microtubules (Lindeboom et al., Science, 2013).

Intuitively, we can hypothesize that these last two severing mechanisms produce an increase in the number of microtubules, as well as they shorten their average length.

In this chapter we will focus on the third way for the katanin to sever microtubules and on the effect of this severing on the increase of their number. In section 2.1 we will investigate the role of katanin in the severing-driven reorientation of cortical microtubule array in dark grown cells of Arabidopsis thaliana, and we will introduce a theoretical model able to explain the underlying mechanisms behind such a reorientation. In section 2.2 and 2.3 we will present the experiments that determine two crucial regulatory factors for respectively plus and minus end that enable the reorientation, and with our model we will test our assumptions about how these two regulatory factors work. In the last section, we will analytically solve a model for microtubules undergoing dynamic instability at both plus and minus end, to test whether or not minus end instability is important for the steady-state microtubule length distribution when in absence of severing.

\subsection{The cortical microtubule array undergoes a katanin-mediated reorientation}

\section{Experimental observations}

The cortical microtubule array is an unique acentrosomal cytoskeletal structure in plant cells, and it is essential for the morphogenesis of the organism as it controls the direction of the anisotropic cell expansion. The function of the array depends on the spatial organization of its microtubules. It has been shown that microtubules change their dynamic behaviour - and hence their arrangement, as a response to both chemical and physical environmental signals (Dixit \& Cyr, Plant Cell, 2004).

A striking example of such a change in spatial organization occurs in etiolated hypocotyls of Arabidopsis Thaliana, and consists of the reorientation of the array from a transverse to a longitudinal direction to the growth direction of plant axis after the exposure to blue light. Particularly, one can observe that katanin

\footnotetext{
${ }^{1}$ The etiolation is a process that makes flowering plants undergo alterations when they grow in absence of light. Such alterations include, e.g., smaller leaves and a pale yellow color, due to the absence of chlorophyll.
} 


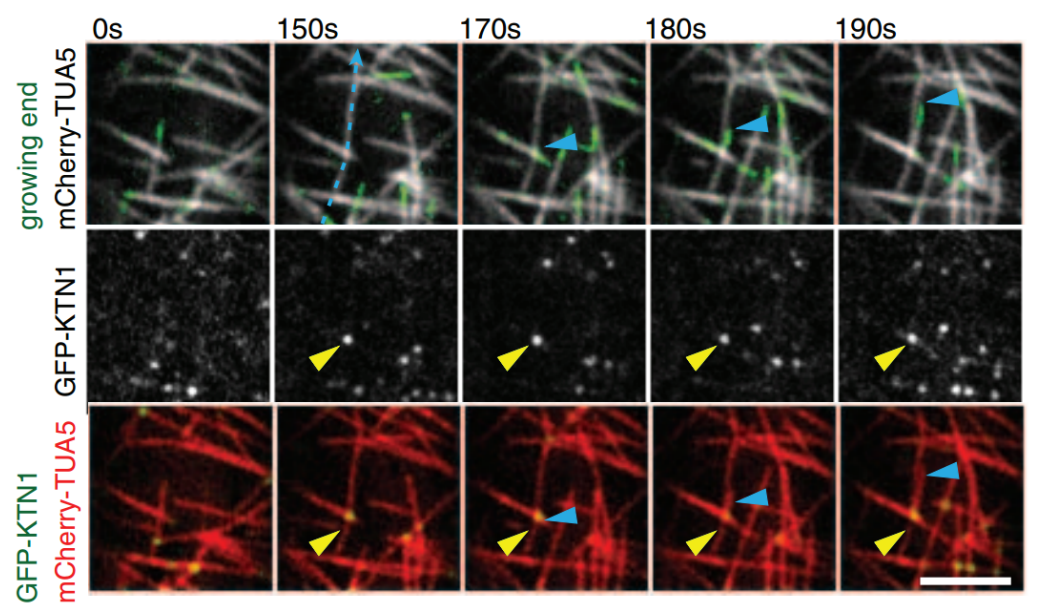

Figure 2.1: Katanin localization (yellow arrowheads) at a crossover (blue arrowheads). Image taken from (Lindeboom et al., Science, 2013).

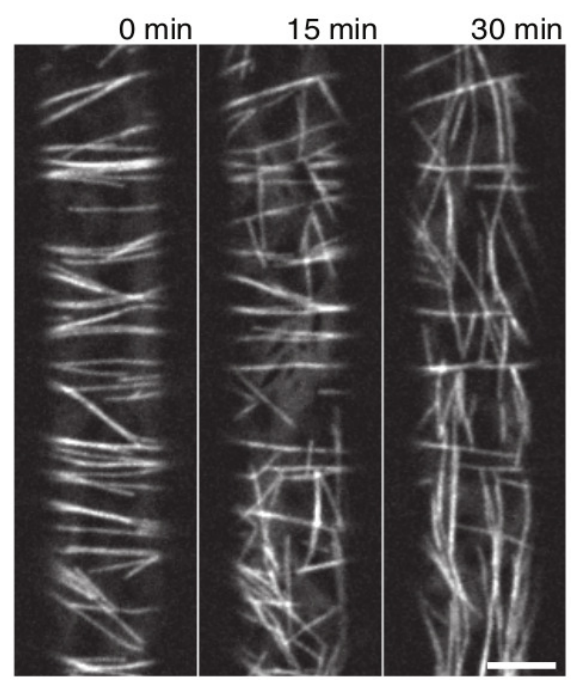

Figure 2.2: Reorientation of the cortical microtubule array of an Arabidopsis thaliana cell. Image taken from (Lindeboom et al., Science, 2013). 
A

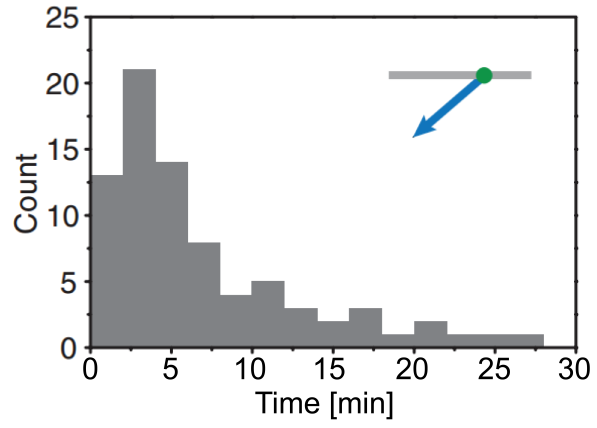

B

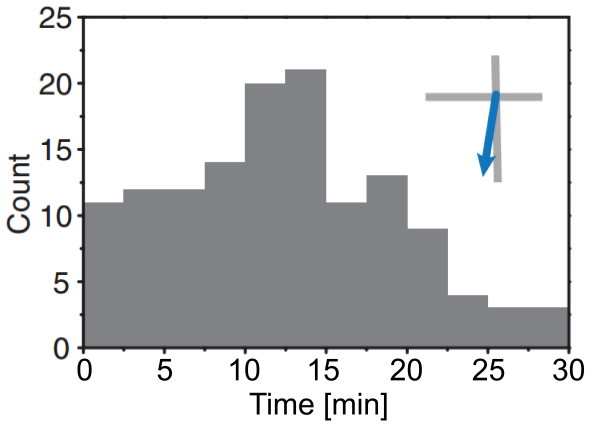

Figure 2.3: Count of microtubule creations as a function of time after blue light stimulation, (A) associated with a nucleation complex, (B) and associated with a crossover. Image taken from (Lindeboom et al., Science, 2013).

localizes at crossovers between differently oriented microtubules (see Figure 2.1) and there preferentially severs longitudinal, resulting in the effective creation of a new longitudinal microtubule. Usually, the newly-created plus end after severing undergoes a catastrophe due to the absence of a stabilizing GTP cap at the severing site. Here, however, due to a non-completely-understood downstream effect of photoactivation as a response to the blue light, a fraction of the newlycreated plus ends are stabilized and immediately grow. These newly-created lagging microtubules have then the opportunity to create new crossovers with the initial transverse microtubules, and hence they have the opportunity to undergo new severing events. This amplification mechanism, indeed, creates in around 30 minutes a new population of microtubules orthogonal to the initial transverse array, see Figure 2.2. Recent experiments have shown that in this system around $83 \%$ of microtubule creations are katanin-mediated (Lindeboom et al., Science 2013). After the stimulation with blue light, in fact, we can observe a decreased rate of microtubule nucleation in advantage of an increased rate of severing mediated microtubule creations at crossovers, see Figure 2.3. It is however important to underline that the creation of a crossover does not necessarily imply the occurrence of a severing event. Indeed a severing event at a crossover is not an instantaneous event that happens just after the crossover creation, but it requires a certain waiting time, due to the finite time needed by katanin to recruit at the severing site. Because of this, any crossover might be removed by the depolymerization of either of the two microtubules involved, preventing the recruitment of katanin on the lattice of the microtubule, and hence the severing event.

This reorientation is not a proper reorientation, in the sense that individual mi- 


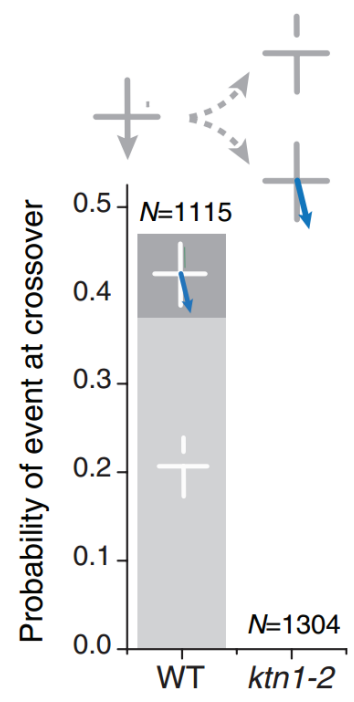

Figure 2.4: Probability of severing event at crossovers in Arabidopsis thaliana WT and ktn1-2. In the WT case, a fraction of the newly-created plus end after severing is stabilized and immediately grows. Image taken from (Lindeboom et al., Science, 2013).
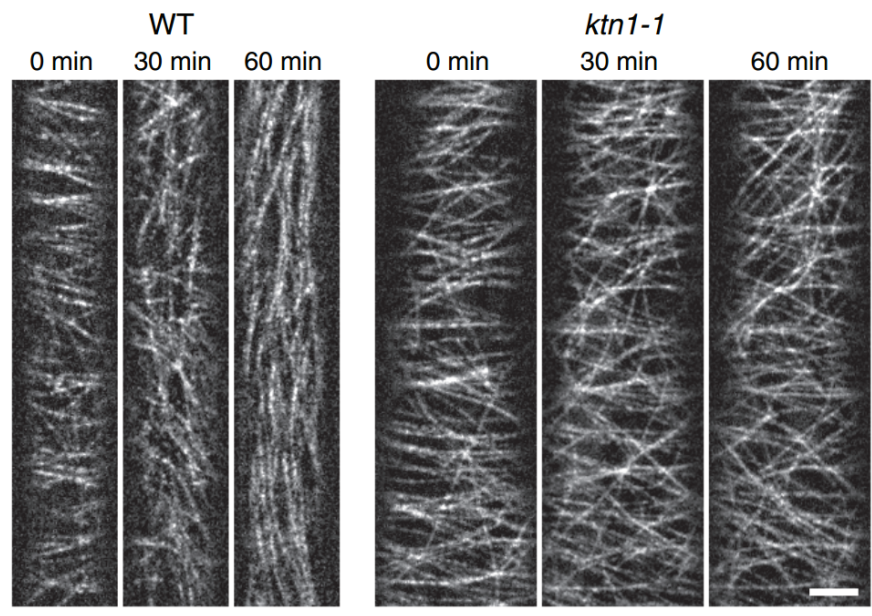

Figure 2.5: Reorientation of the cortical microtubule array in WT and ktn1-1. While the former exhibits complete reorientation after 60 minutes time, the latter exhibits a neglectable degree of reorientation. Image taken from (Lindeboom et al., Science, 2013). 
crotubules do not change their orientation. Instead, it consists of the disappearance of a population of microtubules - the transverse one, due their finite lifetime, in advantage of the creation of a second population - the longitudinal. As we will see in Chapter 4, the disappearance of transverse microtubules provides the building material for the creation of the second.

As a final proof that indeed katanin-mediated severing events drive the reorientation process, experiments performed with Arabidopsis katanin suppressed mutants ktn1-1 and ktn1-2 do not exhibit severing events at microtubule crossovers (see Figure 2.4), and hence do not exhibit any reorganization of the cortical microtubule array (Zhang et al., Current Biology 2013), see Figure 2.5.

\section{The model}

To describe the impact of katanin mediated severing events on the initial amplification of the number of longitudinal microtubules, we set up a stochastic model of longitudinal microtubules undergoing dynamic instability in a background of stable transverse microtubules, see Figure 2.6 (Nakamura et al., J. Cell Biol. 2018). In order to be able to consider the initial transverse array as a constant background, we focus on the initial stage of the process, i.e. up to 500 seconds after the exposure to blue light (see Figure 2.7). After that time, the experiments reveal the start of

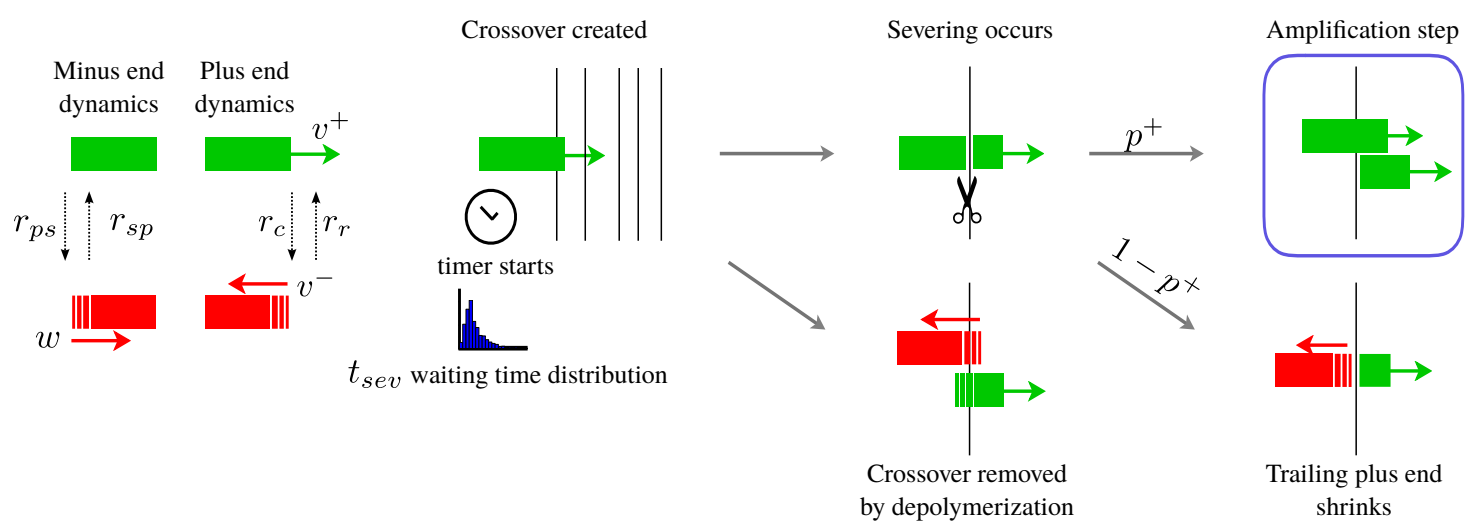

Figure 2.6: Schematic of the model. The competition between severing at a crossover and crossover removal due to depolymerization of either of the two microtubule ends plays a crucial role in the amplification process, as well as the fraction $p^{+}$of rescue after severing.

the suppression of the initial array. Hence, after 500 seconds, the dynamics of the transverse microtubules can no longer be ignored. 


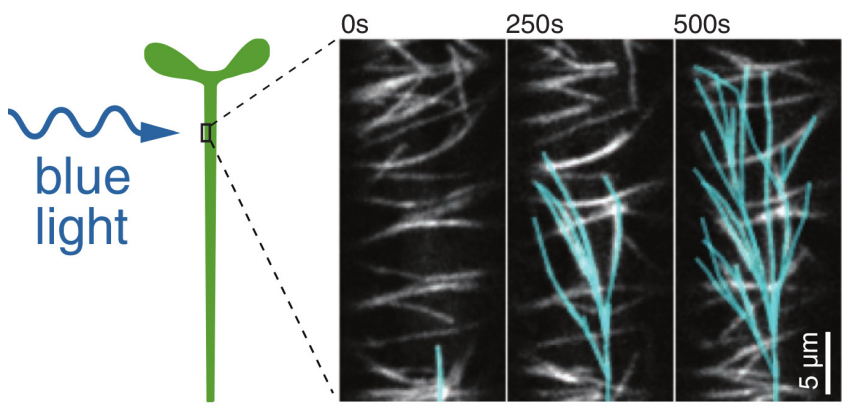

Figure 2.7: Amplification of the number of longitudinal microtubules in the first 500 seconds after the exposure to blue light. Image taken from (Lindeboom et al., Science, 2013).

The model keeps track of the time evolution of a single longitudinal microtubule and all of its severing-created descendants. Dynamic parameters for longitudinal microtubules are the growing and shrinking speed for the plus end, $v^{+}$and $v^{-}$ respectively, shrinking speed for the minus end $w$, catastrophe rate $r_{c}$ and rescue rate $r_{r}$ for the plus end, rate of passing from paused state to shrinking state $r_{p s}$, and rate of passing from the shrinking state to the paused state $r_{s p}$ for the minus end.

The experimentally measured distribution of the spacing $\Delta x$ between initial transverse microtubules can be reasonably fit by the distribution function

$$
f(\Delta x)= \begin{cases}0 & \text { if } \Delta x<0.5 \mu \mathrm{m} \\ \frac{1}{d} e^{-\frac{\Delta x}{d}} & \text { if } \Delta x \geq 0.5 \mu \mathrm{m} .\end{cases}
$$

The spacing scale parameter $d$ is chosen such that the mean spacing $d_{a v g}$ between neighbour in the initial transverse array is consistent with the experimentally measured one, see Tables 2.1 and 2.2, and Figures 2.8 and 2.14.

Every time a longitudinal microtubule plus end impinges on a transverse microtubule, it creates a crossover. This crossover is either removed by the shrinkage of either of ends of the microtubule, or it leads to a severing event if it survives long enough. Whether the severing event occurs or not is determined by a competition between the waiting time distribution for severing events at a crossover, and the survival time distribution of crossovers from microtubule depolymerization. This competition will be studied in details in Chapter 3. While the survival time of crossovers from depolymerization depends mainly on the dynamic parameters of microtubules, the severing waiting time is an intrinsic property of the crossovers. 
The choice of a good severing waiting time distribution needs to fulfill two different requirements: it is the function that best fits the experimentally measured data (Nakamura et al., J. Cell Biol., 2018 - Lindeboom et al., J. Cell Biol., 2018), and it best represents the recruitment of katanin at microtubule crossovers. Using, e.g., an exponential function for the waiting time distribution would not work, since every crossover needs a certain amount of time to recruit enough katanin for the severing event to occur, and an exponential function bears a high probability of severing at short times. Instead, a peaked distribution expresses better the idea that katanin requires some time to accumulate at crossovers. Therefore, a reasonable choice for the severing waiting time distribution is the Gamma probability density function (Papoulis, 1984), i.e.

$$
W_{k, \theta}(t)=\frac{t^{k-1}}{\theta^{k} \Gamma(k)} e^{-\frac{t}{\theta}},
$$

where $k$ and $\theta$ are the shape and scale parameters of the distribution, and

$$
\Gamma(k)=\int_{0}^{+\infty} d x x^{k-1} e^{-x}
$$

is the Euler gamma function (Abramowitz \& Stegun, 1965).

If a severing event at a crossover happens, the newly-created plus end of the lagging microtubules either is stabilized and suddenly enters the growing state with probability $p^{+}$, or the shrinking state with probability $1-p^{+}$. Similarly, the newly-created minus end of the leading microtubule either shrinks with probability $q^{-}$, or pauses with probability $1-q^{-}$.

In the following two sections, we are going to use our model to quantify the magnitude of the longitudinal amplification in the case of changed stability at either of the two microtubule ends.

\subsection{CLASP stabilizes newly-created plus ends after severing}

\section{Overview of the experiments}

Several proteins that track the plus end of Arabidopsis thaliana growing microtubules have been identified (Bisgrove et al., Plant Physiol., 2004). Here, we focus on the effect of End Binding Protein 1 (EB1), Cytoplasmic-Linker-Associated Protein (CLASP) and SPIRAL1 (SPR1) on the probability of having a rescue after severing at the microtubule plus end, and hence on the speed of the amplification of the number of longitudinal microtubules. To do that, we compare the different 
outcomes in the reorientation process in the microtubules that lack those proteins. We refer to those microtubules as mutants, and we denote them as $3 x-e b 1^{2}$, clasp, and spr1, and we compare the results with the control reorientation outcome of the Wild Type (WT) (Lindeboom et al., J. Cell Biol., 2018).
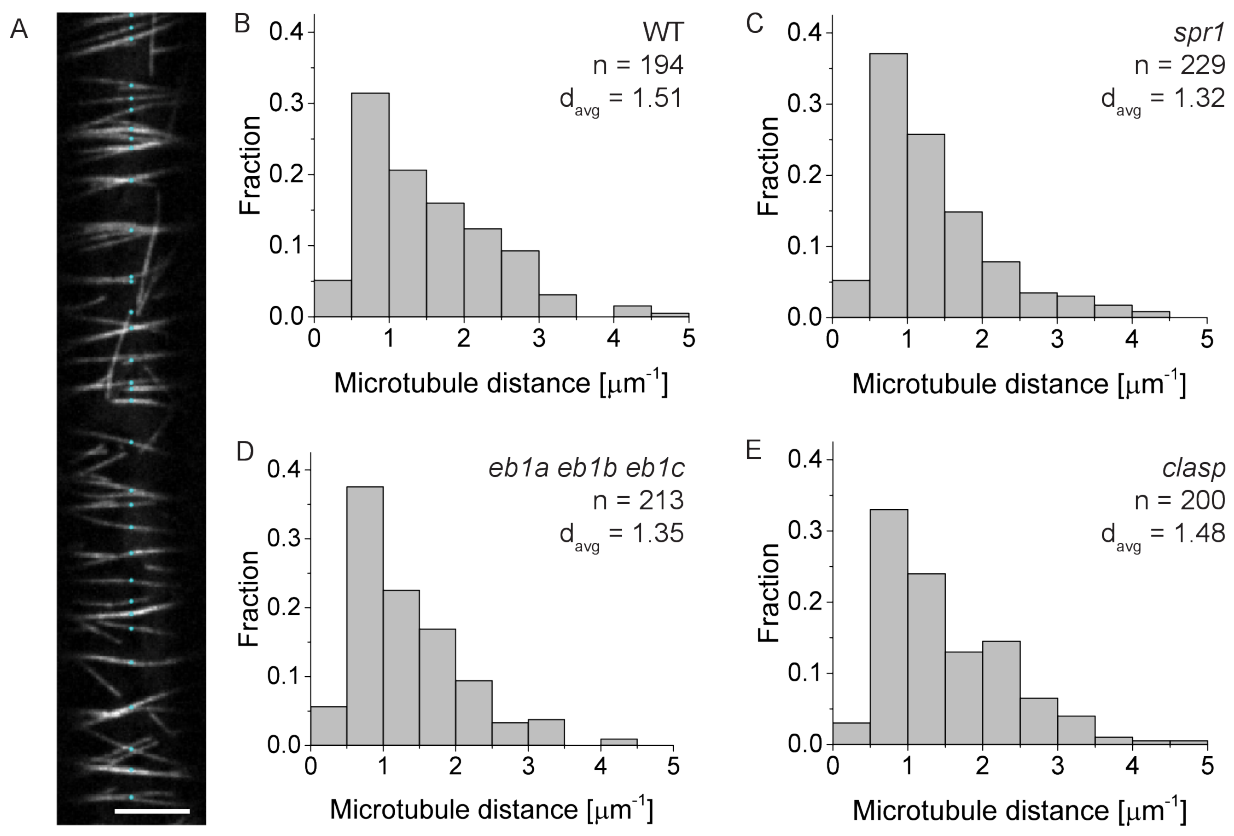

Figure 2.8: Transverse microtubules bundle before the exposure to blue light. Image taken from (Lindeboom et al., J. Cell Biol., 2018).

In vivo quantitative imaging shows that the reorientation process starts after the exposure to blue light, and in around 15 minutes the reorientation of WT and spr1 is complete. After 30 minutes from the first exposure to blue light, also the cortical microtubule array of $3 x-e b 1$ mutant is completely reoriented, while in clasp mutant, even though one can observe an increase in the number of longitudinal microtubules, the reorientation does not happen, see Figure 2.9. Therefore, we explore all possible reasons why the cortical microtubule array of clasp mutant behaves differently from WT and other mutants.

Experimental measurements show that WT and the three mutants exhibit different dynamic behaviours, see Table 2.1: indeed, clasp and spr1 have a greater plus end growth speed than WT, whilst $3 x-e b 1$ and WT show a comparable growth speed. The shrinkage speed of WT is greater, equal, and smaller than

\footnotetext{
${ }^{2}$ The $3 x$ - comes from the fact that in Arabidopsis thaliana, EB1 is encoded in three genes: $a$, $b$, and $c$. They are all disrupted to create $3 x-e b 1$.
} 


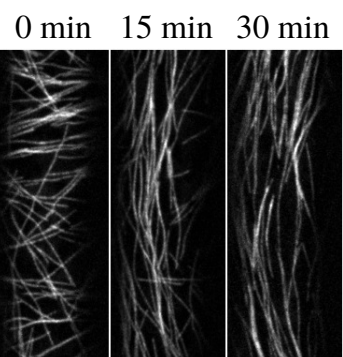

WT

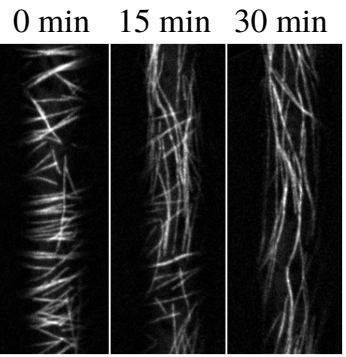

sprl

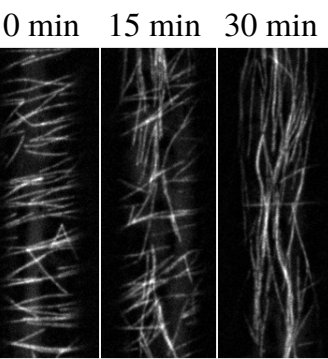

$3 x-e b 1$

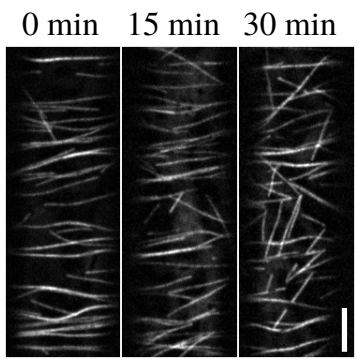

clasp

Figure 2.9: Reorientation of the cortical array in WT, spr1, 3x-eb1, and clasp. Image taken from (Lindeboom et al., J. Cell Biol., 2018).

spr1, clasp, and 3x-eb1 respectively. As regards dynamic instability rates, we can observe a reduced catastrophe rate in the spr 1 mutant, while clasp and $3 x$-eb1 show catastrophe rates comparable to WT. Finally, one can observe a very low rescue rate in clasp mutant, compared to the WT, in agreement with the wellstudied observation that CLASP protein is a potent microtubule rescue promoter (Al-Bassam et al., Dev. Cell, 2010). Looking at the dynamic parameters, one can notice that all genotypes are in the unbounded-growth regime. Indeed, the average speed of microtubules are $V_{W T}=0.043 \mu \mathrm{m} \mathrm{s}^{-1}, V_{3 x-e b 1}=0.034 \mu \mathrm{m} \mathrm{s}^{-1}$, $V_{\text {clasp }}=0.032 \mu \mathrm{m} \mathrm{s}^{-1}$, and $V_{\text {spr } 1}=0.061 \mu \mathrm{m} \mathrm{s}^{-1}$ where $V=\frac{v^{+} r_{r}-v^{-} r_{c}}{r_{r}+r_{c}}$ has been defined in Eq. (1.9). Therefore, one can observe some degree of difference in the dynamics of different mutants.

These measurements of the probability of rescue after severing highlight two interesting facts. First of all, one can observe a small but relevant difference in such a probability depending on the orientation of the microtubule. Indeed, all genotypes show a greater probability of rescue after severing for the longitudinal microtubules than the transverse. The second observation is that such a probability is very low - around 3\% in clasp mutant, suggesting that CLASP protein acts as a protector against depolymerization after a severing event, see Table 2.1.

Hence, from these experimental observations, we formulate the hypothesis that the reason why clasp mutant fails in the amplification mechanism is because of either the low probability of rescue after severing or the low intrinsic rescue rate.

A final observation concerns the severing waiting time at crossovers. As we introduced in the previous section, the severing waiting time distribution for all mutants is nicely fitted by a Gamma probability density function. However, the different mean severing waiting times experimentally measured, and the different widths of the distributions for clasp and 3x-eb1 with respect to WT (Figure 2.10), suggest that CLASP antagonizes the activity of katanin, whilst EB1 promotes it, 
see Table 2.1. Experimentally, one can only measure the severing waiting time distribution conditional that the severing had occurred and the crossover had not been removed by dynamic instability of the microtubule. Then we have chosen scale and shape parameters for the severing waiting time distributions such that the output distributions optimally fit the experimental ones, see Figure 2.10.
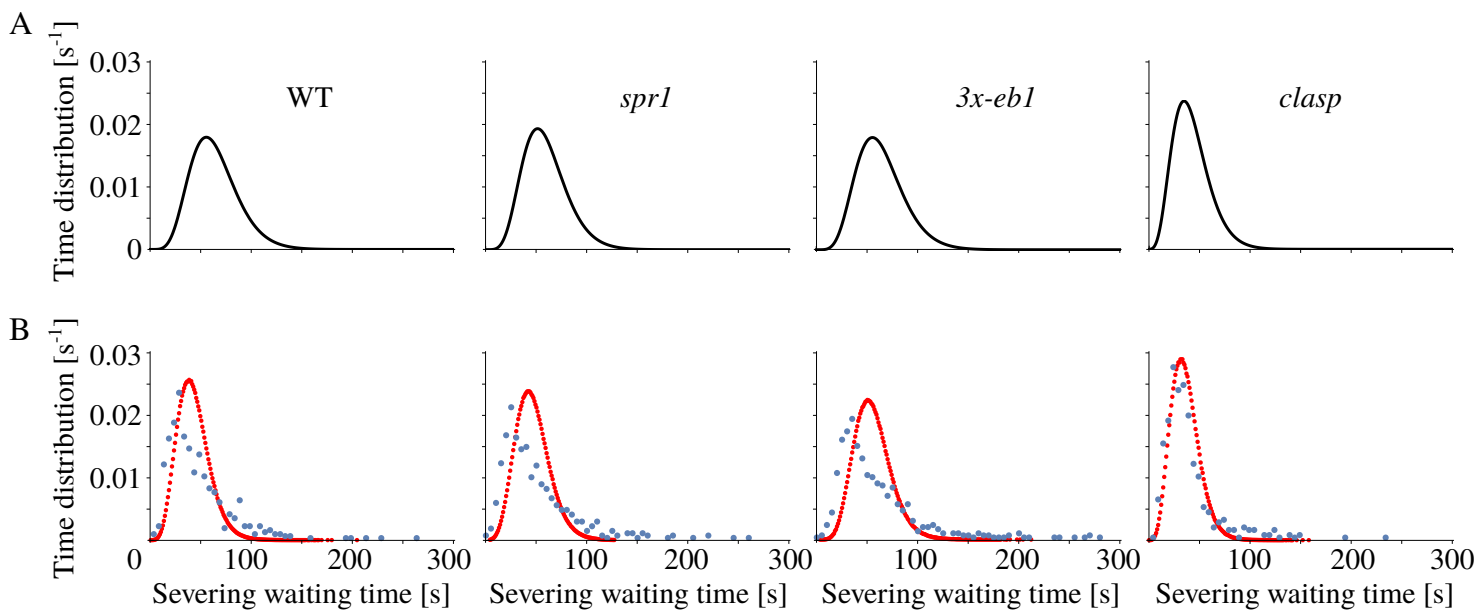

Figure 2.10: (A) Intrinsic severing waiting time distribution used in the simulations, and (B) comparison between experimentally observed (blue dots) and computed conditional severing waiting time distribution (red dots) for WT, spr1, 3x-eb1, and clasp. Image taken from (Lindeboom et al., J. Cell Biol., 2018).

\section{Computational approach}

To assess whether a high probability of rescue after severing plays a more important role than a low intrinsic rescue rate, we make use of the computational model introduced in the previous section. As the experiments only concern the behaviour of the plus end of microtubules, and the minus end does not exhibit a very fast dynamics, in our model we keep plus ends dynamic and we hold minus ends stable. In other words, for all mutants we have $w=0, r_{p s}=0$, and $q^{-}=0$.

Since we are only interested in the amplification mechanism for the number of longitudinal microtubules in the first 500 seconds of the whole reorientation process, we allow severing only for longitudinal microtubules. Every simulation follows the fate of one longitudinal microtubule and all of its descendants in the background of transverse microtubules. After 500 simulated seconds, either the simulation output is exctinction, i.e. the initial microtubule and all of its descendants have shrunk to length $l=0$ because of dynamic instability, or it is amplification, i.e. 
the initial microtubule has a surviving offspring due to severing at crossovers. For every genotype we run $N=5 \times 10^{4}$ trials of the same simulation, and we average the results over $N$.

For all genotypes, simulations result in an average amplification, even though every single trial exhibits a finite probability of extinction, see Figure 2.11. This result is consistent with the experimental outcomes, since all genotypes observed even clasp mutant, show some degree or reorientation, see Figure 2.9. The result that every trial has a finite probability to lead to amplification depends on the fact that all genotypes are in the unbounded-growth regime. Hence some microtubules live indefinitely (Bicout, Phys. Rev. E, 1997) and they and/or their descendants are severed an exponentially increasing amount of times. The consequence of this is that the average number of microtubules over all simulations shows amplification. Even if the amplification occurs for all genotypes, we observe a different speed of amplification depending on the mutant. As expected, clasp and $3 x-e b 1$ exhibit a very slow speed of amplification compared to those of the WT and spr1. In particular, amplification of clasp is almost non-noticeable after the first 500 seconds, see Figure 2.11B.
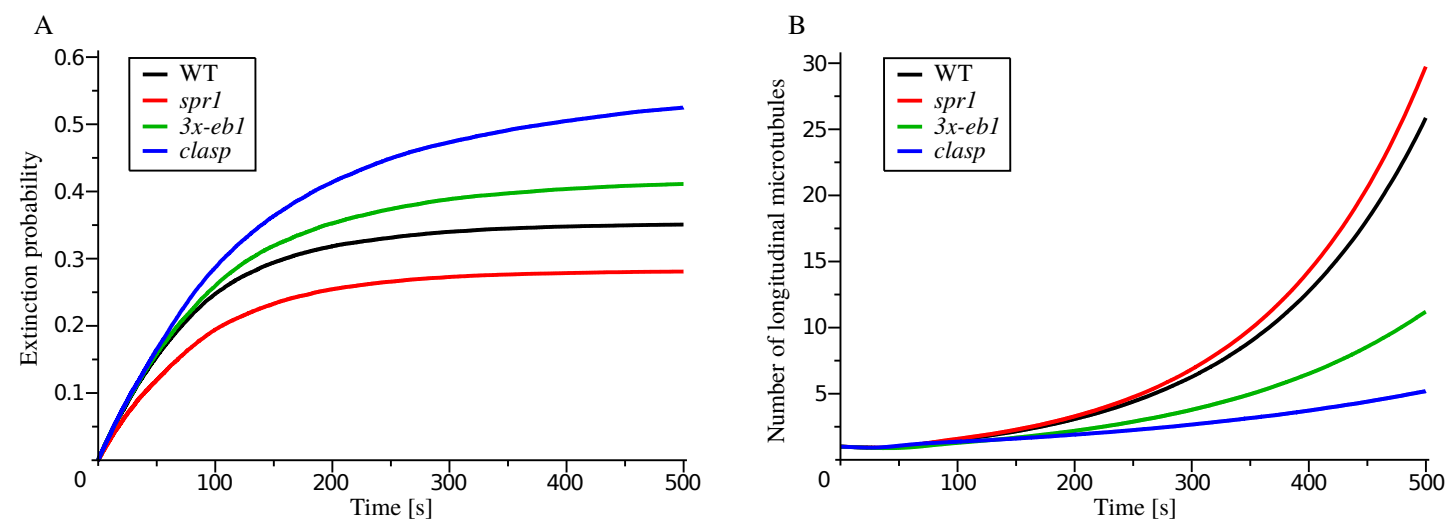

Figure 2.11: Time evolution of (A) the extinction probability, and (B) the number of longitudinal microtubules created by severing for WT, spr1, 3x-eb1, and clasp. Results are averaged over $N=5 \times 10^{4}$ trials of the same simulation. Image taken from (Lindeboom et al., J. Cell Biol., 2018).

If we look at the parameters used for WT and clasp, we notice that the severing waiting time in clasp mutant is on average shorter than in WT, as well as clasp growth speed is higher than WT one. This feature, together with the fact that shrinkage speed and catastrophe rate of the two genotypes are similar, would suggest a higher speed of amplification for clasp rather than for the WT. However, both experiments and simulations show the opposite behaviour. Therefore, the 
reason must be explained by the other two different parameters that these two genotypes exhibit: probability of rescue after severing $p^{+}$, or intrinsic rescue rate $r_{r}$. In order to understand which of these two parameters plays a more important role for the different behaviour of microtubules, we create two synthetic in silico mutants: WT microtubules with clasp intrinsic rescue rate $\left(\mathrm{WT} /\right.$ clasp $\left.r_{r}\right)$, and WT microtubules with clasp probability of rescue after severing (WT/clasp- $\left.p^{+}\right)$. Despite a very similar behaviour for the extinction probability of the two synthetic mutants, we observe a faster amplification speed for $\mathrm{WT} /$ clasp $r_{r}$, while $\mathrm{WT} /$ clasp$p^{+}$almost reproduces the amplification behaviour of the clasp mutant, see Figure 2.12. This result supports our hypothesis that the crucial role for the amplification of the number of longitudinal microtubules is played by the probability of rescue after severing $p^{+}$.
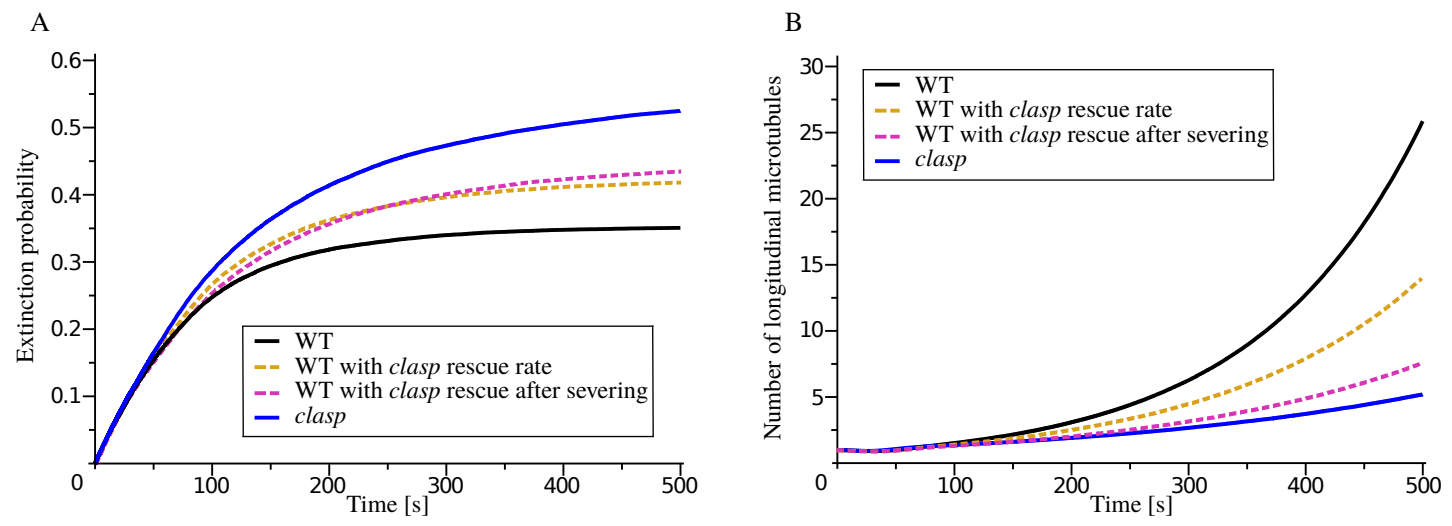

Figure 2.12: Time evolution of (A) the extinction probability, and (B) the number of longitudinal microtubules created by severing for WT and clasp, and for the synthetic mutants $\mathrm{WT} /$ clasp- $r_{r}$ and $\mathrm{WT} /$ clasp- $p^{+}$. Results are averaged over $N=5 \times 10^{4}$ trials of the same simulation. Image taken from (Lindeboom et al., J. Cell Biol., 2018).

To better quantify the importance of $p^{+}$rather than $r_{r}$ for the amplification mechanism, we perform a sensitivity analysis. We keep WT values as background and we change in turn $p^{+}$and $r_{r}$. As we are dealing with two unrelated parameters, we need to find a method to compare a change in the first with a change in the second. Since our interest is in the behaviour of microtubules in the unbounded-growth regime, we observe that the minimum value for $r_{r}$ such that a microtubule with WT background parameters is still in the unbounded-growth regime is $r_{r}=0.013$ 
events/second. Indeed, with such a rescue rate, we have

$$
V=\frac{v^{+} r_{r}-v^{-} r_{c}}{r_{r}+r_{c}}=0
$$

We compare the number of microtubules after 500 seconds with that rescue rate with the same quantity obtained with $W T$ background but $p^{+}=0$. Then, we tune $p^{+}$from 0 to 0.25 , and for every value of $p^{+}$we obtain the correspondent value for $r_{r}$ through the relation

$$
\frac{p_{W T}^{+}=0.15-0}{p^{+}}=\frac{r_{r, W T}=(0.026-0.013) \text { events } / s}{r_{r}} .
$$

Figure 2.13 shows that the amplification is more sensitive to the change of $p^{+}$rather

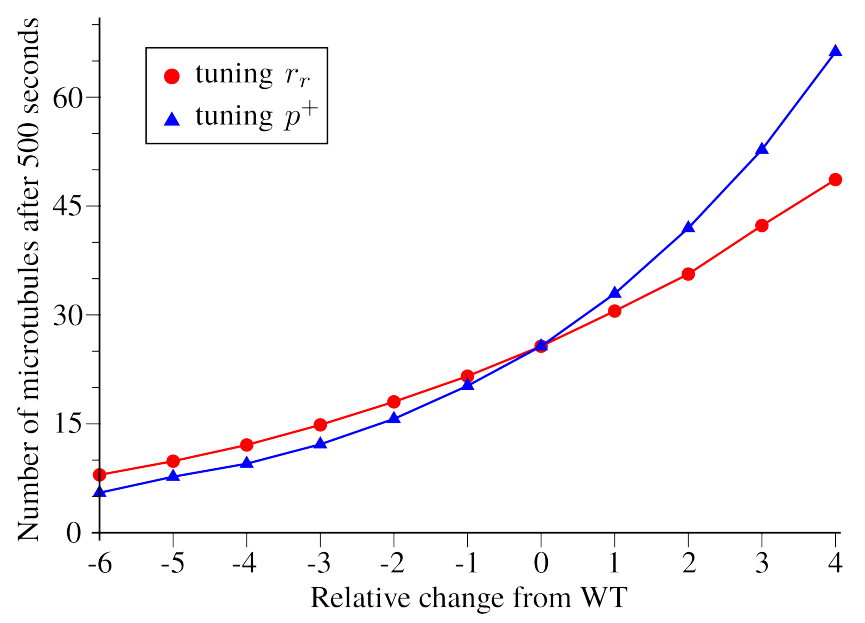

Figure 2.13: Sensitivity analysis of microtubules amplification to the change of $r_{r}$ and $p^{+}$. We counted the average number of microtubule after 500 seconds when we change $r_{r}$ (red dots), and $p^{+}$(blue triangles). From left to right, rescue rates $r_{r}$ are: $r_{r}=0.013$ events $/ s, r_{r}=0.015$ events $/ s, r_{r}=0.017$ events $/ s, r_{r}=0.019$ events $/ s$, $r_{r}=0.022$ events $/ s, r_{r}=0.024$ events $/ s, r_{r}=0.026$ events $/ s, r_{r}=0.028$ events $/ s$, $r_{r}=0.031$ events $/ s, r_{r}=0.033$ events $/ s$, and $r_{r}=0.035$ events $/ s$. Probability of rescue after severing $p^{+}$are, respectively: $p^{+}=0, p^{+}=0.025, p^{+}=0.05$, $p^{+}=0.075, p^{+}=0.1, p^{+}=0.125, p^{+}=0.15, p^{+}=0.175, p^{+}=0.2, p^{+}=0.225$, and $p^{+}=0.25$. Image taken from (Lindeboom et al., J. Cell Biol., 2018).

than $r_{r}$. This is a further evidence that, even though the intrinsic rescue rate and hence the dynamic instability of a microtubule is important for the amplification mechanism, the key parameter for the speed of amplification is the probability of rescue after severing. 
CLASP and SPR2 promote microtubule reorientation by acting as tip regulatory factors

\begin{tabular}{|ccccccc|}
\hline Parameter & Description & WT & $3 x-e b 1$ & clasp & spr1 & Units \\
\hline$v^{+}$ & Growth speed & 0.103 & 0.102 & 0.117 & 0.11 & $\mu \mathrm{m} \mathrm{s}^{-1}$ \\
$v^{-}$ & Shrinkage speed & 0.225 & 0.248 & 0.225 & 0.207 & $\mu \mathrm{m} \mathrm{s}^{-1}$ \\
$r_{c}$ & Catastrophe rate & 0.0058 & 0.0057 & 0.0062 & 0.0048 & $\mathrm{~s}^{-1}$ \\
$r_{r}$ & Rescue rate & 0.026 & 0.0237 & 0.0187 & 0.0261 & $\mathrm{~s}^{-1}$ \\
$p^{+}$ & Rescue after severing $(\|)$ & 0.15 & 0.13 & 0.03 & 0.1 & - \\
$p_{\perp}^{+}$ & Rescue after severing $(\perp)$ & 0.12 & 0.11 & 0.02 & 0.09 & - \\
$d$ & Spacing scale parameter & 1.01 & 0.85 & 0.98 & 0.82 & $\mu \mathrm{m}$ \\
$d_{\text {avg }}$ & Average bundle spacing & 1.51 & 1.35 & 1.48 & 1.32 & $\mu \mathrm{m}$ \\
$k$ & Gamma shape parameter & 7 & 8 & 6 & 8 & - \\
$\theta$ & Gamma scale parameter & 7.5 & 8.3 & 7.4 & 7.7 & $\mathrm{~s}$ \\
$t_{\text {sev }}$ & Mean severing waiting time & 47.9 & 59.8 & 39.6 & 52.7 & $\mathrm{~s}$ \\
\hline
\end{tabular}

Table 2.1: Plus end dynamics and crossover parameters.

\subsection{SPR2 protects minus end from fast depolymerization}

\section{Overview of the experiments}

In the previous section we have seen how the stability of newly-created plus ends after severing is important for the reorientation of the cortical microtubule array. The importance of the dynamics of the plus end is relatively well-understood. However how the dynamics of the minus end influences such a reorientation has not yet been investigated. Furthermore, how minus ends are controlled in higher plants is also currently unknown. The SPIRAL2 (SPR2) protein has recently been identified as a minus end tracker and a protector against subunit loss (Nakamura et al., J. Cell Biol., 2018). In vivo experiments in dark-grown etiolated hypocotyl of Arabidopsis thaliana show that SPR2 accumulates at newly-created minus ends after severing and stabilizes them, protecting them from a fast depolymerization. Our hypothesis is that without a stable minus end, i.e. in presence of fast minus end depolymerization, crossovers between differently oriented microtubules are more likely to be removed by the shrinkage of either of the two microtubules, as the effect of the minus end depolymerization is added to the one due to the dynamic instability of the plus end. Therefore, as a consequence of this increased crossover removal, katanin lacks recruitment spots on the microtubules, decreasing the number of severing events and then the effectiveness of the reorientation.

Interestingly, from the experiments there are no observations of polymerization at microtubule minus ends, but minus end dynamic instability only consists of periods of shrinkage and periods of pause (Shaw et al., Science, 2003).

To test the hypothesis that SPR2 promotes cortical microtubule array reorienta- 
tion by increasing the lifetime of potential severing site, we compare the dynamics and the efficiency of the reorientation of WT with those of the SPR2 lacking mutant, the spr2-2. The two different genotypes exhibit different dynamic behaviours at both plus and minus end, see Table 2.2. As regards the plus end, we observe an identical growth speed between WT and spr2-2, whilst the shrinkage speed of spr2-2 is higher than in the control case. However, spr2-2 exhibits a very low catastrophe rate, with a comparable - still higher, rescue rate than in WT case. This reduced catastrophe rate in spr2-2 mutant, suggests that SPR2 protein may have a destabilizing effect for microtubule plus end. The minus end, instead, shows a depolymerization for spr2-2 mutant almost three times faster than for WT. Moreover, spr2-2 has a very low shrink-to-pause rate and a very high pause-to-shrink rate, while WT exhibits exactly the opposite behaviour. This suggests that the minus end of spr2-2 mutant is almost always shrinking and the minus end of WT is mainly pausing. This hypothesis is confirmed by the experimental measurements, that show that in $91 \%$ of the observation time the spr2-2 minus end is shrinking, against $35 \%$ of the time for the WT. The effect of this further dynamic instability at the minus end for the length distribution of microtubules will be discussed in detail in the next section.

As regards the efficiency of the reorientation in response to the exposure to blue light, the experiments show that spr2-2 does not quickly reorient as in the case of WT, see Figure 2.15. Nevertheless also spr2-2 exhibits some very slow degree of reorientation. This observation is not enough to support our hypothesis that the lack of reorientation is due to the removal of crossovers consequent to the fast minus end depolymerization: indeed, it is still possible that SPR2 promotes katanin efficiency at crossovers. To test whether this is the case or not, the severing waiting time distribution from the moment of the creation of a crossover and the moment in which the severing event occurs has been measured, given that the crossover is not removed by dynamic instability. Intriguingly, one can observe no substantial difference between the two severing time distributions as an evidence that SPR2 neither stimulates nor represses katanin activity at microtubule crossovers. Furthermore, as we will see later in this section, with our simulations we can nicely fit both experimentally measured distributions with the same intrinsic severing waiting time distribution, see Figure 2.16.

A good method to test whether or not is the minus end depolymerization that prevents the reorientation from happening, is to count how many times the creation of a crossover leads to a severing event, and how many times a crossover is removed by depolymerization of either of the two ends. As we can see in Table 2.3, in WT the fraction of crossovers resolved by severing is greater than in spr2-2. Furthermore, looking at crossover removals by depolymerization, we can notice that in the spr2-2 mutant $84 \%$ of the cases the removal is due to minus end loss, 

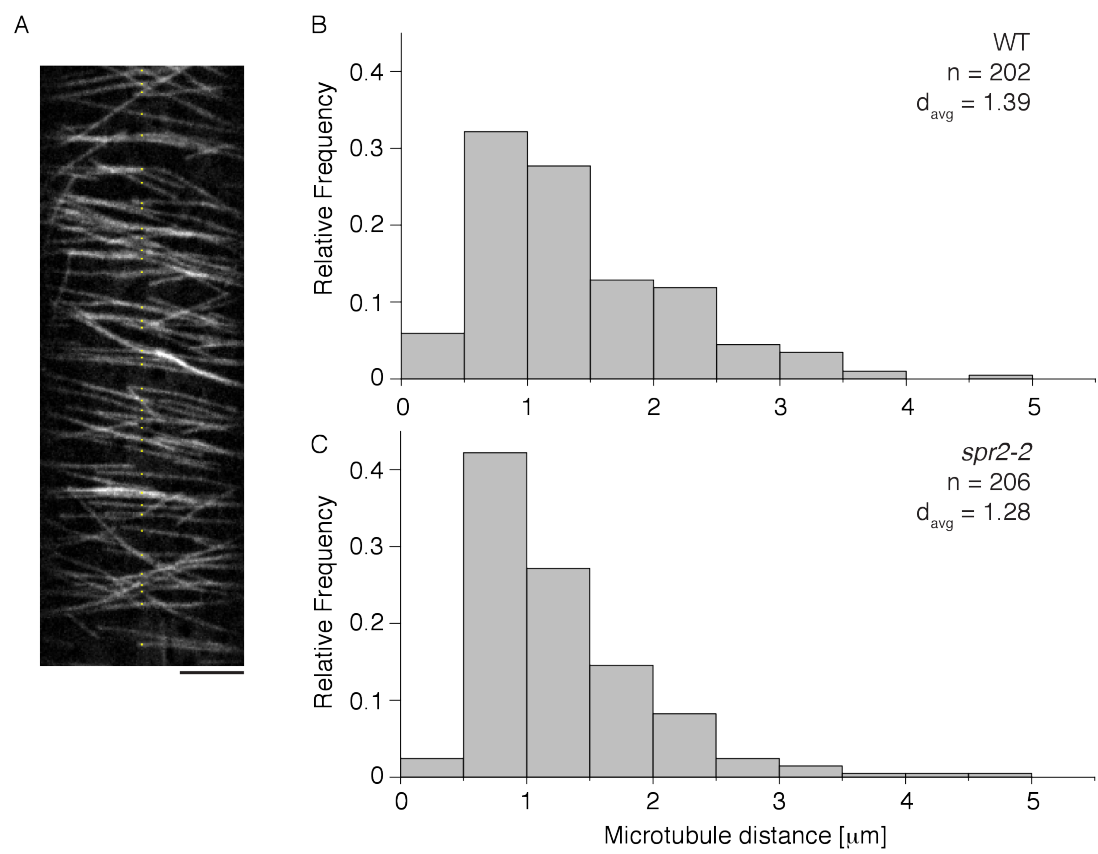

Figure 2.14: Transverse microtubule bundle before the exposure to blue light. Image taken from (Nakamura et al., J. Cell Biol., 2018).
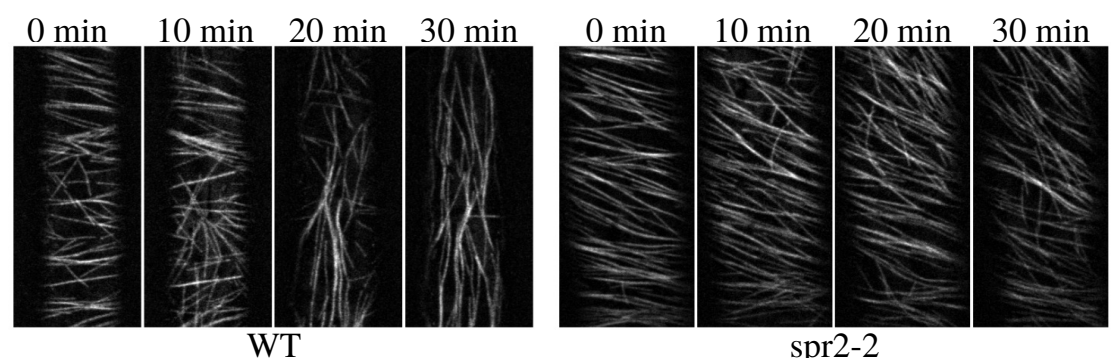

Figure 2.15: Reorientation of the cortical array in WT and spr2-2. Image taken from (Nakamura et al., J. Cell Biol., 2018). 

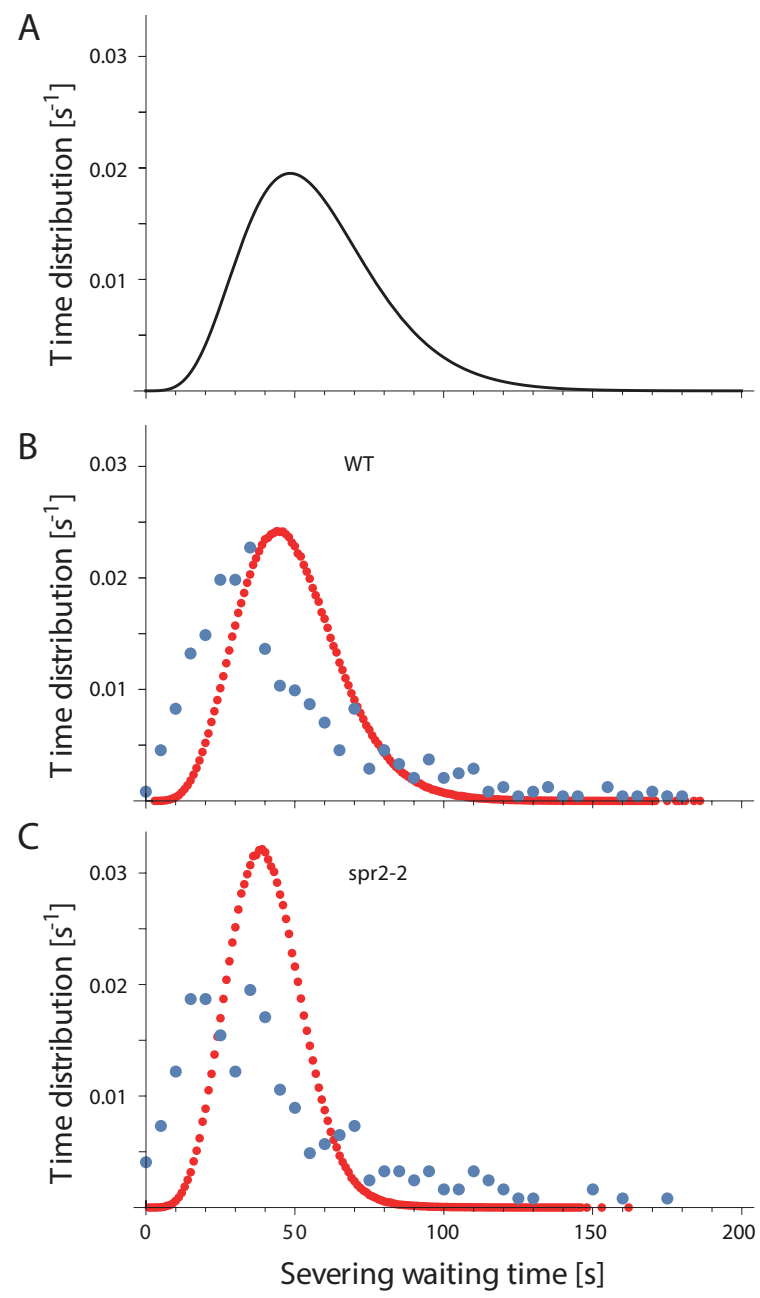

Figure 2.16: (A) Intrinsic severing waiting time distribution used in the simulations, and comparison between experimentally observed (blue dots) and computed conditional severing waiting time distribution (red dots) for (B) WT and (C) spr2-2. Image taken from (Nakamura et al., J. Cell Biol., 2018). 
against $11 \%$ of the cases in WT, proving our hypothesis that stability of minus end plays a crucial role in the reorientation mechanism.

\section{Computational approach}

To provide further evidence in support to the hypothesis that the fast minus end depolymerization prevents the cortical microtubule array from reorienting by removing the possible severing spots, we use the experimentally measured parameters for WT and spr2-2 mutant to run stochastic simulations of the model discussed in section 2.1. Given the impossibility to directly measure the probability of shrinkage after severing $q^{-}$for the minus end, we have chosen this parameter such that the time that a microtubule minus end spends on average in either of the two possible states is in agreement with the experimentally measured one, see Table 2.2. Our simulations keep track of the time evolution of a microtubule and all of its descendants created by severing, up to 500 simulated seconds. As well as in the case of the plus end analysis of previous section, this choice is motivated by the fact that we are only interested in the initial stage of the reorientation process, and for more than 500 seconds we can no longer consider the grid of transverse microtubule as a constant background. Results, averaged over $N=5 \times 10^{4}$ different trials, show that even if the extinction probability is smaller in spr2-2 case than in the WT, the amplification of the number of longitudinal microtubules is faster in the WT rather than in the mutant, in agreement with experimental observations, see Figure 2.17. The surprising result that extinction is less likely for spr2-2, depends on the fact that spr2-2 microtubules are in general longer than WT microtubules. Indeed, not only their plus end is more deeply in the unbounded-growth regime, but they also show a decreased shortening effect due to the severing, since the severing probability per crossover is lower than in WT case, see Table 2.3.

Figure 2.17 shows that the amplification process is more efficient for WT. However, the dynamics at the minus end is not the only difference between the two genotypes, since also the dynamics of the plus end is influenced by the presence of SPR2 protein. To isolate the role of the two changes in the dynamics, we build two synthetic in silico mutants: microtubules with WT as a background but with minus end parameters of spr2-2 ( $\mathrm{WT}^{+}$spr2-2 $\left.{ }^{-}\right)$, and microtubules with spr2-2 as a background but with WT parameters for the minus end $\left(\right.$ spr2-2 $\left.2^{+} \mathrm{WT}^{-}\right)$. The most interesting result comes from the observation of the simulations outcome for $\mathrm{WT}^{+}$spr2-2 $^{-}$mutant: indeed, Figure 2.17 shows that amplification is almost nonexistent for such a mutant, with a very high extinction probability as well, confirming the importance of a stable minus end for the efficiency of the amplification process. In contrast, the very fast dynamics of the plus end coupled with the stable minus end of spr2-2 $2^{+} \mathrm{WT}^{-}$, enables a very strong amplification of the number of longitudinal 
microtubules, more than one order of magnitude greater than in the full spr2-2 case.

A

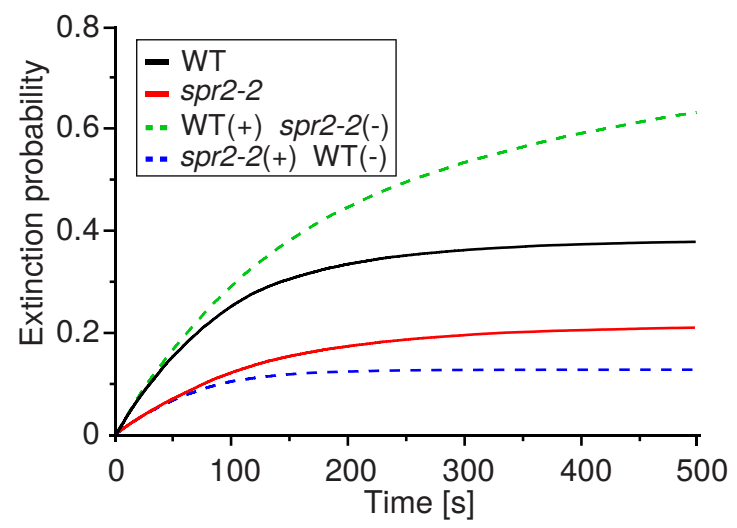

B

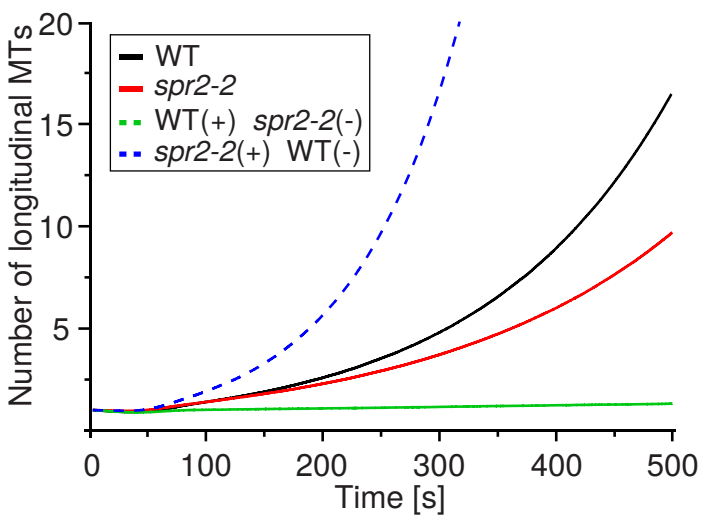

Figure 2.17: Time evolution of (A) the extinction probability, and (B) the number of longitudinal microtubules created by severing for WT, clasp, and for the synthetic mutants $\mathrm{WT}^{+}$spr2-2- ${ }^{-}$, and spr2-2 ${ }^{+} \mathrm{WT}^{-}$. Results are averaged over $N=5 \times 10^{4}$ trials of the same simulation. Image taken from (Nakamura et al., J. Cell Biol., 2018).

To further confirm our results, we perform a statistical analysis of the crossovers resolution (Table 2.3). We observe that spr2-2 and $\mathrm{WT}^{+}$spr2-2 $^{-}$have the same depolymerization probability at a crossover, whilst in WT and spr2-2 ${ }^{+} \mathrm{WT}^{-}$the depolymerization probability is comparable, even if slightly different, showing how the probability of crossover removal due to the shrinkage of microtubules depends more on the minus end than on the plus end dynamics. The same good agreement is exhibited by the probability that the crossover removal occurs because of the shrinkage of either of the two ends, conditional to the fact that the crossover is removed by depolymerization. Intriguingly, experiments and simulations for WT and spr2-2 show a very good agreement for the probability of crossover removal due to the shrinkage of either of the two ends, supporting even more our hypothesis.

However, we notice from Table 2.3, that our model overestimates the probability of severing at a crossover, with respect to the experimental measurements. At the moment, we do not have a full explanation for this overestimate, but we can speculate that it is due to our choice of limiting the dynamics and the severing to only longitudinal microtubules. Indeed, in the experiments we observe crossover removals due to depolymerization of transverse microtubules, as well as severing of the latter. The crossover statistics collected in the experiments take into account 
CLASP and SPR2 promote microtubule reorientation by acting as tip regulatory factors

\begin{tabular}{|ccccc|}
\hline Parameter & Description & WT & spr2-2 & Units \\
\hline$v^{+}$ & Plus end growth speed & 0.105 & 0.105 & $\mu \mathrm{m} \mathrm{s}^{-1}$ \\
$v^{-}$ & Plus end shrinkage speed & 0.195 & 0.21 & $\mu \mathrm{m} \mathrm{s}^{-1}$ \\
$w$ & Minus end shrinkage speed & 0.023 & 0.063 & $\mu \mathrm{m} \mathrm{s}^{-1}$ \\
$r_{c}$ & Plus end catastrophe rate & 0.0063 & 0.0025 & $\mathrm{~s}^{-1}$ \\
$r_{r}$ & Plus end rescue rate & 0.0268 & 0.033 & $\mathrm{~s}^{-1}$ \\
$r_{p s}$ & Minus end pause to shrinking rate & 0.0058 & 0.0138 & $\mathrm{~s}^{-1}$ \\
$r_{s p}$ & Minus end shrinking to pause rate & 0.014 & 0.0016 & $\mathrm{~s}^{-1}$ \\
$t_{p}$ & Fraction of time in pausing state & 0.65 & 0.09 & - \\
$t_{s}$ & Fraction of time in shrinking state & 0.35 & 0.91 & - \\
$p^{+}$ & Rescue after severing $(\|)$ & 0.11 & 0.19 & - \\
$p_{\perp}^{+}$ & Rescue after severing $(\perp)$ & 0.07 & 0.08 & - \\
$q^{+}$ & Minus end shrink probability after severing & 0.35 & 1 & - \\
$d$ & Spacing scale parameter & 0.89 & 0.78 & $\mu \mathrm{m}$ \\
$d_{\text {avg }}$ & Average bundle spacing & 1.39 & 1.28 & $\mu \mathrm{m}$ \\
$k$ & Gamm shape parameter & 7 & 7 & - \\
$\theta$ & Gamma scale parameter & 8.5 & 8.5 & $\mathrm{~s}$ \\
$t_{\text {sev }}$ & Mean severing waiting time & 46.7 & 43.74 & $\mathrm{~s}$ \\
\hline
\end{tabular}

Table 2.2: Minus end dynamics and crossover parameters

crossover resolutions for the entire time of the experiments, i.e. for 30 minutes, and as we have seen before, after 500 seconds from the beginning of the experiment, we can no longer consider the initial transverse array as stable. Thus, including the dynamics of transverse microtubules together with the dynamics of longitudinal could in principle level the discrepancy between experiments and simulations, as regards the fraction of crossovers removed by depolymerization against severing.

A final proof of our hypothesis that the reorientation of the array mainly depends on the stability of microtubule minus end, comes from the analysis of the severing waiting time distribution. In keeping with our assumption that SPR2 does not influence the efficiency of katanin, in our simulation we impose the same intrinsic severing waiting time distribution at the crossovers for both WT and spr2-2. As expected, using the right choice of parameters (Table 2.2) for the Gamma distribution of Eqs. (2.2), our two resultant computed conditional waiting time distributions nicely fit the two experimentally observed waiting time distributions for both WT and spr2-2.

This, together with all other evidence that we provided in this section, confirms that SPR2 protein has no role in promoting or antagonizing the effect of katanin on the severing mechanism. Instead, it promotes the cortical microtubule array reorientation by acting as a protector of the minus end of microtubules, and in this way increasing the lifetime of crossovers as potential microtubule severing sites. 


\begin{tabular}{|c|cc|cccc|}
\hline & Experiments & & Simulations & & & \\
\hline (A) & WT & spr2-2 & WT & spr2-2 & WT $^{+}$spr2-2- & spr2-2 spT- $^{-}$ \\
(B) & 1266 & 1056 & $3.4 \times 10^{6}$ & $1.8 \times 10^{6}$ & $0.5 \times 10^{6}$ & $19.2 \times 10^{6}$ \\
(C) & 0.39 & 0.24 & 0.61 & 0.31 & 0.31 & 0.68 \\
(D) & 0.61 & 0.76 & 0.39 & 0.69 & 0.69 & 0.32 \\
(E) & 0.89 & 0.16 & 0.92 & 0.19 & 0.3 & 0.87 \\
(F) & 0.11 & 0.84 & 0.08 & 0.81 & 0.7 & 0.13 \\
\hline
\end{tabular}

Table 2.3: Crossover resolution in WT, spr2-2, $\mathrm{WT}^{+}$sprD-2 $^{-}$, and spr2-2 ${ }^{+} \mathrm{WT}^{-}$. (A) Genotype, (B) number of analyzed crossovers, (C) severing fraction, (D) depolymerization fraction, (E) fraction of depolymerization from the plus end, (F) fraction of depolymerization from the minus end.

\subsection{Minus end dynamic instability changes steady-state properties of microtubules}

The effect of the dynamic instability at the plus end on the length of microtubules has already been analytically well-studied (Dogterom \& Leibler, Phys. Rev. Lett., 1993). However, a theoretical model that takes into account the dynamic instability of microtubules at both ends has not yet been developed. Therefore, taking our cue from the experimentally observed dynamics of the microtubule minus end, and to better elucidate the effect of this dynamic instability on the length of microtubules before the exposure to blue light - i.e. with no severing events, we set up a stochastic model based on Dogterom-Leibler model in which both ends undergo dynamic instability. In agreement with the experimental observations, the minus end can be either in the shrinking or in the paused state, and it can switch from one state to the other with constant rate. In other words, we use the same model we developed in section 2.1 without severing events, and with a certain nucleation rate $r_{n}$ for new microtubules.

Our goal here is to check whether the dynamic instability at the minus end plays a role in the shape of the steady-state length distribution, or it just changes the length scale of the already known distribution.

\section{Dynamic equations}

Let $m^{\sigma \tau}(t, l)$ be the length distribution of microtubules at time $t$, with length $l$, with plus end in the state $\sigma$, and minus end in the state $\tau$. Plus end state can be either growing $(\sigma=+)$ or shrinking $(\sigma=-)$, and minus end state can be either paused $(\tau=0)$ or shrinking $(\tau=-)$. As experiments have shown, we impose 
$w<v^{+}$(Nakamura et al., J. Cell Biol., 2018). Therefore, the dynamic equations can be written as

$$
\begin{aligned}
\frac{\partial m^{+-}(t, l)}{\partial t}= & -\left(v^{+}-w\right) \frac{\partial m^{+-}(t, l)}{\partial l} \\
& -\left(r_{c}+r_{s p}\right) m^{+-}(t, l)+r_{r} m^{--}(t, l)+r_{p s} m^{+0}(t, l), \\
\frac{\partial m^{+0}(t, l)}{\partial t}= & -v^{+} \frac{\partial m^{+0}(t, l)}{\partial l} \\
& -\left(r_{c}+r_{p s}\right) m^{+0}(t, l)+r_{r} m^{-0}(t, l)+r_{s p} m^{+-}(t, l), \\
\frac{\partial m^{--}(t, l)}{\partial t}= & \left(v^{-}+w\right) \frac{\partial m^{--}(t, l)}{\partial l} \\
& -\left(r_{r}+r_{s p}\right) m^{--}(t, l)+r_{c} m^{+-}(t, l)+r_{p s} m^{-0}(t, l), \\
\frac{\partial m^{-0}(t, l)}{\partial t}= & v^{-} \frac{\partial m^{-0}(t, l)}{\partial l} \\
& -\left(r_{r}+r_{p s}\right) m^{-0}(t, l)+r_{c} m^{+0}(t, l)+r_{s p} m^{--}(t, l),
\end{aligned}
$$

supplemented by the boundary conditions

$$
\begin{gathered}
m^{+-}(t, 0)=0, \\
m^{+0}(t, 0)=\frac{r_{n}}{v^{+}}, \\
\lim _{l \rightarrow \infty} m^{--}(t, l)=0, \\
\lim _{l \rightarrow \infty} m^{-0}(t, l)=0 .
\end{gathered}
$$

The first two boundary conditions come from our choice of nucleating microtubules only with the minus end in the paused state. This is motivated by the experimental observation that microtubule minus end remains attached to the nucleation site until enough katanin is recruited there. Then, the minus end is released from the nucleation site, and treadmilling is enabled (Wasteneys, J. Cell Science, 2002). The last two boundary conditions come from the mathematical requirement of probability density functions to be integrable. Physically, they imply that there are no infinitely long microtubules. 


\section{Steady-state solution}

At first sight, the set of equations (2.3-2.6) seems to be pretty hard to solve. Indeed, to reduce its complexity, we try to find the solution of the steady-state version of the system. Therefore, we denote $\mathbf{m}(l)$ as

$$
\mathbf{m}(l)=\left(\begin{array}{c}
m^{+-}(l) \\
m^{+0}(l) \\
m^{--}(l) \\
m^{-0}(l)
\end{array}\right)
$$

and $\mathbf{L}$ as

$$
\mathbf{L}=\left(\begin{array}{cccc}
-\frac{r_{c}+r_{s p}}{v^{+}-w} & \frac{r_{p s}}{v^{+}-w} & \frac{r_{r}}{v^{+}-w} & 0 \\
\frac{r_{s p}}{v^{+}} & -\frac{r_{c}+r_{p s}}{v^{+}} & 0 & \frac{r_{r}}{v^{+}} \\
-\frac{r_{c}}{v^{-}+w} & 0 & \frac{r_{r}+r_{s p}}{v^{-}+w} & -\frac{r_{p s}}{v^{-}+w} \\
0 & -\frac{r_{c}}{v^{-}} & -\frac{r_{s p}}{v^{-}} & \frac{r_{r}+r_{p s}}{v^{-}}
\end{array}\right) .
$$

Steady-state version of Eqs. (2.3-2.6) can now be written as

$$
\frac{d}{d l} \mathbf{m}(l)=\mathbf{L m}(l) .
$$

The solution of Eq. (2.11) can be achieved with numerical methods, and it will be discussed in Chapter 5. Here, however, we try to find an analytical solution, and to do that we make the further simplification of setting $r_{r}=0$, i.e. we assume that microtubules with the plus end in the shrinking state can not undergo rescues. With this approximation, the equations become

$$
\begin{gathered}
0=-\left(v^{+}-w\right) \frac{d m^{+-}(l)}{d l}-\left(r_{c}+r_{s p}\right) m^{+-}(l)+r_{p s} m^{+0}(l), \\
0=-v^{+} \frac{d m^{+0}(l)}{d l}-\left(r_{c}+r_{p s}\right) m^{+0}(l)+r_{s p} m^{+-}(l), \\
0=\left(v^{-}+w\right) \frac{d m^{--}(l)}{d l}-r_{s p} m^{--}(l)+r_{c} m^{+-}(l)+r_{p s} m^{-0}(l), \\
0=v^{-} \frac{d m^{-0}(l)}{d l}-r_{p s} m^{-0}(l)+r_{c} m^{+0}(l)+r_{s p} m^{--}(l) .
\end{gathered}
$$


By combining Eqs. (2.12) and (2.13), we can show that dynamic instability for microtubules growing at the plus end can be rewritten as uncoupled second order differential equations as

$$
\frac{d^{2} m^{+\tau}(l)}{d l^{2}}+\left(\frac{r_{c}+r_{s p}}{v^{+}-w}+\frac{r_{c}+r_{p s}}{v^{+}}\right) \frac{d m^{+\tau}(l)}{d l}+\frac{r_{c}\left(r_{c}+r_{s p}+r_{p s}\right)}{\left(v^{+}-w\right) v^{+}} m^{+\tau}(l)=0
$$

with $\tau=-, 0$, and with boundary conditions

$$
\begin{gathered}
m^{+-}(0)=0, \\
m^{+0}(0)=\frac{r_{n}}{v^{+}} .
\end{gathered}
$$

Since Eq. (2.16) is a second order differential equation, we need a second boundary condition. Thus, by setting $l=0$ in Eqs. (2.12) and (2.13), we obtain

$$
\begin{gathered}
\left.\frac{d}{d l} m^{+-}(l)\right|_{l=0}=\frac{r_{n}}{v^{+}} \frac{r_{p s}}{v^{+}-w}, \\
\left.\frac{d}{d l} m^{+0}(l)\right|_{l=0}=-\frac{r_{n}\left(r_{c}+r_{p s}\right)}{\left(v^{+}\right)^{2}} .
\end{gathered}
$$

Equation (2.16) is a second order linear differential equation (Polyanin and Zaitsev, 2003), with positive discriminant

$$
\begin{aligned}
\Delta^{2} & \equiv\left(\frac{r_{c}+r_{s p}}{v^{+}-w}+\frac{r_{c}+r_{p s}}{v^{+}}\right)^{2}-4 \frac{r_{c}\left(r_{c}+r_{s p}+r_{p s}\right)}{\left(v^{+}-w\right) v^{+}} \\
& =\left(\frac{r_{c}+r_{s p}}{v^{+}-w}-\frac{r_{c}+r_{p s}}{v^{+}}\right)^{2}+4 \frac{r_{s p} r_{p s}}{\left(v^{+}-w\right) v^{+}}>0 .
\end{aligned}
$$

Therefore, its general solution is

$$
m^{+\tau}(l)=e^{-\frac{1}{2}\left(\frac{r_{c}+r_{s p}}{v^{+}-w}+\frac{r_{c}+r_{p s}}{v^{+}}\right) l}\left[C_{1}^{+\tau} e^{\frac{1}{2} \Delta l}+C_{2}^{+\tau} e^{-\frac{1}{2} \Delta l}\right]
$$

with

$$
\begin{gathered}
C_{1}^{+-}=\frac{r_{n}}{v^{+}} \frac{r_{p s}}{\left(v^{+}-w\right) \Delta}, \\
C_{2}^{+-}=-\frac{r_{n}}{v^{+}} \frac{r_{p s}}{\left(v^{+}-w\right) \Delta}, \\
C_{1}^{+0}=\frac{r_{n}}{v^{+}} \frac{1}{2 \Delta}\left(\Delta+\frac{r_{c}+r_{s p}}{v^{+}-w}-\frac{r_{c}+r_{p s}}{v^{+}}\right),
\end{gathered}
$$




$$
C_{2}^{+0}=\frac{r_{n}}{v^{+}} \frac{1}{2 \Delta}\left(\Delta-\frac{r_{c}+r_{s p}}{v^{+}-w}+\frac{r_{c}+r_{p s}}{v^{+}}\right),
$$

see Figure 2.18. Expressions for $m^{--}(l)$ and $m^{-0}(l)$ can be found by solving a two differential equations system with Eq. (2.21) plugged in Eqs. (2.14) and (2.15).
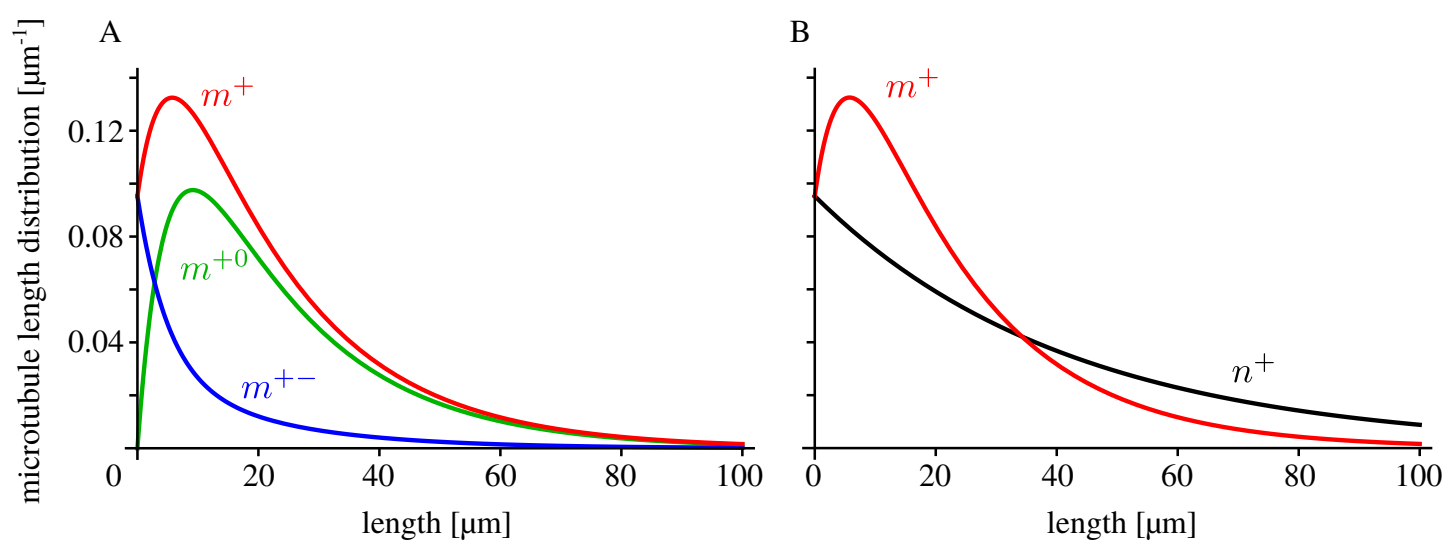

Figure 2.18: Length distribution for microtubules with (A) plus end in growing state and minus end in shrinking (blue), paused (green), and the cumulative distribution for all growing microtubules (red). (B) Comparison between the cumulative distribution for growing microtubules (red), and the exponential distribution $n^{+}(l)=\frac{r_{n}}{v^{+}-\bar{w}} e^{-r_{c} l /\left(v^{+}-\bar{w}\right)}$ for growing microtubule in the Dogterom-Leibler model (black). Parameters used for these distributions are $v^{+}=0.105 \mu \mathrm{ms}^{-1}, w=$ $0.063 \mu \mathrm{m} \mathrm{s}^{-1}, r_{c}=0.0025 \mathrm{~s}^{-1}, r_{p s}=0.0138 \mathrm{~s}^{-1}, r_{s p}=0.0016 \mathrm{~s}^{-1}$, and $r_{n}=$ $0.01 \mathrm{~s}^{-1}$.

\section{Changing the boundary conditions}

Figure 2.18 shows that when we add dynamic instability at the minus end, the steady-state distribution for growing microtubules defined by

$$
m^{+}(l)=m^{+-}(l)+m^{+0}(l),
$$

is no longer the negative exponential distribution of Dogterom-Leibler model of Eq. (1.3). The causes and implications of this observation will be discussed in details in chapter 5 . Here, instead, we want to find a way to recover the general solution of Dogterom and Leibler without changing the main feature of the model, i.e. the presence of dynamic instability at both ends of microtubules, but by acting 
on the boundary conditions at $l=0$. Indeed, since the general solution for the two distributions is the same, we notice that the difference between $m^{+-}(l)$ and $m^{+0}(l)$ arises solely and exclusively from the different boundary conditions of the two functions. Therefore, we ask whether we can find different boundary conditions such that $m^{+-}(l)=\alpha m^{+0}(l)$, with $\alpha \in(0, \infty)$, and, if so, whether we can find an overall shrinking speed for the minus end $\bar{w}$ such that the length distribution of our new system coincides with the length distribution of a Dogterom-Leibler model with growing speed $v^{+}-\bar{w}$. In other words, we have to find the boundary conditions and the parameter $\alpha$ such that

$$
\left\{\begin{array}{l}
C_{1}^{+-}=\alpha C_{1}^{+0} \\
C_{2}^{+-}=\alpha C_{2}^{+0}
\end{array}\right.
$$

We suppose that a fraction $u$ of microtubules are nucleated with the minus end initially in the shrinking state, and consequently, the fraction $1-u$ of microtubules are nucleated in the paused state as in the original model. With these new boundary conditions

$$
\begin{gathered}
m^{+-}(0)=\frac{r_{n}}{v^{+}-w} u, \\
m^{+0}(0)=\frac{r_{n}}{v^{+}}(1-u) \\
\left.\frac{d}{d l} m^{+-}(l)\right|_{l=0}=r_{n}\left[\frac{r_{p s}}{\left(v^{+}-w\right) v^{+}}(1-u)-\frac{r_{c}+r_{s p}}{\left(v^{+}-w\right)^{2}} u\right]
\end{gathered}
$$

and

$$
\left.\frac{d}{d l} m^{+0}(l)\right|_{l=0}=r_{n}\left[\frac{r_{s p}}{\left(v^{+}-w\right) v^{+}} u-\frac{r_{c}+r_{p s}}{\left(v^{+}\right)^{2}}(1-u)\right],
$$

the integration coefficients of Eqs. (2.21) become

$$
\begin{gathered}
C_{1}^{+-}=\frac{r_{n}}{v^{+}\left(v^{+}-w\right)} \frac{1}{2 \Delta}\left[\left(\Delta-\frac{r_{c}+r_{s p}}{v^{+}-w}+\frac{r_{c}+r_{p s}}{v^{+}}\right) v^{+} u+2 r_{p s}(1-u)\right], \\
C_{2}^{+-}=\frac{r_{n}}{v^{+}\left(v^{+}-w\right)} \frac{1}{2 \Delta}\left[\left(\Delta+\frac{r_{c}+r_{s p}}{v^{+}-w}-\frac{r_{c}+r_{p s}}{v^{+}}\right) v^{+} u-2 r_{p s}(1-u)\right], \\
C_{1}^{+0}=\frac{r_{n}}{v^{+}\left(v^{+}-w\right)} \frac{1}{2 \Delta}\left[\left(\Delta+\frac{r_{c}+r_{s p}}{v^{+}-w}-\frac{r_{c}+r_{p s}}{v^{+}}\right)\left(v^{+}-w\right)(1-u)+2 r_{s p} u\right],
\end{gathered}
$$




$$
C_{2}^{+0}=\frac{r_{n}}{v^{+}\left(v^{+}-w\right)} \frac{1}{2 \Delta}\left[\left(\Delta-\frac{r_{c}+r_{s p}}{v^{+}-w}+\frac{r_{c}+r_{p s}}{v^{+}}\right)\left(v^{+}-w\right)(1-u)-2 r_{s p} u\right] .
$$

Conditions (2.27) yield

$$
u=\frac{2 r_{p s}}{2 r_{p s}+\left(\Delta+\frac{r_{c}+r_{s p}}{v^{+}-w}-\frac{r_{c}+r_{p s}}{v^{+}}\right) v^{+}},
$$

and

$$
\alpha=\frac{v^{+}}{2 r_{s p}}\left(\Delta-\frac{r_{c}+r_{s p}}{v^{+}-w} \frac{r_{c}+r_{p s}}{v^{+}}\right) .
$$

Intriguingly, we notice that Eq. (2.36) implies that $C_{2}^{+-}=0=C_{2}^{+0}$. This means that the only possible length distribution such that $m^{+-}(l)=\alpha m^{+0}(l)$ given a certain choice of boundary conditions is the exponential distribution.

By making use of some simple algebra we can show that

$$
\alpha=\frac{u}{1-u} \frac{v^{+}}{v^{+}-w},
$$

and we can eventually rewrite

$$
m^{+}(l)=m^{+-}(l)+m^{+0}(l)=(1+\alpha) m^{+0}(l),
$$

with

$$
m^{+0}(l)=\frac{r_{n}}{v^{+}}(1-u) e^{-\frac{1}{2}\left(\frac{r_{c}+r_{s p}}{v^{+}-w}+\frac{r_{c}+r_{p s}}{v^{+}}-\Delta\right) l} .
$$

\section{Average minus end speed}

To check whether or not a constant average speed $\bar{w}$ for the minus end such that our four state model can be approximated with a two state model with dynamic instability only at the plus end exists, we compare all moments of distribution (2.38) with the moments of the exponential distribution for growing microtubules in the Dogterom-Leibler model, with growing speed $v^{+}-\bar{w}$.

We denote with $M_{j}^{+}$the $j^{\text {th }}$ moment of the distribution $m^{+}(l)$, i.e.

$$
M_{j}^{+}=\int_{0}^{\infty} d l l^{j} m^{+}(l)
$$

and similarly with $N_{j}^{+}$the $j^{\text {th }}$ moment of the distribution

$$
n^{+}(l)=\frac{r_{n}}{v^{+}-\bar{w}} e^{-r_{c} l /\left(v^{+}-\bar{w}\right)} .
$$


We look for $\bar{w}$ such that

$$
N_{j}^{+}=\frac{r_{n}}{j !} \frac{\left(v^{+}-\bar{w}\right)^{j}}{r_{c}^{j+1}}=\frac{r_{n}}{j !} \frac{v^{+}-w+\bar{u} w}{v^{+}\left(v^{+}-w\right)}\left(\frac{2}{\frac{r_{c}+r_{s p}}{v^{+}-w}+\frac{r_{c}+r_{p s}}{v^{+}}-\Delta}\right)^{j+1}=M_{j}^{j},
$$

for every $j=0,1,2, \ldots$ Using some elementary algebra, we can show that

$$
\frac{v^{+}-w+\bar{u} w}{v^{+}\left(v^{+}-w\right)}=\frac{\frac{r_{c}+r_{s p}}{v^{+}-w}+\frac{r_{c}+r_{p s}}{v^{+}}-\Delta}{2 r_{c}}
$$

and if we plug this in Eqs. (2.40), for every $j$ we obtain

$$
\bar{w}=v^{+}-\frac{2 r_{c}}{\frac{r_{c}+r_{s p}}{v^{+}-w}+\frac{r_{c}+r_{p s}}{v^{+}}-\Delta} .
$$

Using this last result we can finally rewrite $m^{+}(l)$ as

$$
\begin{aligned}
m^{+}(l) & =\frac{r_{n}}{2 r_{c}}\left(\frac{r_{c}+r_{s p}}{v^{+}-w}+\frac{r_{c}+r_{p s}}{v^{+}}-\Delta\right) e^{-\frac{1}{2}\left(\frac{r_{c}+r_{s p}}{v^{+}-w}+\frac{r_{c}+r_{p s}}{v^{+}}-\Delta\right) l} \\
& =\frac{r_{n}}{v^{+}-\bar{w}} e^{-\frac{r_{c}}{v^{+}-\bar{w}}} .
\end{aligned}
$$

This result shows that there exists a well defined minus end speed $\bar{w}$ such that we can see the minus end as if it is constantly depolymerizing, even though it undergoes dynamic instability. However Eq. (2.36) shows that this is possible only for a very specific choice of boundary conditions for microtubule nucleation. More specifically, there exists a unique choice of boundary conditions such that there is a minus end speed $\bar{w}$ that makes the steady-state length distribution be a negative exponential, as well as in the Dogterom-Leibler model.

\subsection{Conclusions}

In this chapter, we showed how the control and the regulation of microtubule end stability is crucial for the katanin-based reorientation of the cortical microtubule array of Arabidopsis thaliana cells. With our computational model we have indeed seen how a sufficiently large probability of rescue after severing for the newly-created plus end is a fundamental factor for the amplification of the longitudinal array, as it increases the opportunity for a microtubule to create crossovers with differently oriented microtubules, and therefore the possibility of being severed again. The plus end tracking protein CLASP is responsible for increasing the probability of 
rescue after severing, as it binds to newly-created plus end after severing and it stabilizes them, allowing their polymerization. Moreover, we have shown how a stable minus end is also important for the amplification, as it maintains the severing spots, i.e. microtubule crossovers, for the katanin to act. An unstable minus end, indeed, would remove microtubule crossovers with its depolymerization, decreasing the average lifetime of crossovers, and hence the opportunity to resolve them with a severing event. The minus end tracker SPIRAL2 is important for the stability of microtubule minus ends as it binds there and stabilizes, protecting them against subunit loss. Finally, we introduced a theoretical model for microtubules undergoing dynamic instability at both ends. The model showed that, in general, the steady-state properties of microtubule length change when we add instability at the minus end with respect to the null Dogterom-Leibler model. However, we learned that there exists a unique choice of boundary conditions such that the steady-state length distribution of microtubules with dynamic instability at both ends can be described by a modified Dogterom-Leibler model in which the microtubule minus end always shrinks with a specific shrinking speed that depends on the other dynamic parameters. 


\section{CHAPTER 3}

\section{Probability of rescue after severing defines a critical threshold for microtubule amplification}

In the previous chapter we have shown that the stability of microtubule ends is important for the reorientation of the cortical microtubule array. In particular, we have shown with computer simulations that a high probability of rescue after severing is crucial for enhancing the speed of the amplification of the number of longitudinal microtubules for a different set of mutants. However, the experiments revealed that, at least in the initial phase of reorientation, the microtubules are in the unbounded-growth regime, i.e. the regime when microtubule polymerization prevails over the depolymerization, see Chapter 1 . In this regime, microtubules can in principle live for a very long time, and therefore can be severed a large number of times. Hence, as we observed in Chapter 2, having a non-zero probability of rescue after severing is not strictly necessary for the amplification to occur. Furthermore, the unbounded-growth regime for microtubules amplifying in number cannot be sustained by the cell. Indeed the total amount of tubulin in the cell to drive polymerization is finite, and therefore we can expect that for longer time periods the growing speed of microtubules decreases, making them slow down and enter the bounded-growth regime. It follows that, to completely understand the role of the probability of rescue after severing in the reorientation mechanism, we need to study the system when the reorientation cannot be driven by the fast growth of microtubules. For this reason, in this chapter, we focus on microtubules in the 
bounded-growth regime.

Specifically, we perform an in-depth study of the theoretical model of longitudinal microtubules undergoing dynamic instability in a background of stable transverse microtubules already introduced in Chapter 2. In Section 3.2 we show how, for microtubules in the unbounded-growth regime, the probability of rescue after severing dramatically affects the amplification speed of the cortical array and the success probability of such an amplification. This result can be generalized for the bounded-growth regime as well, provided that the probability of rescue after severing is large enough. Indeed, we show that from the model parameter emerges a critical relationship between probability of succeeding in the amplification mechanism and probability of rescue after severing. In the last section, we calculate the critical threshold that needs to be overcome for the success of the amplification by using a combination of analytical calculations and computer simulations. To achieve this result, we introduce a two-crossovers theory in which the entire background of transverse microtubules is replaced by only two microtubules, and we calculate the contribution of the probability of rescue after severing to the probability that the creation of a crossover leads to a severing event. In order to do that, we develop a new approximated technique to calculate the first-passage time distribution (hereafter FPTD) for microtubules to reach relatively close targets, that can be used for solving first-passage time problems in other systems as well.

\subsection{The model}

\section{Dynamic model}

The model - already introduced in Chapter 2, consists of a single longitudinal microtubule undergoing dynamic instability in the background of transverse microtubules. Every time the longitudinal microtubule crosses a transverse one it creates a crossover where it can be severed. The occurrence of a severing event depends partly on the intrinsic waiting severing time at a crossover of Eq. (2.2) and partly on the dynamic instability of the microtubule. When a severing event occurs, the former long microtubule is now split in two shorter microtubules. Both of them keep undergoing dynamic instability and can create new crossovers. In this way, they have the opportunity to be severed again and to create an offspring of severing-created descendants. After a severing event, the newly-created plus end of the lagging microtubule either is stabilized and enters the growing state with probability $p^{+}$, or the shrinking state with probability $1-p^{+}$. The newly-created minus end of the leading microtubule is now positioned at the severing point in a stable state, whilst no changes are applied to its plus end, see Figure 2.6. 
Probability of rescue after severing defines a critical threshold for microtubule amplification

\begin{tabular}{ccccc}
\hline \hline Parameter & Description & $\begin{array}{c}\text { Numerical value } \\
\text { (bounded) }\end{array}$ & $\begin{array}{c}\text { Numerical value } \\
\text { (unbounded) }\end{array}$ & Units \\
\hline$v^{+}$ & Growth speed & 0.1 & 0.103 & $\mu \mathrm{m} \mathrm{s}^{-1}$ \\
$v^{-}$ & Shrinkage speed & 0.25 & 0.225 & $\mu \mathrm{m} \mathrm{s}^{-1}$ \\
$r_{c}$ & Catastrophe rate & 0.02 & 0.0058 & $\mathrm{~s}^{-1}$ \\
$r_{r}$ & Rescue rate & 0.02 & 0.026 & $\mathrm{~s}^{-1}$ \\
$p^{+}$ & Rescue after severing & Tuned & Tuned & - \\
$d$ & Spacing between neighbors & 1.5 & 1.5 & $\mu \mathrm{m}$ \\
$\theta$ & Gamma scale parameter & 8.5 & 8.5 & $\mathrm{~s}$ \\
$k$ & Gamma shape parameter & 7 & 7 & - \\
\hline \hline
\end{tabular}

Table 3.1: Model parameters.

Contrarily to the previous chapter, here we study the model when the distance between two neighbour transverse microtubules is fixed, in place of being drafted from the probability distribution of Eq. (2.1). In other words, the background of transverse microtubules is replaced by a regular grid with constant spacing $d$ between neighbours.

\section{Microtubule dynamic equations in the interstitial strip}

After the creation of a crossover and before the creation of a second one, the dynamics of the plus end of a microtubule is described by the Dogterom-Leibler model for microtubules undergoing dynamic instability. Notice that the dynamics of the plus end is not influenced by eventual severing events. Therefore, as long as the plus end is at a position $x \in(n d,(n+1) d)$, we can study the property of the correspondent microtubule undergoing dynamic instability in a strip of width $d$ as if its length is $l=x-n d$.

Given our specific interest in studying the properties of the system in both the bounded and the unbounded-growth regime, we have chosen two sets of dynamic parameters: for the bounded-growth case parameters are chosen accordingly to previous observations (Vos et al., Cell Motil. Cytoskel., 2004), while for the unbounded-growth case, both dynamic parameters and grid parameters are those that have been directly measured for the WT case in previous experiments (Lindeboom et al., J. Cell Biol., 2018), see Table 3.1.

\section{Splitting probabilities in the interstitial strip}

If a microtubule plus end impinges on a transverse microtubule, it creates a crossover. After the creation of the crossover the plus end is located at $x \in(n d,(n+1) d)$, and 
as long as this condition is fulfilled the dynamics of microtubules is described by the Dogterom-Leibler model for microtubules with the minus end at $n d$, regardless the occurrence of a severing event. Without any loss of generality, we can therefore set $n=0$ and the length $l=x$.

Due to the dynamic instability of the plus end, the microtubule either reaches length $x=d$ or shrinks back to length $x=0$. The occurrence of either of these events is described by the so-called splitting probabilities $R_{0}^{\sigma}(x)$ and $R_{d}^{\sigma}(x)$. They are the probabilities that a microtubule with initial state $\sigma$ and initial length $x$ arrives first at length 0 or $d$ respectively. Conservation of probability implies that $R_{0}^{\sigma}(x)+R_{d}^{\sigma}(x)=1$.

To formally calculate these probabilities, one can look at the behaviour of a microtubule for an infinitesimal amount of time $\delta t=\delta x / v^{ \pm}$. If the initial length of the microtubule is $x$, then the probability that it reaches the boundary at $d$ is described by

$$
R_{d}^{+}(x)=r_{c} \frac{\delta x}{v^{+}} R_{d}^{-}(x)+\left[1-r_{c} \frac{\delta x}{v^{+}}\right] R_{d}^{+}(x+\delta x),
$$

where, if $\delta t=\delta x / v^{+}$it is small enough, $r_{c} \frac{\delta x}{v^{+}}$is the probability for the microtubule to undergo a catastrophe during $\delta t$. Similarly

$$
R_{d}^{-}(x)=r_{r} \frac{\delta x}{v^{-}} R_{d}^{+}(x)+\left[1-r_{r} \frac{\delta x}{v^{-}}\right] R_{d}^{-}(x-\delta x) .
$$

By combining together Eqs. (3.1) and (3.2) and taking the limit $\delta x \rightarrow 0$, together with the boundary conditions $R_{d}^{+}(d)=1$ and $R_{d}^{-}(0)=0$, we can show (Mulder, Phys. Rev. E, 2012) that

$$
R_{d}^{+}(x)=\frac{e^{x / \bar{l}}-\frac{r_{r} v^{+}}{r_{c} v^{-}}}{e^{d / \bar{l}}-\frac{r_{r} v^{+}}{r_{c} v^{-}}},
$$

and

$$
R_{d}^{-}(x)=\frac{\frac{r_{r} v^{+}}{r_{c} v^{-}}\left(e^{x / \bar{l}}-1\right)}{e^{d / \bar{l}}-\frac{r_{r} v^{+}}{r_{c} v^{-}}}
$$

Similarly, we can obtain

$$
R_{0}^{+}(x)=\frac{e^{d / \bar{l}}-e^{x / \bar{l}}}{e^{d / \bar{l}}-\frac{r_{r} v^{+}}{r_{c} v^{-}}},
$$

and

$$
R_{0}^{-}(x)=\frac{e^{d / \bar{l}}-\frac{r_{r} v^{+}}{r_{c} v^{-}} e^{x / \bar{l}}}{e^{d / \bar{l}}-\frac{r_{r} v^{+}}{r_{c} v^{-}}}
$$


Probability of rescue after severing defines a critical threshold for microtubule amplification

Notice that these expressions hold for both the bounded and the unbounded-growth case. This is a direct consequence of the fact that in a strip both regimes produce a steady-state solution (Govindan \& Spillman, Phys. Rev. E, 2004).

\subsection{Results}

\section{Unbounded-growth regime}

The model introduced in the last chapter has been computationally studied for microtubules in the unbounded-growth regime (Lindeboom et al., J. Cell Biol., 2018) and it shows that the probability of rescue after severing $p^{+}$is more important for the speed of amplification of longitudinal microtubules rather than the intrinsic rescue rate $r_{r}$ of microtubules.

Here, we want to perform an in-depth study of the response to the system to the change of $p^{+}$. We will show that, even though $p^{+}$is crucial for the speed of amplification, it is not required for the occurrence of it.

Our simulations consist of $N=10^{5}$ trials in which a single longitudinal microtubule undergoes dynamic instability in the whole grid of transverse microtubules. For every trial we keep track of the fate of the initial microtubule and its offspring until either no more microtubules are present - i.e. they all have shrunk to length zero, and we call this possible output extinction, or for every trial that did not result in an extinction, the number of microtubules exponentially increases, and we call this second possible output amplification.

Figure 3.1A shows that for our choice of dynamic parameters, the speed of amplification increases monotonically with $p^{+}$. Furthermore, we notice from Figure $3.1 \mathrm{~B}$ that larger values of $p^{+}$correspond to lower extinction probabilities, suggesting that a good stabilization after severing has a double effect: not only it increases the speed of amplification, but also raises the likelihood of the amplification.

It is also interesting to notice that, as $p^{+}$raises, the slope of extinction probability decreases in time in a way that suggests the existence of an asymptotic value for it. In that case, the number of microtubules generated by the first one and its descendants is great enough to prevent the system from extinguishing. We call this asymptotic value ultimate extinction probability. The ultimate extinction probability can be used as an order parameter to estimate how many microtubules are required to make sure that the amplification occurs.

The interesting result that in the unbounded-growth regime even the $p^{+}=0$ case leads to an overall amplification can be explained by an intrinsic property of the regime itself. Indeed, although every severing event shortens the length of the severed microtubule, its plus end is not affected by such an event. Consequently, 

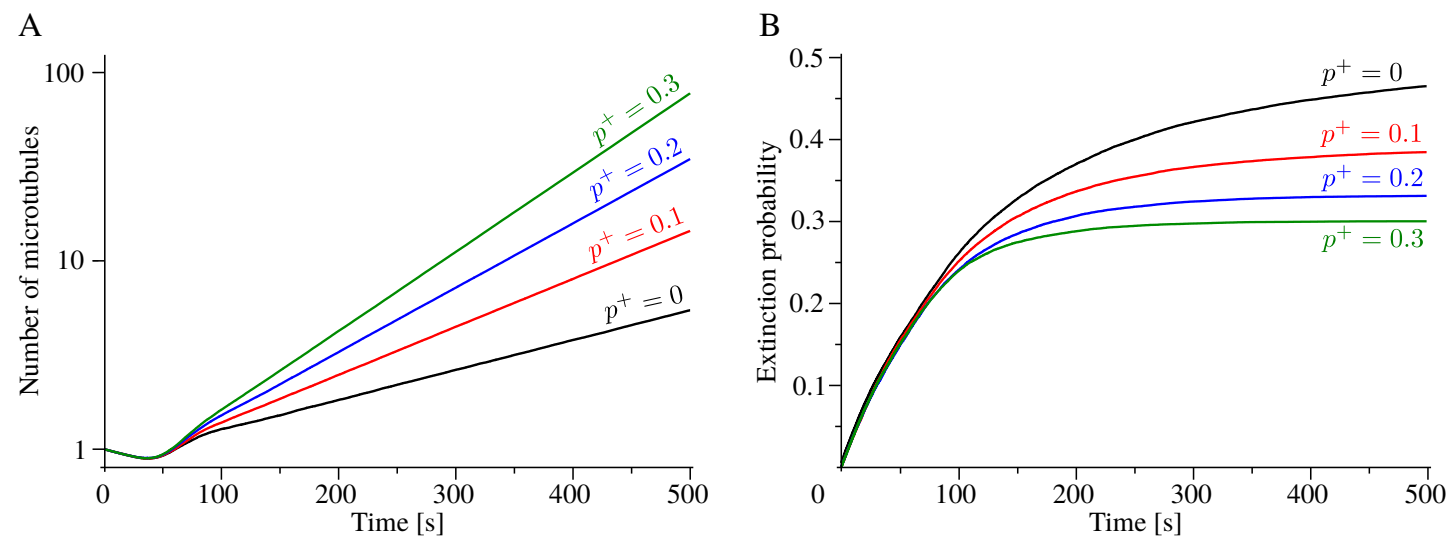

Figure 3.1: (A) Time evolution of the number of longitudinal microtubules for four different values of $p^{+}$. They all show amplification. (B) Extinction probability as a function of time. It represents the fraction of trials in which, after a certain amount of time, all microtubules have completely depolymerized.

the dynamic properties of the leading microtubule are not changed by the severing, and so it applies to the microtubule lifetime as well. Since the average length of microtubules in the unbounded-growth regime grows in time as

$$
V t=\frac{r_{r} v^{+}-r_{c} v^{-}}{r_{r}+r_{c}} t
$$

it follows that the average lifetime of microtubules is infinite (Dogterom \& Leibler, Phys. Rev. Lett., 1993), and hence the probability that at least one microtubule is severed an infinite number of times is 1 .

\section{Bounded-growth regime}

In this section, we address the question whether or not the amplification occurs regardless of $p^{+}$in the bounded-growth regime as well as in the unbounded-growth case. To do so we perform computer simulations for microtubules in the boundedgrowth regime (see Table 3.1 ) to show that $p^{+}$needs to be greater that a certain critical value $p_{c}^{+}$in order have amplification. Moreover, using a combination of computer simulations and analytical calculations we characterize this critical value as a function of the other model parameters. 


\section{Critical point in simulations}

By tuning the probability of rescue after severing $p^{+}$from 0 to 1 , we can observe three different behaviours, see Figure 3.2A: for low values of $p^{+}$the average number of microtubules exponentially decays in time (extinction); for high values of $p^{+}$the number of microtubules exponentially increases (amplification); for a very specific choice of $p^{+}$, i.e. $p^{+} \simeq 0.36$ for our choice of dynamic parameters, the number of microtubules remains constant in time. It follows that this system exhibits criticality in the amplification versus extinction effect as a response to the change of $p^{+}$, and for our choice of model parameters the critical point is $p^{+} \simeq 0.36$. A good choice of order parameter to establish whether the output would be amplification or extinction is the amplification probability, defined as the complementary of the ultimate extinction probability, i.e. the fraction of trials the output of which is amplification. Below the critical point the amplification probability is zero, whilst it is greater than zero otherwise, see Figure 3.2B.
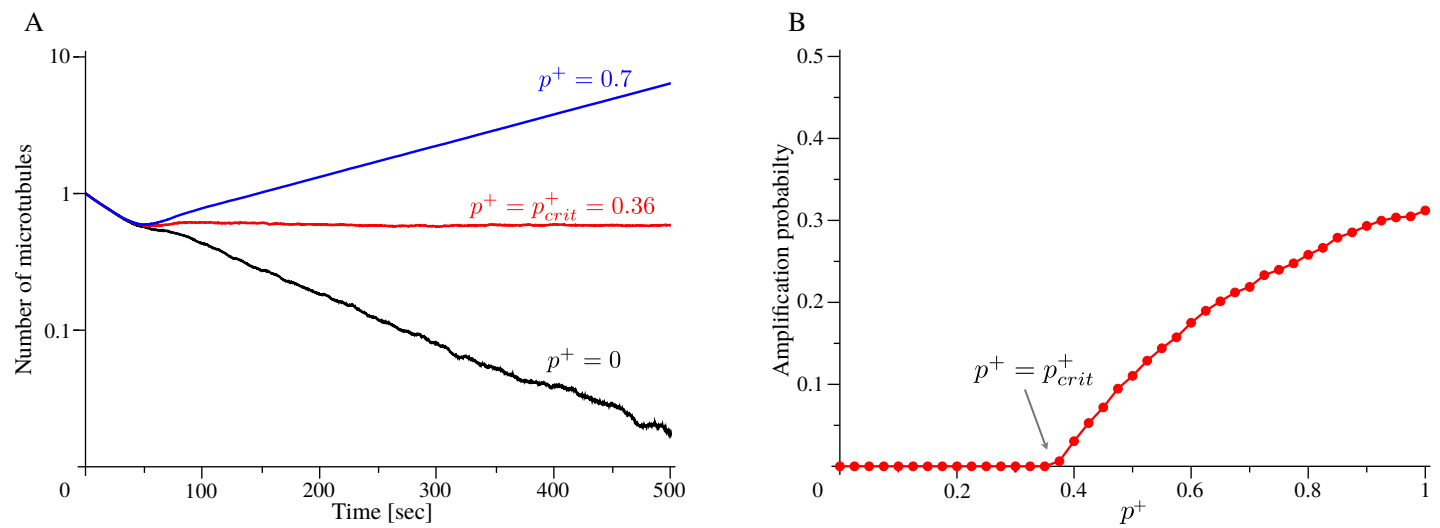

Figure 3.2: (A) Time evolution of the number of longitudinal microtubules for three different values of $p^{+}$. One leads to amplification (blue line), one to extinction (black line), and one corresponds to the critical behaviour (red line). (B) Amplification probability as a function of $p^{+}$. Amplification probability is non-zero for $p^{+}$greater than $p_{\text {crit }}^{+} \simeq 0.36$.

\section{Calculation of the critical point}

The amplification mechanism can be seen as the product of a chain reaction. Indeed, every severing event creates new lagging microtubules that act as intermediates of the reaction during the propagation step (Laidler, Chemical Kinetics, 1987). The newly-created lagging microtubule either shrinks to length zero and die, or it 
generates an offspring of new lagging microtubules. If the size of the offspring is, on average, greater than one, the output is amplification. In other words, suppose that a newly-created microtubule is severed $M$ times (after the first severing event it becomes a leading microtubule). Then amplification occurs if, on average,

$$
M>1 \text {. }
$$

Suppose now that a microtubule (hereafter we refer to it as the leading microtubule) is created by severing with initial length $x=d$. Then, it is initially created in the growing state with probability $p^{+}$, and in the shrinking state with probability $1-p^{+}$. Thus, the size of the offspring of the leading microtubule can be written as $M=p^{+} M^{+}+\left(1-p^{+}\right) M^{-}$, where $M^{\sigma}$ is the size of the offspring of a leading microtubule created in the state $\sigma$. However, it is easy to convince ourselves that $M^{-}=R_{d}^{-}(d) M^{+}$. Indeed, since no severing events can occur if the plus end of the microtubule is at $x \in(0, d)$, the size of the offspring of a microtubule born with length $d$ and in the shrinking state is the same as if it was born in the growing state, provided that the microtubule recovers the initial length $d$, i.e. it creates again the crossover in $d$. Hence, the condition (3.7) can be rewritten as

$$
M=\left[p^{+}+\left(1-p^{+}\right) R_{d}^{-}(d)\right] M^{+}>1 .
$$

By solving the equality related to Eqs. (3.8) we can find the critical value of $p^{+}=p_{\text {crit }}^{+}$above which amplification occurs

$$
p_{\text {crit }}^{+}=\frac{1-R_{d}^{-}(d) M^{+}}{\left(1-R_{d}^{-}(d)\right) M^{+}} .
$$

From this equation, we can identify the two extreme scenarios in which amplification never or always occurs, regardless of $p^{+}$. In the first case, we observe that amplification never occurs if $p_{\text {crit }}^{+}>1$, meaning that the maximum value that $p^{+}$ can reach is not enough to lead to amplification. With simple algebra we can show that condition $p_{\text {crit }}^{+}>1$ is equivalent to the condition

$$
M^{+}<1,
$$

i.e. amplification is impossible if the average size of the offspring of leading microtubules born in the growing state is smaller than 1 . In the second case, we observe that amplification always occurs if $p_{\text {crit }}^{+}<0$, meaning that even without stabilization after severing, dynamic parameters of the model are such that amplification is still possible. This condition is equivalent to

$$
M^{+} R_{d}^{-}(d)>1
$$


or $M^{-}>1$, i.e. amplification occurs every time the average size of the offspring of leading microtubules born in shrinking state is greater than 1 .

Unfortunately we are not able to analytically calculate $M^{+}$, but we can computationally measure it. It is important to notice that, in our discussion, we implicitly assumed that all microtubules are born with initial length $d$, which implies that all severing events happens at the first crossover. We refer to this approximation as first-crossover approximation.

However, given the stochastic nature of the system and of the severing waiting time probability of Eq. (2.2), it is possible that a severing event occurs further in the grid than at the first crossover of a leading microtubule, i.e. the initial length of a newly created microtubule is $x=n d$, with $n>1$. In this case, we have to add to the size of the offspring of the leading microtubule the number of severing events that occur at previous crossovers at its lagging part after the event at $n d$. In other words, if we define $m_{i}$ via $M^{+}=\frac{1}{N} \sum_{i=1}^{N} m_{i}$, as the number of microtubules generated by a single leading microtubule, we denote $s_{i}$ as the number of times its leading part is severed, and $c_{j_{i}}$ as the position of the crossover at which the first severing takes place, with the rule: $c_{j_{i}}=n-1$ if the severing happened at $n d$, then

$$
m_{i}=s_{i}+\sum_{j_{i}=1}^{s_{i}}\left[c_{j_{i}}-b_{c_{j_{i}}}\right],
$$

where $b_{c_{j_{i}}}$ is the number of crossovers resolved by shrinkage of the lagging part of the leading microtubule after the severing at $c_{j_{i}}$. Notice that, since the behaviour of the newly-created plus end of the lagging microtubule depends on $p^{+}$, then $b_{c_{j_{i}}}$ depends on $p^{+}$as well, and so does $m_{i}$. Hence, Eq. (3.9) does not hold anymore. To avoid this complication, in first approximation we replace $m_{i}$ with

$$
m_{i}^{(0)}=s_{i}+\sum_{j_{i}=1}^{s_{i}} c_{j_{i}},
$$

and we directly count this number, see Figure 3.3. If we average $m_{i}^{(0)}$ over $N=10^{5}$ simulations, we eventually find the first approximation for $M^{+}$, i.e. $M_{0}^{+}=$ $2.61 \pm 3.91$. If we plug this number, as well as the parameters of Table 3.1, in Eq. (3.9) we obtain the first estimate of the critical probability of rescue after severing, i.e. $p_{\text {crit },(1)}^{+}=0.316$, against the computationally measured one $p_{\text {crit }}^{+}=0.360$. Table 3.2 shows a comparison between $p_{c r i t,(1)}^{+}$and $p_{c r i t}^{+}$for different sets of dynamic parameters. Notice that, even though our first-crossover approximation provides a reasonable estimate of the critical probability, we systematically underestimate it. 


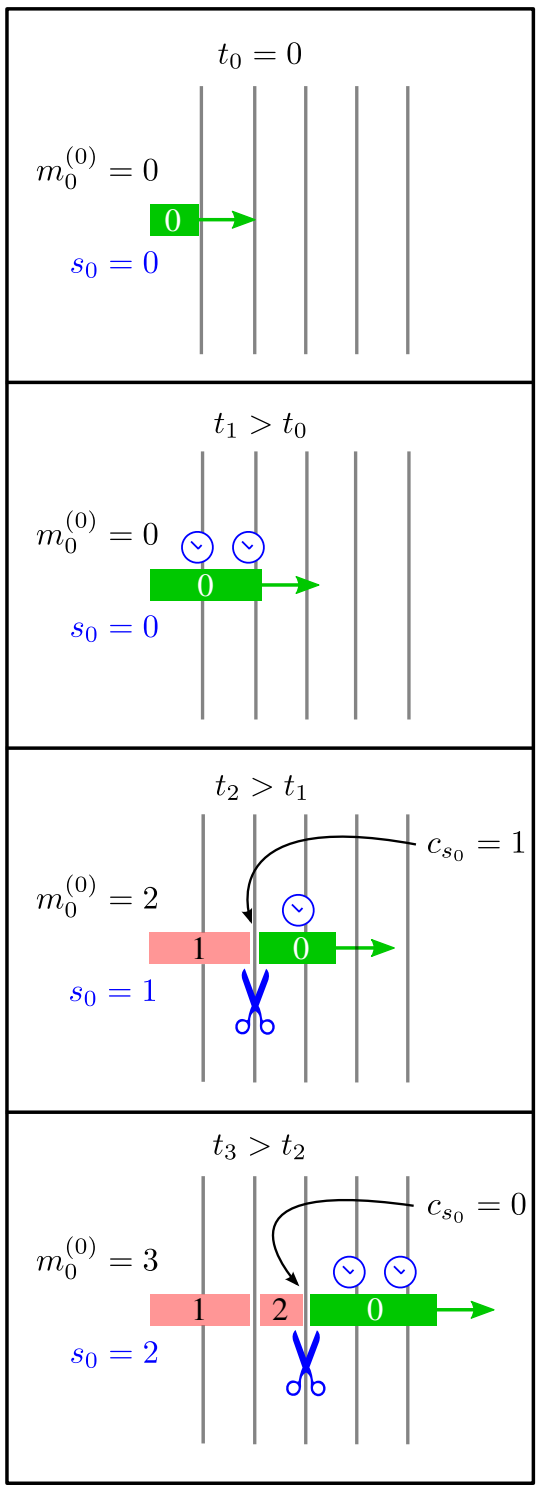

Figure 3.3: Schematic of the count of the size of the offspring $m_{0}^{(0)}$ of a microtubule labelled by 0 created by severing in the growing state. When a crossover is created, the competition severing-shrinking takes place, and if the severing occurs, the counter of the number of severing events $s_{0}$ gains one unit, whilst the size of the offspring gains $1+c_{s_{0}}$. We keep track of the leading microtubule as it can generate other descendants, further increasing $m_{0}^{(0)}$. We do not keep track of the lagging microtubules created by severing. 
In order to have a better estimate of $p_{c r i t}^{+}$, we now try to make the assumption that

$$
b_{c_{j_{i}}}=\left(1-\delta_{c_{j_{i}}, 0}\right) p_{c r}\left(p^{+}\right) \text {, }
$$

where $p_{c r}\left(p^{+}\right)$is the probability that a crossover at $(n-1) d$ is resolved by shrinkage after a severing event occurred at $n d$, conditional to the fact that such a severing event at $n d$ had occurred. The Kronecker function $\delta_{c_{j_{i}}, 0}$ of Eq. (3.14) accounts the fact that, if the severing happens at the first crossover, i.e. $c_{j_{i}}=0$, there is no need of removing one unit from the count of the crossovers that would lead to a severing event.

Notice that with the definition of Eq. (3.14), we are assuming that in case of a severing event at $n d$, only the crossover at $(n-1) d$ can be removed by shrinkage of the newly-created plus end, whilst crossovers at $d, 2 d, \ldots,(n-2) d$ are all resolved by severing. In this case we approximate $m_{i}$ with

$$
m_{i}^{(1)}=s_{i}+\sum_{j_{i}=1}^{s_{i}}\left[c_{j_{i}}-\left(1-\delta_{c_{j_{i}}, 0}\right) p_{c r}\left(p^{+}\right)\right] .
$$

From this equation, we can observe that

$$
\begin{aligned}
\sum_{j_{i}=1}^{s_{i}}\left(1-\delta_{c_{j_{i}}, 0}\right) p_{c r}\left(p^{+}\right) & =p_{c r}\left(p^{+}\right)\left[s_{i}-\left(\delta_{c_{1}, 0}+\delta_{c_{2}, 0}+\cdots+\delta_{c_{s_{i}}, 0}\right)\right] \\
& =p_{c r}\left(p^{+}\right)\left[s_{i}-s_{i}\left\langle\delta_{c_{i}, 0}\right\rangle\right] \\
& =p_{c r}\left(p^{+}\right) s_{i}\left\langle 1-\delta_{c_{i}, 0}\right\rangle
\end{aligned}
$$

where the average value $\left\langle\delta_{c_{i}, 0}\right\rangle$ is calculated over all severing events that a leading microtubule undergoes along its lifetime, and consequently $\left\langle 1-\delta_{c_{i}, 0}\right\rangle$ is the fraction of severing events that a leading microtubule undergoes at $n d$ with $n>1$. If we combine Eqs. (3.13), (3.15), and (3.16) together, and we average over $N$, we obtain

$$
\begin{aligned}
M_{1}^{+} & =\frac{1}{N} \sum_{i=1}^{N} m_{i}^{(1)} \\
& =\frac{1}{N} \sum_{i=1}^{N}\left[s_{i}+\sum_{j_{i}=1}^{s_{i}} c_{j_{i}}-p_{c r}\left(p^{+}\right) s_{i}\left\langle 1-\delta_{c_{i}, 0}\right\rangle\right] \\
& =M_{0}^{+}-p_{c r}\left(p^{+}\right) S \frac{1}{N} \sum_{i=1}^{N}\left\langle 1-\delta_{c_{i}, 0}\right\rangle
\end{aligned}
$$


where $S=\frac{1}{N} \sum_{i=1}^{N} s_{i}$, and where we assumed that the correlation between the number of severing events that occur along the lifetime of a microtubule and the fraction of them that occur at $n d$ with $n>1$ is negligible. In this case, if $N \gg 1$ for the law of large numbers $\frac{1}{N} \sum_{i=1}^{N}\left\langle 1-\delta_{c_{i}, 0}\right\rangle$ is the probability that a microtubule is severed at $n d$ with $n>1$, sampled over all cases in which a severing event had occurred.

By replacing $M^{+}$with $M_{0}^{+}$in Eq. (3.8), we obtain the final amplification condition

$$
M=\left[p^{+}+\left(1-p^{+}\right) R_{d}^{-}(d)\right]\left[M_{0}^{+}-p_{c r}\left(p^{+}\right) S \frac{1}{N} \sum_{i=1}^{N}\left\langle 1-\delta_{c_{i}, 0}\right\rangle\right]>1
$$

Eq. (3.18) contains two quantities that we cannot determine analytically, namely $M_{0}^{+}$and $S$. However, they can be quite easily measured with computer simulations. On the other hand, the probabilities $p_{c r}\left(p^{+}\right)$and $\frac{1}{N} \sum_{i=1}^{N}\left\langle 1-\delta_{c_{i}, 0}\right\rangle$ can be analytically calculated by making use of a two-crossovers theory that we will develop later in this section.

\section{Analytical approach}

In this section we are going to calculate the critical probability of rescue after severing. Our starting point is the inequality (3.18). From the equation associated to that condition, it follows that we need to analytically calculate the probabilities $p_{c r}\left(p^{+}\right)$and $\frac{1}{N} \sum_{i=1}^{N}\left\langle 1-\delta_{c_{i}, 0}\right\rangle$, i.e. the probability that a crossover is removed by the shrinkage of a microtubule induced by a severing event at a following crossover, and the probability that a microtubule is severed at a crossover different than the first one, respectively. In order to do so, and because of the complexity of the model, we make the approximation that the entire grid of transverse microtubules is replaced by just two transverse microtubules. Therefore, we first calculate the FPTD for a longitudinal microtubule to create a crossover with a transverse as we will need it for the formulation of our two-crossovers approximation. Then, we give some analytical results of the one-crossover approximation already introduced in the previous section. Finally, we present the two-crossovers approximation and we show that, with it, we can calculate the critical probability of rescue after severing with a good degree of accuracy. 
Probability of rescue after severing defines a critical threshold for microtubule amplification

\section{The first passage time distribution}

The creation of new crossovers for a microtubule undergoing dynamic instability is intimately linked to a FPTD problem for the same microtubule to reach a target. Here, we face this problem by making use of an approach where we consider all possible legal paths to reach the target, given the knowledge of the time needed to reach it.

The first passage time problem for a microtubule to reach length $x_{1}$ starting from $x_{0}$ in the absence of severing can be seen as a reverse lifetime problem, in the sense that in place of reaching the target at $x_{1}$ the microtubules survives until it arrives at $x_{1}$, as if it was shrinking from $x_{0}$ to $x_{1}$. In this way, the growing speed of the microtubule acts as its shrinking speed, its catastrophe rate as the rescue rate, and viceversa.

Hence, if $L_{\sigma}\left(t \mid x_{1}-x_{0}\right)$ is the lifetime distribution for microtubules with initial length $x_{1}-x_{0}$ and initial state $\sigma$ (see Chapter 1 ), the function that expresses the target reaching at distance $x_{1}-x_{0}$ from the starting point is

$$
L_{\sigma}^{\text {target }}\left(t, x_{1}-x_{0}\right)=\left.L_{\sigma}\left(t \mid x_{1}-x_{0}\right)\right|_{\substack{v^{ \pm} \rightarrow v^{\mp} \\ r_{c} \leftrightarrow r_{r}}},
$$

However, function $L_{\sigma}^{\text {target }}\left(t, x_{1}-x_{0}\right)$ is not yet the FPTD. Indeed, from the definition (3.19) it follows that $\Delta x$ can increase indefinitely before shrinking to 0 . This can happen as a consequence of the decrease of $x_{0}$, the value of which can in principle become negative. Therefore, $L_{\sigma}^{\text {target }}\left(t, x_{1}-x_{0}\right)$ must be re-scaled by the number of paths that reach the target $x_{1}$ at time $t$, without ever shrinking back to $x<x_{0}$, calculated over all possible paths that arrive at $x_{1}$ at time $t$, see Figure 3.4AB. We call these paths legal paths.

For our purpose, the target to reach is a transverse microtubule for the creation of a new crossover, the position of which is at distance $d$ from the starting point, i.e. the previous transverse microtubule. Typically, for the range of values of Table 3.1, every plus end that impinges on a transverse microtubule either does it without any catastrophe, or it undergoes one catastrophe and a subsequent rescue. For the dynamic parameters we are considering, the occurrence of multiple catastrophe-rescue events is very unlikely. Therefore we assume that all paths are either direct - no catastrophes, or indirect - one catastrophe and one rescue.

Given the constant growing and shrinking speeds, the amount of time that a microtubule needs to reach the target at $d$ is given by the time needed to reach it in absence of any catastrophe, added to the time spent from a catastrophe to the moment when the original length before the catastrophe is restored. Mathematically,

$$
T_{d}=\frac{d}{v^{+}}+\Delta x\left(T_{d}\right)\left(\frac{1}{v^{+}}+\frac{1}{v^{+}}\right),
$$


where $T_{d}$ is the first-passage time, and $\Delta x\left(T_{d}\right)$ is the distance walked by the plus end from the catastrophe to the subsequent rescue. From Eq. (3.20) we can find the expression for $\Delta x(T)=\frac{v^{+} v^{-}}{v^{+}+v^{-}}\left(T-\frac{d}{v^{+}}\right)$.

Given the Poisson nature of catastrophe events, if a catastrophe occurs, the probability that it occurs does not depend on the distance to the target. Therefore, the fraction of legal paths can be written as $\Gamma_{0 \rightarrow d}(T)=1-\frac{\Delta x(T)}{d}$, and finally the FPTD as

$$
F_{0 d}(t)=L_{\sigma}^{\text {target }}(t, d) \Gamma_{0 \rightarrow d}(t) \Theta\left[d\left(\frac{2}{v^{+}}+\frac{1}{v^{-}}\right)-t\right],
$$

where the Heaviside theta is imposed to allow at most one catastrophe-rescue event. Indeed $t=d\left(\frac{2}{v^{+}}+\frac{1}{v^{-}}\right)$is the amount of time that a microtubule needs to almost reach the target yet without touching it, i.e. $\frac{d}{v^{+}}$, then undergo a catastrophe and almost shrink to length 0 , i.e. $\frac{d}{v^{-}}$, and finally undergo a rescue and grow until the target is reached, $\frac{d}{v^{+}}$.

In order to separate direct paths from indirect paths, it is convenient to split $F_{0 d}(t)$ in two parts, and rewrite it as

$$
F_{0 d}(t)=\delta\left(t-\frac{d}{v^{+}}\right) e^{-r_{c} t}+f_{0 d}(t)
$$

where the term proportional to delta accounts direct paths, while $f_{0 d}(t)$ accounts indirect. From Section 3.1 we know that it is not granted that the microtubule reaches the target but it succeeds with probability $R_{d}^{+}(x)$. Therefore $F_{0 d}(t)$ is normalized to $R_{d}^{+}(x)$ and, as a consequence, the relation

$$
\int_{0}^{\infty} d t f_{0 d}(t)=R_{d}^{+}(0)-e^{-\frac{r_{c} d}{v^{+}}}
$$

holds.

We run $N=10^{6}$ simulations of microtubule plus ends undergoing dynamic instability in a strip of length $d$, and we create the histogram of the arrival times for the $N \times R_{0 d}^{+}$plus ends that reach the target. Figure 3.4CD shows that the approximation of only one catastrophe-rescue event is a good approximation when the target is relatively close compared to the dynamic parameters of the microtubules, while it apparently fails when the target is more distant, see Figure $3.4 \mathrm{EF}$. However, it is convenient to underline that for $d \gg \bar{l}$ we can observe a very few arrivals at the target, since from Eq. (3.3) we notice that the arrival probability $R_{d}^{+}(0)$ decays as $e^{-d / \bar{l}}$. On the other hand, in the unbounded-growth regime, since the fraction $1-\frac{r_{c} v^{-}}{r_{r} v^{+}}$of plus ends always arrives at the target, for distant targets the approximation of one catastrophe-rescue event is no longer good. 

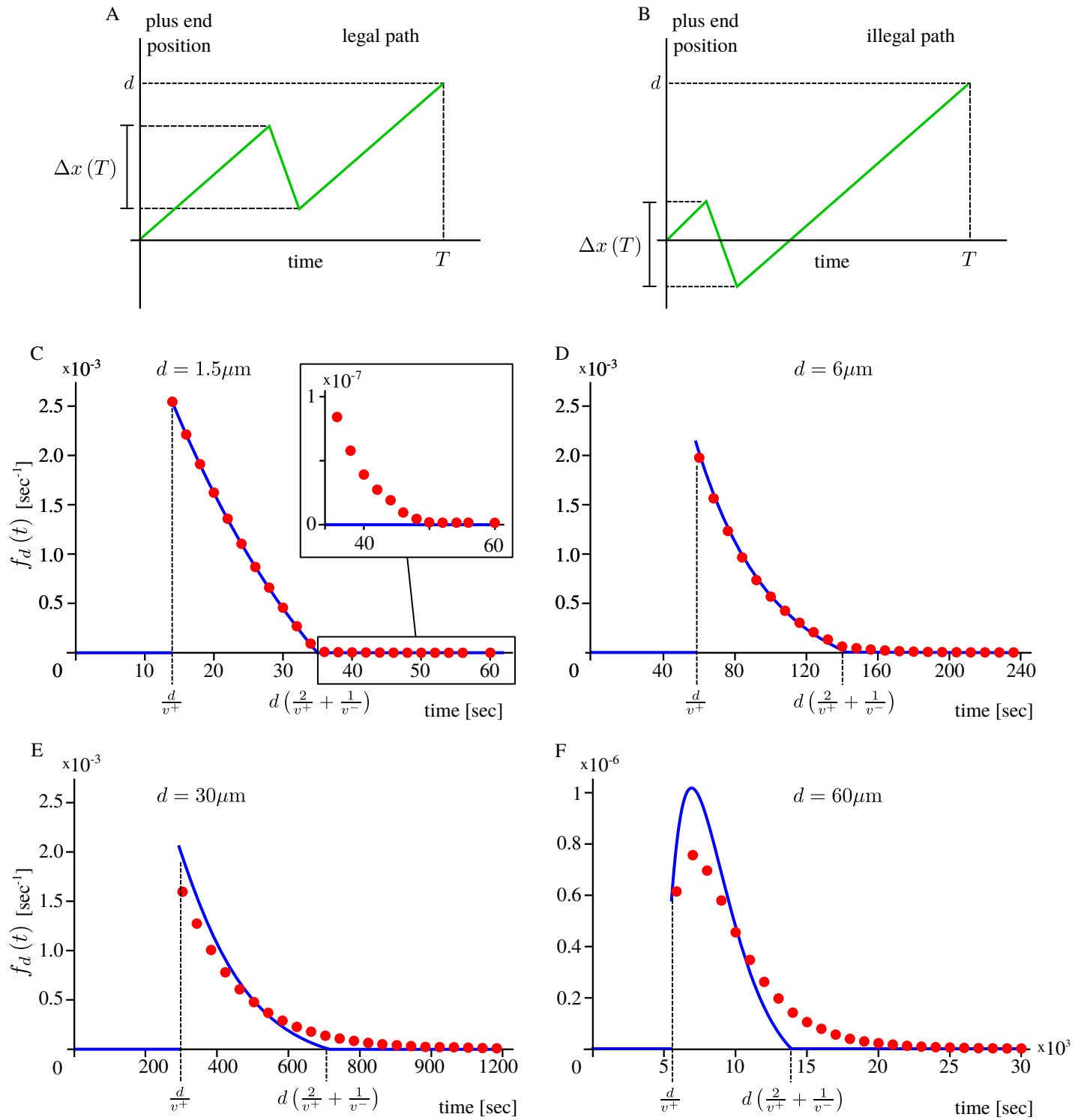

Figure 3.4: (A) Legal and (B) illegal path for a microtubule to reach the target at a distance $d$ in a first passage time $T$. Only one catastrophe and one rescue are allowed. (C-F) Comparison between simulations (red dots) and theory (blue line) for the non-direct part $f_{d}(t)$ of the first passage time distribution. (B, C) Our theory nicely fits simulations for relatively close targets $(d=1.5 \mu \mathrm{m}, d=6 \mu \mathrm{m})$, $(\mathrm{D}, \mathrm{E})$ while it apparently fails for more distant targets $(d=30 \mu \mathrm{m}, d=60 \mu \mathrm{m})$. 


\section{One-crossover theory}

Naïvely, one can think that once a crossover is created the probability $p_{\text {sev }}^{(1)}$ of resolving it with a severing event is given by the competition between two independent events: microtubule lifetime, expressed by the random variable $T_{+}(x)$ with density function given by Eq. (1.7), and severing waiting time at the crossover, with random variable $\tau_{d}$ and density function defined in Eq. (2.2). Then, if we define the random variable $t=\tau_{d}-T_{0}$, we can calculate its probability density function by using the relation

$$
P_{z}(z=x+y)=\int_{-\infty}^{+\infty} d z^{\prime} P_{x}\left(z-z^{\prime}\right) P_{y}\left(z^{\prime}\right)
$$

where $P_{i}$ is the probability density function of the random variable $i=x, y, z$, and $x$ and $y$ are independent random variables (Stuart \& Ord, 1994). In our case, the probability density function is

$$
P_{\tau_{d}-T_{+}(0)}(t)=\int_{-\infty}^{+\infty} d t^{\prime} W_{k, \theta}\left(t+t^{\prime}\right) L_{+}\left(t^{\prime} \mid 0\right)
$$

Hence, the probability that the event "severing" occurs before the event "return" is $\operatorname{Pr}[t<0]=\int_{-\infty}^{0} d t P_{\tau_{d}-T_{+}(0)}(t)$. This probability is not yet the probability of resolving a crossover with a severing event: indeed, microtubules in the unboundedgrowth regime have a finite probability of growing indefinitely. In that case, the microtubule lifetime $T_{+}(0) \rightarrow \infty$, whereas Eq. (3.25) only refers to those of which it is finite. Therefore, the probability of resolving a crossover with a severing event is

$$
p_{\text {sev }}^{(1)}=S_{+}(\infty \mid 0)+\left[1-S_{+}(\infty \mid 0)\right] \int_{-\infty}^{0} d t P_{\tau_{0}-T_{+}(0)}(t)
$$

Consequently the probability of resolving a crossover with a shrinkage

$$
p_{\text {shrink }}^{(1)}=1-p_{\text {sev }}^{(1)} \text {. }
$$

However, with this approach we are not considering the number of crossovers removed by shrinkage after the severing at a second crossover, and as a consequence the dependency on $p^{+}$. In other words, some microtubules that would have been severed at the crossover at $d$, may not be severed there anymore, because a severing at the crossover $n d$, with $n>1$, can in principle shorten their lifetimes, and make them shrink below the crossover at $d$ before the severing occurs, see Figure 3.5. 
Probability of rescue after severing defines a critical threshold for microtubule amplification

\section{Two-crossovers theory}

In order to take into account the influence of a crossover on the resolution of the previous one, we calculate the probability of resolving a crossover with a severing event in a scenario in which we have only two transverse microtubules, at positions $d$ and $2 d$. We make the further approximation that the same microtubule cannot be severed two times at the same crossover. We denote the probability of having a severing event at $d$ as $p_{\text {sev }}^{(2)}$, and consequently we define the probability of resolving a crossover with shrinkage as $p_{\text {shrink }}^{(2)}=1-p_{\text {sev }}^{(2)}$.

Figure 3.5B shows the three distinct ways in which the newly-created crossover at $d$ can be resolved by shrinkage or severing: 1) the plus end shrinks (is severed) before reaching $2 d, 2$ ) the plus end shrinks (is severed) after reaching $2 d$ but before being severed there, 3 ) the plus end shrinks (is severed) after reaching $2 d$ and after being severed there. The third case bears a dependency on $p^{+}$because the dynamic instability of the newly-created plus end of the lagging microtubule is altered by the severing event.

We first notice that $p_{\text {shrink }}^{(2)}$ can be split in two probabilities, i.e. $p_{\text {shrink }}^{(2)}=$ $q_{\text {sev }}+q_{\text {shrink }}$, where $q_{\text {sev }}$ is the probability of shrinkage after severing at $2 d$, while $q_{\text {shrink }}$ is the probability of shrinkage without any severing. Furthermore, since $q_{\text {sev }}$ depends on the dynamic behaviour of the microtubule just after the severing event, it carries a dependency on $p^{+}$and can be split again in $q_{\text {sev }}\left(p^{+}\right)=$ $p^{+} q_{s e v,+}+\left(1-p^{+}\right) q_{s e v,-}$, where $q_{s e v, \sigma}$ is the probability of shrinkage after being severed at $2 d$ with the newly-created plus end in the state $\sigma$.

As regards the probability $q_{\text {shrink }}$ that microtubules shrink below $d$ without being severed there, we observe that such a probability accounts all cases in which crossovers are resolved by shrinkage in absence of the crossover at $2 d$, i.e. $p_{\text {shrink }}^{(1)}$, except for those cases in which microtubules that in principle would have shrunk back, do not have enough time to do so because they are severed at $2 d$. We describe this situation with the probability $q_{n s}$, and hence $q_{\text {shrink }}=p_{\text {shrink }}^{(1)}-q_{n s}$.

Therefore, the final expressions for the probabilities of resolving a crossover with a severing and with a shrinkage are

$$
\begin{gathered}
p_{\text {shrink }}^{(2)}\left(p^{+}\right)=p_{\text {shrink }}^{(1)}-q_{n s}+q_{\text {sev }}\left(p^{+}\right), \\
p_{\text {sev }}^{(2)}\left(p^{+}\right)=p_{\text {sev }}^{(1)}+q_{n s}-q_{\text {sev }}\left(p^{+}\right),
\end{gathered}
$$

see Figure 3.6. Notice that $q_{\text {sev }}\left(p^{+}\right)$can be rewritten as

$$
q_{\text {sev }}\left(p^{+}\right)=q_{\text {sev },-}-\left(q_{\text {sev },-}-q_{\text {sev },+}\right) p^{+},
$$

where the term in the braces is always positive. Indeed, all microtubules with a growing plus end after severing have to first return back to the severing point in the 


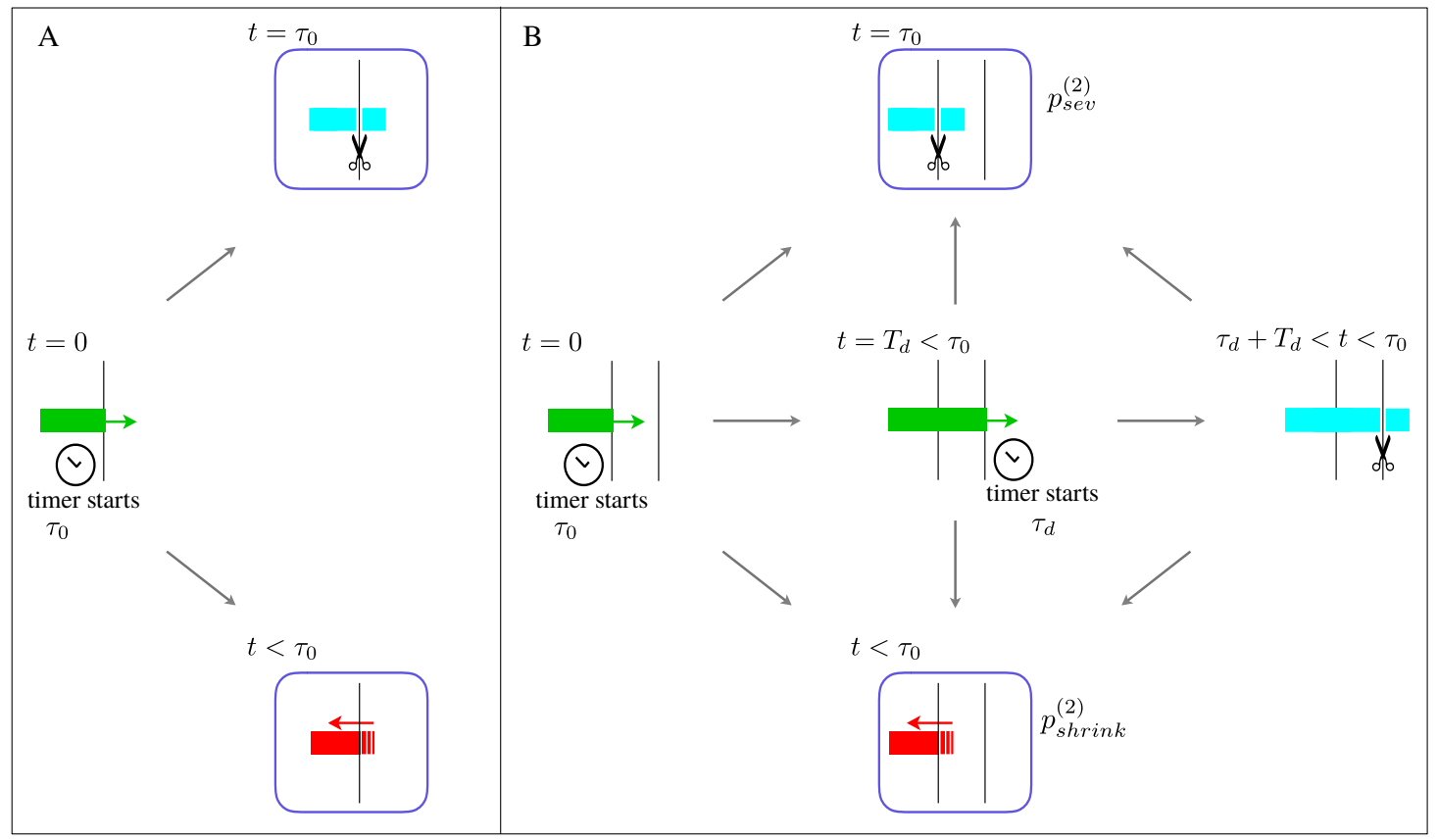

Figure 3.5: Schematic of the one-crossover theory (A). The newly-created crossover can be resolved either by the shrinkage of the plus end below the crossover itself (lower blue square), or by the severing at crossover (upper blue square). Schematic of the two-crossovers theory (B). The first crossover created can be resolved either by the shrinkage of the longitudinal microtubule with probability $p_{\text {shrink }}^{(2)}$ (sum of all paths that bring to lower blue square), or by the severing at crossover with probability $p_{\text {sev }}^{(2)}$ (sum of all paths that bring to upper blue square). Whether the severing at the first crossover occurs or not also depends on what happens at the second crossover: a severing event at the second crossover alters the dynamic instability of the lagging microtubule, and hence its probability of shrinking before being severed at the first crossover. 
shrinking state before completing the shrinkage. In other words, since a microtubule initially in the growing state takes more time to completely depolymerize than a microtubule in the shrinking state, its probability of resolving the crossover at $d$ before being severed there is smaller than in the opposite case. Consequently, the probability $p_{\text {sev }}^{(2)}\left(p^{+}\right)$of resolving a crossover with a severing event grows linearly with $p^{+}$.

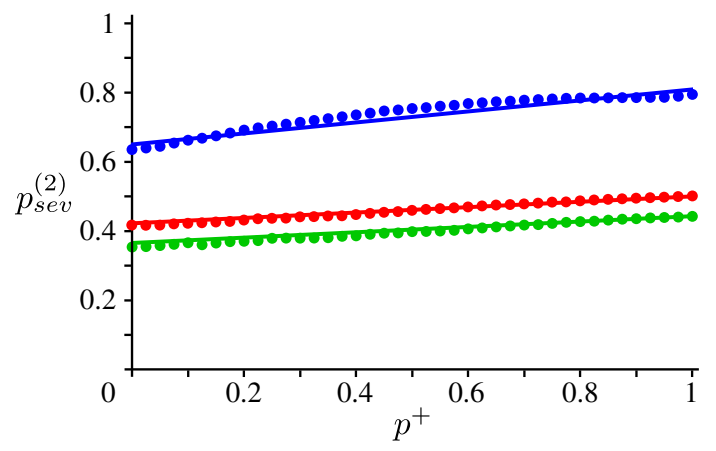

Figure 3.6: Probability of severing at a crossover. Comparison between simulations in the whole grid (dots) and two crossovers severing probability $p_{\text {sev }}^{(2)}$ (straight lines) for three different sets of dynamic parameters reveals that the two-crossovers theory very well approximates the behaviour of the severing probability at a crossover as a response to the change of $p^{+}$. In particular, we use both bounded-growth regime parameters, respectively $v^{+}=0.1 \mu \mathrm{m} \mathrm{s}^{-1}, v^{-}=0.25 \mu \mathrm{m} \mathrm{s}^{-1}, r_{c}=0.02 \mathrm{~s}^{-1}$, $r_{r}=0.02 \mathrm{~s}^{-1}$ (red dots and red line), and $v^{+}=0.11 \mu \mathrm{m} \mathrm{s}^{-1}, v^{-}=0.275 \mu \mathrm{m} \mathrm{s}^{-1}$, $r_{c}=0.022 \mathrm{~s}^{-1}, r_{r}=0.01 \mathrm{~s}^{-1}$ (green dots and green line), and unbounded-growth regime parameters $-v^{+}=0.1 \mu \mathrm{m} \mathrm{s}^{-1}, v^{-}=0.25 \mu \mathrm{m} \mathrm{s}^{-1}, r_{c}=0.006 \mathrm{~s}^{-1}, r_{r}=0.02 \mathrm{~s}^{-1}$ (blue dots and blue line).

With this two-crossovers theory, we can finally give a new estimate of the critical probability of rescue after severing by calculating the probabilities $p_{c r}\left(p^{+}\right)$ and $\frac{1}{N} \sum_{i=1}^{N}\left\langle 1-\delta_{c_{i}, 0}\right\rangle$ of Eq. (3.18). In order to do that, we first define $p_{2 d}$ as the probability to have a severing event at $2 d$ before an eventual severing event at $d$. The proper expression for $p_{2 d}$, as well as those for $q_{s e v, \sigma}$ and $q_{n s}$ can be found in the appendix.

We now define the three events $A, B$, and $C$ as

$A=$ shrinkage of microtubule below $d$ after severing at $2 d$,

$B=$ severing event at $2 d$ before severing event at $d$,

$C=$ severing event at either $d$ or $2 d$. 
The three events are nested as $A \subset B \subset C$, and their probabilities are $\operatorname{Pr}(A)=$ $q_{\text {sev }}\left(p^{+}\right), \operatorname{Pr}(B)=p_{2 d}$, and $\operatorname{Pr}(C)=p_{\text {sev }}^{(2)}\left(p^{+}\right)-q_{\text {sev }}\left(p^{+}\right)+p_{2 d}=p_{\text {sev }}^{(1)}-q_{n s}+p_{2 d}$.

Thus, in this two-crossovers theory, it holds

$$
\begin{aligned}
p_{c r}\left(p^{+}\right) & =\operatorname{Pr}(A \mid B)=\frac{\operatorname{Pr}(A \cap B)}{\operatorname{Pr}(B)} \\
& =\frac{\operatorname{Pr}(A)}{\operatorname{Pr}(B)}=\frac{q_{\text {sev }}\left(p^{+}\right)}{p_{2 d}},
\end{aligned}
$$

and

$$
\begin{aligned}
\frac{1}{N} \sum_{i=1}^{N}\left\langle 1-\delta_{c_{i}, 0}\right\rangle & =\operatorname{Pr}(B \mid C)=\frac{\operatorname{Pr}(B \cap C)}{\operatorname{Pr}(C)} \\
& =\frac{\operatorname{Pr}(B)}{\operatorname{Pr}(C)}=\frac{p_{2 d}}{p_{\text {sev }}^{(1)}-q_{n s}+p_{2 d}} .
\end{aligned}
$$

With these probabilities, the resolution of the equality associated to inequality (3.18) of Section 3.2 leads to $p_{c r i t,(2)}^{+}$, that is

$$
\begin{aligned}
& p_{c r i t,(2)}^{+}=\frac{1}{2 S \Delta q_{s e v} \beta\left(1-R_{d}^{-}(d)\right)} \\
& \times\left\{\left(M_{0}^{+}-S \alpha \beta\right)\left(1-R_{d}^{-}(d)\right)-S \Delta q_{s e v} \beta R_{d}^{-}(d)\right. \\
& \left.\quad+\sqrt{\left[\left(M_{0}^{+}-S \alpha \beta\right)\left(1-R_{d}^{-}(d)\right)+S \Delta q_{s e v} \beta R_{d}^{-}(d)\right]^{2}-4 S \Delta q_{s e v} \beta\left(1-R_{d}^{-}(d)\right)}\right\}
\end{aligned}
$$

where

$$
\begin{gathered}
\alpha=p_{\text {sev }}^{(1)}+q_{n s}-q_{\text {sev },-}, \\
\beta=\frac{1}{p_{\text {sev }}^{(1)}-q_{n s}+p_{2 d}}, \\
\Delta q_{\text {sev }}=q_{\text {sev },-}-q_{\text {sev },+} .
\end{gathered}
$$

Therefore, we can compare $p_{\text {crit, (2) }}^{+}$with $p^{+}$for the reference values of Table 3.1 and for other sets of dynamic parameters, see Table 3.2.

Table 3.2 shows a very good agreement between our predicted critical probability of rescue after severing in the two-crossovers approximation and the critical probability obtained with our simulations in the whole grid of transverse microtubules, confirming our hypothesis that in order to study the critical properties of 
Probability of rescue after severing defines a critical threshold for microtubule amplification

\begin{tabular}{cccc|ccc}
\hline \hline \multicolumn{3}{c}{ Dynamic parameters } & $\begin{array}{c}\text { Critical point } \\
\text { (simulations) }\end{array}$ & $\begin{array}{c}\text { Critical point } \\
\text { (1-cross. theory) }\end{array}$ & $\begin{array}{c}\text { Critical point } \\
(2 \text {-cross. theory) }\end{array}$ \\
\hline$v^{+}$ & $v^{-}$ & $r_{c}$ & $r_{r}$ & $p_{c r i t}^{+}$ & $p_{\text {crit },(1)}^{+}$ & $p_{\text {crit },(2)}^{+}$ \\
$\mu \mathrm{m} \mathrm{s}^{-1}$ & $\mu \mathrm{m} \mathrm{s}^{-1}$ & $\mathrm{~s}^{-1}$ & $\mathrm{~s}^{-1}$ & - & - & - \\
\hline 0.10 & 0.250 & 0.020 & 0.020 & 0.360 & $0.316(0.122)$ & $0.361(0.003)$ \\
0.08 & 0.275 & 0.016 & 0.022 & 0.338 & $0.297(0.121)$ & $0.337(0.003)$ \\
0.15 & 0.225 & 0.020 & 0.020 & 0.142 & $0.108(0.239)$ & $0.144(0.014)$ \\
0.10 & 0.250 & 0.030 & 0.015 & 0.882 & $0.819(0.071)$ & $0.864(0.020)$ \\
0.10 & 0.250 & 0.015 & 0.030 & 0.089 & $0.068(0.236)$ & $0.103(0.157)$ \\
0.10 & 0.250 & 0.030 & 0.015 & 0.800 & $0.733(0.084)$ & $0.780(0.025)$ \\
0.10 & 0.275 & 0.020 & 0.030 & 0.285 & $0.240(0.158)$ & $0.285(0.000)$ \\
0.10 & 0.250 & 0.010 & 0.020 & 0.054 & $0.041(0.241)$ & $0.066(0.222)$ \\
0.08 & 0.225 & 0.015 & 0.025 & 0.208 & $0.179(0.139)$ & $0.213(0.024)$ \\
0.12 & 0.225 & 0.020 & 0.025 & 0.175 & $0.140(0.200)$ & $0.179(0.023)$ \\
0.08 & 0.250 & 0.002 & 0.020 & 0.510 & $0.455(0.108)$ & $0.497(0.025)$ \\
\hline \hline
\end{tabular}

Table 3.2: Comparison $p_{\text {crit,(2) }}^{+}$vs $p_{\text {crit }}^{+}$for different sets of dynamic parameters. All other model parameters are those of Table 3.1. The numbers in the braces represent the relative error between the measured quantity $p_{c r i t}^{+}$and the theoretical predictions $p_{c r i t,(1)}^{+}$and $p_{c r i t,(2)}^{+}$.

the system, we can approximate the entire grid of transverse microtubules with just two of them without any substantial loss of accuracy. Furthermore, this result is in agreement with our choice of considering the severing of microtubules only possible at the first two crossovers that they have along their length.

\subsection{Conclusions}

Our aim was to study the response of the cortical microtubule array to a change in the probability of rescue for the newly-created plus ends after severing. To do that, we first introduced a stochastic model of microtubules undergoing dynamic instability in a grid of stable transverse microtubules, and we observed that the system responds to the change of such a probability in two distinct ways: by increasing the speed of amplification and by reducing the extinction probability. We observed that, although for microtubules in the unbounded-growth regime the amplification is on average always reached even when the probability of rescue after severing is zero, in the bounded-growth regime such a probability is required to be greater than a certain critical threshold in order to have amplification. The difference between the two regimes comes from the fact that in the unboundedgrowth case some microtubules have an infinitely long lifetime, and hence they are 
severed an infinite amount of times, allowing the amplification to occur. Next, we identified the critical relationship between probability of rescue after severing and the rest of the dynamic parameters of the model. In order to do that, we introduced a two-crossovers theory in which the entire grid of transverse microtubules is replaced by only two of them. The introduction of this theory carried the development a novel technique to calculate the FPTD of microtubules to reach a relatively close target based on the sum over all possible paths that reach the target without a complete depolymerization of the microtubule. This technique allowed us to estimate the critical probability of rescue after severing in our two-crossovers approximation with high degree of accuracy, and has the potential to be used to calculate the FPTD for other systems as well.

\subsection{Appendix}

\section{Calculation of the severing probability at a crossover}

In order to calculate $q_{s e v, \sigma}$ we define the following random variables: severing waiting time at $d$ as $\tau_{d}$ and at $2 d$ as $\tau_{2 d}$ with density function of Eq. (2.2), FPT at second crossover $T_{d}$ with density function of Eq. (3.21), lifetime $T_{\sigma}(x)$ of a microtubule with initial state $\sigma$ and initial length $x$, and severing waiting time $\widetilde{\tau}_{2 d}$ at $2 d$ with the further requirement that the severing event occurs before the removal of crossover at $2 d$ due to shrinkage. Given the independence of the two events, it follows that the cumulative function $\Phi_{\widetilde{\tau}_{2 d}}(t)=\operatorname{Pr}\left[\widetilde{\tau}_{2 d}<t\right]$ of $\widetilde{\tau}_{2 d}$ can be rewritten as

$$
\begin{aligned}
\Phi_{\widetilde{\tau}_{2 d}}(t) & =\operatorname{Pr}\left[\left(\tau_{2 d}<t\right) \cap\left(\tau_{2 d}<T_{+}(0)\right)\right] \\
& =\frac{1}{Z_{W}} \int_{0}^{t} d t^{\prime} W_{k, \theta}\left(t^{\prime}\right) \int_{t^{\prime}}^{\infty} d t^{\prime \prime} L_{+}\left(t^{\prime \prime} \mid 0\right),
\end{aligned}
$$

where

$$
Z_{W}=\int_{0}^{\infty} d t W_{k, \theta}(t) \int_{t}^{\infty} d t^{\prime} L_{+}\left(t^{\prime} \mid 0\right)
$$

Therefore, the probability density function $\widetilde{W}_{k, \theta}(t)$ of $\widetilde{\tau}_{2 d}$ is

$$
\widetilde{W}_{k, \theta}(t)=\frac{1}{Z_{W}} W_{k, \theta}(t) \int_{t}^{\infty} d t^{\prime} L_{+}\left(t^{\prime} \mid 0\right),
$$

where we used the definition of probability density function, $\widetilde{W}_{k, \theta}(t)=\frac{d}{d t} \Phi_{\widetilde{\tau}_{2 d}}(t)$.

The probabilities $q_{s e v, \sigma}$ can be seen as the probability that the microtubule reaches $2 d$, it is severed there with newly-created plus end in the state $\sigma$, and 
finally shrinks back below $d$, before being severed at $d$. As we observed in the one-crossover scenario, if a microtubule arrives at $2 d$ it can be severed there in two distinct ways: either it is a microtubule that grows indefinitely and hence is severed with probability 1 , or the severing at $2 d$ wins the competition against the removal of the same crossover due to dynamic instability. In the first case, that accounts a fraction $S_{+}(\infty \mid 0)$ of the microtubules (see Chapter 1 ), a microtubule shrinks back below $d$ if $\tau_{d}>T_{d}+\tau_{2 d}+T_{\sigma}(d)$, with associated probability density function

$$
\begin{aligned}
& P_{\tau_{d}-T_{d}-\tau_{2 d}-T_{\sigma}(d)}(t) \\
& \quad=\int_{\mathbb{R}^{3}} d t^{\prime} d t^{\prime \prime} d t^{\prime \prime \prime} W_{k \theta}\left(t+t^{\prime}+t^{\prime \prime}+t^{\prime \prime \prime}\right) F_{0 d}\left(t^{\prime}\right) W_{k \theta}\left(t^{\prime \prime}\right) L_{\sigma}\left(t^{\prime \prime \prime} \mid d\right)
\end{aligned}
$$

where we used again the relation (3.24), while in the second case, that accounts the remaining $1-S_{+}(\infty \mid 0)$, the microtubule shrinks below without being severed there if $\tau_{d}>T_{d}+\widetilde{\tau}_{2 d}+T_{\sigma}(d)$, and simultaneously if it is severed at $2 d$, i.e. if $\tau_{d}<T_{+}(0)$. The probability density function associated with the first condition is

$$
\begin{aligned}
& P_{\tau_{d}-T_{d}-\widetilde{\tau}_{2 d}-T_{\sigma}(d)}(t) \\
& \quad=\int_{\mathbb{R}^{3}} d t^{\prime} d t^{\prime \prime} d t^{\prime \prime \prime} \widetilde{W}_{k \theta}\left(t+t^{\prime}+t^{\prime \prime}+t^{\prime \prime \prime}\right) F_{0 d}\left(t^{\prime}\right) W_{k \theta}\left(t^{\prime \prime}\right) L_{\sigma}\left(t^{\prime \prime \prime} \mid d\right),
\end{aligned}
$$

while for the second condition is expressed in Eq. (3.25). Therefore, the final expression for $q_{s e v, \sigma}$ is

$$
\begin{aligned}
q_{\text {sev }, \sigma}=R_{d}^{+}(0) & \left\{S_{+}(\infty \mid 0) \int_{0}^{\infty} d t P_{\tau_{d}-T_{d}-\tau_{2 d}-T_{\sigma}(d)}(t)\right. \\
& \left.+\left[1-S_{+}(\infty \mid 0)\right] \int_{0}^{\infty} d t P_{\tau_{d}-T_{0}}(t) \int_{0}^{\infty} d t P_{\tau_{d}-T_{d}-\widetilde{\tau}_{2 d}-T_{\sigma}(d)}(t)\right\} \\
\times & {\left[1-S_{+}(\infty \mid d)\right], }
\end{aligned}
$$

where the first factor $R_{d}^{+}(0)$ accounts all microtubules that reach length $2 d$ starting from $d$, and the final factor $1-S_{+}(\infty \mid d)$ accounts all microtubules that after the severing at $2 d$ shrink back below $d$.

To calculate $q_{n s}$ we first define the random variable $\widetilde{T}_{+}$as the time that a microtubule starting in the growing state and with plus end at $2 d$ needs in order to return in the shrinking state at $2 d$, with the further condition that no severing event 
occurs at $2 d$. Similarly to the derivation of $\widetilde{W}_{k, \theta}(t)$, we can derive the probability density function of $\widetilde{T}_{+}$, that is

$$
\widetilde{L}_{+}(t)=\frac{1}{Z_{L}} L_{+}(t \mid 0) \int_{0}^{t} d t^{\prime} W_{k, \theta}\left(t^{\prime}\right)
$$

with

$$
Z_{L}=\int_{0}^{\infty} d t L_{+}(t \mid 0) \int_{0}^{t} d t^{\prime} W_{k, \theta}\left(t^{\prime}\right)
$$

Therefore, as $q_{n s}$ is basically the probability that a microtubule reaches length $2 d$ and would return to length $d$ but it cannot because it is severed at $2 d$, the two conditions that our random variables have to fullfil are $\tau_{2 d}<T_{+}(0)$ and $\tau_{d}>T_{d}+\widetilde{T}_{+}+T_{-}(d)$. The former condition has already been discussed before, whilst the latter has associated probability density function

$$
\begin{aligned}
& P_{\tau_{d}-T_{d}-\widetilde{T}_{+}-T_{-}(d)}(t) \\
& \quad=\int_{\mathbb{R}^{3}} d t^{\prime} d t^{\prime \prime} d t^{\prime \prime \prime} W_{k \theta}\left(t+t^{\prime}+t^{\prime \prime}+t^{\prime \prime \prime}\right) F_{0 d}\left(t^{\prime}\right) \widetilde{L}_{+}\left(t^{\prime \prime}\right) L_{-}\left(t^{\prime \prime \prime} \mid d\right) .
\end{aligned}
$$

Therefore

$$
q_{n s}=R_{0 d}^{+}\left[1-S_{+}(\infty \mid 0)\right] \int_{-\infty}^{0} d t P_{\tau_{d}-T_{+}(0)}(t) \int_{0}^{\infty} d t P_{\tau_{d}-T_{d}-\widetilde{T}_{+}-T_{-}(d)}(t) .
$$

Finally, in order to calculate the probability $p_{2 d}$ to have a severing event at $2 d$ before an eventual severing event at $d$, we notice that we have two different cases. In the first case, the microtubule reaches length $2 d$ and is severed there before being severed at $d$, i.e. $\tau_{d}>T_{d}+\tau_{2 d}$. In the second case, the microtubule reaches $2 d$ and it is severed there before being severed at $d$, i.e. $\tau_{d}>T_{d}+\widetilde{\tau}_{2 d}$, with the further condition that the event "severing" wins the competition against the event "shrinkage" at $2 d$, or in other instances $\tau_{2 d}<T_{+}(0)$. The associate probability density functions to these conditions are, respectively,

$$
\begin{gathered}
P_{\tau_{d}-T_{d}-\tau_{2 d}}(t)=\int_{\mathbb{R}^{2}} d t^{\prime} d t^{\prime \prime} W_{k \theta}\left(t+t^{\prime}+t^{\prime \prime}\right) F_{0 d}\left(t^{\prime}\right) W_{k \theta}\left(t^{\prime \prime}\right), \\
P_{\tau_{d}-T_{d}-\widetilde{\tau}_{2 d}}(t)=\int_{\mathbb{R}^{2}} d t^{\prime} d t^{\prime \prime} W_{k \theta}\left(t+t^{\prime}+t^{\prime \prime}\right) F_{0 d}\left(t^{\prime}\right) \widetilde{W}_{k \theta}\left(t^{\prime \prime}\right), \\
P_{\tau_{2 d}-T_{+}(0)}(t)=P_{\tau_{d}-T_{+}(0)}(t)
\end{gathered}
$$


therefore

$$
\begin{aligned}
p_{2 d}=R_{0 d}^{+}\{ & S_{+}(\infty \mid 0) \int_{0}^{\infty} d t P_{\tau_{d}-T_{d}-\tau_{2 d}}(t) \\
& \left.+\left[1-S_{+}(\infty \mid 0)\right] \int_{-\infty}^{0} d t P_{\tau_{2 d}-T_{+}(0)}(t) \int_{0}^{\infty} d t P_{\tau_{d}-T_{d}-\tau_{2 d}}(t)\right\} .
\end{aligned}
$$



CHAPTER 4

\section{Reorientation of the cortical microtubule array in presence of finite tubulin pool}

The experiments we presented in Chapter 2 showed that in presence of a high probability of rescue after severing and a stable minus end, cortical microtubules could reorient and maintain the new orientation for a relatively long period of time, see Figure 2.5. Indeed, one could observe that, in around 30 minutes time, the transverse array completely disappeared in advantage of a new longitudinal population of microtubules. However, the model we computationally and analytically studied in Chapters 2 and 3, only looked at the first stage of this reorientation process, i.e. the first 500 seconds after the exposure to light. In that time frame, the transverse array could be considered as a constant background, and indeed we assumed the dynamics only for longitudinal microtubules.

Here, we want to study the behaviour of the system during the long-time phase of reorientation process. In particular, we are interested in understanding which factors play a role in the success and in the maintenance of the new longitudinal array of microtubules. Experimental observations have already shown the existence of a preferential severing for the more recently nucleated microtubules rather than the older ones. As in the initial phase of the process all background microtubules are transverse, there is a strong correlation between new and longitudinal microtubules, resulting in a preferential severing mechanism for the latter. In this chapter, we test the hypothesis whether the asymmetry induced by the preferential severing 
can explain the reorientation, and maintain the new longitudinal array.

In this chapter, we will introduce a theoretical model of two populations of microtubules - transverse and longitudinal, that compete for a finite tubulin pool, and interact with each other to create new microtubules through severing, starting from the initial transverse array. In Section 4.1 we study the model with the asymmetry induced by the preferential severing for longitudinal microtubules. Although this model explains the fast initial amplification of the new longitudinal array, does not give a good explanation of the long term maintained reorientation. Nevertheless, it provides us interesting observations for a change in the model features that could explain the observed phenomena. Therefore, in Section 4.2 we study the same model without severing events, but with another asymmetry induced by a small difference in the growth speeds of the two populations of microtubules. In this way, we show that the advantage coming from a slightly higher growing speed can completely reorient the array from a transverse to a longitudinal direction, and can maintain the array in the new orientation. Finally, in Section 4.3, we computationally test the model with both different speeds for growing microtubules belonging to different populations and interaction between them due to severing. We show that the combination of the two effects not only explains the full reorientation and the maintenance of the array, but also considerably shortens the time scale of the process.

\subsection{Preferential severing of longitudinal microtubules does not explain a maintained reorientation}

\section{The model}

The model, based on the Dogterom-Leibler model, consists of two populations of microtubules undergoing dynamic instability: longitudinal $\left(M_{\|}\right)$and transverse $\left(M_{\perp}\right)$. The two populations compete for a finite tubulin pool, i.e. they have a finite amount of building material that they can use to grow and to be nucleated. If $L_{t o t}$ is the total amount of tubulin in the system, it follows that it is divided in three different classes: free tubulin pool $L_{f}$, tubulin used by longitudinal microtubules $L_{\|}$, and tubulin used by transverse microtubules $L_{\perp}$, such that

$$
L_{t o t}=L_{f}+L_{\|}+L_{\perp}
$$

In order to take into account the finiteness of the tubulin pool, we model the growth speed of microtubules with a saturating chemical equilibrium, that takes 
into account the fact that the more free tubulin is present in the pool, the faster is the growth of microtubules. Practically, this implies the introduction of a constant $L_{v}$ that is expression of finiteness of the pool and diffusion of the free tubulin (Cytrynbaum et al., J. Cell Sci., 2004). Therefore, the expression for the speed of microtubules in the growing state is

$$
V^{+}\left(L_{f}\right)=v^{+} \frac{L_{f}}{L_{f}+L_{v}} .
$$

Microtubules in the shrinking state shrink with speed $v^{-}$, and they can switch from the growing to the shrinking state and viceversa with catastrophe rate $r_{c}$ and rescue rate $r_{r}$, respectively.

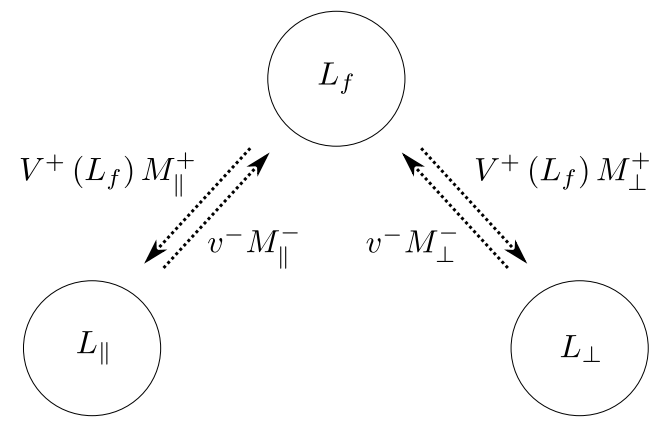

Figure 4.1: Tubulin redistribution in the system. Tubulin leaves the free pool to build microtubules of the two dynamic population with a rate proportional to the total number of growing microtubules, while it goes back in the free pool with a rate proportional to the total number of shrinking microtubules.

The two populations of microtubules interact with each other through severing events at crossovers. As in this chapter we are interested more in explaining how with the severing mechanism it is possible to maintain the reoriented array, rather than in the amplification mechanism per se, for the sake of simplicity we assume that severing events can occur over the whole length of a microtubule, with a severing rate proportional to the latter. Nevertheless, since in cells a microtubule is severed depending on how many crossovers it creates with differently oriented microtubules, here we define a severing rate that takes that into account. To that end, we assume that all cortical microtubules live on the lateral surface of a cylinder of height $h$ and radius $r$. To fix the ideas, let $l$ be the length of a single longitudinal microtubule. Then, the probability for that microtubule to create a crossover with a transverse one is

$$
p_{\text {cross }}(l)=\frac{l}{h} \sum_{i=1}^{M_{\perp}} \frac{l_{\perp, i}}{2 \pi r},
$$


where $l_{\perp, i}$ is the length of the $i$ th transverse microtubule. Then

$$
p_{\text {cross }}(l)=\frac{1}{2 \pi r h} l L_{\perp} .
$$

Furthermore, as we said in the introduction of the chapter, to be consistent with the experimental observations, we implement a preferential severing for the newer (longitudinal) microtubules (Lindeboom et al., Science, 2013). In order to do so, we introduce the probability of severing a longitudinal microtubule $q>\frac{1}{2}$. Then, if $r_{s}$ is the intrinsic severing rate at a crossover, the overall severing rate for a longitudinal or transverse microtubule of length $l$ is

$$
R_{s, \|}(l)=q r_{s} l L_{\perp},
$$

or

$$
R_{s, \perp}(l)=(1-q) r_{s} l L_{\|},
$$

respectively, and where the factor $\frac{1}{2 \pi r h}$ has been incorporated in the severing rate $r_{s}$. Accordingly to the previous chapters, once a microtubule is severed, the newly-created plus end of the lagging microtubule enters either the growing state with probability $p^{+}$, or the shrinking state with probability $1-p^{+}$, while no changes are applied to the state of the plus end of the leading microtubule. Finally, the newly-created minus end of the leading microtubule stays stable, without any dynamics.

The last ingredient of the model is the nucleation of new microtubules. Besides the creation of new microtubules by severing, consistently with in vivo observations (Ehrhardt, Curr. Opin. Cell Biol., 2008), here we assume that microtubules are mainly - but not exclusively, nucleated by branching processes starting from already existing microtubules. In particular, if $R_{n}$ is the overall nucleation rate of the new microtubules in the whole system, we know that because of the finite tubulin pool, $R_{n}$ is a function of $L_{f}$, and specifically

$$
R_{n}\left(L_{f}\right)=r_{n} \frac{L_{f}^{a}}{L_{f}^{a}+L_{v}^{a}},
$$

where here the Hill coefficient $a=6$. The nucleation rate can be further split in branching nucleation, with rate

$$
R_{n, b}=R_{n}\left(L_{f}\right) \frac{L_{\|}+L_{\perp}}{L_{\|}+L_{\perp}+L_{*}},
$$

and nucleation from a dispersed site, with rate

$$
R_{n, u}=R_{n}\left(L_{f}\right) \frac{L_{*}}{L_{\|}+L_{\perp}+L_{*}},
$$


where $L_{*}$ is the propensity length for dispersed nucleation, i.e. a constant that controls how many nucleation events occur from dispersed sites in the cytosol rather than on the lattice of already existing microtubules. These nucleation rates can be split again to divide nucleation in the longitudinal direction from nucleation in the transverse. In the cortical microtubule array, when a new microtubule is nucleated from the lattice of an already existing one through branched nucleation, it is nucleated preferentially parallel or with an angle of about $40^{\circ}$ with respect to the growth direction of the parent microtubule (Deinum et al., Phys. Biol., 2011). This suggests the existence of a nucleation mechanism that roughly maintains the orientation of the microtubule array. As here we only consider two possible directions for microtubules, we assume that new microtubules generated through branched nucleation have a strong bias towards growing in the same direction as the parent microtubule. Therefore, we introduce the probability $z$ for a new microtubule to be nucleated parallel to the parent microtubule, and consequently $1-z$ as the probability to be nucleated orthogonal to it. Then, the branching nucleation rates for new longitudinal and transverse microtubules are

$$
R_{n, b}^{\|}=R_{n}\left(L_{f}\right) \frac{z L_{\|}+(1-z) L_{\perp}}{L_{\|}+L_{\perp}+L_{*}}
$$

and

$$
R_{n, b}^{\perp}=R_{n}\left(L_{f}\right) \frac{(1-z) L_{\|}+z L_{\perp}}{L_{\|}+L_{\perp}+L_{*}}
$$

respectively. If we assume that the medium is isotropic as regards the dispersed nucleation of new microtubules, we can also write the dispersed nucleation rates as

$$
R_{n, u}^{\|}=R_{n}\left(L_{f}\right) \frac{\frac{1}{2} L_{*}}{L_{\|}+L_{\perp}+L_{*}}=R_{n, u}^{\perp} .
$$

It follows that the overall nucleation rates for the two populations are

$$
\begin{aligned}
& R_{n}^{\|}=R_{n, u}^{\|}+R_{n, b}^{\|}=R_{n}\left(L_{f}\right) \frac{\frac{1}{2} L_{*}+z L_{\|}+(1-z) L_{\perp}}{L_{\|}+L_{\perp}+L_{*}}, \\
& R_{n}^{\perp}=R_{n, u}^{\perp}+R_{n, b}^{\perp}=R_{n}\left(L_{f}\right) \frac{\frac{1}{2} L_{*}+z L_{\perp}+(1-z) L_{\|}}{L_{\|}+L_{\perp}+L_{*}},
\end{aligned}
$$

see Figure 4.2.

Numerical values for the model parameters have been chosen accordingly to the experimental measurements already discussed in Chapter 2, see Table 4.1. As regards the numerical value for the severing rate, is has been chosen considering a 


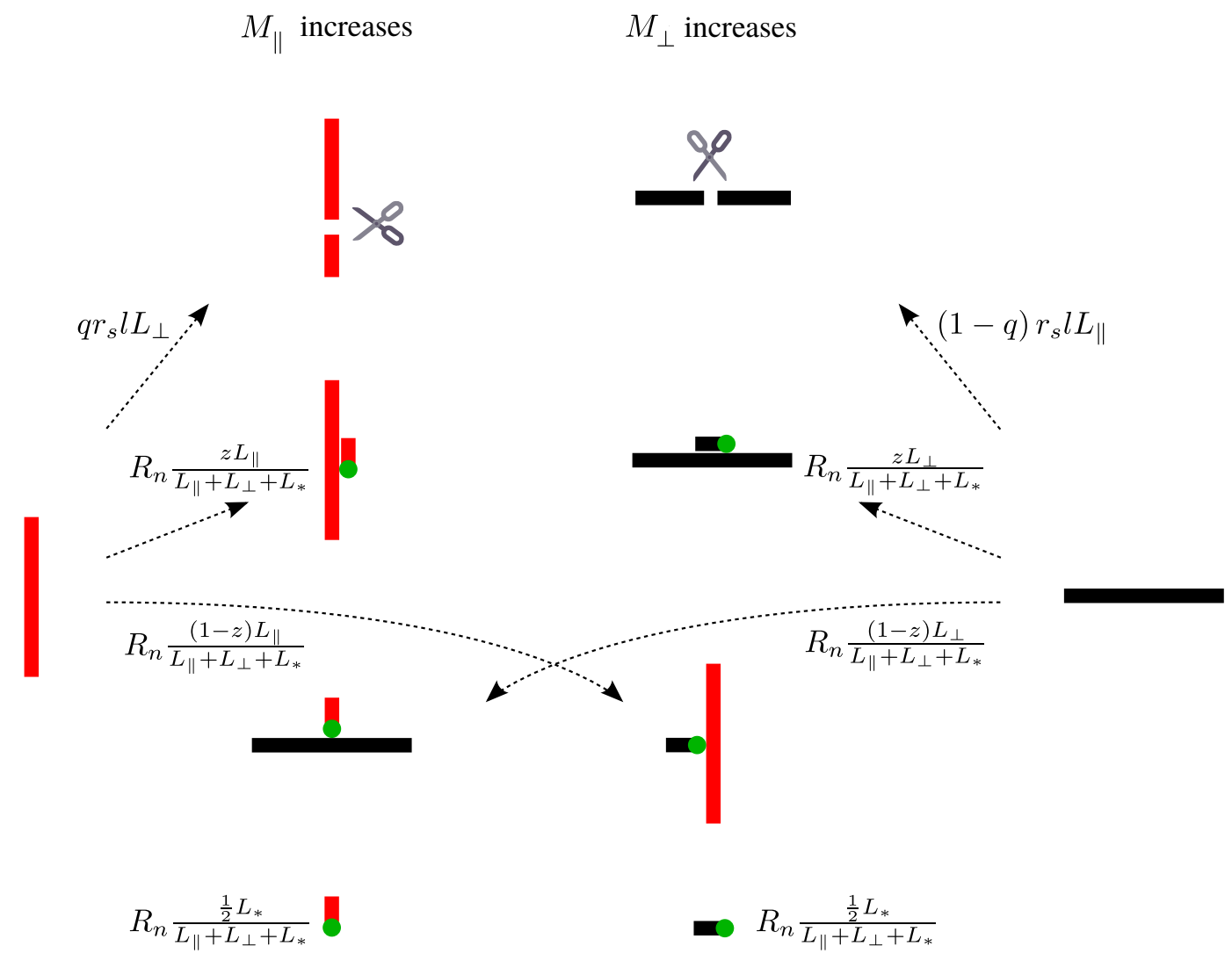

Figure 4.2: Schematic of the possible creation of new longitudinal (red) and transverse (black) microtubules. 
Reorientation of the cortical microtubule array in presence of finite tubulin pool

\begin{tabular}{|cccc|}
\hline Parameter & Description & Numerical value & Units \\
\hline$v^{+}$ & Growth speed & 0.103 & $\mu \mathrm{m} \mathrm{s}^{-1}$ \\
$v^{-}$ & Shrinkage speed & 0.225 & $\mu \mathrm{m} \mathrm{s}^{-1}$ \\
$r_{c}$ & Catastrophe rate & 0.01 & $\mathrm{~s}^{-1}$ \\
$r_{r}$ & Rescue rate & 0.026 & $\mathrm{~s}^{-1}$ \\
$r_{s}$ & Severing rate & $2 \cdot 10^{-7}$ & $\mathrm{~s}^{-1} \mu \mathrm{m}^{-2}$ \\
$r_{n}$ & Nucleation rate & 0.3 & $\mathrm{~s}^{-1}$ \\
$p^{+}$ & Rescue after severing & 0.15 & - \\
$q$ & Sever probability (longitudinal microtubules) & 0.75 & - \\
$L_{t o t}$ & Total amount of tubulin & $4 \cdot 10^{3}$ & $\mu \mathrm{m}$ \\
$L_{v}$ & Crossover length & $8 \cdot 10^{2}$ & $\mu \mathrm{m}$ \\
$L_{*}$ & Propensity length of dispersed nucleation & $10^{2}$ & - \\
$z$ & Probability of nucleation parallel to the parent & 0.9 & \\
\hline
\end{tabular}

Table 4.1: Reference values for the parameters of the model.

cylindrical cell with height $h=100 \mu \mathrm{m}$ and radius $r=20 \mu \mathrm{m}$, and such that the average waiting time between the nucleation of a new microtubule and the first severing event it undergoes is about 100 seconds. Even though the probability of rescue after severing $p^{+}$and the probability of severing for longitudinal microtubules $q$ will be tuned in the rest of the chapter, Table 4.1 gives their reference values from the experimental measurements (Lindeboom et al., Science, 2013).

\section{Dynamic equations}

Let $m^{\sigma}(t, l)$ be the probability distribution of the length of microtubules in the state $\sigma$ at time $t$. Besides the in and out-flux of microtubules at a length $l$ due to the dynamic instability, here we also have to take into account the fluxes due to the severing events. Therefore, following (Tindemans \& Mulder, Phys. Rev. E, 2010), we can show that the dynamic equations that govern the model are

$$
\begin{aligned}
\frac{\partial}{\partial t} m_{\|}^{+}(t, l)= & -V^{+}\left(L_{f}\right) \frac{\partial}{\partial l} m_{\|}^{+}(t, l)-r_{c} m_{\|}^{+}(t, l)+r_{r} m_{\|}^{-}(t, l) \\
& -q r_{s} l L_{\perp} m_{\|}^{+}(t, l)+q r_{s}\left(1+p^{+}\right) L_{\perp}\left[M_{\|}^{+}(t, l)+M_{\|}^{-}(t, l)\right] \\
& +q r_{s} L_{\perp} M_{\|}^{+}(t, l),
\end{aligned}
$$




$$
\begin{aligned}
& \frac{\partial}{\partial t} m_{\|}^{-}(t, l)=v^{-} \frac{\partial}{\partial l} m_{\|}^{-}(t, l)-r_{r} m_{\|}^{-}(t, l)+r_{c} m_{\|}^{+}(t, l) \\
& -q r_{s} l L_{\perp} m_{\|}^{-}(t, l)+q r_{s}\left(1-p^{+}\right) L_{\perp}\left[M_{\|}^{+}(t, l)+M_{\|}^{-}(t, l)\right] \\
& +q r_{s} L_{\perp} M_{\|}^{-}(t, l) \text {, } \\
& \frac{\partial}{\partial t} m_{\perp}^{+}(t, l)=-V^{+}\left(L_{f}\right) \frac{\partial}{\partial l} m_{\perp}^{+}(t, l)-r_{c} m_{\perp}^{+}(t, l)+r_{r} m_{\perp}^{-}(t, l) \\
& -(1-q) r_{s} l L_{\|} m_{\perp}^{+}(t, l) \\
& +(1-q) r_{s}\left(1+p^{+}\right) L_{\|}\left[M_{\perp}^{+}(t, l)+M_{\perp}^{-}(t, l)\right] \\
& +(1-q) r_{s} L_{\|} M_{\perp}^{+}(t, l) \text {, } \\
& \frac{\partial}{\partial t} m_{\perp}^{-}(t, l)=v^{-} \frac{\partial}{\partial l} m_{\perp}^{-}(t, l)-r_{r} m_{\perp}^{-}(t, l)+r_{c} m_{\perp}^{+}(t, l) \\
& -(1-q) r_{s} l L_{\|} m_{\perp}^{-}(t, l) \\
& +(1-q) r_{s}\left(1-p^{+}\right) L_{\|}\left[M_{\perp}^{+}(t, l)+M_{\perp}^{-}(t, l)\right] \\
& +(1-q) r_{s} L_{\|} M_{\perp}^{-}(t, l),
\end{aligned}
$$

with boundary conditions

$$
\begin{gathered}
V^{+}\left(L_{f}\right) m_{\| / \perp}^{+}(t, 0)=R_{n}^{\| / \perp}\left(L_{f}, L_{\| / \perp}\right), \\
\lim _{l \rightarrow \infty} m_{\| / \perp}^{-}(t, l)=0 .
\end{gathered}
$$

It is convenient to underline that the total amount of tubulin polarized in either of the two directions is linked with the microtubule length distributions as

$$
\begin{aligned}
& L_{\|}(t)=\int_{0}^{\infty} d l l\left[m_{\|}^{+}(t, l)+m_{\|}^{-}(t, l)\right], \\
& L_{\perp}(t)=\int_{0}^{\infty} d l l\left[m_{\perp}^{+}(t, l)+m_{\perp}^{-}(t, l)\right],
\end{aligned}
$$

i.e. the total amount of tubulin used by longitudinal/transverse microtubules is the first moment of the total length distribution of longitudinal/transverse microtubules.

\section{The initial transverse array}

To keep with the assumption coming from the experiments that initially all cortical microtubules are directed transversely to the growth direction of the cell, we build 
the initial array by considering dispersed and branching nucleation possible only in the transverse direction. In other words, for the creation of the initial array, we impose

$$
\begin{gathered}
R_{n}^{\|}=0, \\
R_{n}^{\perp}=r_{n} \frac{L_{f}^{a}}{L_{f}^{a}+L_{v}^{a}}=R_{n}\left(L_{f}\right) .
\end{gathered}
$$

With these conditions, Eqs. (4.13) and (4.14) reduce to the dynamic equations of the Dogterom-Leibler model. Furthermore, the dependency of the growing speed of microtubules on the amount of free tubulin $L_{f}$ in the pool implies that the microtubule length distribution eventually reaches the steady-state even in the case of initially unbounded-growth microtubules (Tindemans, 2009). Thus, the solution of the model is the steady-state solution of Dogterom-Leibler model, and we use this solution as the initial condition for the system of Eqs. (4.11-4.14), i.e.

$$
\begin{gathered}
m_{\|}^{+}(0, l)=0, \\
m_{\|}^{-}(0, l)=0, \\
m_{\perp}^{+}(0, l)=\frac{R_{n}\left(L_{f}\right)}{V^{+}\left(L_{f}\right)} \exp \left[-\left(\frac{r_{c}}{V^{+}\left(L_{f}\right)}-\frac{r_{r}}{v^{-}}\right) l\right], \\
m_{\perp}^{-}(0, l)=\frac{R_{n}\left(L_{f}\right)}{v^{-}} \exp \left[-\left(\frac{r_{c}}{V^{+}\left(L_{f}\right)}-\frac{r_{r}}{v^{-}}\right) l\right],
\end{gathered}
$$

together with the Eq. (4.1) for the free tubulin pool. As in the rest of the chapter we are going to study the system in the limit of sudden depolymerization after catastrophe, it is convenient to briefly discuss the expression for the free tubulin in such a limit, i.e.

$$
L_{f}^{2}-\left(L_{t o t}-L_{v}-\frac{v^{+} R_{n}\left(L_{f}\right)}{r_{c}^{2}}\right) L_{f}-L_{t o t} L_{v}=0 .
$$

This is a high order algebraic equation. However, the coefficient $L_{t o t}-L_{v}-\frac{v^{+} R_{n}\left(L_{f}\right)}{r_{c}^{2}}$ is dominated by the factor $L_{t o t}-L_{v}$, see Table 4.1 , and consequently changing any of the free parameters in $\frac{v^{+} R_{n}\left(L_{f}\right)}{r_{c}^{2}}$ within a realistic biological range would only give minor changes to the solution $L_{f} \simeq L_{t o t}$. Still, the limit of sudden depolymerization allows us to achieve interesting analytically results, that with the full model would be either non-achievable or with very complicated and non-handy mathematical expressions. 


\section{Steady-state solution}

The dependency of the growing speed of microtubules on the amount of free tubulin $L_{f}$ in the pool implies that the microtubule length distribution eventually reaches the steady-state. Here, in order to find an analytical solution, we make the further assumption that microtubules undergo complete depolymerization suddenly after a catastrophe, i.e.

$$
\frac{\langle l\rangle}{v^{-}} \ll \frac{1}{r_{r}},
$$

where $\langle l\rangle$ is the mean length of a microtubule in the system. In this limit we can identify all microtubules with the growing microtubules, and then we remove the label + from all microtubule distributions to ease the notation. To further ease the notation, if not strictly necessary, we hereafter drop the direct dependency of $V^{+}$and $R_{n}$ on $L_{f}$. Thus, Eqs. (4.11-4.14) are replaced by

$$
\begin{gathered}
0=-V^{+} \frac{d}{d l} m_{\|}(l)-r_{c} m_{\|}(l)-q r_{s} l L_{\perp} m_{\|}(l)+q r_{s}\left(1+p^{+}\right) L_{\perp} M_{\|}(l), \\
0=-V^{+} \frac{d}{d l} m_{\perp}(l)-r_{c} m_{\perp}(l)-(1-q) r_{s} l L_{\|} m_{\perp}(l) \\
+(1-q) r_{s}\left(1+p^{+}\right) L_{\|} M_{\perp}(l) .
\end{gathered}
$$

If we differentiate by $l$ the last two equations we obtain

$$
\begin{gathered}
V^{+} \frac{d^{2}}{d l^{2}} m_{\|}(l)+\left(r_{c}+q r_{s} L_{\perp} l\right) \frac{d}{d l} m_{\|}(l)+q r_{s} L_{\perp}\left(2+p^{+}\right) m_{\|}(l)=0, \quad(4.25) \\
V^{+} \frac{d^{2}}{d l^{2}} m_{\perp}(l)+\left(r_{c}+(1-q) r_{s} L_{\|} l\right) \frac{d}{d l} m_{\perp}(l)+(1-q) r_{s} L_{\|}\left(2+p^{+}\right) m_{\perp}(l)=0 .
\end{gathered}
$$

As $L_{\| / \perp}=\int_{0}^{\infty} d l m_{\| / \perp}(l)$, Eqs. (4.25) and (4.26) are coupled second order differential equations, and they are also linked to Eq. (4.1) through $V^{+}\left(L_{f}\right)$. However, $L_{f}$, $L_{\|}$, and $L_{\perp}$ do not depend on $l$, hence Eqs. (4.25) and (4.26) can be in principle solved, with solutions that depend on the total amount of tubulin used by the other population and on the free tubulin. Before solving them, we re-write the two equations in a more elegant way as

$$
\begin{gathered}
\frac{d^{2}}{d \lambda^{2}} \mu_{\|}(\lambda)+\left(1+q \sigma \Lambda_{\perp} \lambda\right) \frac{d}{d \lambda} \mu_{\|}(\lambda)+q \sigma \Lambda_{\perp}\left(2+p^{+}\right) \mu_{\|}(\lambda)=0, \\
\frac{d^{2}}{d \lambda^{2}} \mu_{\perp}(\lambda)+\left(1+(1-q) \sigma \Lambda_{\|} \lambda\right) \frac{d}{d \lambda} \mu_{\perp}(\lambda)+(1-q) \sigma \Lambda_{\|}\left(2+p^{+}\right) \mu_{\perp}(\lambda)=0,
\end{gathered}
$$


where we introduced the non-dimensional quantities

$$
\begin{gathered}
\lambda=\frac{r_{c}}{V^{+}} l, \\
\Lambda_{\| / \perp}=\frac{r_{c}}{V^{+}} L_{\| / \perp}, \\
\mu_{\| / \perp}=\frac{V^{+}}{R_{n}^{\| / \perp}} m_{\| / \perp}, \\
\sigma=\frac{r_{s}\left(V^{+}\right)^{2}}{r_{c}^{3}} .
\end{gathered}
$$

Notice that, with this non-dimensionalization, all parameters of the model - including the independent variable $\lambda$, are functions of $L_{f}$ as they depend on $V^{+}\left(L_{f}\right)$.

To solve Eq. (4.27), we first notice that the asymptotic solution for large $\lambda$ decays as $e^{-\frac{1}{2} q \sigma \Lambda_{\perp} \lambda^{2}-\lambda}$. Therefore, we suppose there exists a function $\xi_{\|}(\lambda)$ such that

$$
\mu_{\|}(\lambda)=\exp \left[-\frac{1}{2} q \sigma \Lambda_{\perp} \lambda^{2}-\lambda\right] \xi_{\|}(\lambda)
$$

If we plug this in Eq. (4.27) we obtain

$$
\frac{d^{2}}{d \lambda^{2}} \xi_{\|}(\lambda)-\left(1+q \sigma \Lambda_{\perp} \lambda\right) \frac{d}{d \lambda} \xi_{\|}(\lambda)+q \sigma \Lambda_{\perp}\left(1+p^{+}\right) \xi_{\|}(\lambda)
$$

If we change variable as $x=\left(1+q \sigma \Lambda_{\perp} \lambda\right) / \sqrt{2 q \sigma \Lambda_{\perp}}$, last equation becomes

$$
\frac{d^{2}}{d x^{2}} \xi_{\|}(x)-2 x \frac{d}{d x} \xi_{\|}(x)+2\left(1+p^{+}\right) \xi_{\|}(x)
$$

i.e. the Hermite equation (Courant \& Hilbert, 1953), the solution of which is the Hermite function

$$
\xi_{\|}(x)=H_{1+p^{+}}(x) .
$$

Therefore, the full solution for $\mu_{\|}$becomes (Mulder, unpublished)

$$
\mu_{\|}(\lambda)=\frac{H_{1+p^{+}}\left(\left(1+q \sigma \Lambda_{\perp} \lambda\right) / \sqrt{2 q \sigma \Lambda_{\perp}}\right)}{H_{1+p^{+}}\left(1 / \sqrt{2 q \sigma \Lambda_{\perp}}\right)} \exp \left[-\frac{1}{2} q \sigma \Lambda_{\perp} \lambda^{2}-\lambda\right],
$$

and similarly

$$
\begin{gathered}
\mu_{\perp}(\lambda)=\frac{H_{1+p^{+}}\left(\left(1+(1-q) \sigma \Lambda_{\|} \lambda\right) / \sqrt{2(1-q) \sigma \Lambda_{\|}}\right)}{H_{1+p^{+}}\left(1 / \sqrt{2(1-q) \sigma \Lambda_{\|}}\right)} \\
\times \exp \left[-\frac{1}{2}(1-q) \sigma \Lambda_{\|} \lambda^{2}-\lambda\right] .
\end{gathered}
$$


Eqs. (4.32) and (4.33) highlight a peculiar property of the model. Indeed, if $p^{+} \neq 0$, both denominators of the two distribution can be identically 0 . In other words, there exist two values of $\sigma$ such that $H_{1+p^{+}}\left(1 / \sqrt{2 q \sigma \Lambda_{\perp}}\right)=0$ or $H_{1+p^{+}}\left(1 / \sqrt{2(1-q) \sigma \Lambda_{\|}}\right)=0$, with the consequence that the number of longitudinal/transverse microtubules diverges. In the analytically tractable $p^{+}=1$ case these values are $\sigma=\frac{1}{q \Lambda_{\perp}}$ for the divergence of the longitudinal, and $\sigma=\frac{1}{(1-q) \Lambda_{\|}}$ for the divergence of the transverse microtubules.

\section{Moment equations}

We define the $k^{\text {th }}$ moment of the microtubule length distributions as:

$$
\begin{aligned}
& L_{\|}^{(k)}=\int_{0}^{\infty} d l l^{k} m_{\|}(l), \\
& L_{\perp}^{(k)}=\int_{0}^{\infty} d l l^{k} m_{\perp}(l),
\end{aligned}
$$

with $L_{\| / \perp}^{(0)}=M_{\| / \perp}$ and $L_{\| / \perp}^{(1)}=L_{\| / \perp}$. Then, if we multiply Eqs. (4.23) and (4.24) by $l^{k}$ and we integrate them between 0 and $\infty$, we obtain

$$
\begin{aligned}
& 0=\delta_{k, 0} R_{n}^{\|}+k V^{+} L_{\|}^{(k-1)}-r_{c} L_{\|}^{(k)}-q r_{s} \frac{k-p^{+}}{k+1} L_{\perp} L_{\|}^{(k+1)}, \\
& 0=\delta_{k, 0} R_{n}^{\perp}+k V^{+} L_{\perp}^{(k-1)}-r_{c} L_{\perp}^{(k)}-q r_{s} \frac{k-p^{+}}{k+1} L_{\|} L_{\perp}^{(k+1)} .
\end{aligned}
$$

Unfortunately, given that Eqs. (4.34) and (4.35) form an open set of recursive equations, it is impossible to write them in a closed-form for a certain choice of $k$. However, we can highlight some features of the equations by studying $k=0$ and $k=1$ cases. Indeed, we have

$$
\begin{aligned}
& 0=R_{n}^{\|}-r_{c} M_{\|}+q r_{s} p^{+} L_{\perp} L_{\|}, \\
& 0=R_{n}^{\perp}-r_{c} M_{\perp}+q r_{s} p^{+} L_{\|} L_{\perp},
\end{aligned}
$$

and

$$
\begin{aligned}
& 0=V^{+} M_{\|}-r_{c} L_{\|}-q r_{s} \frac{1-p^{+}}{2} L_{\perp} L_{\|}^{(2)} \\
& 0=V^{+} M_{\perp}-r_{c} L_{\perp}-q r_{s} \frac{1-p^{+}}{2} L_{\|} L_{\perp}^{(2)} .
\end{aligned}
$$


Eqs. (4.36) and (4.37) can be rewritten as

$$
\begin{gathered}
M_{\|}=\frac{R_{n}^{\|}}{r_{c}}+q \frac{r_{s}}{r_{c}} p^{+} L_{\|} L_{\perp} \\
M_{\perp}=\frac{R_{n}^{\perp}}{r_{c}}+(1-q) \frac{r_{s}}{r_{c}} p^{+} L_{\|} L_{\perp},
\end{gathered}
$$

showing the intuitive result that the number of microtubules equals the overall creation rate over the catastrophe rate.

Eqs. (4.38) and (4.39) also highlight a peculiar feature: indeed if we look at the $p^{+}=1$ case, we can observe that

$$
\frac{L_{\|}}{M_{\|}}=\frac{V^{+}}{r_{c}}=\frac{L_{\perp}}{M_{\perp}}
$$

i.e. the average length of the microtubules of the two populations is the same and it is independent on both the preferential severing $q$ and on the severing rate $r_{s}$. This is counter-intuitive, indeed for $q>\frac{1}{2}$ not only the longitudinal microtubules are severed more frequently, but also at every severing event their number increases due to $p^{+}=1$, and thus one can argue that the average length of a longitudinal microtubule should be shorter than that of a transverse, in disagreement with Eq. $(4.42)$.

\section{Polarization and transverse suppression}

To evaluate the efficiency of the reorientation, we define the following order parameters:

- number and length polarization, respectively

$$
\mathcal{P}_{M}=\frac{M_{\|}-M_{\perp}}{M_{\|}+M_{\perp}}, \quad \mathcal{P}_{L}=\frac{L_{\|}-L_{\perp}}{L_{\|}+L_{\perp}},
$$

as order parameters for the difference between the two populations,

- number and length transverse suppression, respectively

$$
\mathcal{R}_{M}=\frac{M_{\perp}^{0}-M_{\perp}}{M_{\perp}^{0}+M_{\perp}}, \quad \mathcal{R}_{L}=\frac{L_{\perp}^{0}-L_{\perp}}{L_{\perp}^{0}+L_{\perp}}
$$

as order parameters that estimate how much of the original transverse array is still present at the end of the process.

Ideally, to consider the reorientation efficient, we require that all four order parameters are close to 1 , and that the time scale to reach the steady-state is comparable to the experimentally observed one, see Chapter 2. 


\section{Computational approach}

Since the non-closed-form nature of Eqs. (4.36-4.39) does not allow us to analytically calculate polarization and transverse suppression, we set up stochastic simulations of the full model, i.e. also considering shrinking microtubules and with dynamic parameters chosen in agreement with the experimental measurements, see Table 4.1 .

We want to test our hypothesis that the preferential severing for longitudinal microtubules creates an asymmetry in the system that can make the array reorient and sustain it. To do so, we perform a sensitivity analysis in which we tune $q$ and $p^{+}$from 0 to 1 and we measure, with computer simulations, the polarization and the transverse suppression at the equilibrium, as well as the time needed by the system to perform reorientation of the array, averaged over $N=10^{3}$ simulations.

Simulations consist of an initial transverse array at the equilibrium, created as previously described in this section. Then, at time $t=0$, we allow the nucleation of longitudinal microtubules both through branched nucleation from already existing ones, and dispersed nucleation. We let the simulations run until the system reaches a steady-state, and then we measure polarization and transverse suppression. Figures 4.3AB and 4.4 show that the system does not exhibit longitudinal polarization at the equilibrium for biologically realistic $p^{+}$, i.e. for $p^{+}$comprises between 0 and 0.25 . At the same time, Figure 4.3CD shows that in the same range of values, although we can appreciate some degree of transverse suppression, the initial transverse array does not disappear. On the other hand, Figures 4.3 and 4.4 reveal the existence of two regions in the $\left(q, p^{+}\right)$plane where the reorientation occurs and it is fast, namely when both probabilities are high and, surprisingly, when they are both low. While we can easily argue that a high value of both $q$ and $p^{+}$is associated to a greater likelihood of increasing the size of the longitudinal population and the lifetime of their individuals, and hence the longitudinal polarization, it is more difficult to intuitively understand the behaviour of the system for low values of those probabilities. However, low $q$ together with low $p^{+}$is linked to an effective shortening of the single transverse microtubules, and to a fall in their average lifetime. As a consequence, the overall length used by the transverse array shortens, and so does the number of transverse microtubules, as their nucleation is partly correlated to the length polarized in their direction.

As the only asymmetry between the two populations of the system is due to the probability $q$, Figure 4.3AB displays, as expected, the symmetry $\mathcal{P} \rightarrow-\mathcal{P}$, as $q \rightarrow 1-q$ for both $\mathcal{P}_{M}$ and $\mathcal{P}_{L}$.

Although from these results we can conclude that the preferential severing for the longitudinal microtubules cannot explain the maintenance of the array in the longitudinal direction, at least in the biological range of values for $p^{+}$, the dynamic 

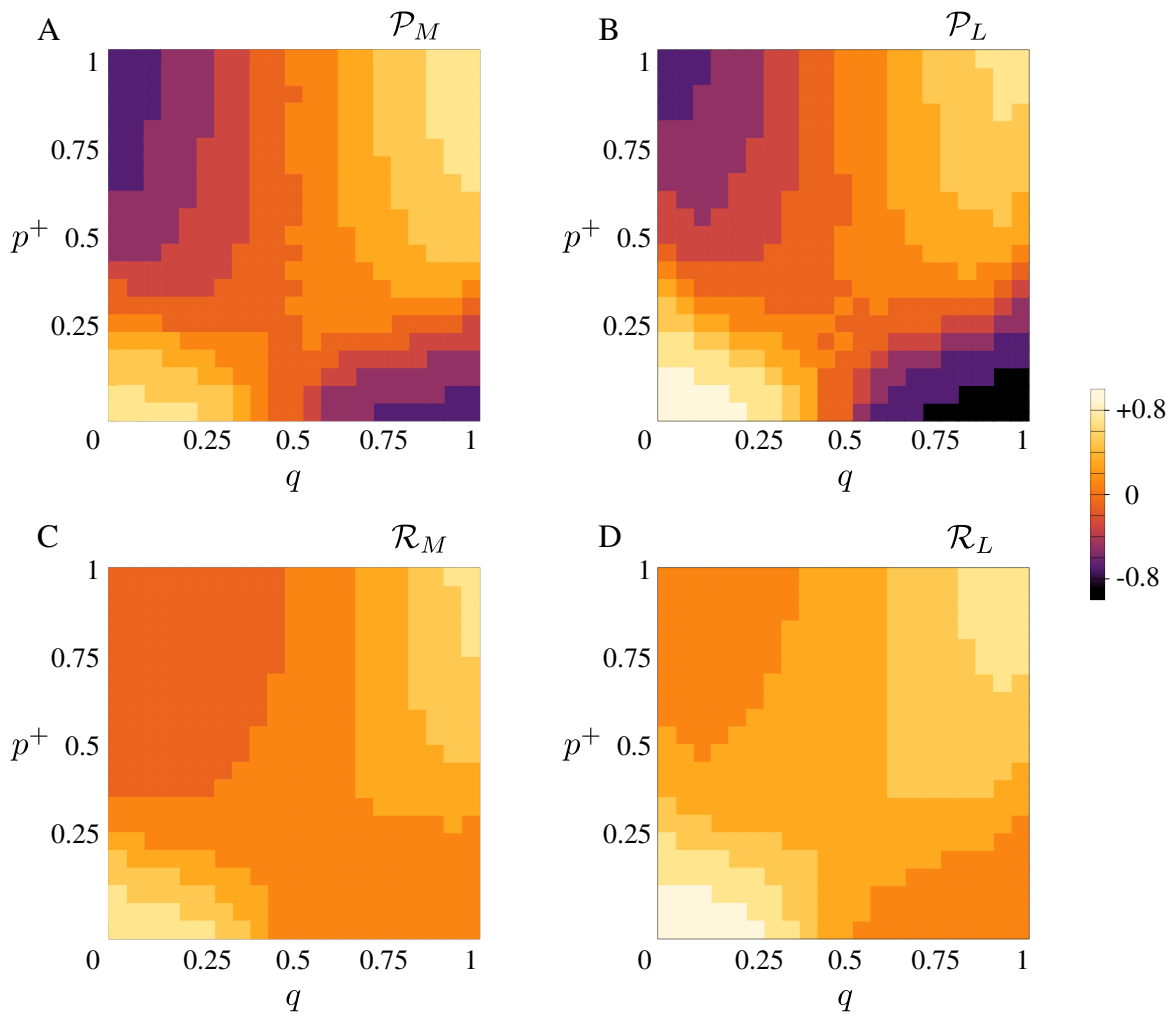

Figure 4.3: (A) Microtubule number polarization, (B) microtubule length polarization, (C) transverse number suppression, (D) and transverse length suppression as functions of $q$ and $p^{+}$. Lighter colors correspond to a more efficient reorientation. Results are averaged over $N=10^{3}$ simulations.

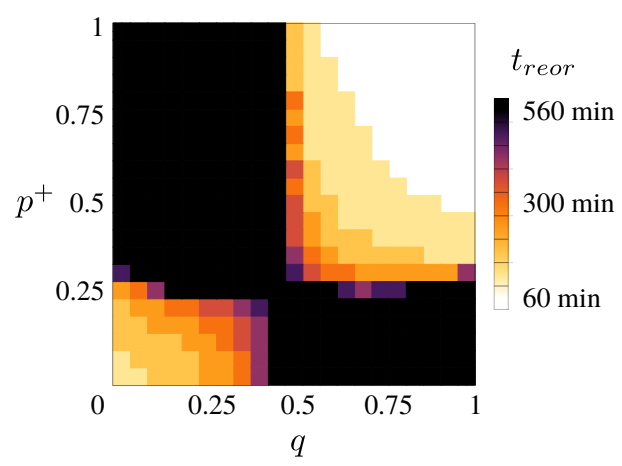

Figure 4.4: Transverse-to-longitudinal reorientation time as a function of $q$ and $p^{+}$. Lighter colors correspond to a faster reorientation. Results are averaged over $N=10^{3}$ simulations. 
behaviour of the two microtubule populations for high values of $q$ and $p^{+}$highlights an interesting fact. The amount of free tubulin in the pool does not substantially change from the initial value where only transverse microtubules are present, to the final steady-state value, see Figure 4.5B. This means that all the building material used by the longitudinal microtubules to create the new array comes from the suppression of the initial transverse array. Curiously, Figure 4.5A also shows that at the start of the reorientation process, i.e. at $t=0$, we have a sudden little drop of the number of transverse microtubules. This may be explained by the sudden change in the nucleation rate of old microtubules, as it switches from $R_{n}$ to a fraction of it.
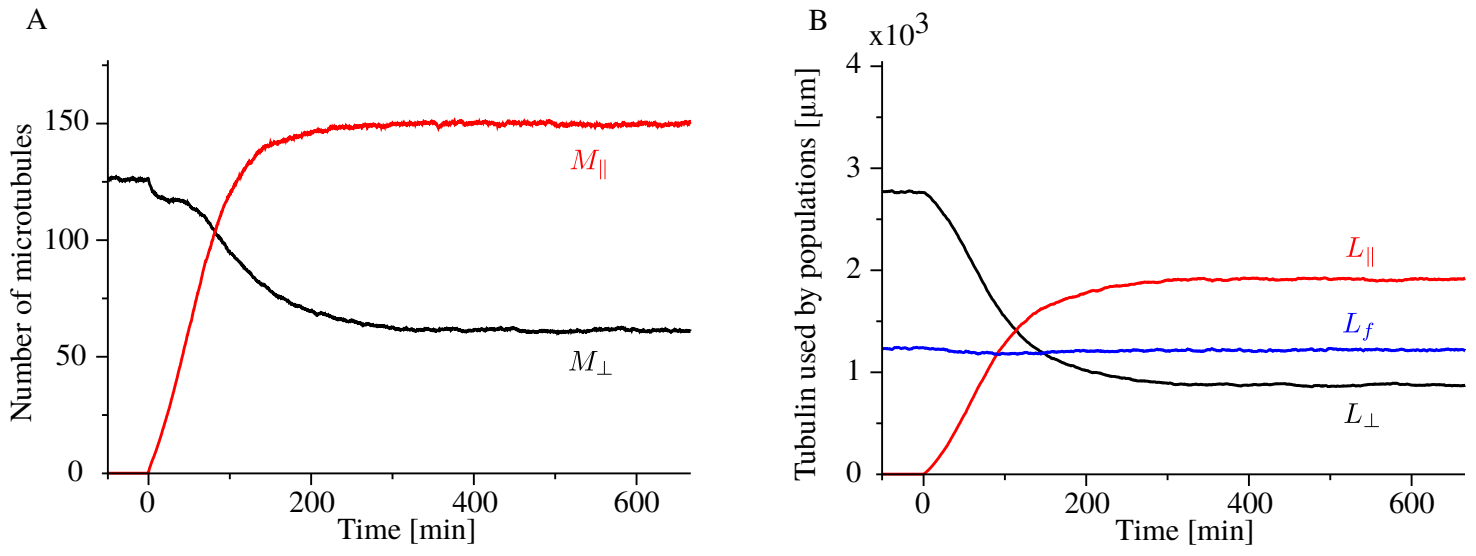

Figure 4.5: Time evolution of (A) longitudinal (red) and transverse (black) microtubules, and (B) tubulin used by the longitudinal population (red), the transverse population (black), and the free tubulin (blue), averaged over $N=10^{3}$ simulations. Here, we used $q=0.8$ and $p^{+}=0.6$.

The results shown so far represent the average behaviour of the system. However, as this model includes stochasticity and it represents two distinct populations interacting with each other, we cannot a priori exclude oscillatory time behaviours in the distribution of populations, with cycles in the $\left(M_{\|}, M_{\perp}\right)$ and $\left(L_{\|}, L_{\perp}\right)$ planes. Indeed it is possible that, for a specific choice of dynamic parameters, two distinct steady-states exist, with the system that can stochastically switch from one state to the other. Here, however, our simulations show that for our choice of dynamic parameters there are no oscillations in the size of the populations, except for the noise coming from the stochasticity of the system. Indeed, Figure 4.6 shows that, except for some stochastic noise, $M_{\|}(t)$ measured in a single simulation reproduces the time behaviour of the same quantity, averaged over $N=10^{3}$ different simulations. Future investigations should be aimed at analytically and 


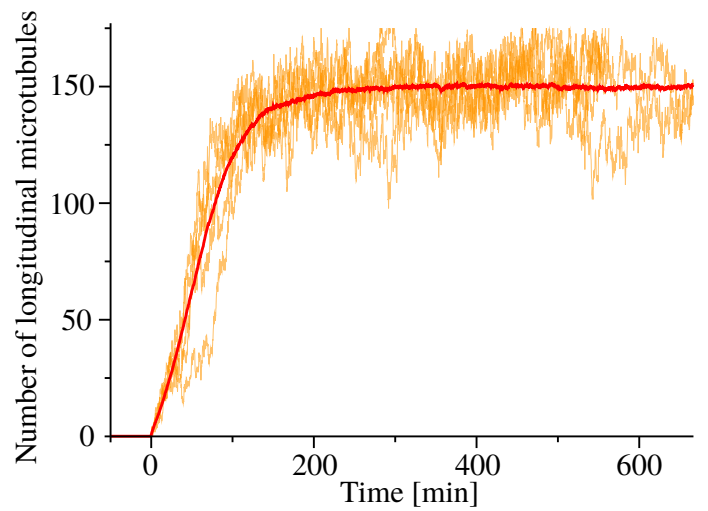

Figure 4.6: Time evolution of $M_{\|}$averaged over $N=10^{3}$ simulations (red line), and in five different single simulations (orange lines). Besides the statistical noise, every single simulation reaches the same steady-state value. Here, we used $q=0.8$ and $p^{+}=0.6$.

computationally checking whether there exists a volume in the parameters space where the two populations evolve in time with an oscillatory behaviour.

\subsection{Small difference in microtubule growth speed maintains the reorientation of the array}

Assuming asymmetry in the severing probability for the two populations of microtubules is not enough to explain the maintenance of the reorientation of the cortical array. Therefore, such a reorientation must be explained by other asymmetries. Here, we make the hypothesis that the final longitudinal polarization is due to a difference in the dynamic parameters of microtubules belonging to the two different populations. Specifically, we assume that longitudinal microtubules grow faster than transverse. This choice is motivated by two distinct reasons. Firstly, experimental observations in neuronal microtubules have shown that differently oriented microtubules living in the same system can have different dynamics (Baas et al., Cytoskeleton, 2016 - Tas et al., Neuron, 2017). Secondly, changing only one parameter - in particular the growing speed, would make the model mathematically more tractable. Moreover, as we will see in the next sections, changing the growing speed is equivalent to changing the catastrophe rate for the study of the model in the steady-state. 


\section{Small increase in the growing speed of longitudinal microtubules}

To test our hypothesis that reorientation and maintenance of the array are caused by a biased recruitment of tubulin towards the longitudinal microtubules, we make use of the model introduced in Section 4.1 with the following changes: no severing events are allowed, i.e.

$$
r_{s}=0
$$

and the growing speed for longitudinal microtubules is greater than that for transverse microtubules, i.e.

$$
v_{\|}^{+}=\alpha v_{\perp}^{+} \equiv \alpha v^{+}, \quad 1<\alpha<2 .
$$

In order to find an analytical solution, we assume once more that the complete depolymerization time after undergoing a catastrophe is much shorter than the average shrinkage time between a catastrophe and a subsequent rescue, i.e.

$$
\frac{\langle l\rangle}{v^{-}} \ll \frac{1}{r_{r}} .
$$

We also assume that all microtubules are nucleated in the same direction as the parent microtubule, i.e. $z=1$. This allows us to rewrite the dynamic Eqs. $(4.11-4.14)$ as

$$
\begin{gathered}
\frac{\partial}{\partial t} m_{\|}(t, l)=-\alpha V^{+} \frac{\partial}{\partial l} m_{\|}(t, l)-r_{c} m_{\|}(t, l), \\
\frac{\partial}{\partial t} m_{\perp}(t, l)=-V^{+} \frac{\partial}{\partial l} m_{\perp}(t, l)-r_{c} m_{\perp}(t, l),
\end{gathered}
$$

with boundary conditions

$$
\begin{gathered}
\alpha V^{+} m_{\|}(t, 0)=R_{n}^{\|}, \\
V^{+} m_{\perp}(t, 0)=R_{n}^{\perp} .
\end{gathered}
$$

\section{Moment equations}

We study the $0^{\text {th }}$ and the $1^{\text {st }}$ moment equations corresponding to Eqs. (4.43) and $(4.44)$, i.e.

$$
\begin{aligned}
\frac{d}{d t} M_{\|}(t) & =R_{n}^{\|}-r_{c} M_{\|}(t), \\
\frac{d}{d t} M_{\perp}(t) & =R_{n}^{\perp}-r_{c} M_{\perp}(t),
\end{aligned}
$$




$$
\begin{aligned}
\frac{d}{d t} L_{\|}(t) & =\alpha V^{+} M_{\|}(t)-r_{c} L_{\|}(t), \\
\frac{d}{d t} L_{\perp}(t) & =V^{+} M_{\perp}(t)-r_{c} L_{\perp}(t),
\end{aligned}
$$

coupled with the conservation of total tubulin

$$
\frac{d}{d t} L_{f}(t)=-\frac{d}{d t}\left[L_{\|}(t)+L_{\perp}(t)\right] .
$$

Suppose now that $\alpha=1$. Then, because of the symmetry, there are no changes in the dynamics of the system with respect to the initial system with only the transverse array. Therefore we can safely assume that in this case $L_{f}=$ const. Here, since $\alpha$ is slightly greater than 1 , we can reasonably make the same assumption, and therefore

$$
\frac{d}{d t}\left[L_{\|}(t)+L_{\perp}(t)\right]=0 .
$$

This last equation means that all building material used by the newer longitudinal array comes from the already existing transverse one.

\section{Steady-state solution}

In order to find a way to control the polarization of the tubulin, and hence the reorientation mechanism, we study the steady-state version of the moment Eqs. (4.47-4.50). If we isolate $r_{c} M_{\|}$and $r_{c} M_{\perp}$ from the four equations we obtain

$$
R_{n}^{\|}=r_{c} M_{\|}=\frac{r_{c}^{2}}{\alpha V^{+}} L_{\|}
$$

and

$$
R_{n}^{\perp}=r_{c} M_{\perp}=\frac{r_{c}^{2}}{V^{+}} L_{\perp} .
$$

From Eq. (4.53) we can observe that multiplying $v^{+}$by $\alpha$ for the longitudinal growing speed is equivalent to divide $r_{c}$ by $\sqrt{\alpha}$.

If we define

$$
\lambda_{\| / \perp} \equiv \frac{L_{\| / \perp}}{L_{t o t}-L_{f}}, \quad \lambda_{*} \equiv \frac{L_{*}}{L_{t o t}-L_{f}},
$$

and we divide Eq. (4.53) by (4.54), by making use of Eqs. (4.9) and (4.10) we obtain the system

$$
\left\{\begin{array}{l}
\frac{\lambda_{\perp}}{\lambda_{\|}}=\frac{\frac{1}{2} \lambda_{*}+\lambda_{\|}}{\alpha\left(\frac{1}{2} \lambda_{*}+\lambda_{\perp}\right)}, \\
\lambda_{\|}+\lambda_{\perp}=1 .
\end{array}\right.
$$


It is important to underline that condition (4.22) imposes $\left(L_{t o t}-L_{f}\right) / L_{t o t} \ll 1$. Indeed, the sudden release of all the tubulin of a microtubule after a catastrophe increases the availability of free tubulin, see also Eq. (4.21). Nevertheless, our choice of $L_{*}$ is still such that $\lambda_{*}<1$, see Table 4.1. With some simple algebra we can solve the system to find

$$
\begin{aligned}
& \lambda_{\|}=\frac{(\alpha-1)-\frac{1}{2} \lambda_{*}(\alpha+1)+\sqrt{\left[(\alpha-1)+\frac{1}{2} \lambda_{*}(\alpha+1)\right]^{2}-2 \lambda_{*}(\alpha-1)}}{2(\alpha-1)}, \\
& \lambda_{\perp}=\frac{(\alpha-1)+\frac{1}{2} \lambda_{*}(\alpha+1)-\sqrt{\left[(\alpha-1)+\frac{1}{2} \lambda_{*}(\alpha+1)\right]^{2}-2 \lambda_{*}(\alpha-1)}}{2(\alpha-1)} .
\end{aligned}
$$

We divide both sides of Eq. (4.57) by $(\alpha-1)+\frac{1}{2} \lambda_{*}(\alpha+1)$ and we obtain

$$
\frac{\lambda_{\perp}}{(\alpha-1)+\frac{1}{2} \lambda_{*}(\alpha+1)}=\frac{1-\sqrt{1-\frac{2 \lambda_{*}(\alpha-1)}{\left[(\alpha-1)+\frac{1}{2} \lambda_{*}(\alpha+1)\right]^{2}}}}{2(\alpha-1)} .
$$

We observe that the second term in the square root is

$$
\frac{2 \lambda_{*}(\alpha-1)}{\left[(\alpha-1)+\frac{1}{2} \lambda_{*}(\alpha+1)\right]^{2}}=\frac{2 \lambda_{*}(\alpha-1)}{(\alpha-1)^{2}+\frac{1}{4} \lambda_{*}^{2}(\alpha+1)^{2}+\lambda_{*}(\alpha+1)(\alpha-1)}<1
$$

because at the denominator $\lambda_{*}(\alpha+1)(\alpha-1)>2 \lambda_{*}(\alpha-1)$ if $\alpha>1$. Therefore we can expand the square root

$$
\frac{\lambda_{\perp}}{(\alpha-1)+\frac{1}{2} \lambda_{*}(\alpha+1)}=\frac{1-\left[1-\frac{1}{2} \frac{2 \lambda_{*}(\alpha-1)}{\left[(\alpha-1)+\frac{1}{2} \lambda_{*}(\alpha+1)\right]^{2}}\right]}{2(\alpha-1)}
$$

and hence

$$
\lambda_{\perp} \simeq \frac{1}{2} \frac{\lambda_{*}}{(\alpha-1)+\frac{1}{2} \lambda_{*}(\alpha+1)}
$$

Consequently

$$
\lambda_{\|} \simeq 1-\frac{1}{2} \frac{\lambda_{*}}{(\alpha-1)+\frac{1}{2} \lambda_{*}(\alpha+1)} .
$$

By plugging Eqs. (4.58) and (4.59) into Eqs. (4.53) and (4.54), we obtain the number of microtubules in the longitudinal and in the transverse directions

$$
M_{\|}=\frac{R_{n}}{r_{c}} \frac{1}{\lambda_{*}+1}\left[\frac{1}{2} \lambda_{*}\left(1-\frac{1}{(\alpha-1)+\frac{1}{2} \lambda_{*}(\alpha+1)}\right)+1\right],
$$




$$
M_{\perp}=\frac{R_{n}}{r_{c}} \frac{\frac{1}{2} \lambda_{*}}{\lambda_{*}+1}\left(1+\frac{1}{(\alpha-1)+\frac{1}{2} \lambda_{*}(\alpha+1)}\right) .
$$

With this result we can control the amount of tubulin and the number of microtubules in the transverse array by acting on the probability of dispersed nucleation, and hence we can control the efficiency of the reorientation, see Figure 4.7 .

More generally, we have proven that when two populations of individuals (microtubules) compete for the same pool of building material (tubulin) with a birth mechanism proportional to the overall age (length) of a population, a small bias in the ageing rate (growing speed) causes a significant unbalance in the number of individuals in the two populations, as well as in their overall age (length).

It is interesting to study the two limit cases of $\lambda_{*} \rightarrow 0$ - i.e. nucleation is all microtubule-based, and $\lambda_{*} \rightarrow \infty$ - i.e. nucleation is all dispersed. In the first case we observe that

$$
\begin{gathered}
\lim _{\lambda_{*} \rightarrow 0} \lambda_{\|}=1, \\
\lim _{\lambda_{*} \rightarrow 0} \lambda_{\perp}=0, \\
\lim _{\lambda_{*} \rightarrow 0} M_{\|}=\frac{R_{n}}{r_{c}}, \\
\lim _{\lambda_{*} \rightarrow 0} M_{\perp}=0,
\end{gathered}
$$

i.e. all tubulin is polarized in the longitudinal direction, and then we observe full reorientation of the array from the transverse to the longitudinal direction. In the opposite limit

$$
\begin{aligned}
\lim _{\lambda_{*} \rightarrow \infty} \lambda_{\|} & =\frac{\alpha}{\alpha+1}, \\
\lim _{\lambda_{*} \rightarrow \infty} \lambda_{\perp} & =\frac{1}{\alpha+1}, \\
\lim _{\lambda_{*} \rightarrow \infty} M_{\|} & =\frac{1}{2} \frac{R_{n}}{r_{c}}, \\
\lim _{\lambda_{*} \rightarrow \infty} M_{\perp} & =\frac{1}{2} \frac{R_{n}}{r_{c}},
\end{aligned}
$$

meaning that although in such a limit the isotropy of the dispersed nucleation imposes that the final number of microtubules of the two populations is the same, the $\alpha>1$ bias in the growing speed still produces a slight tubulin polarization in the longitudinal direction, with the consequence of producing slightly longer longitudinal microtubules than transverse, see Figure 4.7. 

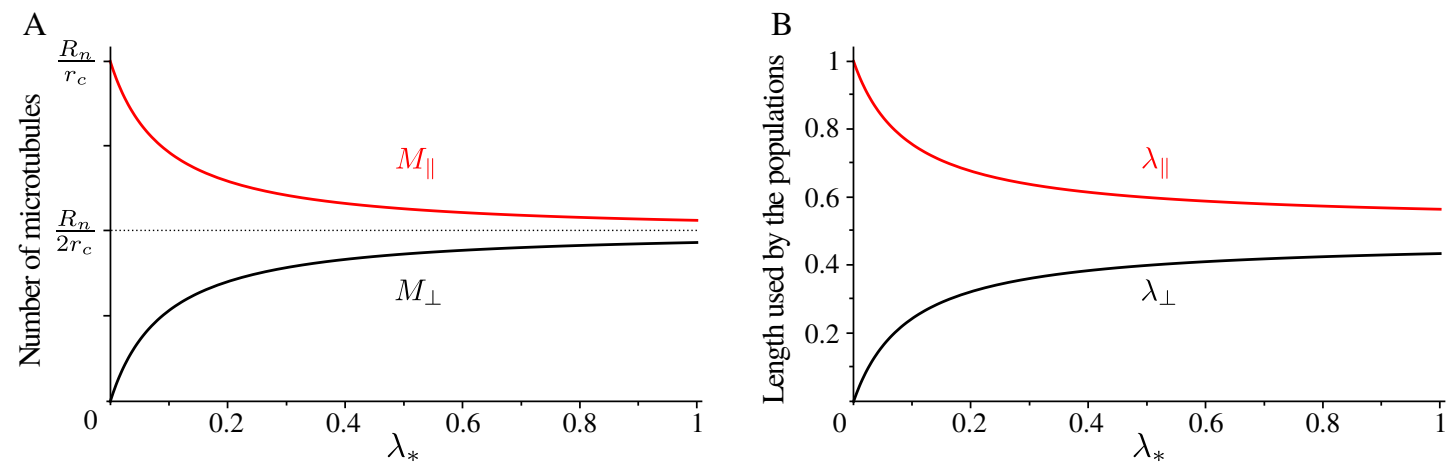

Figure 4.7: (A) Number of microtubules and (B) non-dimensional length used by microtubules populations as functions of the propensity length for dispersed nucleation, with $\alpha=1.1$.

Furthermore, if we plug Eq. (4.58) into the steady-state version of Eq. (4.50), we obtain an expression for the amount of free tubulin, as

$L_{f}^{2}-\left[L_{t o t}-L_{v}+L_{*}-\frac{v^{+} R_{n}}{r_{c}^{2}}\left(\alpha+\frac{1}{2} \frac{L_{*}}{L_{t o t}-L_{f}}(\alpha+1)\right)\right] L_{f}-L_{v}\left(L_{t o t}+L_{*}\right)=0$,

where, once more, we did not express $R_{n}$ in terms of $L_{f}$, and where in the second coefficient we observe a direct dependency on $L_{f}$. However, this expression bears similarities with Eq. (4.21) for the free tubulin of the initial transverse array, i.e.

$$
L_{f}^{2}-\left(L_{t o t}-L_{v}-\frac{v^{+} R_{n}}{r_{c}^{2}}\right) L_{f}-L_{v} L_{t o t}=0
$$

The term $\alpha+\frac{1}{2} \frac{L_{*}}{L_{t o t}-L_{f}}(\alpha+1)$ if Eq. (4.62) is smaller than two: indeed, we already observed for the initial array that in our $L_{*} \ll L_{t o t}$ limit, the relation $L_{*}<L_{t o t}-L_{f}$ still holds. Here, given the increased average growing speed of all microtubules, we can reasonably assume that if there is a change in $L_{f}$ with respect to the initial configuration, this change can only make $L_{f}$ become smaller, and consequently $L_{*}<L_{t o t}-L_{f}$ for the final configuration as well. Hence, the second coefficient of both equations is dominated by the factor $L_{t o t}-L_{v}$, see Table 4.1. Similarly, the third coefficient of both equations is similar, as $L_{t o t} \gg L_{*}$. This means that the overall change in the amount of free tubulin from the initial transverse array to the final steady-state with both microtubule populations is neglectable. This proves that our choice of considering $L_{f}=$ const is reasonable. As a consequence, $L_{\|}+L_{\perp}$ is constant in time, as the relation $L_{\|}+L_{\perp}=L_{t o t}-L_{f}$ holds. 


\section{Time-dependent solution}

In this section we look for an expression for the time scale of the reorientation of the array from the transverse to the longitudinal direction to the growth direction of the cell. We focus on Eqs. (4.47) and (4.49), with their respective boundary conditions

$$
M_{\|}(0)=0=L_{\|}(0) .
$$

By plugging these boundary conditions in Eqs. (4.47) and (4.49) we obtain the boundary conditions for the first derivative of both $M_{\|}$and $L_{\|}$

$$
\begin{gathered}
\left.\frac{d M_{\|}(t)}{d t}\right|_{t=0}=R_{n} \frac{\frac{1}{2} L_{*}}{L_{t o t}-L_{f}+L_{*}}, \\
\left.\frac{d L_{\|}(t)}{d t}\right|_{t=0}=0 .
\end{gathered}
$$

Eqs. (4.47) and (4.49) can be decoupled to obtain

$$
\frac{d^{2} L_{\|}(t)}{d t^{2}}+2 r_{c} \frac{d L_{\|}(t)}{d t}+\left(r_{c}^{2}-\alpha V^{+} \widetilde{R}_{n}\right) L_{\|}(t)=\alpha V^{+} \widetilde{R}_{n} \frac{1}{2} L_{*},
$$

and

$$
\frac{d^{2} M_{\|}(t)}{d t^{2}}+2 r_{c} \frac{d M_{\|}(t)}{d t}+\left(r_{c}^{2}-\alpha V^{+} \widetilde{R}_{n}\right) M_{\|}(t)=r_{c} \widetilde{R}_{n} \frac{1}{2} L_{*},
$$

where we defined

$$
\widetilde{R}_{n}=\frac{R_{n}}{L_{t o t}-L_{f}+L_{*}}
$$

Eqs. (4.63) are second order non-homogeneous differential equations, the solutions of which are

$$
\begin{aligned}
L_{\|}(t)=\frac{1}{2} L_{*} \frac{\alpha V^{+} \widetilde{R}_{n}}{r_{c}^{2}-\alpha V^{+} \widetilde{R}_{n}}+\frac{1}{2} L_{*} \frac{\sqrt{\alpha V^{+} \widetilde{R}_{n}}}{2\left(r_{c}^{2}-\alpha V^{+} \widetilde{R}_{n}\right)} \\
\times\left[\left(r_{c}-\sqrt{\alpha V^{+} \widetilde{R}_{n}}\right) e^{-\left(r_{c}+\sqrt{\alpha V^{+} \widetilde{R}_{n}}\right) t}\right. \\
\left.-\left(r_{c}+\sqrt{\alpha V^{+} \widetilde{R}_{n}}\right) e^{-\left(r_{c}-\sqrt{\alpha V^{+} \widetilde{R}_{n}}\right) t}\right],
\end{aligned}
$$




$$
\begin{aligned}
& M_{\|}(t)=\frac{1}{2} L_{*} \frac{r_{c} \widetilde{R}_{n}}{r_{c}^{2}-\alpha V^{+} \widetilde{R}_{n}}+\frac{1}{2} L_{*} \frac{\widetilde{R}_{n}}{2\left(r_{c}^{2}-\alpha V^{+} \widetilde{R}_{n}\right)} \\
& \times\left[\left(\sqrt{\alpha V^{+} \widetilde{R}_{n}}+r_{c}\right) e^{-\left(r_{c}-\sqrt{\alpha V^{+} \widetilde{R}_{n}}\right) t}\right. \\
& \left.-\left(\sqrt{\alpha V^{+} \widetilde{R}_{n}}-r_{c}\right) e^{-\left(r_{c}+\sqrt{\alpha V^{+} \widetilde{R}_{n}}\right) t}\right] .
\end{aligned}
$$

These expressions define the time scale of the reorientation process, i.e.

$$
t_{\text {reor }} \propto\left(r_{c}-\sqrt{\alpha V^{+} \widetilde{R}_{n}}\right)^{-1}
$$

To highlight all dependencies of $t_{\text {reor }}$ on the model parameters, we can conveniently rewrite $\alpha V^{+} \widetilde{R}_{n}$ by expressing all quantities as functions of $L_{f}$, and by making use of Eq. (4.62) we find

$$
\alpha V^{+} \widetilde{R}_{n}=\alpha v^{+} r_{n} \frac{L_{f}}{L_{f}+L_{v}} \frac{L_{f}^{a}}{L_{f}^{a}+L_{v}^{a}} \frac{1}{L_{t o t}-L_{f}+L_{*}}=r_{c}^{2} \frac{\alpha}{\alpha+\frac{1}{2} \frac{L_{*}}{L_{t o t}-L_{f}}(\alpha+1)}
$$

Thus, the time scale of the reorientation can be written now as

$$
t_{\text {reor }} \propto\left[r_{c}\left(1-\sqrt{\frac{\alpha\left(L_{t o t}-L_{f}\right)}{\alpha\left(L_{t o t}-L_{f}\right)+\frac{1}{2} L_{*}(\alpha+1)}}\right)\right]^{-1} .
$$

As we previously discussed, $L_{f}$ is weakly dependent on $r_{c}$. Because of this, Eq. (4.68) reveals that the time scale of the reorientation is inversely proportional to the catastrophe rate. The interpretation of this counter-intuitive result, is that every time a microtubule undergoes a catastrophe, it releases to the free tubulin pool an amount of tubulin equal to its length. Therefore, the amount of building material available for the new array increases with higher rate, and so does the speed of reorientation.

\section{Polarization and transverse suppression}

Contrarily to Section 4.1, here we can analytically calculate the reorientation polarization and transverse suppression. Indeed, from Eqs. (4.58-4.61) we obtain

$$
\mathcal{P}_{M}=\frac{M_{\|}-M_{\perp}}{M_{\|}+M_{\perp}}=\frac{(\alpha-1)\left(1-\frac{1}{2} \lambda_{*}\right)}{\left(1+\lambda_{*}\right)\left[(\alpha-1)+\frac{1}{2} \lambda_{*}(\alpha+1)\right]},
$$


and

$$
\mathcal{P}_{L}=\frac{L_{\|}-L_{\perp}}{L_{\|}+L_{\perp}}=\frac{(\alpha-1)\left(1+\frac{1}{2} \lambda_{*}\right)}{(\alpha-1)+\frac{1}{2} \lambda_{*}(\alpha+1)} .
$$

As expected, both reorientation parameters are close to 1 when $\lambda_{*}$ is small, see Figure 4.8, whilst they rapidly decay to 0 for higher values of $\lambda_{*}$. These expressions for the polarization also set the value for $\alpha$ in order to have reorientation for fixed $\lambda_{*}$. Indeed, let e.g. $\bar{P}$ be a certain value for the lenght polarization. Then, in order to have $\mathcal{P}_{L}>\bar{P}, \alpha$ needs to be such that

$$
\alpha>\frac{\lambda_{*}+\left(1-\frac{1}{2} \lambda_{*}\right)(1-\bar{P})}{\left(1+\frac{1}{2} \lambda_{*}\right)(1-\bar{P})} .
$$

Figure 4.9 shows that for small $\lambda_{*}$, a little difference between the growing speeds of the two populations is enough to yield to longitudinal polarization.

Similarly, we can calculate the transverse suppression:

$$
\mathcal{R}_{M}=\frac{M_{\perp}^{0}-M_{\perp}}{M_{\perp}^{0}+M_{\perp}}=\frac{\left(\frac{1}{2} \lambda_{*}\right)^{2}(\alpha+1)+\frac{1}{2} \lambda_{*}(2 \alpha-1)+(\alpha-1)}{3\left(\frac{1}{2} \lambda_{*}\right)^{2}(\alpha+1)+\frac{1}{2} \lambda_{*}(4 \alpha-1)+(\alpha-1)}
$$

and

$$
\mathcal{R}_{L}=\frac{L_{\perp}^{0}-L_{\perp}}{L_{\perp}^{0}+L_{\perp}}=\frac{\alpha\left(1+\frac{1}{2} \lambda_{*}\right)-1}{\alpha\left(1+\frac{1}{2} \lambda_{*}\right)-1+\lambda_{*}} .
$$

Figure 4.10 shows that in the case of the transverse suppression the effect of the bias in the speed is to almost completely remove the initial transverse array.
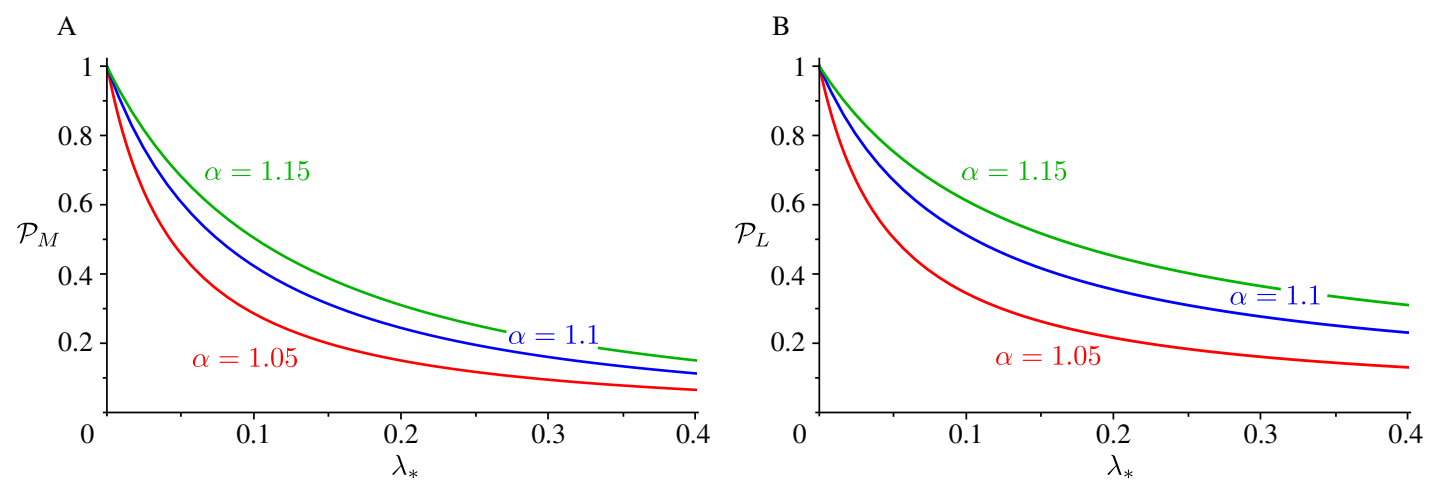

Figure 4.8: Polarization for (A) the microtubules, and (B) the tubulin as a function of the propensity length for dispersed nucleation for $\alpha=1.05$ (red), $\alpha=1.1$ (blue), and $\alpha=1.15$ (green). 


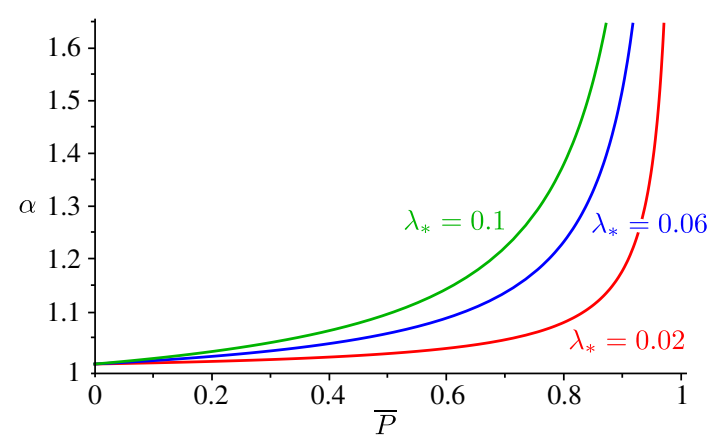

Figure 4.9: Minimum speed bias $\alpha$ required to have length polarization greater than $\bar{P}$, for $\lambda_{*}=0.02$ (red), $\lambda_{*}=0.06$ (blue), and $\lambda_{*}=0.1$ (green).
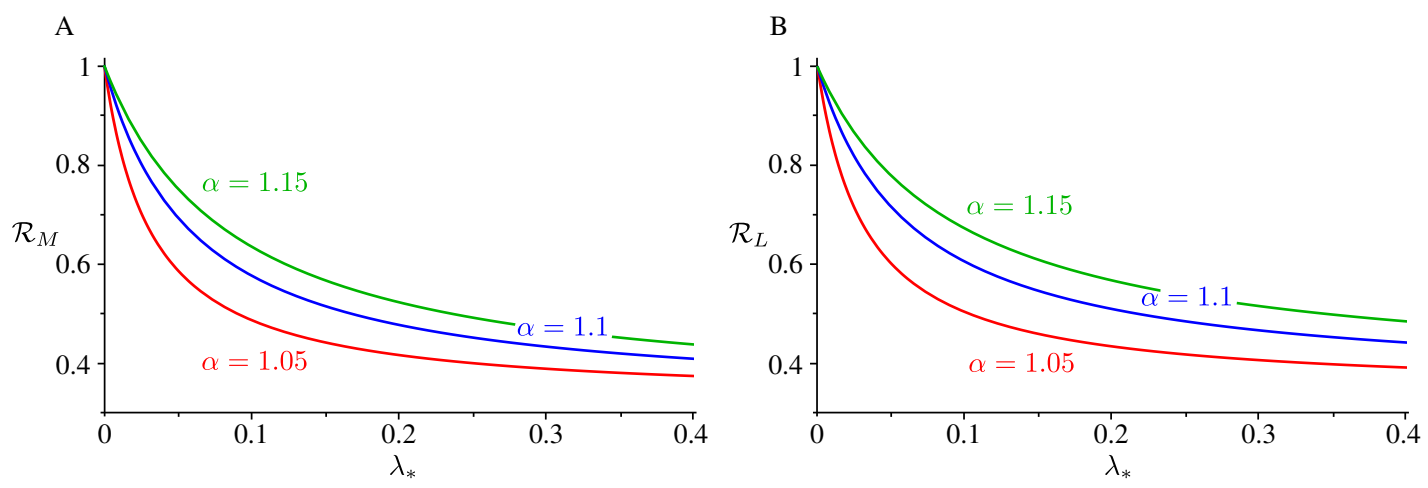

Figure 4.10: Suppression for (A) microtubules number and (B) length as a function of the propensity length for dispersed nucleation for $\alpha=1.05$ (red), $\alpha=1.1$ (blue), and $\alpha=1.15$ (green). 


\subsection{Computational approach}

This section is divided in two subsections. In the first one, we computationally test the theoretical predictions of Section 4.2 in the case in which shrinking microtubules do not completely depolymerize suddenly after the catastrophe. In other words, we test if our findings can be generalized to the same model with also shrinking microtubules. In the second section, instead, we reintroduce the severing mechanism described in Section 4.1. There, we computationally study if the combination of severing and biased speed affects the efficiency and the time of the reorientation.

\section{Without severing}

In order to test whether our theoretical predictions can be generalized to the full model described in Section 4.2 with shrinking microtubules as well, we perform stochastic simulations of the model.
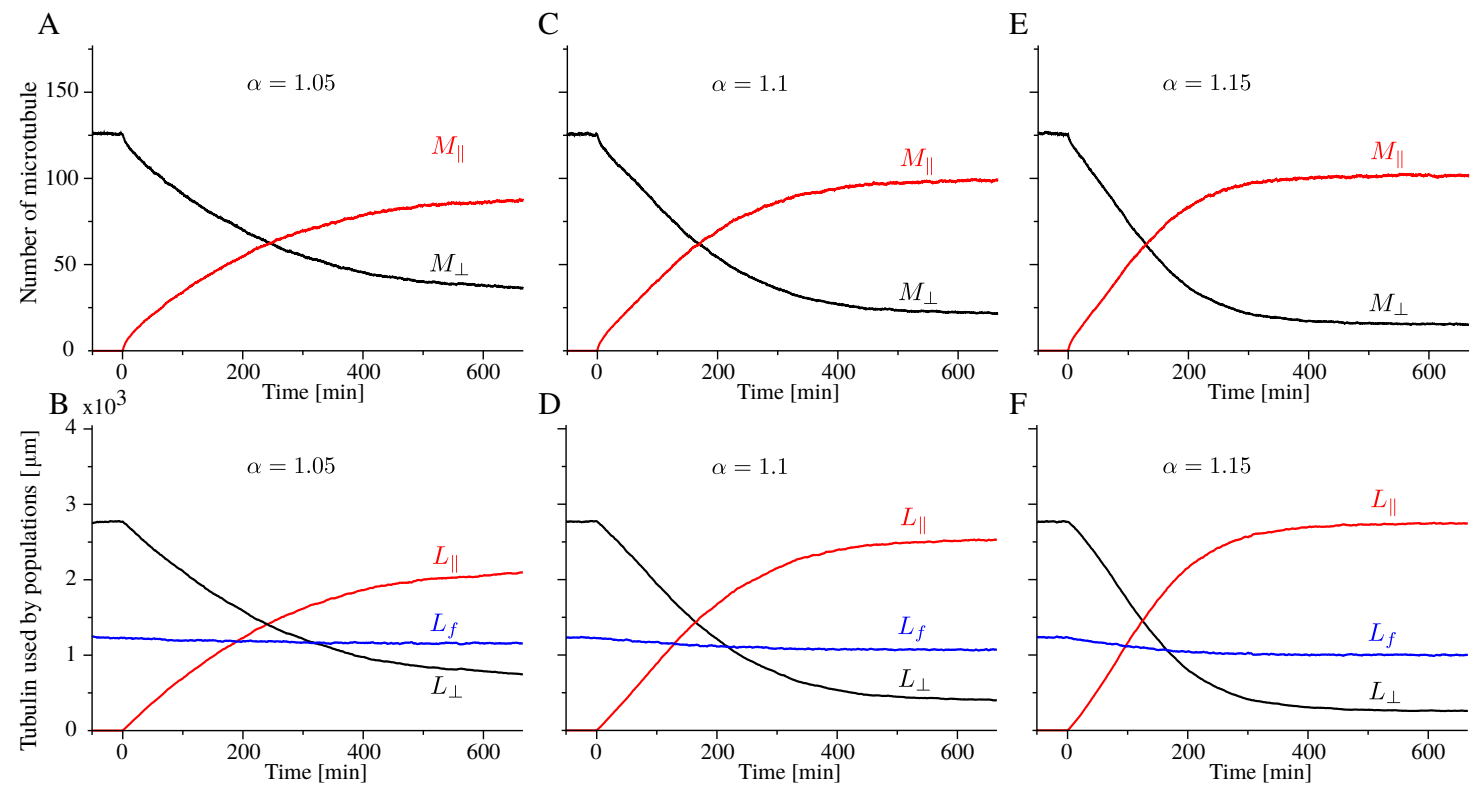

Figure 4.11: Time evolution of (A, C, E) longitudinal (red) and transverse (black) microtubules, and (B, D, F) tubulin used by the longitudinal population (red), the transverse population (black), and the free tubulin (blue), for $\alpha=1.05$ (A, B), $\alpha=1.1(\mathrm{C}, \mathrm{D})$, and $\alpha=1.15(\mathrm{E}, \mathrm{F})$.

We run $N=10^{3}$ stochastic simulations consisting first in creating the initial transverse array through the mechanism described in Section 4.1. At time $t=0$ we 
switch to the full model, with nucleation occurring in the longitudinal direction as well as in the transverse, with model parameters given by Table 4.1, except for the severing rate, as we are discussing the model without severing, and hence $r_{s}=0$.

Figure 4.11 shows that, for different $\alpha$ values, when the steady-state is reached both the number of microtubule and the overall length are polarized more in the longitudinal direction rather than in the transverse. The figure also reveals that increasing $\alpha$ has a double effect: indeed, not only it increases the efficiency, but it also shortens the time scale of the reorientation, in agreement with our theoretical predictions. Furthermore, small values of $\alpha$ do not seem to have an influence on the change of the free tubulin of the system (Figure 4.11B), while when as $\alpha$ increases, $L_{f}$ slightly decreases (Figure $4.11 \mathrm{~F}$ ). Therefore, for higher values of $\alpha$, we can no longer consider the pool of free tubulin as a constant length for the estimate of the reorientation time in Eq. (4.68).

Figure 4.12, instead, shows the time dependency of $L_{\|}(t)$ for different catastrophe rates. As expected from Eq. (4.68), we observe a very fast decrease of the time needed to reach the steady-state as $r_{c}$ increases, with the $r_{c}=0.005$ events $/ \mathrm{s}$ case where $L_{\|}$needs many hours to reach the plateau. However, the increase of the catastrophe rate is also correlated to a lower steady-state value for the longitudinal length $L_{\|}$. Intuitively, we can argue that this is due to the fact that the mean length of a microtubule is inversely proportional to the catastrophe rate, and so is the sum of the lengths of all microtubules. However, we still lack a full quantitative description of the effect of the dynamic parameters - and specifically of the catastrophe rate, on the efficiency of the reorientation and on the reorientation time. These preliminary data suggest the existence of an optimal value for $r_{c}$ in order to have efficient reorientation in a short time, and future investigations will be performed toward that direction.

\section{With severing}

In the previous sections we have observed that a good probability of rescue after severing together with preferential severing is required in order to quickly reach the steady-state. Unfortunately, these two ingredients are not enough to have a sustained reorientation. We have also observed how a little difference in the growing speed of the two populations, together with the microtubule-based nucleation mechanism can maintain the new orientation. In this case, however the theoretically predicted reorientation time is in general slow compared to the experimentally observed one. Here, we study both the efficiency and the time of the reorientation after including in the model both severing and biased speed.

We have already observed that an analytical solution of the model presented in Section 4.1 is not reachable. Therefore, we set up stochastic simulations of the 


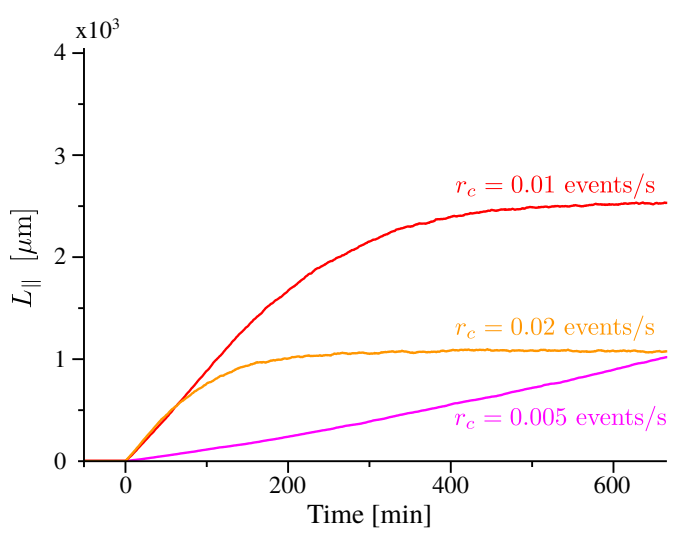

Figure 4.12: Time evolution of $L_{\|}$for $r_{c}=0.005$ events/s (magenta), $r_{c}=0.01$ events/s (red), and $r_{c}=0.02$ events/s (orange).

new model, that includes both preferential severing and asymmetry in the growing speed of microtubules belonging to the two different populations.

Our goal, here, is to test whether or not the model introduced in Section 4.1, with the addition of the small difference in the growing speeds of the two populations, leads to a complete and fast reorientation. As we want to keep the difference between the two speeds small, we set $\alpha=1.1$, while we make use of dynamic parameters listed in Table 4.1. Then, as we already did in the first section of the chapter, we perform a sensitivity analysis in which we separately tune $q$ and $p^{+}$from 0 to 1 in order to find the optimal combination of these two probabilities for the reorientation of the array. Figures 4.13 and 4.14 shows the polarization and the transverse suppression for both microtubule number and length. Although this case, like in Section 4.1, reveals that a more efficient and fast reorientation requires high values of both $q$ and $p^{+}$, here we can observe that the lighter areas in all heat maps are wider than in the previous case. In particular, we can observe a good degree of reorientation for $p^{+}$comprises between 0 and 0.25 , i.e. in the biological range, for almost every value of $q$. In particular, it is interesting to notice that in such an area, polarization and suppression seem to be not strongly dependent on $q$. This suggests that, even though the preferential severing plays an important role in the amplification phase of the reorientation to boost the creation of longitudinal microtubules, in presence of biased speed it is not stricty necessary to maintain the new longitudinal array. Figure 4.14 also shows that here the area in the $\left(q, p^{+}\right)$ space such that the reorientation occur is bigger than in the case of the model of Section 4.1, see Figure 4.4.

Figure 4.15 shows the time behaviour of the number and the total length of microtubules belonging to the different populations. The combination of preferential 

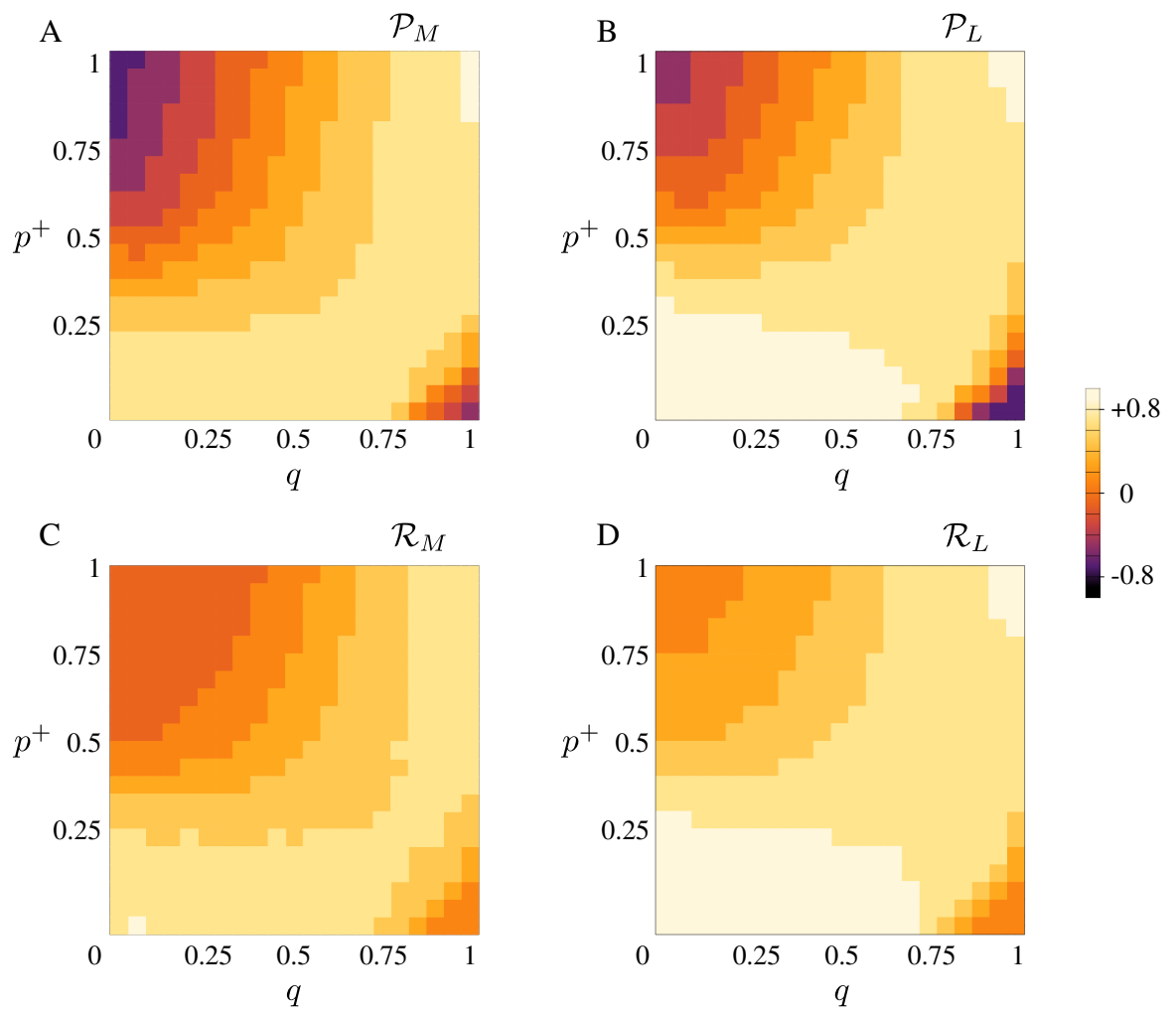

Figure 4.13: (A) Microtubule number polarization, (B) microtubule length polarization, (C) transverse number suppression, (D) and transverse length suppression as functions of $q$ and $p^{+}$. Lighter colors correspond to a more efficient reorientation. Results are averaged over $N=10^{3}$ simulations.

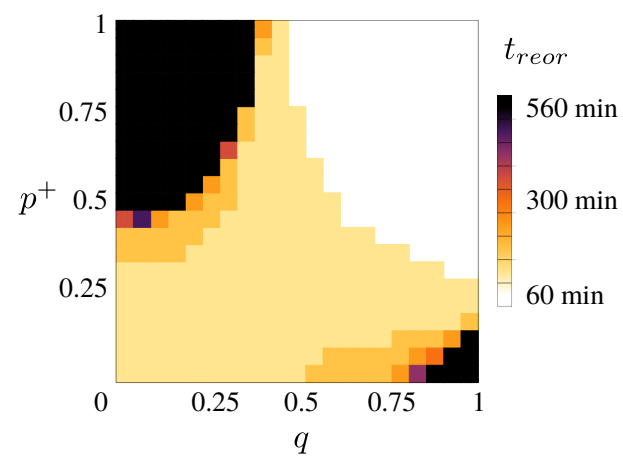

Figure 4.14: Transverse-to-longitudinal reorientation time as a function of $q$ and $p^{+}$. Lighter colors correspond to a faster reorientation. Results are averaged over $N=10^{3}$ simulations. 

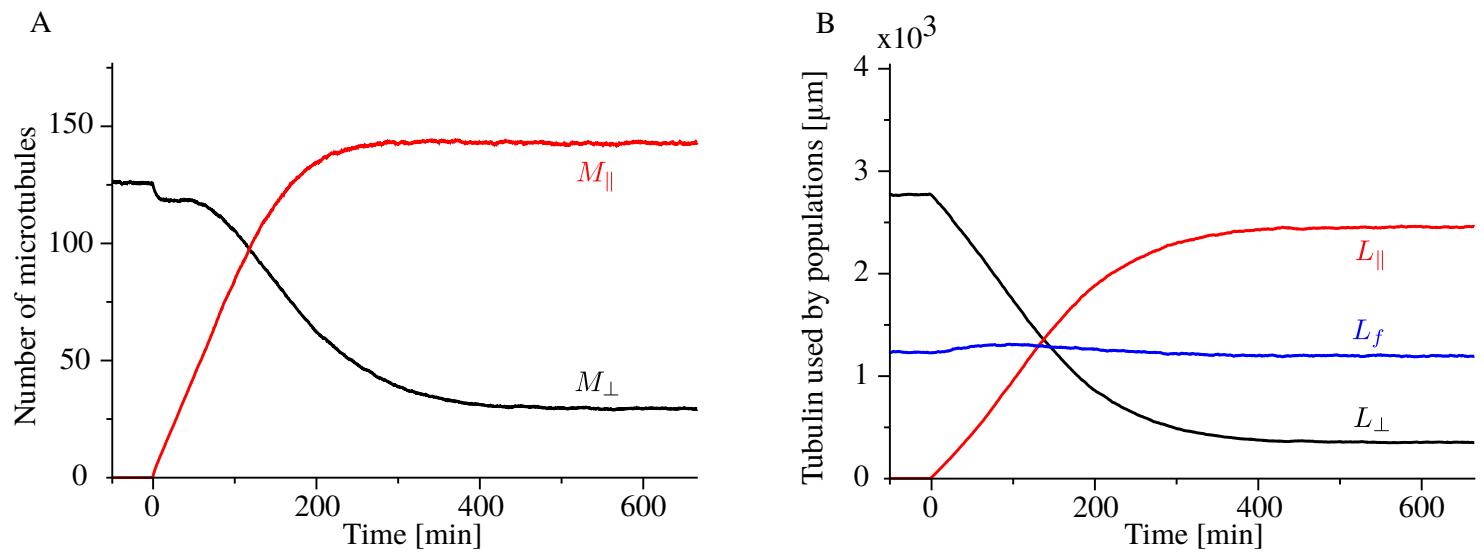

Figure 4.15: Time evolution of (A) longitudinal (red) and transverse (black) microtubules, and (B) tubulin used by the longitudinal population (red), the transverse population (black), and the free tubulin (blue), averaged over $N=10^{3}$ simulations.

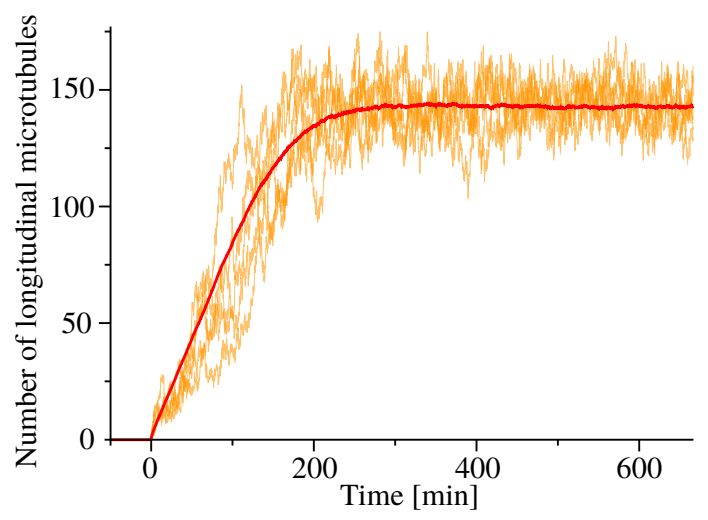

Figure 4.16: Time evolution of $M_{\|}$averaged over $N=10^{3}$ simulations (red line), and in five different single simulations (orange lines). Besides the statistical noise, every single simulation reaches the same steady-state value. 
severing and biased speed has then a double effect: it makes the reorientation more efficient by increasing the number of longitudinal microtubules at the steady-state and suppressing the transverse, and it makes the reorientation occur faster, see also Figure 4.16. This result suggests that our model is a possible candidate to explain the underlying mechanism behind the reorientation of the cortical microtubule array and its maintenance in the newer direction observed in the in vivo performed experiments. Moreover, Figure 4.15B also shows that even in this case we cannot observe any appreciable difference between the amount of free tubulin at the beginning and at the end of the process, proving again that the building material used by the longitudinal array comes from the original transverse one.

\subsection{Conclusions}

In this chapter, we have studied the long-time behaviour of the cortical microtubule array during the transverse-to-longitudinal reorientation process. Our aim was to test our initial hypothesis: is the asymmetry induced by the preferential severing for the longitudinal microtubules enough to explain the full reorientation and the maintenance of the new longitudinal array? Our computer simulations of Section 4.1 have revealed that, although the preferential severing indeed creates polarization of number and length of microtubules in the longitudinal direction, such a polarization is not large enough, as the system still exhibits a substantial amount of microtubules directed transversally to the growth direction of the cell. Indeed, we do not observe a considerable transverse suppression. A possible explanation of this outcome is the increased possibility of creation of new transverse microtubules due to the severing event with respect to that of the system in the initial state, in which only transverse microtubules where present. Furthermore, even though the experiments have revealed a strong correlation between new microtubules and longitudinal microtubules (Lindeboom et al., Science, 2013), and hence preferential severing for the longitudinal in the first stage of the reorientation process, for longer times we expect this correlation to drop, as the microtubules belonging to the initial transverse array eventually disappear due to their finite lifetime. Therefore, our conclusion is that the preferential severing cannot explain the maintenance of the new longitudinal array for two separate reasons. Firstly, it still increases the creation of new transverse microtubules. Secondly, for long time periods, the correlation between new and longitudinal microtubules vanishes, as the initial microtubules all disappear due to their finite lifetime, and new microtubules are nucleated in both directions. In this case, we expect not to find any preferential severing for longitudinal microtubules for long time periods. Therefore, an interesting research question to address could be to experimentally quantify the time evolution of the 
correlation between new and longitudinal microtubules, in order to understand whether or not the preferential severing still takes place after long periods of time. However, it is important to underline that the preferential severing is still an important factor in the first stage of the reorientation process, as it allows the number of longitudinal microtubules to quickly amplify, see Chapters 2 and 3.

To understand which asymmetry can explain the reorientation of the cortical array and the maintenance of the new direction we made a new hypothesis: transverse and longitudinal microtubules have slightly different dynamic parameters. To test this hypothesis, in Section 4.2 we studied the model introduced in Section 4.1 with two changes: no severing events, and growing speed for the longitudinal microtubules slightly larger than that for the transverse. We analytically showed that this small bias in the growing speed coupled with a strong correlation between tubulin polarization and direction of the newly-nucleated microtubules, explains both the reorientation of the array and the maintenance of the new longitudinal one. Yet, with only this ingredient, the reorientation process occurs in a considerably larger time scale with respect to the experimentally measured one. Intriguingly, we observed that the same result could be obtained by introducing a bias in the catastrophe rate, i.e. by slightly reducing the catastrophe rate for longitudinal microtubules. This suggests that a small difference that breaks the symmetry between transverse and longitudinal microtubules in any of their dynamic parameters, can lead to macroscopic effects concerning the number and the length polarization and suppression. Unfortunately, at the moment there are no experimental observations of such an asymmetry that could confirm our theoretical predictions. Indeed, with our model we do not claim that the reason of the reorientation is the presence of a difference in the dynamic parameters, but we do claim that if the difference exists, it is an excellent candidate to be the responsible of the reorientation.

In Section 4.3, instead, we put together the bias in the speed and the severing for microtubules. Our aim was to test whether the results obtained in Section 4.2 were still valid in the case of the addition of the severing mechanism, and to check whether it was possible to have a fast reorientation with the two ingredients together. We found out that, with the severing mechanism, not only the reorientation was still possible, but it was faster than in the case of the model studied in the first two sections of this chapter. Moreover we observed that, as expected, the preferential severing for the longitudinal microtubules is not a necessary ingredient to explain the final number and length polarization in the longitudinal direction. Indeed, we observed a weak dependency of polarization and transverse suppression on $q$, for $p^{+}$in the biological range of values. These results also showed that microtubule severing and small difference in the speed contribute to the reorientation of the cortical array and in the maintenance of the new one in two distinct ways: the former speeds up the reorientation process, whilst the latter makes it occur and, 
furthermore, it maintains the new longitudinal array. 


\section{Microtubule-based actin transport in a three- dimensional confinement}

In the previous chapter we investigated microtubules as independent components of the cytoskeleton. In other words, we modelled the plant cytoskeleton as if it was only composed by microtubules, ignoring the other two polymers present there, i.e. actin and intermediate filaments, and ignoring the interaction between different cytoskeletal components. Here, instead, we introduce a system composed of dynamic microtubules interacting with diffusing actin.

The interaction between actin and microtubules is very important for many cellular mechanisms, such as maintenance of cell shape, cell division and migration, cell polarization, cellular wound healing, and many others (Siegrist \& Doe, Genes Dev., 2007 - Huber et al., Curr. Opin. Cell. Biol., 2015 - Dogterom \& Koenderink, Nat. Rev. Mol. Cell Biol., 2018). Therefore, understanding the underlying mechanisms and the consequences of such an interaction plays an important role in the investigation of cellular and cytoskeletal processes. However, although actin and microtubules have been very well studied independently, very little has been explored about how these two polymers interact with each other.

The experiments reviewed in this chapter have been designed and carried out by Celine Alkemade, Marileen Dogterom and Gijsje H. Koenderink from Technische Universiteit Delft (The Netherlands). 
In this chapter, we theoretically study the consequences of the interaction between actin filaments and microtubules in three dimensional confinement. In Section 5.1, we present the in vitro experiments that observed the interaction between actin and microtubules mediated by a class of linking proteins called cytolinkers. The experiments highlight two important features caused by the interaction: a change in the dynamic properties of microtubules and microtubulebased transport of actin. In Section 5.2 we introduce a stochastic model of microtubules undergoing dynamic instability in a three dimensional cell with freely diffusing actin in it. As the equations of the model exhibit a high degree of complexity we can only numerically solve them to highlight their main features. In Section 5.3 we analytically solve the system in the limit of fast diffusion for the actin filaments. This allows us to understand what the consequences of the changed dynamics of microtubules are for the steady-state length distribution of the latter. Finally, in Section 5.4 we analytically study the system under the assumption that the actin-microtubule interaction does not change the dynamic properties of the microtubules. In this way, although we still cannot analytically solve the model, we can identify how the system responds to the change of the parameters of the model.

\subsection{Overview of experiments}

\section{Actin filaments}

Actin filaments (F-actin) are one of the components of the eukaryotic cellular cytoskeleton. They are composed by globular actin proteins (G-actin) that organize in a double stranded helix to form the filament, the diameter of which is of about 7 nanometers. The persistence length of an actin filament is of the order of 10 $\mu \mathrm{m}$, whilst its typical length can extend for a few micrometers. Therefore, actin filaments are both smaller and more flexible than microtubules.

In the cytoskeleton, actin filaments form a dynamic network that plays an important role in many cellular processes, such as cell motility, phagocytosis, or receiving extracellular signals to mediate the cell response (Papakonstanti \& Stournaras, Methods Enzymol., 2007). The organization of the actin network is mediated by the so-called crosslinking proteins, that bind actin filaments together and make them interact. Some of these proteins, like for example MACF1, can also bind actin to microtubules (Chen et al., Genes Dev., 2006), suggesting that the organization of the former can be influenced by the dynamics of the latter. 


\section{Experimental observations}

Actin and microtubules can interact in different possible ways. For example, they can interact through steric repulsion, passive crosslinking, microtubule growth guidance by actin bundles, among others (Preciado Lopez et al., Nat. Comm., 2014 - Preciado Lopez, 2015). Here, we focus on the interaction mediated by cytolinkers. Typically, cytolinkers have an actin binding domain to bind to actin, and two distinct ways to bind to microtubules: either they bind to the tip via a link to an EB protein, or to the lattice.

Recent quasi 2D in vitro experiments (Alkemade et al., in preparation) have shown that different engineered cytolinkers can connect actin to microtubules, with different effects. As an example, TipActLong is a cytolinker that binds actin to the lattice of microtubules, see Figure 5.1A, with an interesting effect on the dynamics of the latter. Indeed, they slow down microtubule growth by decreasing their speed, but they simultaneously dramatically suppress the catastrophe rate, see Figure 5.2 , resulting in an overall stabilization of the microtubules. As a consequence, microtubules are helped in growing in dense actin networks. Intriguingly, Figure 5.2 also reveals that in the case of TipActLong, an appreciable change in the dynamics of microtubules occur only when also actin filaments are present.

A

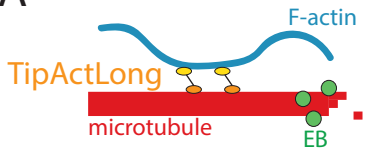

B

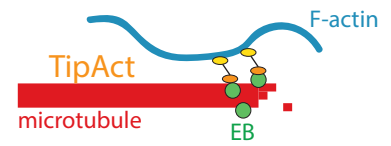

Figure 5.1: Microtubules (red blocks) and actin (blue lines) interacting through cytolinkers. (A) TipActLong binds to microtubule lattice whilst (B) TipAct binds to the microtubule plus end via EB. Courtesy of Celine Alkemade.

On the contrary, experiments performed with the cytolinker TipAct, that links the plus end of microtubules to actin filaments, see Figure 5.1B, have revealed a different effect coming from the interaction. Indeed, it has been observed that on the one hand it still changes the dynamic properties of microtubules, but on the other hand it captures diffusing actin filaments on microtubule plus ends. A captured actin filament is then transported as long as the microtubule grows, or until the actin filament simply detaches from the microtubule.

As regards the change in microtubule dynamics, the experiments have revealed that in presence of TipAct the growing speed of microtubules undergoes a significant decrease with respect to the control case, while it increases the catastrophe rate, effecting in the shortening of the average length of microtubules, see Figure 5.2. 

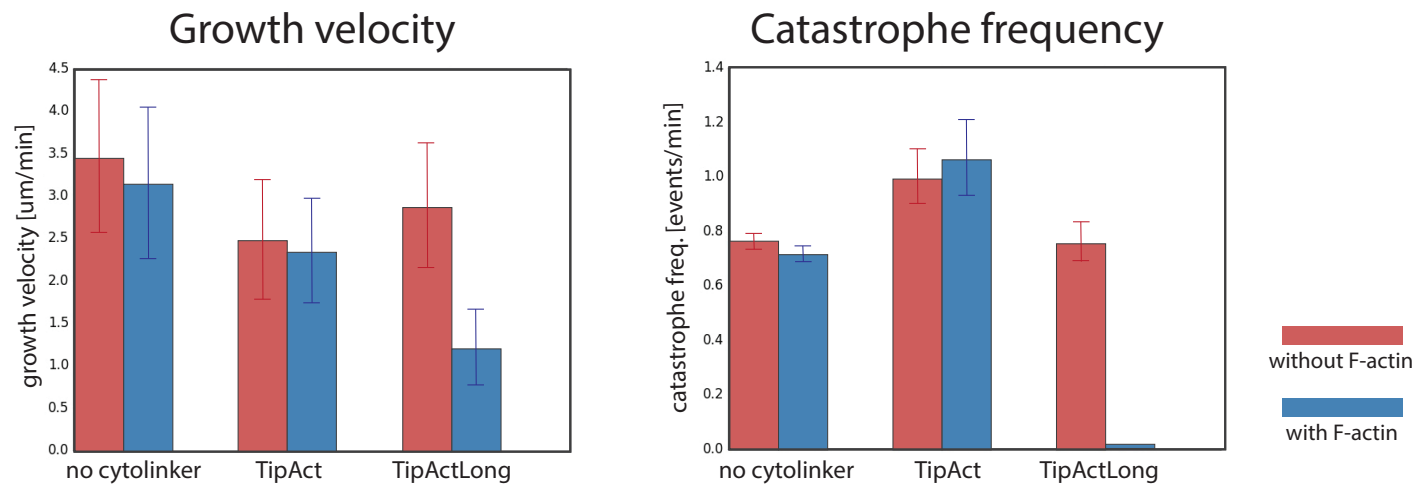

Figure 5.2: Growth speed and catastrophe rate for microtubules in absence of cytolinkers, with TipAct and TipActLong. All measurements are repeated two times: without actin (red histograms), and with actin (blue histograms). Image taken from (Alkemade et al., in preparation).

Intriguingly, it seems that there is no substantial difference in both growing speed and catastrophe rate when actin is present with respect to when it is absent, still in presence of TipAct, suggesting that the change in the dynamics of microtubules is due to changes in the biochemistry of the system rather than to steric interactions between microtubules and actin.

Concerning the actin transport, the kymograph in Figure 5.3 shows that the localization of actin is maintained at microtubule plus end, confirming the occurrence of actin transport by the microtubule tip. The capture of actin from microtubules occurs when, due the dynamics of the microtubule and the observed diffusion of actin in the sample, the latter is close enough to the plus end of the former to allow the binding through TipAct. Typically, a microtubule binds to actin before reaching a length of about $7 \mu \mathrm{m}$. The experiments show that the actin transport occur on average for 70 seconds before the unbinding from the transporting microtubule. The unbinding can occur both because of the detachment of the actin or induced by a microtubule catastrophe. In a very few cases, however, actin transport by the plus end can be observed also during the shrinking phase of a transporting microtubule.

Although the actin transport mechanism has been observed in a quasi 2D experiment, some preliminary experimental observations have also been performed in a droplet (Vendel et al., Methods in Molecular Biology, in press). Figure 5.4 shows that, even in the three dimensional case, some short transport events have 

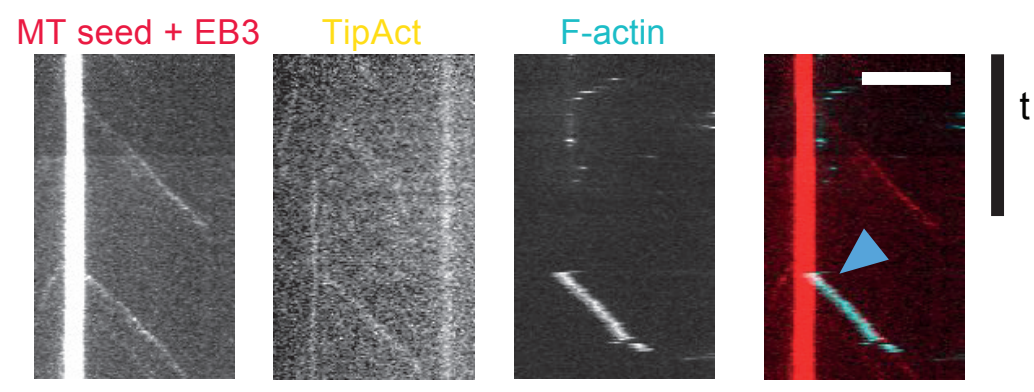

Figure 5.3: Transport of an actin filament by the plus end of a microtubule in presence of TipAct. Image taken from (Alkemade et al., in preparation).

A
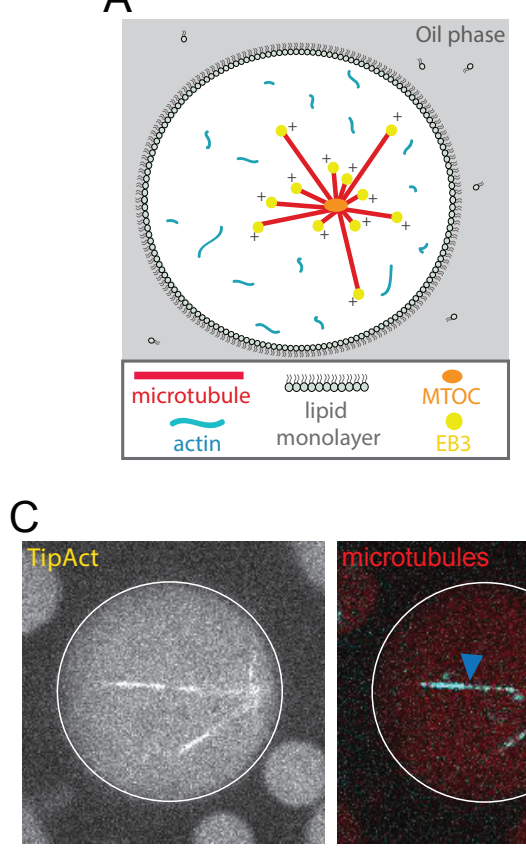

B

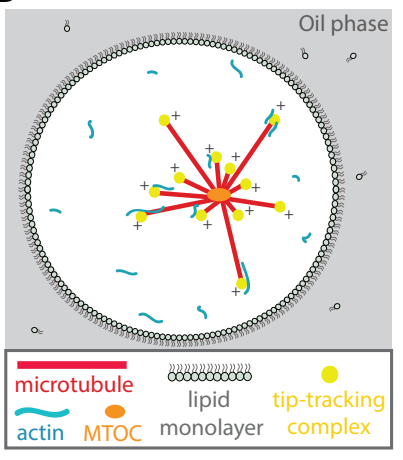

D
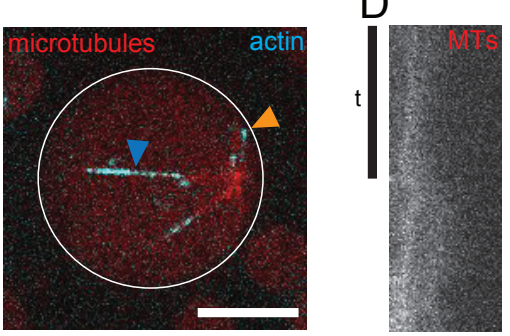

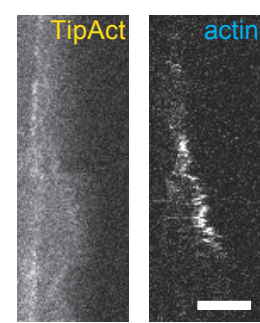

Figure 5.4: Microtubules and actin interacting in a three dimensional droplet. Schematic of a droplet containing a MTOC with several microtubules nucleating from it (aster) and diffusing actin, (A) without cytolinkers and (B) with cytolinkers. (C) Co-alignment of actin filaments to microtubules (blue arrow) in presence of cytolinkers. The orange arrow indicates the location of a short event of actin transport by the growing plus end of the microtubule shown in (D). Image taken from (Vendel et al., Methods in Molecular Biology, in press). 
been recorded, providing a proof of concept that actin transport by microtubules via cytolinkers is possible also in a three dimensional environment like, for example, a cell.

\subsection{The model: dynamic microtubules and diffusing actin}

So far no theoretical studies about the dynamics of microtubules and actin when they interact with each other have been performed. Therefore, here we explore the possible consequences of this interaction as regards the spatial organization of actin and microtubules in a confinement. In the remaining part of the chapter, we are interested in making predictions about how microtubules and actin mutually influence when they interact. Given that our interest also lies in the actin transport mechanism, we base our model on the experiments performed with TipAct. Finally, even though transport in three dimensions has not yet been incontrovertibly observed, here we study the system of actin and microtubules in a three dimensional environment. Nevertheless, some of the results obtained can be easily generalized to fewer dimensions.

\section{The model}

The model is once again based on the Dogterom-Leibler model also discussed in previous chapters, and consists of $M$ microtubules undergoing dynamic instability in an homogeneous 3-dimensional sphere of radius $R$, interacting with $A$ actin filaments diffusing with diffusion constant $D$. As we are interested in studying the interaction between actin and microtubules, and in particular in the transport of actin by the plus end of microtubules, in our model we ignore the polymerization and depolymerization of the actin filaments and we model them as dimensionless particles.

All microtubules are isotropically nucleated at position $\mathbf{x}=0$ in the growing state, radially growing with speed $v^{+}$. Besides the general properties of the dynamics of microtubules of Dogterom-Leibler model - i.e. alternation of growth and shrinkage due to rescues and catastrophes, every time the plus end of a microtubule is within the range of interaction $s$ of an actin filament, the microtubule tip and the filament can interact and bind together with binding rate $r_{b}$. The filament is then transported by the microtubule plus end towards the surface of the cell, with bound growing speed (or transport speed) $v^{b}<v^{+}$, accordingly to the experimental measurements. When bound together, microtubules and actin filaments can unbind in three distinct ways: i) they detach with constant unbinding 
rate $r_{u}$, ii) the microtubule undergoes a catastrophe and releases the particle with catastrophe rate $r_{c}$, iii) or a microtubule hits the surface of the cell, and it is reflected back in the shrinking state after releasing the actin. Microtubules are also subject to sudden re-nucleation as soon as they completely depolymerize, i.e. we impose reflective boundary conditions at $\mathbf{x}=0$ for the microtubules. Finally, actin filaments are completely reflected when they touch the boundary, or in other words, when they are at position $|\mathbf{x}| \equiv x=R$, see Figure 5.5.
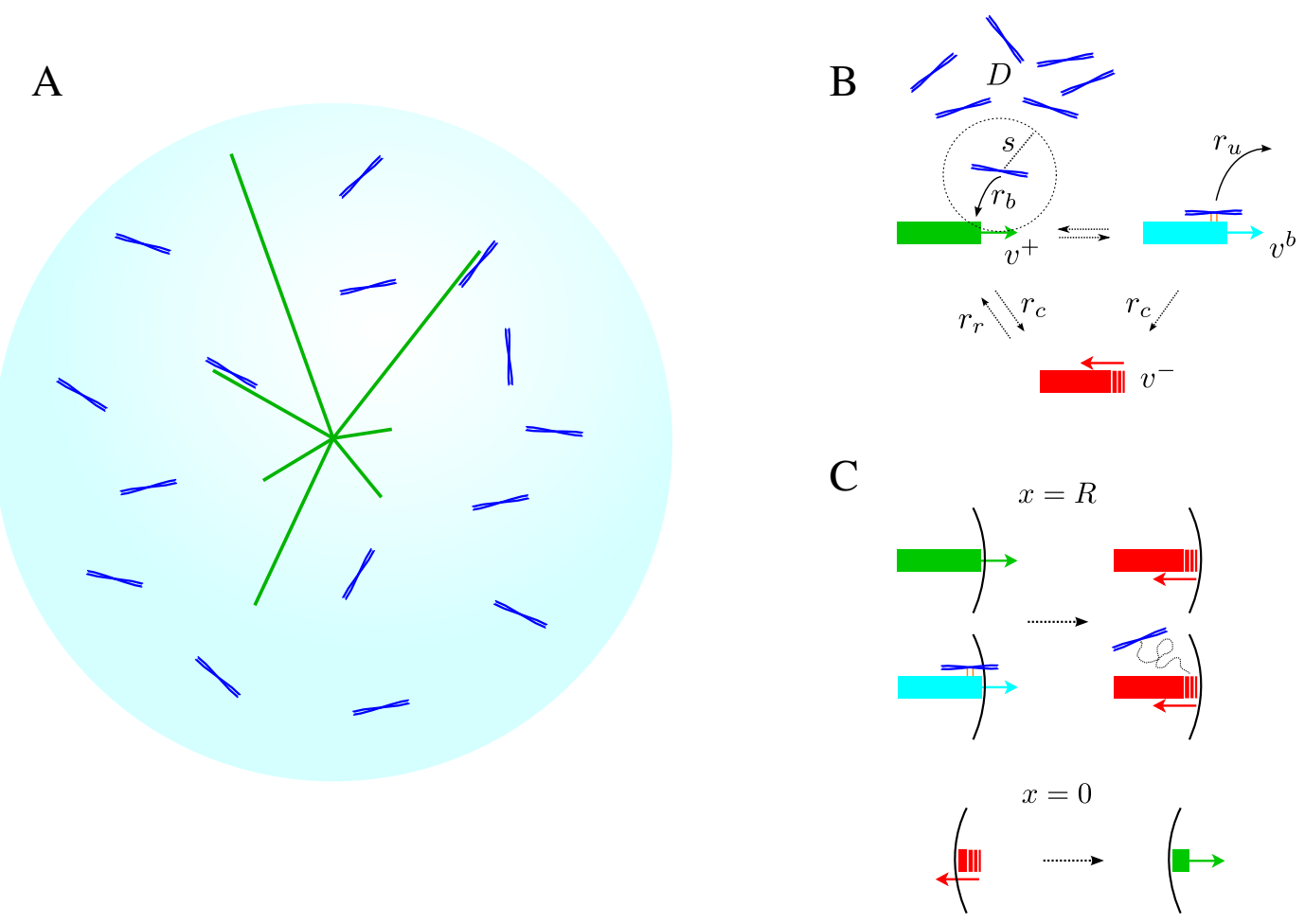

Figure 5.5: Schematic of the model. (A) Microtubules (green lines) undergoing dynamic instability and actin (blue spirals) diffusing in a 3-dimensional sphere. (B) Interaction between actin and microtubules. (C) Boundary conditions at the surface of the cell and at the centre.

\section{Dynamic equations}

As actin binds to microtubule plus ends with rate $r_{b}$ within a certain range of interaction $s$, here we assume that the actual interaction process is much faster than both the diffusion of the actin and the growth of a microtubule. Moreover, we assume that $s$ is small enough that the density distribution of growing microtubule 
plus ends is approximately constant in it. Therefore, if $m^{+}(t, \mathbf{x})$ is the distribution of free growing microtubules, and $a(t, \mathbf{x})$ the distribution of free actin filaments, the overall interaction rate is given by

$$
r_{b} s m^{+}(t, \mathbf{x}) a(t, \mathbf{x}) .
$$

Then, if we also introduce the distributions of the plus end of shrinking microtubules $m^{-}(t, \mathbf{x})$ and of the plus end of microtubules bound to actin $b(t, \mathbf{x})$, the dynamic equations that describe the system are

$$
\begin{gathered}
4 \pi x^{2} \frac{\partial m^{+}(t, \mathbf{x})}{\partial t}=-\mathbf{v}^{+} \cdot \nabla 4 \pi x^{2} m^{+}(t, \mathbf{x})-r_{c} 4 \pi x^{2} m^{+}(t, \mathbf{x}) \\
-r_{b} s 4 \pi x^{2} m^{+}(t, \mathbf{x}) a(t, \mathbf{x})+r_{u} 4 \pi x^{2} b(t, \mathbf{x})+r_{r} 4 \pi x^{2} m^{-}(t, \mathbf{x}), \\
4 \pi x^{2} \frac{\partial m^{-}(t, \mathbf{x})}{\partial t}=\mathbf{v}^{-} \cdot \nabla 4 \pi x^{2} m^{-}(t, \mathbf{x})+r_{c} 4 \pi x^{2}\left[m^{+}(t, \mathbf{x})+b(t, \mathbf{x})\right] \\
-r_{r} 4 \pi x^{2} m^{-}(t, \mathbf{x}), \\
\quad+r_{b} s 4 \pi x^{2} m^{+}(t, \mathbf{x}) a(t, \mathbf{x}), \\
\frac{\partial \pi x^{2} \frac{\partial b(t, \mathbf{x})}{\partial t}=-\mathbf{v}^{\mathbf{b}} \cdot \nabla 4 \pi x^{2} b(t, \mathbf{x})-\left(r_{c}+r_{u}\right) 4 \pi x^{2} b(t, \mathbf{x})}{\partial t}=D \nabla^{2} a(t, \mathbf{x})-r_{b} s m^{+}(t, \mathbf{x}) a(t, \mathbf{x})+\left(r_{u}+r_{c}\right) b(t, \mathbf{x}) .
\end{gathered}
$$

The first three equations are transport equations for the radial distributions ${ }^{1}$ of the plus end of growing microtubules, shrinking microtubules, and bound microtubules. The last equation is a diffusion equation for the free actin particle with a decay due to the capture of actin filaments by microtubule tips, and a source due to the release of actin from the tip to the pool.

Since the system exhibits spherical symmetry we make use of spherical coordinates to study the system, i.e. $\mathbf{x}=(x, \theta, \phi)$. We also notice that, given our assumptions of homogeneity and isotropy, all quantities of the model only depend on the radial coordinate $x$. Furthermore, it has been shown by Govindan and Spillman (Govindan \& Spillman, Phys. Rev. E, 2004) that microtubules undergoing dynamic instability in a confined volume always reach a steady-state, regardless of the choice of the dynamic parameters. Hence, we reasonably assume that our system always reaches the steady-state, and we restrict the study of Eqs. (5.1-5.4)

\footnotetext{
${ }^{1}$ The radial distribution is the function that describes the density of probability at a certain distance from the origin. In the case of, e.g., plus end of growing microtubules in three dimensions, it is defined as $4 \pi x^{2} m^{+}(t, \mathbf{x})$.
} 
in such a situation. Finally, since in the experiments no rescues have been observed, we make the further assumption that $r_{r}=0$. In this way, once a microtubule undergoes a catastrophe, its fate is determined as it cannot be rescued. Therefore, as we did in Chapter 4 , we make the final assumption that $v^{-} \gg v^{+}$, i.e. as soon as a microtubule undergoes a catastrophe it suddenly depolymerizes, and then it nucleates again. In this way the number of microtubules in the shrinking state and hence the related distribution, drops to zero and Eq. (5.2) vanishes.

With all these assumptions, we can rewrite Eqs. (5.1-5.4) in the steady-state as

$$
\begin{aligned}
0=-v^{+} & \frac{d}{d x}\left[x^{2} m(x)\right]-r_{c} x^{2} m(x)-r_{b} s x^{2} m(x) a(x)+r_{u} x^{2} b(x), \\
0 & =-v^{b} \frac{d}{d x}\left[x^{2} b(x)\right]-\left(r_{c}+r_{u}\right) x^{2} b(x)+r_{b} s x^{2} m(x) a(x), \\
0 & =D \frac{1}{x^{2}} \frac{d}{d x}\left[x^{2} \frac{d}{d x} a(x)\right]-r_{b} s m(x) a(x)+\left(r_{u}+r_{c}\right) b(x),
\end{aligned}
$$

where $m(x) \equiv m^{+}(x)$. This set of ordinary differential equations is supplemented by the boundary conditions defined by the properties of the model. Indeed, the sudden renucleation of every microtubule that undergoes a catastrophe implies

$$
v^{+} m(0)=v^{+} m(R)+v^{b} b(R)+r_{c} M
$$

and

$$
b(0)=0 .
$$

The release of actin filaments from microtubules when they arrive bound to the cell surface, together with the reflective boundary condition for the free diffusing actin, instead, imply

$$
\left.D \nabla a(x) \cdot \widehat{\mathbf{x}}\right|_{x=R}=v^{b} b(R) .
$$

Besides the boundary conditions, conservation of probability implies a normalization condition for both actin filaments and microtubules

$$
\begin{gathered}
4 \pi \int_{0}^{R} d x x^{2}[m(x)+b(x)]=M \\
4 \pi \int_{0}^{R} d x x^{2}[a(x)+b(x)]=A .
\end{gathered}
$$




\section{Numerical solution}

Unfortunately, due to the presence of the coupling term $m(x) a(x)$ in all dynamic equations (5.5-5.7), it is not possible to decouple them in order to write a closed form equation for every species, and consequently, an analytical solution of the model seems to be non-reachable. For this reason, in this section, we solve the model with numerical techniques.

As we are dealing with one second order and two first order differential equations, we need four boundary and/or normalization conditions in order to find a solution. Conditions (5.8-5.12) are in principle five. However, condition (5.10) implies condition (5.9) through the set (5.5-5.7). Therefore, the number of independent boundary/normalization conditions is four.

An interesting technique to include normalization conditions in the search for the integration constants for differential equations, consists of substituting the probability distributions $m(x), b(x)$, and $a(x)$ with the respective cumulative distribution functions, defined as

$$
\begin{gathered}
M(x)=4 \pi \int_{0}^{x} d x^{\prime}{x^{\prime}}^{2} m\left(x^{\prime}\right), \\
B(x)=4 \pi \int_{0}^{x} d x^{\prime} x^{\prime 2} b\left(x^{\prime}\right), \\
A(x)=4 \pi \int_{0}^{x} d x^{\prime}{x^{\prime}}^{2} a\left(x^{\prime}\right),
\end{gathered}
$$

from which it follows that

$$
m(x)=\frac{1}{4 \pi x^{2}} \frac{d M(x)}{d x},
$$

and similarly for $B(x)$ and $A(x)$. With this substitution, Eqs. (5.5-5.12) become

$$
\begin{gathered}
0=-v^{+} M^{\prime \prime}(x)-r_{c} M^{\prime}(x)-r_{b} s \frac{M^{\prime}(x) A^{\prime}(x)}{4 \pi x^{2}}+r_{u} B^{\prime}(x) \\
0=-v^{b} B^{\prime \prime}(x)-\left(r_{c}+r_{u}\right) B^{\prime}(x)+r_{b} s \frac{M^{\prime}(x) A^{\prime}(x)}{4 \pi x^{2}} \\
0=D\left[A^{\prime \prime \prime}(x)-\frac{2}{x} A^{\prime \prime}(x)+\frac{2}{x^{2}} A^{\prime}(x)\right]-r_{b} s \frac{M^{\prime}(x) A^{\prime}(x)}{4 \pi x^{2}}+\left(r_{u}+r_{c}\right) B^{\prime}(x), \\
v^{+} M^{\prime}(0)=v^{+} M^{\prime}(R)+v^{b} B^{\prime}(R)+r_{c} M \\
B^{\prime}(0)=0
\end{gathered}
$$




$$
\begin{gathered}
D\left[A^{\prime \prime}(R)-\frac{2}{R} A^{\prime}(R)\right]=v^{b} B^{\prime}(R), \\
M(R)-M(0)+B(R)-B(0)=M, \\
A(R)-A(0)+B(R)-B(0)=A .
\end{gathered}
$$

Now, we can numerically solve the system. As it is now a seventh order set of differential equations and we only have five conditions, the numerical solution yields two unknown integration constants. However, given our interest in computing the original distributions

$$
\begin{aligned}
& m(x)=\frac{1}{4 \pi x^{2}} \frac{d M(x)}{d x} \\
& b(x)=\frac{1}{4 \pi x^{2}} \frac{d B(x)}{d x}
\end{aligned}
$$

and

$$
a(x)=\frac{1}{4 \pi x^{2}} \frac{d A(x)}{d x},
$$

the final derivation from the cumulative to the original cancels out the unknown integration constants. Figure 5.6 shows the numerical solution of the set of Eqs. (5.13-5.20), with the value for the model parameters taken from Table 5.1. We can observe that, in this case, the interaction plays a minimal role in changing the distribution of the free actin as a consequence of the transport mechanism, as the distribution is roughly uniform (Figure 5.6C). On the contrary, we can observe a peak in the distribution of bound microtubules very close to the nucleation point. Indeed, as $x \rightarrow 0$, the density of microtubule plus ends is very high, since all microtubules are nucleated at $x=0$. Furthermore, those that capture an actin filament reduce their growth speed from $v^{+}$to $v^{b}$, resulting in the creation of a trapping effect for the actin close to the centre of the cell. In addition, the diffusion coefficient is large enough to quickly redistribute the actin filaments that detach from microtubule plus ends all over the volume of the sphere, allowing the availability of a good amount of free actin in the central area of the cell, and therefore, their possible capture. Finally, due to the dilution of microtubule plus ends caused by their radial direction from the centre of the cell, we can observe that the distribution of microtubules - both growing and bound, very quickly decreases to low values, see Figure 5.6AB.

To test the hypothesis that the trapping mechanism is due to large $D$ and slow $v^{b}$, we numerically solve the set of Eqs. (5.13-5.20) for different values of these two parameters. Figure 5.7A shows that, by reducing the diffusion coefficient for the free actin, we can deplete the amount of free actin in the centre of the cell, as the actin filaments released closer to the surface do not redistribute fast. Figure 

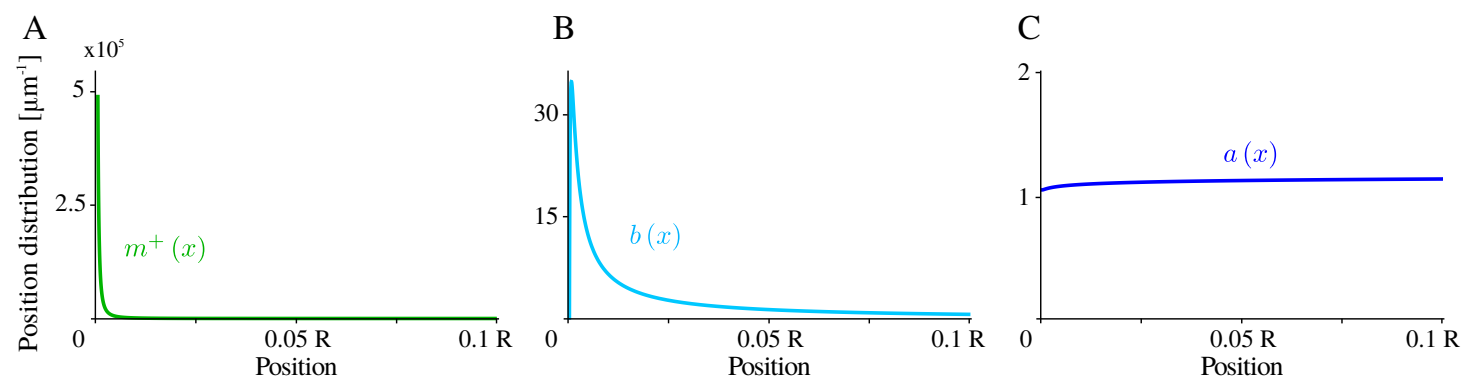

Figure 5.6: Distribution of the position of (A) growing microtubule plus ends, (B) bound microtubule plus ends, and $(\mathrm{C})$ actin filaments.
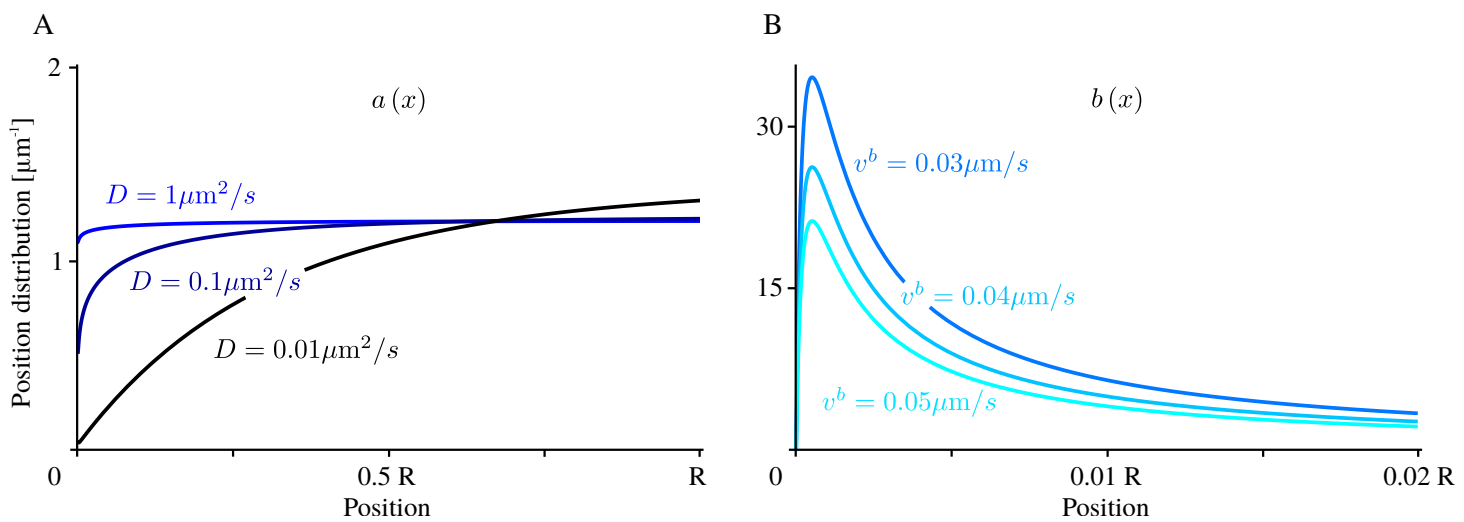

Figure 5.7: (A) Distribution of the position of actin filaments for different values of the diffusion coefficient $D$. (B) Distribution of the position of bound microtubule plus ends for different values of the bound growth speed $v^{b}$. Model parameters not explicitly tuned are taken from Table 5.1. 
Microtubule-based actin transport in a three dimensional confinement

\begin{tabular}{cccc}
\hline \hline Parameter & Description & Numerical value & Units \\
\hline$v^{+}$ & Free-growth speed & 0.05 & $\mu \mathrm{m} \mathrm{s}^{-1}$ \\
$v^{b}$ & Transport speed & 0.03 & $\mu \mathrm{m} \mathrm{s}^{-1}$ \\
$r_{c}$ & Catastrophe rate & 0.005 & $\mathrm{~s}^{-1}$ \\
$r_{b}$ & Binding rate & 0.8 & $\mathrm{~s}^{-1}$ \\
$s$ & Actin-microtubule interaction volume & 0.002 & $\mu \mathrm{m}^{3}$ \\
$r_{u}$ & Unbinding rate & 0.009 & $\mathrm{~s}^{-1}$ \\
$D$ & Free actin diffusion coefficient & 1 & $\mu \mathrm{m}^{2} \mathrm{~s}^{-1}$ \\
$R$ & Radius of the cell & 10 & $\mu \mathrm{m}$ \\
$M$ & Total number of microtubules & $10^{4}$ & - \\
$A$ & Total number of actin filaments & $5 \cdot 10^{4}$ & - \\
\hline
\end{tabular}

Table 5.1: Model parameters. The choice for the numerical values is in agreement with the experimental measurements of the same quantity (Alkemade et al., in preparation).

$5.7 \mathrm{~B}$, instead, shows that the increase of the transport speed $v^{b}$ up to $v^{+}$causes a reduction of the trapping effect, as the bound actin filaments are transported faster toward the outer area of the cell. Therefore, the combination of a slow diffusion and a fast transport can cause localization of actin at the cell surface rather than at its centre, see Figure 5.8. Figure 5.8 also exhibits another feature: even though in the slow diffusion and fast transport regime we can drive the actin filaments toward the surface of the cell, a fraction of them is always trapped at the centre, unless we further increase the transport speed to non-biologically realistic values. In Section 5.4 we will give an analytical explanation of this mechanism.

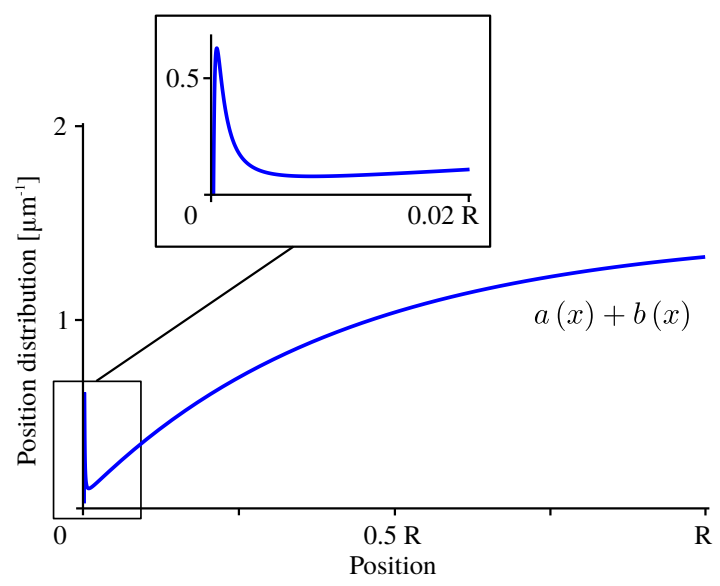

Figure 5.8: Total distribution $a(x)+b(x)$ of the actin filaments for slow diffusion and fast transport, i.e. $D=0.01 \mu \mathrm{m}^{2} / s$ and $v^{b}=0.05 \mu \mathrm{m} / s$. 


\subsection{Fast diffusion limit: an analytical solution}

In this section we study the actin-microtubule system under the assumption that the only effect of their interaction is to change the dynamic properties of microtubules, without any influence on the distribution of the free actin filaments. Practically, assuming that the free actin distribution is not influenced by microtubule dynamics means that the diffusion coefficient for actin filaments is $D \rightarrow \infty$, i.e. actin is always homogeneously distributed in the whole cell. We also assume that the amount of free actin filaments is available in abundance. That allows us to keep the binding rate constant or, in other words, to make the substitution $r_{b} s a(x) \rightarrow r_{b}$. This change makes Eqs. (5.5-5.7) be decoupleable, and therefore, suitable to be approached analytically.

\section{Dynamic equations}

Here, in contrast with the previous section, we work in the limit in which after a catastrophe, shrinking microtubules need a finite amount of time to completely depolymerize. Moreover, we assume that nucleation does not occur just after the complete depolymerization, but a new microtubule is nucleated with pro capita nucleation rate $r_{n}$.

Given the radial symmetry of the problem and the absence of inhomogeneity in the actin concentration, we limit the discussion to the radial part of the probability distributions of the plus ends. In other words, we face the problem as if it was 1-dimensional. Furthermore, as every microtubule is nucleated at position $x=0$, we can identify the position of the plus end with the length of the microtubule.

In this case, the steady-state version of Eqs. (5.1-5.4) are

$$
\begin{gathered}
0=-v^{+} \frac{d}{d x} m^{+}(x)-r_{c} m^{+}(x)-r_{b} m^{+}(x)+r_{u} b(x), \\
0=-v^{b} \frac{d}{d x} b(x)-\left(r_{c}+r_{u}\right) b(x)+r_{b} m^{+}(x), \\
0=v^{-} \frac{d}{d x} m^{-}(x)+r_{c}\left[m^{+}(x)+b(x)\right] .
\end{gathered}
$$

Since all microtubules are nucleated in the growing state, at $x=0$ the boundary conditions are

$$
\begin{gathered}
v^{+} m^{+}(0)=r_{n} M_{n}, \\
b(0)=0 \\
v^{-} m^{-}(0)=v^{+} m^{+}(0)+v^{b} b(0)=r_{n} M_{n},
\end{gathered}
$$


where $M_{n}$ is the number of microtubules in the dormant state, i.e. microtubules completely depolymerized and waiting to be re-nucleated, $M_{n}=M-\left(M_{0}^{+}+M_{0}^{-}+B_{0}\right)$. At $x=R$, instead, the boundary condition is

$$
v^{-} m^{-}(R)=v^{+} m^{+}(R)+v^{b} b(R) .
$$

For the sake of simplicity in the derivation, we make the following nondimensionalizations:

- microtubule length

$$
\lambda=\frac{r_{c}+r_{b}}{v^{+}} x
$$

- microtubule length distribution in the growing/shrinking state

$$
\mu^{ \pm}(\lambda)=\frac{v^{+}}{r_{n}} m^{ \pm}(x)
$$

- microtubule length distribution in the bound state

$$
\beta(\lambda)=\frac{v^{+}}{r_{n}} b(x)
$$

- unbinding rate

$$
\omega=\frac{r_{u}}{r_{c}+r_{b}},
$$

- catastrophe rate

$$
\rho=\frac{r_{c}}{r_{c}+r_{b}},
$$

- growing/shrinking speed

$$
\nu^{ \pm}=\frac{v^{ \pm}}{v^{+}}
$$

- bound or transport speed

$$
\nu^{b}=\frac{v^{b}}{v^{+}}
$$

- re-scaled number of dormant microtubules

$$
\mathcal{M}_{n}=\frac{r_{c}+r_{b}}{r_{n}} M_{n},
$$


and therefore, we rewrite Eqs. (5.21), (5.22), and (5.23) as

$$
\begin{gathered}
\frac{d}{d \lambda} \mu^{+}(\lambda)=-\mu^{+}(\lambda)+\omega \beta(\lambda), \\
\nu^{b} \frac{d}{d \lambda} \beta(\lambda)=-(\rho+\omega) \beta(\lambda)+(1-\rho) \mu^{+}(\lambda), \\
-\nu^{-} \frac{d}{d \lambda} \mu^{-}(\lambda)=\rho\left[\mu^{+}(\lambda)+\beta(\lambda)\right],
\end{gathered}
$$

supported by the boundary conditions

$$
\begin{gathered}
\mu^{+}(0)=\mathcal{M}_{n}, \\
\beta(0)=0, \\
\mu^{-}(0)=\frac{\mathcal{M}_{n}}{\nu^{-}},
\end{gathered}
$$

and

$$
\mu^{+}(\Lambda)+\nu^{b} \beta(\Lambda)=\nu^{-} \mu^{-}(\Lambda)
$$

\section{Steady-state solution}

By combining Eqs. (5.24) and (5.25) together, we can split them to obtain two second order linear differential equations

$$
\frac{d^{2}}{d \lambda^{2}} \pi(\lambda)+\left(1+\frac{\rho+\omega}{\nu^{b}}\right) \frac{d}{d \lambda} \pi(\lambda)+\frac{\rho(1+\omega)}{\nu^{b}} \pi(\lambda)=0
$$

where $\pi=\mu^{+}, \beta$. Equation (5.31) has different solutions depending on the sign of $\Delta^{2} \equiv\left(1+\frac{\rho+\omega}{\nu^{b}}\right)^{2}-4 \frac{\rho(1+\omega)}{\nu^{b}}$. However, given that from $v^{b}<v^{+}$it follows that $\nu^{b}<1$, we can show that

$$
\begin{aligned}
\Delta^{2} & =\frac{\left(\rho-\nu^{b}\right)^{2}+\omega\left(\omega+2 \nu^{b}-4 \nu^{b} \rho+2 \rho\right)}{\left(\nu^{b}\right)^{2}} \\
& =\frac{\left(\rho-\nu^{b}\right)^{2}+\omega\left(\omega+2 \nu^{b}-4 \nu^{b} \rho+4 \rho-2 \rho\right)}{\left(\nu^{b}\right)^{2}} \\
& =\frac{\left(\rho-\nu^{b}\right)^{2}+\omega\left(\omega+2 \nu^{b}+4 \rho\left(1-\nu^{b}\right)-2 \rho\right)}{\left(\nu^{b}\right)^{2}}>\frac{\left(\rho-\nu^{b}\right)^{2}+\omega\left(\omega+2 \nu^{b}-2 \rho\right)}{\left(\nu^{b}\right)^{2}}
\end{aligned}
$$




$$
=\frac{\left(\rho-\nu^{b}\right)^{2}+\omega^{2}-2 \omega\left(\rho-\nu^{b}\right)}{\left(\nu^{b}\right)^{2}}=\frac{\left(\rho-\nu^{b}-\omega\right)^{2}}{\left(\nu^{b}\right)^{2}} \geq 0,
$$

and hence the solution of Eq. (5.31) is

$$
\pi(\lambda)=e^{-\frac{1}{2}\left(1+\frac{\rho+\omega}{\nu}\right) \lambda}\left[c_{1, \pi} e^{\frac{1}{2} \Delta \lambda}+c_{2, \pi} e^{-\frac{1}{2} \Delta \lambda}\right] .
$$

By plugging this equation in Eq. (5.26), we can find the expression for $\mu^{-}(\lambda)$ as well. Another way to obtain $\mu^{-}(\lambda)$ is to sum Eqs. (5.24), (5.25), and (5.26) together. By doing this we obtain

$$
\frac{d}{d \lambda}\left[\mu^{+}(\lambda)+\nu^{b} \beta(\lambda)-\nu^{-} \mu^{-}(\lambda)\right]=0,
$$

that, together with the conditions at $\lambda \rightarrow \infty$, i.e. $\lim _{\lambda \rightarrow \infty} \mu^{\sigma}(\lambda)=0$, implies

$$
\mu^{+}(\lambda)+\nu^{b} \beta(\lambda)=\nu^{-} \mu^{-}(\lambda), \quad \forall \lambda \in[0, \infty) .
$$

Consequently, $\mu^{-}(\lambda)$ is a linear combination of the other two distributions, and we can summarize all of them as

$$
\pi(\lambda)=e^{-\frac{1}{2}\left(1+\frac{\rho+\omega}{\nu}\right) \lambda}\left[c_{1, \pi} e^{\frac{1}{2} \Delta \lambda}+c_{2, \pi} e^{-\frac{1}{2} \Delta \lambda}\right],
$$

where here $\pi=\mu^{ \pm}, \beta$, and

$$
c_{j,-}=\frac{c_{j,+}+\nu^{b} c_{j, b}}{\nu^{-}}
$$

Unfortunately the four boundary conditions (5.27-5.30) are linearly dependent. Thus we cannot find all four independent integration coefficients $c_{1,+/ b}$ and $c_{2,+/ b}$. However, if we plug Eq. (5.34) for $\mu^{+}$and $\beta$ in Eq. (5.26), we can find a second expression for $\mu^{-}$, namely

$$
\begin{gathered}
\mu^{-}(\lambda)=\frac{\mathcal{M}_{n}}{\nu^{-}}-\frac{2 \rho \nu^{b}}{\nu^{-}}\left(\frac{c_{1,+}+c_{1, b}}{\nu^{b}(1-\Delta)+\rho+\omega}+\frac{c_{2,+}+c_{2, b}}{\nu^{b}(1+\Delta)+\rho+\omega}\right) \\
+e^{-\frac{1}{2}\left(1+\frac{\rho+\omega}{\nu^{b}}\right) \lambda}\left[\frac{2 \rho \nu^{b}\left(c_{1,+}+c_{1, b}\right)}{\nu^{-}\left(\nu^{b}(1-\Delta)+\rho+\omega\right)} e^{\frac{1}{2} \Delta \lambda}\right. \\
\left.\quad+\frac{2 \rho \nu^{b}\left(c_{2,+}+c_{2, b}\right)}{\nu^{-}\left(\nu^{b}(1+\Delta)+\rho+\omega\right)} e^{-\frac{1}{2} \Delta \lambda}\right] .
\end{gathered}
$$

The comparison between Eq. (5.34) for $\mu^{-}$and (5.36), together with the observation that

$$
a+b e^{x}=c e^{x} \quad \forall x \quad \Rightarrow \quad a=0 \wedge b=c,
$$


imply that

$$
\begin{gathered}
c_{1,+}=\mathcal{M}_{n} \frac{\Delta-1+\frac{\rho+\omega}{\nu^{b}}}{2 \Delta}, \\
c_{2,+}=\mathcal{M}_{n}-c_{1,+}=\mathcal{M}_{n} \frac{\Delta+1-\frac{\rho+\omega}{\nu^{b}}}{2 \Delta}, \\
c_{1, b}=\mathcal{M}_{n} \frac{1-\rho}{\nu^{b} \Delta}, \\
c_{2, b}=-c_{1, b}=-\mathcal{M}_{n} \frac{1-\rho}{\nu^{b} \Delta}, \\
c_{1,-}=\frac{c_{1,+}+\nu^{b} c_{1, b}}{\nu^{-}}=\mathcal{M}_{n} \frac{\Delta+\frac{\rho+\omega}{\nu^{b}}-2 \rho+1}{2 \nu^{-} \Delta}, \\
c_{2,-}=\frac{c_{2,+}+\nu^{b} c_{2, b}}{\nu^{-}}=\mathcal{M}_{n} \frac{\Delta-\frac{\rho+\omega}{\nu^{b}}+2 \rho-1}{2 \nu^{-} \Delta} .
\end{gathered}
$$

Finally, from

$$
\mathcal{M}_{n}=\mathcal{M}-\left(\mathcal{M}_{0}^{+}+\mathcal{M}_{0}^{-}+\mathcal{B}_{0}\right)=\mathcal{M}-\int_{0}^{\Lambda} d \lambda\left[\mu^{+}(\lambda)+\mu^{-}(\lambda)+\beta(\lambda)\right],
$$

we can calculate the number of dormant microtubules

$$
\begin{aligned}
& \mathcal{M}_{n}=\{1 \\
& +\frac{r_{n}}{r_{c}+r_{b}}\left[\frac{1-\rho}{\nu^{b} \Delta}\left(1+\frac{\nu^{b}}{\nu^{-}}\right)+\frac{\Delta-1+\frac{\rho+\omega}{\nu^{b}}}{2 \Delta}\left(1+\frac{1}{\nu^{-}}\right)\right] \\
& \times \frac{1-e^{-\frac{1}{2}\left(1+\frac{\rho+\omega}{\nu^{b}}-\Delta\right) \Lambda}}{\frac{1}{2}\left(1+\frac{\rho+\omega}{\nu^{b}}-\Delta\right)} \\
& +\frac{r_{n}}{r_{c}+r_{b}}\left[1+\frac{1}{\nu^{-}}-\frac{1-\rho}{\nu^{b} \Delta}\left(1+\frac{\nu^{b}}{\nu^{-}}\right)-\frac{\Delta-1+\frac{\rho+\omega}{\nu^{b}}}{2 \Delta}\left(1+\frac{1}{\nu^{-}}\right)\right] \\
& \left.\times \frac{1-e^{-\frac{1}{2}\left(1+\frac{\rho+\omega}{\nu^{b}}+\Delta\right) \Lambda}}{\frac{1}{2}\left(1+\frac{\rho+\omega}{\nu^{b}}+\Delta\right)}\right\}^{-1} \text {. }
\end{aligned}
$$

Figure 5.9A shows the probability distributions for microtubule length. We can observe that $\beta(\lambda)$ steeply increases to reach a peak that defines the length scale for 
microtubules to freely grow before binding to actin. Figure 5.9B, instead, compares the total length distribution with those of the Dogterom-Leibler model. We can observe that the change in the dynamic parameters implies a change in the shape of the steady-state length distribution with respect to the basic model. In Chapter 2 we have already observed such an effect. Here, however, we are interested in discussing the causes of this change. The position $\lambda_{\text {peak }}$ of such a peak can be calculated via $0=\frac{d \beta}{d \lambda}$, and it is

$$
\lambda_{\text {peak }}=\frac{1}{\Delta} \log \frac{1+\frac{\rho+\omega}{\nu^{b}}+\Delta}{1+\frac{\rho+\omega}{\nu^{b}}-\Delta} .
$$

Figure 5.10 shows how the position of the peak changes when we change, in turn, $\rho, \omega$, and $\nu^{b}$. We observe that, besides the case in which $\rho \rightarrow 0$, the position of the peak in the cell is shifted to shorter distance, less than halfway from the nucleation point to the cell surface. This means that the only strategy to strongly control the position of the peak is to considerably lower the catastrophe rate $\rho$. Intuitively, we can think that in the case of no catastrophe, every microtubule grows until it reaches the surface, and therefore it generates a peak in the distribution of the bound microtubules at $\lambda=\Lambda$. Except for this limit, the localization of the peak in the central part of the cell confirms the existence of the trapping effect for actin filaments already observed in Section 5.2, due to the interaction with microtubules. In other words, in the fast diffusion limit, it is not possible to localize actin filaments at the cell surface, since they are trapped by the microtubules at $\lambda_{\text {peak }}$.

If we add Eq. (5.24) to Eq. (5.25), we obtain

$$
\frac{d}{d \lambda}\left[\mu(\lambda)+\nu^{b} \beta(\lambda)\right]=-\rho[\mu(\lambda)+\beta(\lambda)],
$$

which means that if we set $\nu^{b}=1$, which corresponds to $v^{+}=v^{b}$, the solution of the last differential equation for $\mu(\lambda)+\beta(\lambda)$ is

$$
\mu(\lambda)+\beta(\lambda)=\mathcal{M}_{n} e^{-\rho \lambda}
$$

i.e. the steady-state distribution for microtubule length in Dogterom-Leibler model. Eq. (5.40) highlights an important feature of the model: the changed length distribution for the microtubules does not come from the interaction with the actin per se, but it is a consequence of the co-existence of two different states for growing microtubules with different speeds. Notice that this argument holds in the case of different catastrophe rates but same growing speeds as well. 

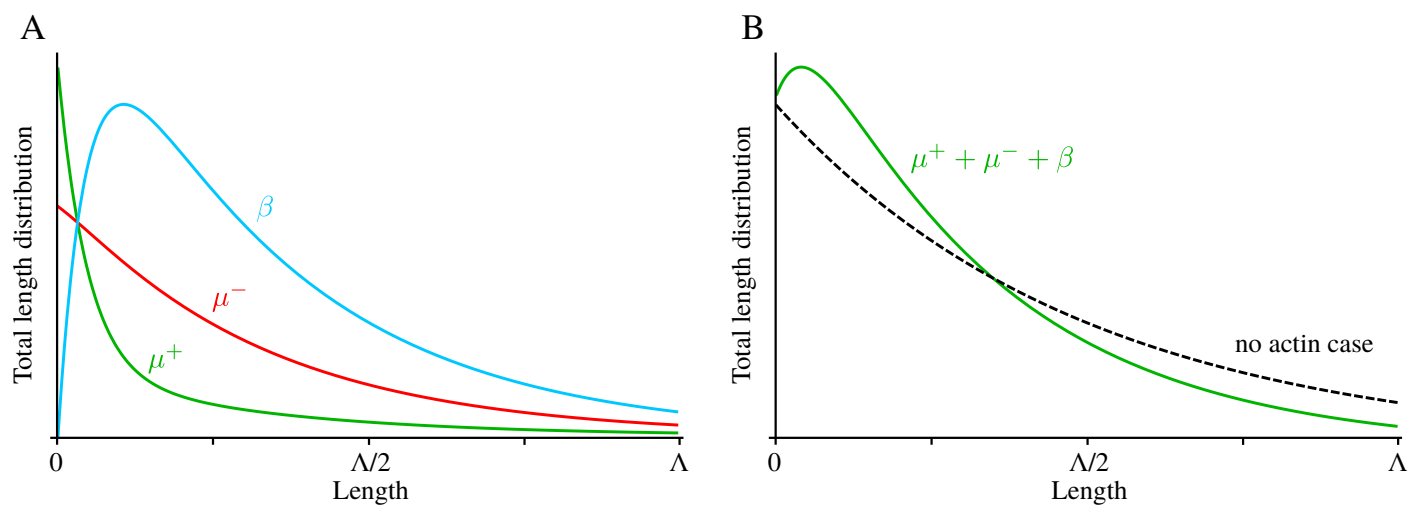

Figure 5.9: (A) Distribution of microtubule length for growing (green), shrinking (red), and bound microtubules (turquoise). (B) Overall length distribution (green straight line) compared with the length distribution of Dogterom-Leibler model (black dashed line).
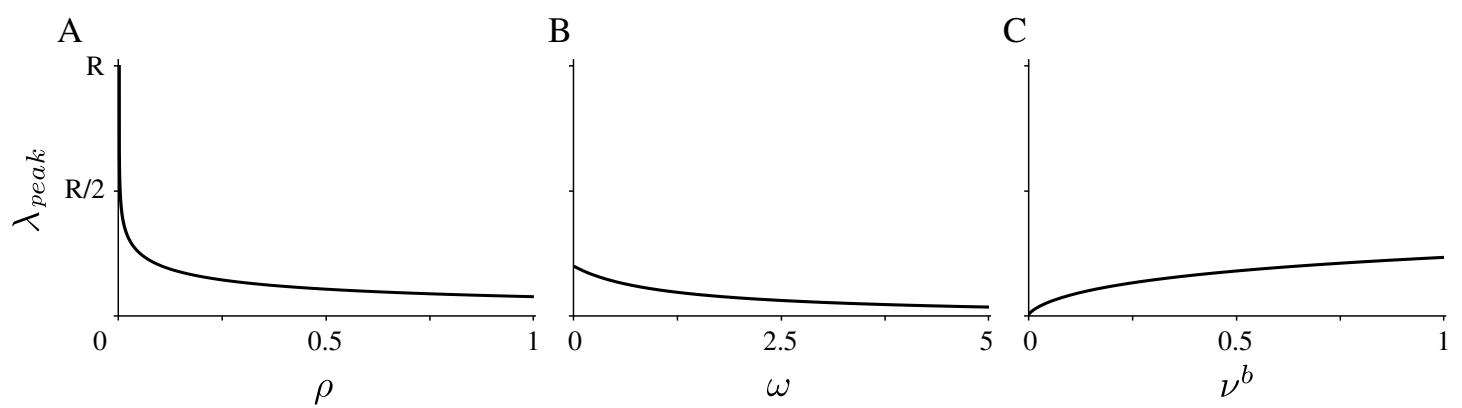

Figure 5.10: Location of the peak of $\beta(\lambda)$ as a function of (A) $\rho,(\mathrm{B}) \omega$, and (C) $\nu^{b}$. While $\rho$ and $\nu^{b}$ are comprises between 0 and 1 as a consequence of their definitions, $\omega$ does not have any a priori upper bound. 


\section{Probability to reach the surface}

Next to the problem of finding the length distribution of microtubules, an interesting question to address is what is the probability $\Pi_{\sigma \tau}\left(\Lambda \mid \lambda_{0}\right)$ that a microtubule initially at $\lambda=\lambda_{0}$ and in state $\tau$, reaches the surface at $\lambda=\Lambda$ in the state $\sigma$. Given the absence of rescues in the model, this problem has a trivial solution when the initial state is the shrinking state, i.e.

$$
\Pi_{\sigma-}\left(\Lambda \mid \lambda_{0}\right)=0
$$

In order to calculate $\Pi_{\sigma \tau}\left(\Lambda \mid \lambda_{0}\right)$ when $\tau=+/ b$, we first introduce the survival probability $S_{\sigma}\left(t \mid t_{0}\right)$ of a microtubule in the state $\sigma$, as the probability that a microtubule initially in the state $\sigma$ at time $t_{0}$ is still in the state $\sigma$ at time $t$. Consider a microtubule initially in the growing state. We can show (Mulder, Phys. Rev. E, 2012) that its survival probability in the growing state obeys the backward Kolmogorov equation as it evolves as

$$
\frac{d}{d t} S_{+}\left(t \mid t_{0}\right)=-r_{c} S_{+}\left(t \mid t_{0}\right)-r_{b} S_{+}\left(t \mid t_{0}\right)
$$

with initial condition $S_{+}\left(t_{0} \mid t_{0}\right)=1$. However, since the growing speed of microtubules is constant, we can make the substitution $t=\frac{x}{v^{+}}$and $\frac{d}{d t}=v^{+} \frac{d}{d x}$. Therefore, with the non-dimensionalized notation, our differential equation becomes

$$
\frac{d}{d \lambda} S_{+}\left(\lambda \mid \lambda_{0}\right)=-\rho S_{+}\left(\lambda \mid \lambda_{0}\right)-(1-\rho) S_{+}\left(\lambda \mid \lambda_{0}\right)=-S_{+}\left(\lambda \mid \lambda_{0}\right),
$$

the solution of which is

$$
S_{+}\left(\lambda \mid \lambda_{0}\right)=e^{-\left(\lambda-\lambda_{0}\right)} .
$$

Similarly, we can compute the survival probability in the bound state as

$$
S_{b}\left(\lambda \mid \lambda_{0}\right)=e^{-\frac{\rho+\omega}{\nu^{b}}\left(\lambda-\lambda_{0}\right)} .
$$

We call these two functions spatial survival probabilities in the growing/bound state, respectively. Given that in both cases a microtubule can leave its original state growing/bound, for two possible distinct states - bound/growing and shrinking, and because of the homogeneity of the system, we can factorize both $S_{+}\left(\lambda \mid \lambda_{0}\right)$ and $S_{b}\left(\lambda \mid \lambda_{0}\right)$ as

$$
S_{+}\left(\lambda \mid \lambda_{0}\right)=e^{-\rho\left(\lambda-\lambda_{0}\right)} e^{-(1-\rho)\left(\lambda-\lambda_{0}\right)} \equiv T_{-+}\left(\lambda \mid \lambda_{0}\right) T_{b+}\left(\lambda \mid \lambda_{0}\right),
$$

and

$$
S_{b}\left(\lambda \mid \lambda_{0}\right)=e^{-\frac{\rho}{\nu^{b}}\left(\lambda-\lambda_{0}\right)} e^{-\frac{\omega}{\nu^{b}}\left(\lambda-\lambda_{0}\right)} \equiv T_{-b}\left(\lambda \mid \lambda_{0}\right) T_{+b}\left(\lambda \mid \lambda_{0}\right),
$$


where $T_{\sigma \tau}\left(\lambda \mid \lambda_{0}\right)$ is the spatial partial survival probability for a microtubule to survive in the state $\tau$ without switching specifically to the state $\sigma$.

Knowing the spatial survival probabilities, we can define the function $Q_{\sigma \tau}\left(\lambda \mid \lambda_{0}\right)$ as the space dependent rate where a microtubule leaves the state $\tau$ to enter the state $\sigma$. Such function is defined as

$$
Q_{\sigma \tau}\left(\lambda \mid \lambda_{0}\right)=\prod_{\eta \neq \sigma} T_{\eta \tau}\left(\lambda \mid \lambda_{0}\right)\left[-\frac{d}{d \lambda} T_{\sigma \tau}\left(\lambda \mid \lambda_{0}\right)\right]
$$

Intuitively, we can think that a microtubule initially in the growing state with length $\lambda_{0}$ reaches the length $\lambda$ again in the growing state in two different ways: either it never leaves the growing state, or it binds and unbinds to actin any number of times, as long as from a certain moment when it is in the growing state it never leaves it again. Therefore, we can finally write an expression for the probabilities of reaching $\lambda$ in the growing state as

$$
\begin{gathered}
\Pi_{++}\left(\lambda \mid \lambda_{0}\right)=S_{+}\left(\lambda \mid \lambda_{0}\right)+\int_{\lambda_{0}}^{\lambda} d \lambda^{\prime} Q_{b+}\left(\lambda^{\prime} \mid \lambda_{0}\right) \Pi_{+b}\left(\lambda \mid \lambda^{\prime}\right) \\
\Pi_{+b}\left(\lambda \mid \lambda_{0}\right)=\int_{\lambda_{0}}^{\lambda} d \lambda^{\prime} Q_{+b}\left(\lambda^{\prime} \mid \lambda_{0}\right) \Pi_{++}\left(\lambda \mid \lambda^{\prime}\right) .
\end{gathered}
$$

If we combine Eqs. (5.47) and (5.48) we can split them to obtain:

$$
\begin{aligned}
\Pi_{++}\left(\lambda \mid \lambda_{0}\right)= & S_{+}\left(\lambda \mid \lambda_{0}\right) \\
& +\frac{\omega(1-\rho)}{\nu^{b}} \int_{\lambda_{0}}^{\lambda} d \lambda^{\prime} S_{+}\left(\lambda^{\prime} \mid \lambda_{0}\right) \int_{\lambda^{\prime}}^{\lambda} d \lambda^{\prime \prime} S_{b}\left(\lambda^{\prime \prime} \mid \lambda^{\prime}\right) \Pi_{++}\left(\lambda \mid \lambda^{\prime \prime}\right) \\
\Pi_{+b}\left(\lambda \mid \lambda_{0}\right)= & \frac{\omega}{\nu^{b}} \frac{1}{1+\frac{\rho+\omega}{\nu^{b}}}\left[1-S_{b}\left(\lambda \mid \lambda_{0}\right) S_{+}\left(\lambda \mid \lambda_{0}\right)\right] \\
& +\frac{\omega(1-\rho)}{\nu^{b}} \int_{\lambda_{0}}^{\lambda} d \lambda^{\prime} S_{b}\left(\lambda^{\prime} \mid \lambda_{0}\right) \int_{\lambda^{\prime}}^{\lambda} d \lambda^{\prime \prime} S_{+}\left(\lambda^{\prime \prime} \mid \lambda^{\prime}\right) \Pi_{+b}\left(\lambda \mid \lambda^{\prime \prime}\right)
\end{aligned}
$$

We now focus on the resolution of Eq. (5.49). First of all, we notice that because of the homogeneity of the system, all probabilities involved are functions of just the distance between the starting and the final positions. Hence, by making use of this property and by changing integration variables as $\lambda \rightarrow \lambda+\lambda_{0}, \lambda^{\prime} \rightarrow \lambda^{\prime}+\lambda_{0}$, and $\lambda^{\prime \prime} \rightarrow \lambda^{\prime \prime}+\lambda_{0}$, we can rewrite Eq. (5.49)

$$
\Pi_{++}(\lambda)=S_{+}(\lambda)+\frac{\omega(1-\rho)}{\nu^{b}} \int_{0}^{\lambda} d \lambda^{\prime} S_{+}\left(\lambda^{\prime}\right) \int_{\lambda^{\prime}}^{\lambda} d \lambda^{\prime \prime} S_{b}\left(\lambda^{\prime \prime}-\lambda^{\prime}\right) \Pi_{++}\left(\lambda-\lambda^{\prime \prime}\right) .
$$


If we Laplace transform last equation we obtain

$$
\widehat{\Pi}_{++}(s)=\int_{0}^{\infty} d \lambda e^{-s \lambda} \Pi_{++}(\lambda)=\widehat{S}_{+}(s)+\frac{\omega(1-\rho)}{\nu^{b}} \widehat{I}(s),
$$

where

$$
\widehat{I}(s)=\int_{0}^{+\infty} d \lambda e^{-s \lambda} \int_{0}^{\lambda} d \lambda^{\prime} \int_{\lambda^{\prime}}^{\lambda} d \lambda^{\prime \prime} S_{+}\left(\lambda^{\prime}\right) S_{b}\left(\lambda^{\prime \prime}-\lambda^{\prime}\right) \Pi_{++}\left(\lambda-\lambda^{\prime \prime}\right) .
$$

By changing variables as

$$
\begin{gathered}
z_{1}=\lambda^{\prime} \\
z_{2}=\lambda^{\prime \prime}-z_{1}=\lambda^{\prime \prime}-\lambda^{\prime}, \\
z_{3}=\lambda-z_{2}-z_{1}=\lambda-\lambda^{\prime \prime},
\end{gathered}
$$

we can rewrite the integral as

$$
\widehat{I}(s)=\int_{0}^{+\infty} d z_{1} e^{-s z_{1}} S_{+}\left(z_{1}\right) \int_{0}^{+\infty} d z_{2} e^{-s z_{2}} S_{b}\left(z_{2}\right) \int_{0}^{+\infty} d z_{3} e^{-s z_{3}} \Pi_{++}\left(z_{3}\right),
$$

i.e.

$$
\widehat{I}(s)=\widehat{S}_{b}(s) \widehat{S}_{+}(s) \widehat{\Pi}_{++}(s) .
$$

If we combine Eq. (5.51) and Eq. (5.52) together we finally obtain

$$
\widehat{\Pi}_{++}(s)=\frac{\widehat{S}_{+}(s)}{1-\frac{\omega(1-\rho)}{\nu^{b}} \widehat{S}_{b}(s) \widehat{S}_{+}(s)},
$$

and, by observing that $\widehat{S}_{+}(s)=(1+s)^{-1}$ and $\widehat{S}_{b}(s)=\left(\frac{\rho+\omega}{\nu^{b}}+s\right)^{-1}$,

$$
\widehat{\Pi}_{++}(s)=\frac{\frac{\omega(1-\rho)}{\nu^{b}}+s}{s^{2}+\left(1+\frac{\rho+\omega}{\nu^{b}}\right) s-\frac{\omega(1-\rho)}{\nu^{b}}},
$$

If we now define $\widehat{f}(s)=\frac{\rho+\omega}{\nu^{b}}+s$, and $\widehat{g}(s)=\left[s^{2}+\left(1+\frac{\rho+\omega}{\nu^{b}}\right) s-\frac{\omega(1-\rho)}{\nu^{b}}\right]^{-1}$, using the relation between Laplace transform and convolution we can write

$$
\begin{aligned}
\widehat{\Pi}_{++}(s)=\widehat{f}(s) \widehat{g}(s) & =\int_{0}^{+\infty} d \lambda e^{-s \lambda} \int_{0}^{\lambda} d \lambda^{\prime} f\left(\lambda^{\prime}\right) g\left(\lambda-\lambda^{\prime}\right) \\
& =\int_{0}^{+\infty} d \lambda e^{-s \lambda} \Pi_{++}(\lambda),
\end{aligned}
$$


where $f$ and $g$ are the inverse Laplace transforms of $\widehat{f}$ and $\widehat{g}$, respectively. Therefore

$$
\begin{aligned}
\Pi_{++}(\lambda)=\int_{0}^{\lambda} d \lambda^{\prime}\left[\frac{\rho+\omega}{\nu^{b}} \delta\left(\lambda^{\prime}\right)+\frac{d}{d \lambda^{\prime}} \delta\left(\lambda^{\prime}\right)\right] \\
\quad \times \frac{e^{-\frac{1}{2}\left(1+\frac{\rho+\omega}{\nu^{b}}\right)\left(\lambda-\lambda^{\prime}\right)}\left[e^{\frac{1}{2} \Delta\left(\lambda-\lambda^{\prime}\right)}-e^{-\frac{1}{2} \Delta\left(\lambda-\lambda^{\prime}\right)}\right]}{\Delta}
\end{aligned}
$$

and finally, by integrating last integral by parts and going back to $\lambda \rightarrow \lambda-\lambda_{0}$, we obtain

$$
\begin{aligned}
\Pi_{++}\left(\lambda \mid \lambda_{0}\right)=e^{-\frac{1}{2}\left(1+\frac{\rho+\omega}{\nu^{b}}\right)\left(\lambda-\lambda_{0}\right)} & \\
& \times\left[\frac{\Delta+\frac{\rho+\omega}{\nu^{b}}-1}{2 \Delta} e^{\frac{1}{2} \Delta\left(\lambda-\lambda_{0}\right)}+\frac{\Delta-\frac{\rho+\omega}{\nu^{b}}+1}{2 \Delta} e^{-\frac{1}{2} \Delta\left(\lambda-\lambda_{0}\right)}\right],
\end{aligned}
$$

and similarly

$$
\Pi_{+b}\left(\lambda \mid \lambda_{0}\right)=\frac{\omega}{\nu^{b} \Delta} e^{-\frac{1}{2}\left(1+\frac{\rho+\omega}{\nu^{b}}\right)\left(\lambda-\lambda_{0}\right)}\left[e^{\frac{1}{2} \Delta\left(\lambda-\lambda_{0}\right)}-e^{-\frac{1}{2} \Delta\left(\lambda-\lambda_{0}\right)}\right] .
$$

Furthermore, with the same calculation we can as well compute

$$
\Pi_{b+}\left(\lambda \mid \lambda_{0}\right)=\frac{1-\rho}{\Delta} e^{-\frac{1}{2}\left(1+\frac{\rho+\omega}{\nu^{b}}\right)\left(\lambda-\lambda_{0}\right)}\left[e^{\frac{1}{2} \Delta\left(\lambda-\lambda_{0}\right)}-e^{-\frac{1}{2} \Delta\left(\lambda-\lambda_{0}\right)}\right],
$$

and

$$
\begin{aligned}
\Pi_{b b}\left(\lambda \mid \lambda_{0}\right)=e^{-\frac{1}{2}\left(1+\frac{\rho+\omega}{\nu^{b}}\right)\left(\lambda-\lambda_{0}\right)} & \\
& \times\left[\frac{\Delta-\frac{\rho+\omega}{\nu^{b}}+1}{2 \Delta} e^{\frac{1}{2} \Delta\left(\lambda-\lambda_{0}\right)}+\frac{\Delta+\frac{\rho+\omega}{\nu^{b}}-1}{2 \Delta} e^{-\frac{1}{2} \Delta\left(\lambda-\lambda_{0}\right)}\right] .
\end{aligned}
$$

Intriguingly, we observe that

$$
\Pi_{++}\left(\lambda \mid \lambda_{0}\right)=\frac{1}{\mathcal{M}_{n}} \mu^{+}\left(\lambda-\lambda_{0}\right)
$$

and

$$
\Pi_{b+}\left(\lambda \mid \lambda_{0}\right)=\frac{1}{\mathcal{M}_{n}} \beta\left(\lambda-\lambda_{0}\right)
$$

i.e. the probability to reach the length $\lambda-\lambda_{0}$ in the state $\sigma$ from the moment of the nucleation coincides with the length probability distribution in the state $\sigma$. This property of the system will be studied in a more general way and in detail in the Appendix A of this thesis. 


\subsection{Fast transport localizes actin at the surface}

In Section 5.2 we numerically studied a model with dynamics for both microtubules and actin, since an analytical solution did not seem to be reachable. To find an analytical solution for the model, we studied the case of fast actin diffusion, see Section 5.3. Even though that solution provided us information on the response of the distribution of microtubules to the change of their dynamics, it completely ignored the response of actin to the interaction between the two components of cytoskeleton.

Here, like we did in the previous section, in order to analitically describe the behaviour of actin due to the interaction with microtubules, we assume that the interaction does not change the dynamic properties of microtubules, i.e. does not change microtubule growing speed in the bound state.

Therefore, after some general considerations about the model equations presented in Section 5.2 - still with the assumption of no shrinking microtubules, we set $v^{b}=v^{+} \equiv v$ to analytically study the model both in 1 and in 3 -dimensions.

\section{Analytical approach}

If we sum Eqs. (5.6) and (5.7), we obtain

$$
D \nabla^{2} a(x)=\mathbf{v}^{\mathbf{b}} \cdot \nabla b(x),
$$

suggesting that the the two equations can be, in principle decoupled.

\section{1-dimensional case}

In the 1-dimensional case, the steady-state dynamic equations of the model (5.5-5.7) can be rewritten as

$$
\begin{gathered}
0=-v^{+} \frac{d}{d x} m(x)-r_{c} m(x)-r_{b} s m(x) a(x)+r_{u} b(x), \\
0=-v^{b} \frac{d}{d x} b(x)-\left(r_{c}+r_{u}\right) b(x)+r_{b} s m(x) a(x), \\
0=D \frac{d^{2}}{d x^{2}} a(x)-r_{b} s m(x) a(x)+\left(r_{u}+r_{c}\right) b(x),
\end{gathered}
$$

with boundary and normalization conditions

$$
\begin{gathered}
v^{+} m(0)=v^{+} m(R)+v^{b} b(R)+r_{c} M, \\
b(0)=0,
\end{gathered}
$$




$$
\begin{gathered}
\left.D \frac{d}{d x} a(x)\right|_{x=R}=v^{b} b(R), \\
\int_{0}^{R} d x[m(x)+b(x)]=M, \\
\int_{0}^{R} d x[a(x)+b(x)]=A .
\end{gathered}
$$

If we sum Eqs. (5.63) and (5.64), we obtain

$$
\frac{d}{d x}\left[D \frac{d}{d x} a(x)-v^{b} b(x)\right]=0
$$

which implies $D \frac{d}{d x} a(x)-v^{b} b(x)=$ const. for every $x \in[0, R]$. Therefore, from the condition (5.67) it follows that

$$
D \frac{d}{d x} a(x)=v^{b} b(x) \quad \forall x \in[0, R] .
$$

This equation has a very interesting consequence: indeed $b(x)$ is a distribution, and therefore, always positive. Thus, also the derivative of the free actin distribution is positive, meaning that $a(x)$ monotonically increases with the position $x$. Notice that, in the $\frac{D}{v^{b}} \gg R$ limit, Eq. (5.71) implies that $a(x) \simeq$ const., and the system reduces to the one previously studied in Section 5.3.

It follows that, if we want to control the slope and the steepness of the free actin distribution, we need to tune $\frac{D}{v^{b}}$, as it can be more clearly observed by integrating Eq. (5.71). Such an integration yields

$$
a(R)-a(0)=\frac{v^{b}}{D} B_{0}
$$

Figure 5.11 shows the increase in $a(R)-a(0)$ as $\frac{D}{v^{b}}$ falls. Notice that, for a very high value of $\frac{D}{v^{b}}$, i.e. in the fast diffusion limit, the free actin distribution is almost uniform. This figure shows the importance of a fast transport and a slow diffusion for the actin localization at the surface.

If we now plug Eq. (5.71) in Eq. (5.64) we obtain

$$
\frac{d^{2}}{d x^{2}} a(x)+\frac{r_{u}+r_{c}}{v^{b}} \frac{d}{d x} a(x)-\frac{r_{b} s}{D} m(x) a(x)=0,
$$

i.e. a second order differential equation for the distribution of free actin filaments, split from the distribution of bound actin. Unfortunately, given the coupling term $m(x) a(x)$, this equation cannot be solved analytically. 


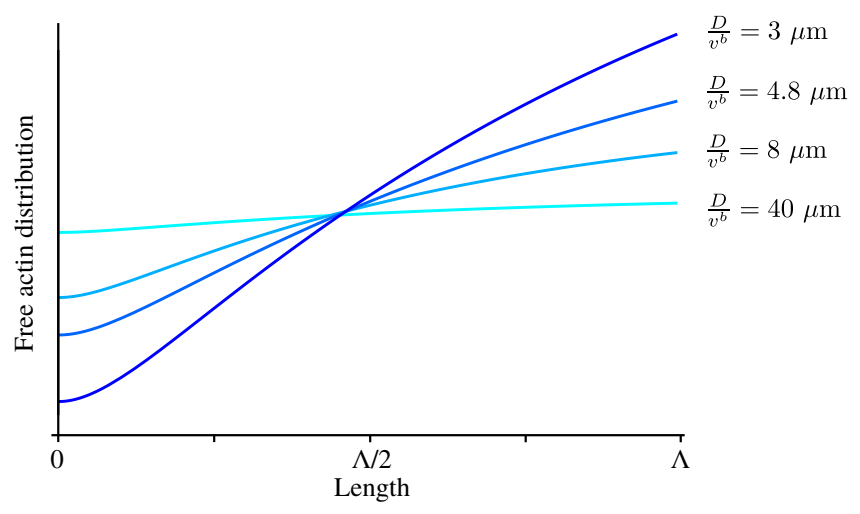

Figure 5.11: Distribution of free actin filaments for four different values of $\frac{D}{v^{b}}$.

\section{No change in microtubule growing speed}

Here, similarly to the case of fast diffusion for the actin, if we add Eq. (5.5) to Eq. (5.6), we obtain

$$
\frac{d}{d x}\left[v^{+} m(x)+v^{b} b(x)\right]=-r_{c}[m(x)+b(x)],
$$

which, in the $v^{+}=v^{b} \equiv v$ case, can be solved for $m(x)+b(x)$ to yield

$$
m(x)+b(x)=m_{0} e^{-\frac{r_{c}}{v} x},
$$

where the constant

$$
m_{0}=\frac{r_{c}}{v}\left(1-e^{-\frac{r_{c}}{v} R}\right)^{-1} M
$$

comes from the normalization condition $\int_{0}^{R} d x[m(x)+b(x)]=M$. Eq. (5.75) shows that, even in the case with diffusing actin, it is only the change in the growing speed that modifies the steady-state distribution for microtubule length. In other instances, Eq. (5.75) tells us that the changed length distribution is not dependent on whether or not the rate of switch from the unbound to the bound state is constant, as it depends only on the growing speed and the catastrophe rate.

In the scenario where $v^{+}=v^{b} \equiv v$ we can use Eq. (5.75) together with Eq. (5.71) to rewrite Eq. (5.73) in a closed form. Indeed

$$
a^{\prime \prime}(x)+\left[\frac{r_{u}+r_{c}}{v}+\frac{r_{b} s}{v} a(x)\right] a^{\prime}(x)-\frac{r_{b} s}{D} \frac{r_{c}}{v} M \frac{e^{-\frac{r_{c}}{v} x}}{1-e^{-\frac{r_{c}}{v} R}} a(x)=0 .
$$

Although Eq. (5.77) is still not analytically solvable, it highlights the fact that the system is described by four typical lengths: 
- the typical microtubule length

$$
\bar{l}=\frac{v}{r_{c}}
$$

- the transport length

$$
l_{t}=\frac{v}{r_{c}+r_{u}},
$$

that describes for how long an actin filament is transported by the tip of a microtubule,

- the free growth length

$$
l_{f}(x)=\frac{v}{r_{b} s a(x)},
$$

that describes how long a microtubule can grow before binding to an actin filament,

- the diffusion-transport length,

$$
l_{d t}=\frac{D}{v}
$$

that describes the competition between transport and diffusion, i.e. how well the free actin is redistributed in the cell after the detachment from a microtubule plus end.

Moreover, from the solution of Eq. (5.77) for $a(x)$ we can derive the solution for $b(x)$ through Eq. (5.71), and finally the solution for $m(x)$ from Eq. (5.75).

To better highlight the dependency of Eq. (5.77) on the relationship between dynamic parameters, we non-dimensionalize Eq. (5.77) by using $\bar{l}$ as the main length scale of the system. Thus, we define:

- position

$$
\lambda=\frac{x}{\bar{l}}=\frac{r_{c}}{v} x,
$$

- length of the cell

$$
\Lambda=\frac{R}{\bar{l}}=\frac{r_{c}}{v} R,
$$

- transport rate

$$
\gamma=\frac{\bar{l}}{l_{t}}=\frac{r_{u}+r_{c}}{r_{c}}
$$


- binding rate

$$
\eta=\frac{r_{b} s}{v}
$$

- transport-diffusion length

$$
\delta=\frac{l_{d t}}{\bar{l}}=\frac{r_{c} D}{v^{2}}
$$

- actin filament distribution

$$
\alpha=\frac{v}{r_{c}} a
$$

- growing microtubule distribution

$$
\mu=\frac{v}{r_{c}} m
$$

- re-scaled number of growing microtubules

$$
\mu_{0}=\frac{v}{r_{c}} m_{0}
$$

- bound microtubule distribution

$$
\beta=\frac{v}{r_{c}} b
$$

With the new variables, Eqs. (5.62-5.64) become

$$
\begin{gathered}
0=-\frac{d}{d \lambda} \mu(\lambda)-\mu(\lambda)-\eta \mu(\lambda) \alpha(\lambda)+(\gamma-1) \beta(\lambda), \\
0=-\frac{d}{d \lambda} \beta(\lambda)-\gamma \beta(\lambda)+\eta \mu(\lambda) \alpha(\lambda), \\
0=\delta \frac{d^{2}}{d \lambda^{2}} \alpha(\lambda)-\eta \mu(\lambda) \alpha(\lambda)+\gamma \beta(\lambda),
\end{gathered}
$$

whilst Eq. (5.77) becomes

$$
\alpha^{\prime \prime}(\lambda)+[\gamma+\eta \alpha(\lambda)] \alpha^{\prime}(\lambda)-\frac{\eta}{\delta} M \frac{e^{-\lambda}}{1-e^{-\Lambda}} \alpha(\lambda)=0 .
$$

This last equation shows that the whole system is governed by three independent parameters, namely the non-dimensional binding rate $\eta$, transport rate $\gamma$, and transport-diffusion length $\delta$. By tuning them we can control the spatial organization of both actin and microtubules. 


\section{Actin localization at the cell surface}

Here, we study the relationship between the different model parameters in order to have a strong localization of actin near the cell surface. A first requirement can be that the total actin concentration is monotonically increasing in $\lambda \in[0, \Lambda]$.

Using Eq. (5.71) and (5.79) we can observe that

$$
\frac{d}{d \lambda}[\alpha(\lambda)+\beta(\lambda)]=\left(\frac{1}{\delta}-\gamma\right) \beta(\lambda)+\eta \mu(\lambda) \alpha(\lambda) .
$$

The r.h.s. of last equation is positive if $\gamma \delta \leq 1$, or, going back to our dimensional quantities, if $l_{d t} \leq l_{t}$, i.e. if the diffusion-transport length is smaller than the transport length. In such a situation, the distribution of the actin does not have any maximum. Notice that the expression in Eq. (5.82) can be positive even if $\gamma \delta>1$, because of the second term on the right hand side. In this case, the positiveness of the expression is implied by

$$
\eta \mu(\lambda) \alpha(\lambda)>\left(\gamma-\frac{1}{\delta}\right) \beta(\lambda)
$$

or, if we express all our distributions in terms of $\alpha(\lambda)$,

$$
\left(\frac{\gamma \delta-1}{\alpha(\lambda)}-\eta \delta\right) \frac{d \alpha(\lambda)}{d \lambda}-\eta \mu_{0} e^{-\lambda}<0
$$

As we already observed, $\frac{d \alpha(\lambda)}{d \lambda}$ is always positive, and so is $\eta \mu_{0} e^{-\lambda}$. Therefore, Eq. (5.83) defines a necessary conditions that $\alpha(\lambda)$ needs to fulfill such that the total actin distribution does not have any maximum, that is

$$
\alpha(\lambda)>\frac{\gamma \delta-1}{\eta \delta}
$$

Although $\alpha(\lambda)$ parametrically depends on $\gamma, \delta$, and $\eta$, the last inequality can give an idea on how to tune the parameters in order to make inequality (5.83) hold. As the r.h.s. of the last inequality increases with $\gamma$ and $\delta$, while it decreases as $\eta$ grows, we can hypothesize e.g. that a high binding rate is linked to the fulfillment of inequality (5.83).

\section{3-dimensional case}

Here, we study whether or not the features highlighted by the model in the 1-dimensional case can be extended to 3 dimensions. 
If we add Eq. (5.6) to $x^{2}$ times Eq. (5.7), we obtain

$$
D \frac{d}{d x}\left[x^{2} \frac{d}{d x} a(x)\right]=v \frac{d}{d x}\left[x^{2} b(x)\right]
$$

that implies

$$
x^{2}\left[\frac{d}{d x} a(x)-\frac{v}{D} b(x)\right]=\text { const. }
$$

and, from the boundary condition (5.10)

$$
\frac{d}{d x} a(x)=\frac{v^{b}}{D} b(x) \quad \forall x \in[0, R],
$$

which means that the relation between the distributions of the free and the bound actin does not change when the dimension of the system increases.

Similarly, if we add Eqs. (5.5) to Eq. (5.6) we find

$$
\frac{d}{d x} x^{2}\left[v^{+} m(x)+v^{b} b(x)\right]=-r_{c} x^{2}[m(x)+b(x)] .
$$

This equation is solvable in the $v^{+}=v^{b} \equiv v$ case, and the solution is

$$
m(x)+b(x)=m_{0} \frac{e^{-\frac{r_{c}}{v} x}}{4 \pi x^{2}}
$$

where we used the normalization condition

$$
M=4 \pi \int_{0}^{R} d x x^{2}[m(x)+b(x)]
$$

to express the integration constant in terms of the model parameters, and were $m_{0}$ is defined by Eq. (5.76). This total distribution for the microtubules is the distribution that microtubules would have in case they were undergoing dynamic instability in a sphere of radius $R$ without any interaction with actin filaments. Then, Eq. (5.85) shows that in 3-dimensional case is the change in the growth speed of microtubules due to the interaction with actin, rather than the interaction itself, that causes the change in the distribution of microtubule plus end position, as well as in the 1-dimensional scenario.

In this $v^{+}=v^{b} \equiv v$ case, we can again write an expression for $a(x)$ in a closed-form by making use of Eqs. (5.84) and (5.85). Indeed

$$
a^{\prime \prime}(x)+\left[\frac{2}{x}+\frac{r_{u}+r_{c}}{v}+\frac{r_{b} s a(x)}{v}\right] a^{\prime}(x)-\frac{r_{b} s}{D} m_{0} \frac{e^{-\frac{r_{c}}{v} x}}{4 \pi x^{2}} a(x)=0 .
$$

This equation bears similarities with its 1-dimensional counterpart and, as well as in that case, from the (numerical) solution of Eq. (5.86) we can find the solution for $b(x)$ and $m(x)$ as well. 


\section{Probability to reach the surface}

In this section, we calculate the probability $\Pi_{i j}\left(x \mid x_{0}\right)$ that a microtubule with initial state $j$ and initial position $x_{0}$ reaches for the first time the position $x$ in the state $i$.

As we already did in Section 5.3, in order to calculate $\Pi_{i j}\left(x \mid x_{0}\right)$, we first need to define the survival probability $S_{\sigma}\left(t \mid t_{0}\right)$ of a microtubule in the state $\sigma$. In this case, since actin is no longer a constant background, the backward Kolmogorov equation (5.41) must be modified, in order to take into account the spatial organization of the free actin. Consider a microtubule initially in the growing state. Its survival probability in the growing state evolves as

$$
\frac{d}{d t} S_{+}\left(t \mid t_{0}\right)=-r_{c} S_{+}\left(t \mid t_{0}\right)-r_{b} s a[x(t)] S_{+}\left(t \mid t_{0}\right),
$$

with initial condition $S_{+}\left(t_{0} \mid t_{0}\right)=1$. However, since the growing speed of microtubules is constant, we can make the substitution $t=\frac{x}{v^{+}}$and $\frac{d}{d t}=v^{+} \frac{d}{d x}$. Therefore, previous equation becomes

$$
\frac{d}{d x} S_{+}\left(x \mid x_{0}\right)=-r_{c} S_{+}\left(x \mid x_{0}\right)-r_{b} s a(x) S_{+}\left(x \mid x_{0}\right),
$$

the solution of which is

$$
S_{+}\left(x \mid x_{0}\right)=\exp \left[-\frac{r_{c}}{v^{+}}\left(x-x_{0}\right)-\frac{r_{b} s}{v^{+}} \int_{x_{0}}^{x} d x^{\prime} a\left(x^{\prime}\right)\right] \equiv T_{-+}\left(x-x_{0}\right) T_{b+}\left(x \mid x_{0}\right),
$$

where we factorized the probability in the survival from the growing to the shrinking state, and from the growing to the bound state. Similarly, we can compute the survival probability in the bound state as

$$
S_{b}\left(x \mid x_{0}\right)=\exp \left[-\frac{r_{c}}{v^{b}}\left(x-x_{0}\right)-\frac{r_{u}}{v^{b}}\left(x-x_{0}\right)\right] \equiv T_{-b}\left(x-x_{0}\right) T_{+b}\left(x-x_{0}\right) .
$$

Notice that, since the detachment of the actin from the tip of a microtubule is not dependent on the concentration of actin, $S_{b}\left(x \mid x_{0}\right)$ coincides with the same function in the case of fast diffusion.

Now, we can finally calculate the probabilities

$$
\Pi_{++}\left(x \mid x_{0}\right)=S_{+}\left(x \mid x_{0}\right)+\int_{x_{0}}^{x} d x^{\prime} Q_{b+}\left(x^{\prime} \mid x_{0}\right) \Pi_{+b}\left(x \mid x^{\prime}\right),
$$

and

$$
\Pi_{+b}\left(x \mid x_{0}\right)=\int_{x_{0}}^{x} d x^{\prime} Q_{+b}\left(x^{\prime} \mid x_{0}\right) \Pi_{++}\left(x \mid x^{\prime}\right)
$$


where $Q_{\sigma \tau}$ is defined by Eq. (5.46). The meaning of Eq. (5.89) is the following: either a microtubule in the growing state at $x_{0}$ does not leave the growing state until it reaches the position $x$, or it switches to the bound state at a certain $x^{\prime} \in\left(x_{0}, x\right)$. From that moment it reaches $x$ in the growing state starting from $x^{\prime}$ in the bound, according to the probability $\Pi_{+b}$. If we plug Eq. (5.90) in (5.89), we find

$$
\begin{aligned}
\Pi_{++}\left(x \mid x_{0}\right)=S_{+} & \left(x \mid x_{0}\right) \\
& +\frac{r_{u} r_{b} s}{v^{+} v^{b}} \int_{x_{0}}^{x} d x^{\prime} \int_{x^{\prime}}^{x} d x^{\prime \prime} a\left(x^{\prime}\right) S_{+}\left(x^{\prime} \mid x_{0}\right) S_{b}\left(x^{\prime \prime} \mid x^{\prime}\right) \Pi_{++}\left(x \mid x^{\prime \prime}\right) .
\end{aligned}
$$

By changing the order of integration with the rule

$$
\left[x^{\prime} \in\left(x_{0}, x\right) \wedge x^{\prime \prime} \in\left(x^{\prime}, x\right)\right] \rightarrow\left[x^{\prime \prime} \in\left(x_{0}, x\right) \wedge x^{\prime} \in\left(x_{0}, x^{\prime \prime}\right)\right],
$$

we can rewrite last integral as a Volterra integral (Polyanin \& Manzhirov, Handbook of Integral Equation, 1998)

$$
\Pi_{++}\left(x \mid x_{0}\right)=S_{+}\left(x \mid x_{0}\right)+\frac{r_{u} r_{b} s}{v^{+} v^{b}} \int_{x_{0}}^{x} d x^{\prime} K\left(x^{\prime} \mid x_{0}\right) \Pi_{++}\left(x \mid x^{\prime}\right),
$$

where we defined $K$ as the kernel

$$
K\left(x^{\prime} \mid x_{0}\right)=\int_{x_{0}}^{x^{\prime}} d x^{\prime \prime} a\left(x^{\prime \prime}\right) S_{+}\left(x^{\prime \prime} \mid x_{0}\right) S_{b}\left(x^{\prime} \mid x^{\prime \prime}\right) .
$$

Similarly, we find

$$
\Pi_{+b}\left(x \mid x_{0}\right)=\frac{r_{u}}{v^{b}} \frac{J\left(x \mid x_{0}\right)}{a(x)}+\frac{r_{u} r_{b} s}{v^{+} v^{b}} \int_{x_{0}}^{x} d x^{\prime} J\left(x^{\prime} \mid x_{0}\right) \Pi_{+b}\left(x \mid x^{\prime}\right),
$$

where

$$
J\left(x \mid x_{0}\right)=a(x) \int_{x_{0}}^{x} d x^{\prime \prime} S_{+}\left(x \mid x^{\prime \prime}\right) S_{b}\left(x^{\prime \prime} \mid x_{0}\right) .
$$

With the same procedure, we can calculate the last two probabilities $\Pi_{b+}\left(x \mid x_{0}\right)$ and $\Pi_{b b}\left(x \mid x_{0}\right)$. We find

$$
\Pi_{b b}\left(x \mid x_{0}\right)=S_{b}\left(x \mid x_{0}\right)+\frac{r_{u} r_{b} s}{v^{+} v^{b}} \int_{x_{0}}^{x} d x^{\prime} J\left(x^{\prime} \mid x_{0}\right) \Pi_{b b}\left(x \mid x^{\prime}\right),
$$

and

$$
\Pi_{b+}\left(x \mid x_{0}\right)=\frac{r_{b} s}{v^{+}} K\left(x \mid x_{0}\right)+\frac{r_{u} r_{b} s}{v^{+} v^{b}} \int_{x_{0}}^{x} d x^{\prime} K\left(x^{\prime} \mid x_{0}\right) \Pi_{b+}\left(x \mid x^{\prime}\right) .
$$


Unfortunately without knowing the analytical expression of $a(x)$ we cannot solve the previous integral equations. However, since we can numerically calculate $a(x)$, we can use it to find a numerical solution for $\Pi_{\sigma \tau}$ as well. In this way, we could in principle check whether or not the probability of reaching a certain length in the state $\sigma$ coincides with the length probability distribution in the same state $\sigma$, as well as in the fast diffusion case.

Finally, as a check, notice that, if we set $s a(x)=1$, Eqs. (5.92-5.95) reduce to Eqs. (5.55-5.58).

\subsection{Conclusions}

In this chapter, we theoretically described a representation of a cell composed of only two interacting elements: microtubules and actin. In particular, we developed a mathematical model with microtubules nucleated at the centre of the cell and undergoing dynamic instability on the radial direction, and with diffusing actin. Starting from the experimental observation that the interaction between actin and microtubules had two effects - to change the dynamic properties of the microtubules, and to transport actin toward the surface by microtubule plus end, we explored the possible consequences of these effects.

In particular, we showed that the coexistence of two states for growing microtubules with two different growing speeds changes the steady-state distribution of the microtubule length, with respect to the steady-state length distribution in the Dogterom-Leibler model. This is the consequence of the diminished speed for microtubules bound to the actin, that results in a trapping mechanism of actin filaments close to the nucleation point.

Then, we showed that the key factor to win the competition against the trapping effect and localize actin filaments closer to the cell surface is the diffusion-transport length, defined as the fraction between the diffusion coefficient and the transport speed, i.e. $l_{d t}=D / v^{b}$. For short $l_{d t}$, indeed, we observed a significant monotonic increase of the actin distribution from the centre of the cell to the surface. The interpretation is that the motion of the actin after its release from a transporting microtubule is not fast enough to uniformly redistribute it in the cell volume before being captured again by a growing microtubule, resulting in a localization close to the surface. The observation that the diffusion coefficient of actin filaments is inversely proportional to the filaments length (Janmey et al., J. Biol. Chem., 1986), opens up the possibility of experimentally testing the outcomes of our theoretical predictions about the transport mechanism.

Interestingly, the comparison between the equations of the model and their dependency on model parameters in 1 and 3-dimensions does not reveal substantial 
qualitative differences between the two, except for the dilution terms that emerge from the increased dimension of the $3 \mathrm{D}$ case.

\subsection{Appendix}

\section{Actin transport in the case of a radial driving force}

Here, we want to briefly study the case in which the dynamics of microtubules is not altered by the interaction with the actin, and moreover, all microtubules act as a constant background that impulsively push actin toward the external part of the cell. Hence, in this case, we can consider actin undergoing a diffusive process with a drift force proportional to the microtubule density $m(x)$, and with reflective boundary conditions at both boundaries $x=0, R$.

This diffusive process for the actin is described by the Smoluchowski equation (Chandrasekhar, Rev. Mod. Phys, 1943). Since a general solution in the steadystate had been found only in the 1-dimensional case, in the rest of the section we will discuss only that very specific case.

Therefore, steady-state dynamic equations for microtubules and actin are

$$
0=-v \frac{d}{d x} m(x)-r_{c} m(x),
$$

and

$$
0=D \frac{d^{2}}{d x^{2}} a(x)-\frac{d}{d x}[k m(x) a(x)],
$$

where $k$ is a dimensional term proportional to the intensity of the drift force, such that the average velocity field that an actin filament is moving with at position $x$ is $V(x)=k m(x)$. Eqs. (5.96) and (5.97) are supported by the boundary and normalization conditions

$$
\begin{gathered}
M=\int_{0}^{R} d x m(x), \\
A=\int_{0}^{R} d x a(x),
\end{gathered}
$$

and

$$
\left.\frac{d}{d x} a(x)\right|_{x=0}-k m(0) a(0)=0=\left.\frac{d}{d x} a(x)\right|_{x=R}-k m(R) a(R) .
$$

From Eq. (5.96) and relative normalization condition it follows that

$$
m(x)=m_{0} e^{-\frac{r_{c}}{v} x}
$$


where

$$
m_{0}=\frac{r_{c}}{v}\left(1-e^{-\frac{r_{c}}{v} R}\right)^{-1} M .
$$

From Eq. (5.97), instead, it follows that

$$
D \frac{d}{d x} a(x)-k m(x) a(x)=\text { const. }
$$

i.e. a first order linear differential equation, the solution of which is

$$
a(x)=\exp \left[-\frac{k}{D} \frac{v}{r_{c}} m_{0} e^{-\frac{r_{c}}{v} x}\right]\left[c_{1}-c_{2} \frac{k}{D} \operatorname{Ei}\left(\frac{k}{D} \frac{v}{r_{c}} m_{0} e^{-\frac{r_{c}}{v} x}\right)\right]
$$

where $c_{1}$ and $c_{2}$ are integration constants and

$$
\operatorname{Ei}(z)=\int_{-\infty}^{z} d y \frac{e^{y}}{y}
$$

is the exponential integral.

Both reflecting boundary conditions at $x=0$ and $x=R$ imply

$$
c_{2}=0,
$$

whilst from the normalization condition

$$
A=\int_{0}^{R} d x a(x)=c_{1} \frac{v}{r_{c}}\left[\operatorname{Ei}\left(-\frac{k}{D} \frac{v}{r_{c}} m_{0}\right)-\operatorname{Ei}\left(-\frac{k}{D} \frac{v}{r_{c}} m_{0} e^{-\frac{r_{c}}{v} R}\right)\right],
$$

we obtain

$$
c_{1}=\frac{A}{\frac{v}{r_{c}}\left[\operatorname{Ei}\left(-\frac{k}{D} \frac{v}{r_{c}} m_{0}\right)-\operatorname{Ei}\left(-\frac{k}{D} \frac{v}{r_{c}} m_{0} e^{-\frac{r_{c}}{v} R}\right)\right]} .
$$

The final solution for the distribution of the actin is then

$$
a(x)=\frac{A}{\frac{v}{r_{c}}\left[\operatorname{Ei}\left(-\frac{k}{D} \frac{v}{r_{c}} m_{0}\right)-\operatorname{Ei}\left(-\frac{k}{D} \frac{v}{r_{c}} m_{0} e^{-\frac{r_{c}}{v} R}\right)\right]} \exp \left[-\frac{k}{D} \frac{v}{r_{c}} m_{0} e^{-\frac{r_{c}}{v} x}\right] .
$$

Figure 5.12 shows that, for the right choice of the force intensity $k$, the approximation of considering the transport of actin as the result of a velocity field that drives the filaments toward the surface of the cell is reasonable. 


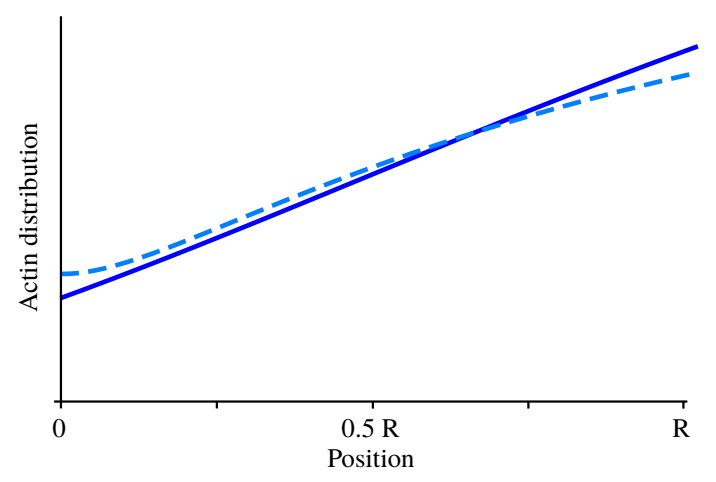

Figure 5.12: Distribution of free actin filaments from Eq. (5.99) (straight line), compared to the distribution of the original model (dashed line) in the case of $\frac{D}{v}=3 \mu \mathrm{m}$, see Figure 5.11. We choose $k=0.001 \mu \mathrm{m}^{2} \mathrm{~s}^{-1}$. 



\section{Conclusions}

In this thesis, we developed several stochastic models aimed at studying how the spatial organization of the cellular cytoskeleton is affected by the dynamics of its components. In particular, we studied two distinct mechanisms: the reorientation of the cortical microtubule array, and the transport of actin via microtubule tip.

\section{Reorientation of the cortical microtubule array}

The cortical microtubules in plant cells are a network of aligned microtubules localized close to the cell cortex. In the rapidly elongating phase of the cell cycle they are arranged transversely to the growth direction of the cell axis. In this way, they guide the transport and deposition of the building material for the cell cortex to expand. However, in vivo experiments have shown that, as a response to the exposure to blue light, cortical microtubules of dark-grown hypocotyls cell of Arabidopsis thaliana rapidly reorient from a transverse to a longitudinal direction to the long axis of the cell (Lindeboom et al., Science, 2013). Here, we set up two stochastic models of dynamic microtubules aimed at explaining the underlying mechanism behind the reorientation, at finding the key factors that enable it, and at understanding how a maintained reorientation can be achieved. 


\section{Microtubule tip stabilization promotes the reorientation}

The reorientation of the cortical microtubule array is a self-organizing process, consisting of a new population of longitudinal microtubules that replaces the initial transverse one. It is a process mediated by the severing protein katanin, that localizes at crossovers between differently oriented microtubules and preferentially severs the newer ones, resulting in the effective creation of a new microtubule. In most cases, newer microtubules are longitudinal. Typically, the newly-created plus end of a severed microtubule is destabilized and depolymerizes after a severing event. When the cell is illuminated with blue light, however, a fraction of these plus ends is stabilized and immediately grows (Lindeboom et al., Science, 2013). In this way, it has more opportunities to create other crossovers with other microtubules, and thus to be severed again and make the reorientation occur.

Further experiments have revealed that two key factors that enable the amplification of the number of longitudinal microtubules, and hence the reorientation, are: a high probability of rescue after severing, and a stable minus end (Lindeboom et al., J. Cell. Biol., 2018 - Nakamura et al., J. Cell Biol., 2018). While the first factor is thought to be responsible for the creation of a large number of crossovers, the second is thought to be correlated to the maintenance of crossovers for longer periods of time, with respect to a case where the minus end depolymerizes fast. Either way, the result is the occurrence of many severing events, and thus, an efficient and fast amplification. To test whether or not these factors play an important role for the amplification mechanism, in Chapter 2 we designed a model of longitudinal microtubules undergoing dynamic instability in a background of transverse microtubules. As we were interested only in understanding the reason of the initial amplification of the longitudinal array, in our model we ignored the dynamics of the transverse microtubules. This is a realistic choice only for the first time period of the reorientation process, namely the first 500 seconds. After that time, experiments revealed that the transverse array started to exhibit a considerable degree of suppression, and therefore its microtubule dynamics could not be ignored any longer.

By tuning the dynamic parameters of the model, we in turn isolated the response of the system to the change of the probability of rescue after severing and the dynamic instability parameters of microtubule minus end. Our simulations confirmed the experimental hypothesis that without either of those factors the amplification occurs more difficultly and, when it did, it is much slower than with a high probability of rescue after severing and a stable minus end. The observed occurrence of some amplification events even for fast depolymerizing microtubules at the minus end and low probability of rescue after severing, is linked to the observation that all microtubules under investigation were in the unbounded-growth 
regime. This means that, on average, their lifetime is infinite and therefore can be severed an increasing amount of times.

However, although we identified two key factors for a successful reorientation, some questions still remained unanswered. Firstly, longitudinal microtubules under study were in the unbounded-growth regime. Can the amplification occur in case of bounded-growth microtubules as well? We addressed this question in Chapter 3. Secondly, what happens to the system for time scales longer than 500 seconds? As here we are dealing with microtubules in the unbounded-growth regime that amplify in number, we can easily hypothesized that our proposed mechanism cannot be sustained by the cell for longer periods of time. We addressed this question in Chapter 4.

In Chapter 3 we analytically studied the computational model introduced in the previous chapter. Contrarily to the the previous case, however, we investigated the amplification mechanism for microtubules in the bounded-growth regime, the lifetime of which was finite. Our theoretical model predicted that in this regime, in order to have amplification, the probability of rescue after severing must be larger than a certain critical threshold defined by the dynamic parameters of longitudinal microtubules and the parameters of the background of transverse. With a combination of computer simulations and analytical calculations, we calculated the critical threshold. In order to do so, we introduced an approximate theory in which the possible number of crossovers for longitudinal microtubules was $a$ priori reduced to two. To that end, we developed an approximate technique to calculate the first passage time distribution for a microtubule to reach a relatively close target.

In conclusion, we showed that the regulation of microtubule ends dynamics plays a crucial role in the amplification phase of the longitudinal population of cortical microtubules. We showed that, in particular, two factors play a major role: a high probability of rescue after severing, as it increases the possibility for a microtubule to be severed more than one time, and a stable, non-depolymerizing minus end, as it does not let the possible severing spot disappear due to the shrinkage of the microtubule. Furthermore, we showed that, even though for unbounded-growth microtubules a high probability of rescue after severing is not strictly required in order to observe the amplification, it plays a very important role in making it occur fast. On the contrary, for bounded-growth microtubules, a high probability of rescue after severing is a necessary ingredient to enable the amplification mechanism, as it is required to be higher than a certain critical threshold. 


\section{Small advantage for longitudinal microtubules can explain a maintained reorientation}

In Chapters 2 and 3 we studied the first stage of the the reorientation process, and we identified the key factors for a rapid reorientation. However, the mechanisms as described in those chapters, cannot explain the later stage of the process, i.e. the maintenance of the newly-created longitudinal array. Indeed, the exponential amplification of microtubules implies that also the demand of tubulin to build them increases exponentially. Given that cells do not have enough dispersed tubulin in the cytoplasm to satisfy the request, it follows that the model proposed in Chapters 2 and 3 lacks of realism for long time periods. Therefore, in Chapter 4 we introduced a new model in which two different populations of microtubules - transverse and longitudinal, competed for a finite amount of tubulin. Our model revealed that the asymmetry induced by the experimentally observed preferential severing for longitudinal microtubules does not explain a sustained reorientation. Hence, we studied the effect of another asymmetry. We hypothesized that the growing speed of the longitudinal microtubules was slightly higher than that of the transverse microtubules. We observed that this difference in the growing speeds explains the maintained reorientation, whilst the preferential severing it is neither a necessary nor a sufficient ingredient for the reorientation to be maintained, although it helps the cortical microtubule array in reorienting rapidly. However, it is important to underline that with our result we do not state that the maintenance of the reorientation in cell is due to a difference in the growing speed between the two populations. Rather, we claim that if the difference existed, it would be enough to explain the mechanism.

In conclusion, we showed that a combination of a small advantage for the growth of a microtubule belonging to one population, and with a mechanism that rewards the overall length of a population with a higher affinity for new microtubule nucleation, causes the "victory" of that population when it competes against a second for the same amount of building material.

\section{Outlook}

Our study of the reorientation of the cortical microtubule array raised some questions and problems. Firstly, from the experiments it is possible to observe a correlation between preferential severing at a crossover, and age of the two microtubules involved in the crossover. We can speculate that, since new microtubules lay on top of old ones, the former might act as shields for the latter. However, at the moment, no experiments have been performed in order to understand the underlying mechanism behind the preferential severing, and therefore a combination 
of further experiments and analytical modelling should be designed to address this problem.

A second problem comes from the model proposed in Chapter 4. Indeed, there we assumed that the overall nucleation rate for microtubules is constant and split between possible nucleation in the two directions. However, the experiments showed that, after illuminating the cell with blue light, the number of nucleation events considerably depletes, in favor of a higher rate of microtubule creation due to the severing mechanism, see Figure 2.3. Mathematically, we can obtain this result only by changing the amount of the available tubulin in the free pool. Indeed, from Eq. 4.5 it follows that the less tubulin is available for microtubule growth, the lower is the overall nucleation rate. From Figure 4.11 we can observe that increasing the bias in the growing speed has an effect on the amount of free tubulin. This shows that, in principle, it is possible to control the availability of tubulin by acting on the other dynamic parameters of the model. Of course, further theoretical investigations should be aimed at confirming or refuting this hypothesis.

Therefore, a direct experimental observation of a difference in the growing speeds (or in any other dynamic parameter) of differently oriented microtubules could confirm not only the correctness of our prediction, but also the observed reduction in the number of nucleation events as the new longitudinal population takes over the initial transverse array. However, given that we showed that a small bias in the growth speed would be enough to explain the maintenance of the reoriented array, this speed difference could be, in principle, difficult to detect experimentally. Hence, in order to observe it, a very cautious interpretation of the data could be required, as well as a large amount of data points.

Nevertheless, it is convenient to point out that, even if a future experiment would refute our hypothesis that a difference growing speed exists, our model has the potential to be applied to other systems as well, together with its implications. For example, can be adapted to model the competition between different colonies of bacteria for space and resources (Hibbing et al., Nat. Rev. Microbiol., 2010), when one of the two colonies has a small advantage in obtaining the needed resources.

Besides the search for an experimental confirmation of our hypothesis on the underlying mechanism behind a maintained reorientation, our investigation also lacks a full, comprehensive computational verification. Indeed, although our model can explain the long time maintenance of the reoriented array, it is still too idealized to completely represent the dynamics of cortical microtubules in the cell. More precisely, in our model microtubules live in two non-physical spaces, with the possibility to interact only through the overall properties of the entire population they belong to. Thus, a computational approach that takes into account the geometry of the cell, the creation of crossovers, and the dynamics induced by the interaction between different microtubules, like e.g. severing events, induced 
catastrophes or zippering could be used to further prove or disprove our theoretical predictions. A computational framework that could be used to better simulate our system already exists. It has been initially developed by Tindemans (Tindemans et al., Front. Phys., 2014) and refined by Chakrabortty (Chakrabortty, 2017 Chakrabortty et al., Plos Comp. Biol., 2018), and consists of a set of triangular approximations of arbitrary areas of the cell surface, where microtubules undergo dynamic instability and can interact with each other for crossover creation events, as well as severing or zippering. This computational approach has the potential to give a computational explanation of our theoretical predictions. This model can already predict the reaching of an ordered state for microtubules that align in an oriented state while starting from an isotropic condition (Tindemans et al., Phys. Rev. Lett., 2010). A possible test for our theoretical prediction could consist of implementing the bias in the growing speed for microtubules randomly nucleated in a different, orthogonal direction with respect to that of the aligned microtubules, to check whether or not this bias can explain a reorientation of the array and its maintenance.

\section{Actin-microtubule interaction}

Although the interaction between actin and microtubules has been recognized as very important for many cellular mechanisms already sixty years ago (Abercrombie, Exp. Cell. Res., 1961 - Vasiliev et al., Development, 1970), no extensive studies have been made to give a description of the underlying mechanisms behind the interaction and its consequences. Here, starting from recently performed experiments (Alkemade et al., in preparation), we developed a theory to predict how the interaction between the two cytoskeletal components affects the spatial organization of the cytoskeleton. In particular, the main consequences of the TipAct-mediated interaction between actin filaments and microtubules, are two: a changed dynamics for the microtubules, the growing speed of which decreases while the catastrophe rate increments, and the transport of diffusing actin filaments by the tip of the microtubules.

\section{The interaction-induced change in microtubule dynamics qualitatively changes the steady-state distribution of microtubules}

Our theoretical model is based on the existence of two different growing speeds for microtubules, depending on whether they are growing freely or bound to an actin filament. In particular, it consists of dynamic microtubules undergoing dynamic 
instability in a three-dimensional cell, where actin filaments can diffuse, bind and unbind to the microtubule plus end. We predicted that the coexistence of two different speeds induces a change in the steady-state distribution of the microtubule length with respect to the exponential distribution of the Dogterom-Leibler model.

We showed that the length distribution of microtubules bound to actin filaments exhibits a maximum located close to the centre of the cell. This implies the existence of a trapping effect for actin filaments bound to microtubules that cannot diffuse anymore as they are stuck at microtubule tips. Intriguingly, we revealed that by tuning the dynamic parameters of microtubules that govern the position of the maximum we cannot significantly shift it towards the outer regions of the cell. As a consequence, acting on the microtubule dynamic parameters cannot avoid the trapping effect close to the centre. However, we observed that the diffusion coefficient plays an important role in reducing or amplifying the trapping effect, depending on whether the system was in a slow or fast diffusion regime, respectively. Therefore, even if such an effect is unavoidable, it can be reduced by the decrease of the diffusion coefficient of the diffusing actin.

Intriguingly, we noticed that the change in the steady-state behaviour for the length distribution of microtubules seems to be independent on the type of process that governs the binding and the unbinding of actin to and from the microtubule plus end.

\section{Fast transport by microtubule tip localizes actin close to the cell surface}

By making use of a combination of analytical derivations and numerical calculations, with our model we identified the key factors that control the localization of the actin filaments in the cell. Specifically, although we could not remove the trapping effect at the centre of the cell by changing the model parameters, mainly because of the large number of short microtubules that capture actin filaments in combination with the divergent distribution of freely growing microtubules in the centre due to the geometry of the system, we observed that a fast transport coupled to a slow diffusion causes localization of actin at the surface of the cell. This is a consequence of the fact that a slow diffusion does not allow actin to uniformly redistribute in the entire volume before binding again to a microtubule plus end, that transports it back close to the surface.

Finally, we showed that by assuming that there is no change in the microtubule dynamic parameters when they bind to actin filaments, it is possible to find a closed-form expression for the distribution of the latter. Even though this expression cannot be solved with analytical methods, its numerical solution allows us to find a numerical solution for the distribution of microtubules as well. 


\section{Outlook}

In our model, in order to find an analytically tractable solution, we have worked in a limit where rescues where not allowed. This is the case of the in vitro experiments we based our theoretical research on. However, in cells, the situation is more complicated, as microtubules can undergo rescues and be, in principle, also in the unbounded-growth regime. Therefore, the model we proposed could be in the future extended to account rescues as well. The introduction of rescue events, unfortunately, would give a further degree of complication to the already complicated set of Eqs. (5.1-5.4). For this reason, even if we can realistically expect to find only a numerical solution, we can anyway study whether or not our theoretical predictions would apply also in the full model with rescues.

A possible outlook for future experiments, instead, could be to verify if the presence of two possible speeds for growing microtubules that can switch from the growing to the bound state and viceversa, changes the steady-state microtubule length distribution. Such a verification is, in principle, possible. Indeed, in the experiments our model is based on, it has been observed that the growing speed of microtubules when in presence of TipAct is slower than in the control case without that cytolinker. Unfortunately, when in presence of TipAct, there is no evidence of a changed growing speed between the free growth state and the bound state. We can think that this is a consequence of the fact that the binding of TipAct on the tip of a microtubule via EB causes the change in the dynamic properties of the microtubule, rather than the binding with actin. Indeed, the experiments show that microtubules always exhibit TipAct localization at their plus end, and therefore, are always affected by the change that the cytolinker induces on their dynamics. An idea for future experiments could be to work with lower concentrations of TipAct. In this way, binding and unbinding events of TipAct from the plus end of microtubules could be detected, and the growing speed of microtubules in both states could be measured, as well as the length distribution of all microtubules. This could, in principle, verify our hypothesis that the coexistence of two different speeds causes a changed steady-state length distribution with respect to the Dogterom-Leibler model.

As regards the actin localization at the surface of the cell, although our results obtained in a three-dimensional confinement can be easily generalized to two dimensions, the quasi $2 \mathrm{D}$ in vitro experiments our model is based on cannot confirm the actin localization as a consequence of the transport. Indeed, in the experiments, microtubules are nucleated isotropically and from dispersed sites. Thus, they lack both a common nucleation site and a clear radial transport direction, with the consequence of redistributing actin over the entire area under observation. Nevertheless, some preliminary experimental observations of actin transport in 
three dimensions have been achieved with droplet experiments (Vendel et al., Methods in Molecular Biology, in press). Therefore, given that it has been observed that the diffusion coefficient of actin filaments can be tuned by acting on the filament length (Janmey et al., J. Biol. Chem., 1986), it is, in principle, possible to experimentally test our theoretical predictions about the actin localization as a consequence of the microtubule-based transport.

Finally, since here we focused on the analytical properties of the model, we still lack a comprehensive computational study of the system. Moreover, our model for a cell is very idealized, as many effects observed in the experiments or in in vivo observations have been ignored. As an example, the experiments our model is based on have revealed the existence of a diffusion mechanism for actin filaments on the lattice of microtubules, as well as they have detected some cases of backward transport of actin by depolymerizing microtubules. Furthermore, actin in the cell organizes in bundles and networks, while in our model we assumed that all actin filaments where freely diffusing non-interacting particles. A computational model would have less limitations than an analytical model in the implementation of an actin-actin interaction that forms the actin network. We can speculate that with this further ingredient, the actin localization at the surface would be even more pronounced. Indeed, an eventual filament that unbinds from the plus end of a microtubule next to the cortex would have a high probability of being "captured" by a second actin filament already there. The repetition of this mechanism could, in principle, create a trapping mechanism next to the cell cortex for actin filaments, resulting in the creation of the actin network. A computational framework able to verify this speculation and quantify this effect, could in principle raise intriguing (and philosophical) questions such as: is the actin network localized at the cell cortex because actin is nucleated there, or because it is brought by microtubules? 



\section{APPENDIX A}

\section{Probability distribution for a multi-state ageing process}

In both Chapters 2 and 5, we have studied the dynamics of microtubules with two possible different growing states, and we have seen that their co-existence with two different growing speeds leads to a changed steady-state distribution for the length of the microtubules with respect to the exponential distribution of the Dogterom-Leibler model. Moreover, we have shown that the assumption of constant rate to switch from one growing state to the other is a sufficient condition to obtain relations (5.59) and (5.60), i.e. the steady-state length distribution of microtubules in a specific state is directly proportional to the probability that a microtubule reaches a specific length in the same state.

Here, we extend the model to the case in which there are $N$ possible states. Given our goal of building a general theory, hereafter we will refer to individuals rather than microtubules, and to age of an individual in place of length of a microtubule.

\section{A.1 The model}

The model consists of new individuals born in the state $n_{0}$ with initial age $\tau=0$. At any time any individual in a state $n$ can switch to the state $k$ with rate $r_{n k}$ as well as it can die with death rate $d_{n}$. Finally, different states are associated to 
different ageing speeds $v_{n}$, i.e. individuals get older with different rates, depending on their state, see Figure A.1.

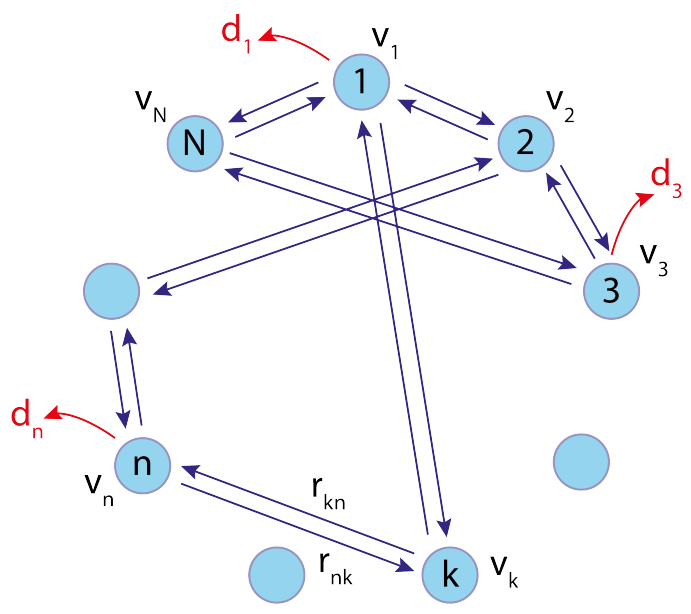

Figure A.1: Schematic of the model.

Therefore, if $p_{n}(t, \tau)$ is the probability distribution for the age of individuals in the state $n$ at time $t$, its dynamic equation is

$$
\frac{d}{d t} p_{n}(t, \tau)=-v_{n} \frac{d}{d \tau} p_{n}(t, \tau)-\sum_{k=1}^{N} r_{n k} p_{n}(t, \tau)-d_{n} p_{n}(t, \tau)+\sum_{k=1}^{N} r_{k n} p_{k}(t, \tau)
$$

with $r_{k k}=0, \forall k=1, \ldots, N$. Notice that the presence of the death rate $d_{n}$ in the previous equation ensures that the system eventually reaches the steady-state. Therefore, we can rewrite Eq. (A.1) for the steady-state case,

$$
v_{n} \frac{d}{d \tau} p_{n}(\tau)=-\sum_{k=1}^{N} r_{n k} p_{n}(\tau)-d_{n} p_{n}(\tau)+\sum_{k=1}^{N} r_{k n} p_{k}(\tau) .
$$

If we define $w_{k n}=\frac{r_{k n}}{v_{n}}$, and $Z_{n}=\frac{1}{v_{n}}\left(\sum_{k=1}^{N} r_{n k}+d_{n}\right)$, we can rewrite Eqs. (A.2) as

$$
\frac{d}{d \tau} p_{n}(\tau)=-Z_{n} p_{n}(\tau)+\sum_{k=1}^{N} w_{k n} p_{k}(\tau)
$$

or, in matrix form,

$$
\frac{d}{d \tau} \mathbf{p}(\tau)=\mathbf{L}^{T} \mathbf{p}(\tau)
$$


where

$$
\mathbf{L}=\left(\begin{array}{ccccc}
-Z_{1} & w_{12} & w_{13} & \cdots & w_{1 N} \\
w_{21} & -Z_{2} & w_{23} & \cdots & w_{2 N} \\
w_{31} & w_{32} & -Z_{3} & \cdots & w_{3 N} \\
\vdots & & & \ddots & \vdots \\
w_{N 1} & w_{N 2} & w_{N 3} & \cdots & -Z_{N}
\end{array}\right)
$$

Since all individuals are born in the initial state $n_{0}$, the boundary conditions are $p_{n}(0)=\mu \delta_{n n_{0}}$, where $\mu$ is the birth rate of new individuals. Thus, for $n=n_{0}$, from Eq. (A.3) it follows

$$
\left.\frac{d}{d \tau} p_{n_{0}}(\tau)\right|_{\tau=0}=-\frac{1}{v_{n_{0}}}\left[\sum_{k=1}^{N} r_{n_{0} k}+d_{n_{0}}\right] \mu=-Z_{n_{0}} \mu,
$$

and, for $n \neq n_{0}$,

$$
\left.\frac{d}{d \tau} p_{n}(\tau)\right|_{\tau=0}=\frac{r_{n_{0} n}}{v_{n}} \mu=w_{n_{0} n} \mu
$$

showing the non-surprising result that all $p_{n}$ s are initially increasing at $\tau=0$, except $p_{n_{0}}$ which is initially decreasing.

\section{A.2 Age distribution}

\section{Solution in Laplace space}

Let $n$ be the birth state of new individuals, i.e. $n=n_{0}$. Then, by Laplace transforming Eq. (A.3), we obtain

$$
\begin{gathered}
-\mu+s \hat{p}_{n}=-Z_{n} \hat{p}_{n}+\sum_{\substack{k_{1}=1 \\
k_{1} \neq n}}^{N} w_{k_{1} n} \hat{p}_{k_{1}}, \\
s \hat{p}_{k_{1}}=-Z_{k_{1}} \hat{p}_{k_{1}}+w_{n k_{1}} \hat{p}_{n}+\sum_{\substack{k_{2}=1 \\
k_{2} \neq n}}^{N} w_{k_{2} k_{1}} \hat{p}_{k_{2}} .
\end{gathered}
$$

The combination of the last two equations, yields

$$
\left(Z_{n}+s\right) \hat{p}_{n}=\mu+\sum_{\substack{k_{1}=1 \\ k_{1} \neq n}}^{N} \frac{w_{k_{1} n} w_{n k_{1}}}{Z_{k_{1}}+s} \hat{p}_{n}+\sum_{\substack{k_{1}, k_{2}=1 \\ k_{1}, k_{2} \neq n}}^{N} \frac{w_{k_{1} n} w_{k_{2} k_{1}}}{Z_{k_{1}}+s} \hat{p}_{k_{2}},
$$


and, if we iterate by replacing all $\hat{p}_{k_{j}} \mathrm{~s}$

$$
\left(Z_{n}+s-\sum_{i=1}^{+\infty} \sum_{k_{1}, \ldots, k_{i}=1}^{N} \frac{w_{k_{1} n} w_{k_{2} k_{1}} \ldots w_{n k_{i}}}{\left(Z_{k_{1}}+s\right) \ldots\left(Z_{k_{i}}+s\right)}\right) \hat{p}_{n}=\mu .
$$

By renaming the summation labels we finally obtain

$$
\hat{p}_{n}(s)=\frac{\mu}{Z_{n}+s-\sum_{i=1}^{+\infty} \sum_{k_{1}, \ldots, k_{i}} \frac{w_{n k_{1}} w_{k_{1} k_{2}} \ldots w_{k_{i} n}}{\left(Z_{k_{1}}+s\right) \ldots\left(Z_{k_{i}}+s\right)}} .
$$

Unfortunately, given that we are unable to rewrite the summation in Eq. (A.5) in a closed-form, we can not invert $\hat{p}_{n}(s)$ to find the distribution in the direct space. However, we can find some general solutions in specific cases, e.g., when the switches between states are cyclic.

\section{Cyclic switch}

If we assume that an individual in the state $n$ either switches to state $n+1$ or dies, with the possibility for an individual in the state $n=N$ to switch to state $n=1$, the matrix $\mathbf{L}$ from Eq. (A.4) becomes

$$
\mathbf{L}=\left(\begin{array}{ccccc}
-Z_{1} & w_{12} & 0 & \cdots & 0 \\
0 & -Z_{2} & w_{23} & \cdots & 0 \\
0 & 0 & -Z_{3} & \cdots & 0 \\
\vdots & & & \ddots & \vdots \\
w_{N 1} & 0 & 0 & \cdots & -Z_{N}
\end{array}\right)
$$

where $Z_{n}=\frac{r_{n, n+1}+d_{n}}{v_{n}}$. Then the secular equation $\operatorname{det}(\lambda-\mathbf{L})=0$ becomes

$$
\prod_{n=1}^{N}\left(\lambda+Z_{n}\right)-w_{12} w_{23} \cdots w_{N-1 N} w_{N 1}=0
$$

\section{Cyclic switch: general solution}

By Laplace transforming the equation $\frac{d \mathbf{p}(\tau)}{d \tau}=\mathbf{L}^{T} \mathbf{p}(\tau)$, with $\mathbf{L}$ defined by Eq. (A.6) and with initial condition $p_{k}(0)=\mu \delta_{k n}$, we obtain the set of equations

$$
\begin{aligned}
-\mu+s \hat{p}_{n} & =-Z_{n} \hat{p}_{n}+w_{n-1, n} \hat{p}_{n-1}, \\
s \hat{p}_{k} & =-Z_{k} \hat{p}_{k}+w_{k-1, k} \hat{p}_{k-1},
\end{aligned}
$$


the solution of which is

$$
\hat{p}_{n}(s)=\frac{\mu}{\prod_{k=1}^{N}\left(s+Z_{k}\right)-\prod_{k=1}^{N} w_{k, k+1}} .
$$

We notice that the denominator of Eq. (A.9) is such that

$$
\prod_{k=1}^{N}\left(s+Z_{i}\right)-\prod_{k=1}^{N} w_{k, k+1}=\operatorname{det}(s-\mathbf{L})=\prod_{k=1}^{N}\left(s-\lambda_{k}\right),
$$

where $\lambda_{k}$ are the zeroes of the polynomial det $(s-\mathbf{L})$. However, the zeroes of $\operatorname{det}(s-\mathbf{L})$ are the solutions of the secular equation $\operatorname{det}(\lambda-\mathbf{L})=0$ of previous section, hence $\lambda_{k}$ are the eigenvalues of $\mathbf{L}$. Therefore, if we have $M$ distinct eigenvalues $\lambda_{k_{j}}$, with respective algebraic multiplicity $a_{j_{k}}$, Eq. (A.9) becomes, in direct space,

$$
p_{n}(\tau)=\mu \sum_{j_{k}=1}^{M} q_{a_{j_{k}}, n}(\tau) e^{\lambda_{j_{k}} \tau}
$$

where $q_{a_{j_{k}, n}}(\tau)$ is a polynomial of degree $a_{j_{k}}$ in $\tau$.

Similarly, for $k \neq n$, we find

$$
\hat{p}_{k}(s)=\mu\left(\prod_{i=n}^{k-1} w_{i, i+1}\right) \frac{\left(s+Z_{1}\right) \cdots\left(s+Z_{k-1}\right)\left(s+Z_{n+1}\right)\left(s+Z_{N}\right)}{\operatorname{det}(s-\mathbf{L})},
$$

and therefore

$$
p_{k}(\tau)=\mu \sum_{j_{i}=1}^{M} q_{a_{j_{i}}, k}(\tau) e^{\lambda_{j_{i}} \tau}
$$

More compactly,

$$
\mathbf{p}(\tau)=\mu \sum_{j_{i}=1}^{M} \mathbf{q}_{a_{j_{i}}}(\tau) e^{\lambda_{j_{i}} \tau}
$$

\section{A.3 Age probability}

Here, we calculate the probability that an individual born in $n_{0}=n$ is in state $k=1, \ldots, N$ at age $\tau$.

In order to do so, we first define the survival probability of an individual from age $\tau_{0}$ to age $\tau$ in the state $k$ as

$$
S_{k}\left(\tau \mid \tau_{0}\right)=e^{-Z_{k}\left(\tau-\tau_{0}\right)} .
$$


We notice that $S_{k}\left(\tau \mid \tau_{0}\right)=S_{k}\left(\tau-\tau_{0}\right)$. Let $n_{0}=n$ be the initial state. Then, the probability that an individual reaches age $\tau$ starting from $\tau_{0}=0$ in state $k$ is

$$
\begin{gathered}
\Pi_{n n}(\tau)=S_{n}(\tau)+\int_{0}^{\tau} d \tau_{1} \sum_{\substack{k=1 \\
k \neq n}}^{N} w_{n k} S_{n}\left(\tau_{1}\right) \Pi_{n k}\left(\tau-\tau_{1}\right), \\
\Pi_{n k}(\tau)=\int_{0}^{\tau} d \tau_{1} S_{k}\left(\tau_{1}\right)\left[w_{k n} \Pi_{n n}\left(\tau-\tau_{1}\right)+\sum_{\substack{j=1 \\
j \neq n}}^{N} w_{k j} \Pi_{n j}\left(\tau-\tau_{1}\right)\right] .
\end{gathered}
$$

By combining iteratively the two equations, we obtain

$$
\begin{aligned}
\Pi_{n n}(\tau)= & S_{n}(\tau)+\int_{0}^{\tau} d \tau_{1} \int_{\tau_{1}}^{\tau} d \tau_{2} S_{n}\left(\tau_{1}\right) \sum_{k_{1}} w_{n k_{1}} w_{k_{1} n} S_{k_{1}}\left(\tau_{2}\right) \Pi_{n n}\left(\tau-\tau_{2}\right) \\
& +\int_{0}^{\tau} d \tau_{1} \int_{\tau_{1}}^{\tau} d \tau_{2} \int_{\tau_{2}}^{\tau} d \tau_{3} S_{n}\left(\tau_{1}\right) \\
& \quad \times \sum_{k_{1}, k_{2}} w_{n k_{1}} w_{k_{1} k_{2}} w_{k_{2} n} S_{k_{1}}\left(\tau_{2}\right) S_{k_{2}}\left(\tau_{3}\right) \Pi_{n n}\left(\tau-\tau_{3}\right) \\
& +\cdots \quad \int_{\tau_{i-1}}^{\tau} d \tau_{i} S_{n}\left(\tau_{1}\right) \\
& +\int_{0}^{\tau} d \tau_{1} \ldots w_{n k_{1}} \ldots w_{k_{i-1} n} S_{k_{1}}\left(\tau_{2}\right) \ldots S_{k_{i-1}}\left(\tau_{i}\right) \Pi_{n n}\left(\tau-\tau_{i}\right) \\
& \times \sum_{k_{1}, \ldots, k_{i-1}} w
\end{aligned}
$$

\section{Solution in Laplace space}

We take the Laplace transform of one integral in Eq. (A.13), i.e.

$$
\begin{array}{r}
\hat{I}_{i}=\int_{0}^{+\infty} d \tau e^{-s \tau} \int_{0}^{\tau} d \tau_{1} \int_{\tau_{1}}^{\tau} d \tau_{2} \ldots \int_{\tau_{i-1}}^{\tau} d \tau_{i} S_{n}\left(\tau_{1}\right) S_{k_{1}}\left(\tau_{2}\right) \ldots S_{k_{i-1}}\left(\tau_{i}\right) \\
\times \Pi_{n n}\left(\tau-\tau_{i}\right) .
\end{array}
$$


We first change the order of integration,

$$
\begin{array}{r}
\hat{I}_{i}=\int_{0}^{+\infty} d \tau_{1} \int_{\tau_{1}}^{+\infty} d \tau_{2} \ldots \int_{\tau_{i-1}}^{+\infty} d \tau_{i} \int_{\tau_{i}}^{+\infty} d \tau e^{-s \tau} S_{n}\left(\tau_{1}\right) S_{k_{1}}\left(\tau_{2}\right) \ldots S_{k_{i-1}}\left(\tau_{i}\right) \\
\times \Pi_{n n}\left(\tau-\tau_{i}\right),
\end{array}
$$

and after we substitute the integration variables with the rule

$$
\begin{gathered}
x_{1}=\tau_{1}, \\
x_{2}=\tau_{2}-x_{1}, \\
\cdots \\
x_{i}=\tau_{i}-\left(x_{1}+\cdots+x_{i-1}\right), \\
x=\tau-\left(x_{1}+\cdots+x_{i}\right) .
\end{gathered}
$$

This substitution yields

$$
\begin{aligned}
\hat{I}_{i}=\int_{0}^{+\infty} d x_{1} e^{-s x_{1}} S_{n}\left(x_{1}\right) \int_{0}^{+\infty} d x_{2} e^{-s x_{2}} S_{k_{1}}\left(x_{2}\right) \cdots & \int_{0}^{+\infty} d x_{i} e^{-s x_{i}} S_{k_{i}}\left(x_{i}\right) \\
& \times \int_{0}^{+\infty} d x e^{-s x} \Pi_{n n}(x),
\end{aligned}
$$

or

$$
\hat{I}_{i}=\hat{S}_{n} \hat{S}_{k_{1}} \cdots \hat{S}_{k_{i}} \hat{\Pi}_{n n},
$$

with $\hat{S}_{j}=\frac{1}{Z_{j}+s}$. We plug this result in Eq. (A.13) to obtain

$$
\hat{\Pi}_{n n}=\hat{S}_{n}+\hat{S}_{n} \sum_{i=1}^{+\infty} \sum_{\substack{k_{1}, \ldots, k_{i}=1 \\ k_{1}, \ldots, k_{i} \neq n}}^{N} w_{n k_{1}} w_{k_{1} k_{2}} \ldots w_{k_{i} n} \hat{S}_{k_{1}} \cdots \hat{S}_{k_{i}} \hat{\Pi}_{n n}
$$

from which

$$
\hat{\Pi}_{n n}(s)=\frac{1}{Z_{n}+s-\sum_{i=1}^{+\infty} \sum_{k_{1}, \ldots, k_{i}} \frac{w_{n k_{1}} w_{k_{1} k_{2}} \ldots w_{k_{i} n}}{\left(Z_{k_{1}}+s\right) \ldots\left(Z_{k_{i}}+s\right)}} .
$$

Since Eqs. (A.5) and (A.18) coincide in the Laplace space, by the Lerch's theorem (Lerch, 1903) the two functions are the same function also in the direct space $\tau$, i.e.

$$
p_{n}\left(\tau-\tau_{0}\right)=\mu \Pi_{n n}\left(\tau \mid \tau_{0}\right) .
$$




\section{Bibliography}

[1] M. Abercrombie. "The bases of the locomotory behaviour of fibroblasts." In: Experimental cell research Suppl 8 (1961), pp. 188-98.

[2] M Abramowitz and I. A. Stegun. "Handbook of mathematical functions with formulas, graphs, and mathematical tables". In: Dover Books on Advanced Mathematics, New York: Dover, Corrected edition, edited by Abramowitz, Milton; Stegun, Irene A. (1965).

[3] R. C. Adikes et al. "Control of microtubule dynamics using an optogenetic microtubule plus end-F-actin cross-linker". In: The Journal of Cell Biology 217.2 (Feb. 2018), pp. 779-793.

[4] B. Alberts et al. Molecular biology of the cell. Garland Science, 2002.

[5] C. Alkemade, M. Dogterom, and G. Koenderink. "in preparation". In: ().

[6] C. Alkemade et al. "in preparation". In: ().

[7] P. W. Baas et al. "Stability properties of neuronal microtubules". In: $C y$ toskeleton 73.9 (Sept. 2016), pp. 442-460.

[8] J. Al-Bassam et al. "CLASP Promotes Microtubule Rescue by Recruiting Tubulin Dimers to the Microtubule". In: Developmental Cell 19.2 (Aug. 2010), pp. 245-258.

[9] D. J. Bicout. "Green's functions and first passage time distributions for dynamic instability of microtubules". In: Physical Review E 56.6 (Dec. 1997), pp. 6656-6667. 
[10] S. R. Bisgrove, W. E. Hable, and D. L. Kropf. "+TIPs and Microtubule Regulation. The Beginning of the Plus End in Plants". In: Plant Physiology 136.4 (Dec. 2004), pp. 3855-3863.

[11] D. H Burk et al. A Katanin-like Protein Regulates Normal Cell Wall Biosynthesis and Cell Elongation. Tech. rep. 2001, pp. 807-827.

[12] B. Chakrabortty. "Plant cortical microtubule dynamics and cell division plane orientation". PhD thesis. 2017.

[13] B. Chakrabortty et al. "A computational framework for cortical microtubule dynamics in realistically shaped plant cells". In: PLoS Computational Biology 14.2 (Feb. 2018). Ed. by Qing Nie, e1005959.

[14] S. Chandrasekhar. "Stochastic Problems in Physics and Astronomy". In: Reviews of Modern Physics 15.1 (Jan. 1943), pp. 1-89.

[15] H. Chen et al. "The role of microtubule actin cross-linking factor 1 (MACF1) in the Wnt signaling pathway." In: Genes 8 development 20.14 (July 2006), pp. 1933-45.

[16] D. Chrétien et al. "Lattice defects in microtubules: protofilament numbers vary within individual microtubules." In: The Journal of cell biology 117.5 (June 1992), pp. 1031-40.

[17] E. N. Cytrynbaum et al. "Computational model of dynein-dependent selforganization of microtubule asters." In: Journal of cell science 117.Pt 8 (Mar. 2004), pp. 1381-97.

[18] E. E. Deinum, S. H. Tindemans, and B. M Mulder. "Taking directions: the role of microtubule-bound nucleation in the self-organization of the plant cortical array". In: Physical Biology 8.5 (Oct. 2011), p. 056002.

[19] A. Desai and T. J. Mitchison. "Microtubule polymerization dynamics". In: Annual Review of Cell and Developmental Biology 13.1 (Nov. 1997), pp. 83117.

[20] R. Dixit and R. Cyr. "Encounters between Dynamic Cortical Microtubules Promote Ordering of the Cortical Array through Angle-Dependent Modifications of Microtubule Behavior". In: The Plant Cell 16.12 (Dec. 2004), pp. $3274-3284$.

[21] R. Dixit and R. Cyr. "The cortical microtubule array: from dynamics to organization." In: The Plant cell 16.10 (Oct. 2004), pp. 2546-52.

[22] M. Dogterom and G. H. Koenderink. "Actinâmicrotubule crosstalk in cell biology". In: Nature Reviews Molecular Cell Biology 20.1 (Jan. 2019), pp. 3854 . 
[23] M. Dogterom and S. Leibler. "Physical aspects of the growth and regulation of microtubule structures". In: Physical Review Letters 70.9 (Mar. 1993), pp. 1347-1350.

[24] D. W. Ehrhardt. "Straighten up and fly rightâmicrotubule dynamics and organization of non-centrosomal arrays in higher plants". In: Current Opinion in Cell Biology 20.1 (Feb. 2008), pp. 107-116.

[25] A. Elliott and S. L. Shaw. "Microtubule Array Patterns Have a Common Underlying Architecture in Hypocotyl Cells". In: Plant Physiology 176.1 (Jan. 2018), pp. 307-325.

[26] M. Erhardt et al. "The plant Spc98p homologue colocalizes with gammatubulin at microtubule nucleation sites and is required for microtubule nucleation." In: Journal of cell science 115.Pt 11 (June 2002), pp. 2423-31.

[27] S. Etienne-Manneville. "Actin and Microtubules in Cell Motility: Which One is in Control?" In: Traffic 5.7 (July 2004), pp. 470-477.

[28] B. S. Govindan and W. B. Spillman. "Steady states of a microtubule assembly in a confined geometry". In: Physical Review E 70.3 (Sept. 2004), p. 032901.

[29] K. E. Harms et al. "Pervasive density-dependent recruitment enhances seedling diversity in a tropical forest". In: Nature 404.6777 (Mar. 2000), pp. 493-495.

[30] J. J. Hartman and R. D. Vale. "Microtubule Disassembly by ATP-Dependent Oligomerization of the AAA Enzyme Katanin". In: Science 286.5440 (Oct. 1999), pp. 782-785.

[31] M. E. Hibbing et al. "Bacterial competition: surviving and thriving in the microbial jungle." In: Nature reviews. Microbiology 8.1 (Jan. 2010), pp. 15-25.

[32] F. Huber et al. "Cytoskeletal crosstalk: when three different personalities team up". In: Current Opinion in Cell Biology 32 (Feb. 2015), pp. 39-47.

[33] P. A. Janmey et al. "Structure and mobility of actin filaments as measured by quasielastic light scattering, viscometry, and electron microscopy." In: The Journal of biological chemistry 261.18 (June 1986), pp. 8357-62.

[34] J. M. Kollman et al. "Microtubule nucleating $\gamma$-TuSC assembles structures with 13-fold microtubule-like symmetry". In: Nature 466.7308 (Aug. 2010), pp. 879-882.

[35] K. J. (Keith James) Laidler. Chemical kinetics. 3rd ed. New York: Harper \& Row, 1987, p. 531.

[36] M. C. Ledbetter and K. R. Porter. "Morphology of Microtubules of Plant Cell". In: Science 144.3620 (May 1964), pp. 872-874. 
[37] M. Lerch. "Sur un point de la théorie des fonctions génératrices d'Abel". In: Acta Mathematica 27.0 (1903), pp. 339-351.

[38] J. J. Lindeboom et al. "A Mechanism for Reorientation of Cortical Microtubule Arrays Driven by Microtubule Severing". In: Science 342.6163 (Dec. 2013), p. 1245533.

[39] J. J. Lindeboom et al. "CLASP stabilization of plus ends created by severing promotes microtubule creation and reorientation". In: The Journal of Cell Biology 218.1 (Jan. 2019), pp. 190-205.

[40] R. B. Maccioni and V. Cambiazo. "Role of microtubule-associated proteins in the control of microtubule assembly". In: Physiological Reviews 75.4 (Oct. 1995), pp. 835-864.

[41] F. J. McNally and R. D. Vale. "Identification of katanin, an ATPase that severs and disassembles stable microtubules". In: Cell 75.3 (Nov. 1993), pp. 419-429.

[42] K. McNally et al. "Katanin controls mitotic and meiotic spindle length". In: The Journal of Cell Biology 175.6 (Dec. 2006), pp. 881-891.

[43] T. Mitchison and M. Kirschner. "Dynamic instability of microtubule growth". In: Nature 312.5991 (Nov. 1984), pp. 237-242.

[44] B. M. Mulder. "Microtubules interacting with a boundary: Mean length and mean first-passage times". In: Physical Review E 86.1 (July 2012), p. 011902.

[45] B. M. Mulder. "unpublished". In: ().

[46] T. Murata et al. "Microtubule-dependent microtubule nucleation based on recruitment of $\gamma$-tubulin in higher plants". In: Nature Cell Biology 7.10 (Oct. 2005), pp. 961-968.

[47] M. Nakamura et al. "SPR2 protects minus ends to promote severing and reorientation of plant cortical microtubule arrays." In: The Journal of cell biology 217.3 (Mar. 2018), pp. 915-927.

[48] R. Oleksy et al. "Flying foxes create extensive seed shadows and enhance germination success of pioneer plant species in deforested Madagascan landscapes." In: PloS one 12.9 (2017), e0184023.

[49] E. A. Papakonstanti and C. Stournaras. "Actin Cytoskeleton Architecture and Signaling in Osmosensing". In: Methods in enzymology. Vol. 428. 2007, pp. 227-240.

[50] A. Papoulis. Probability, random variables, and stochastic processes. McGrawHill, 1984, p. 576. 
Bibliography

[51] A. Polyanin, V. Zaitsev, and V. F. Zaitsev. Handbook of Nonlinear Partial Differential Equations. Chapman and Hall/CRC, Oct. 2003.

[52] M. Preciado López. "In vitro studies of actin-microtubule coordination". PhD thesis. 2015.

[53] M. Preciado López et al. "Actinâmicrotubule coordination at growing microtubule ends". In: Nature Communications 5.1 (Dec. 2014), p. 4778.

[54] L. Quarmby. "Cellular Samurai: katanin and the severing of microtubules." In: Journal of cell science 113 ( Pt 16).4 (Aug. 2000), pp. 2821-7.

[55] J. W. Reed et al. "Mutations in the gene for the red/far-red light receptor phytochrome B alter cell elongation and physiological responses throughout Arabidopsis development." In: The Plant Cell 5.2 (Feb. 1993), pp. 147-157.

[56] A. Roll-Mecak and R. D. Vale. "Making more microtubules by severing: a common theme of noncentrosomal microtubule arrays?" In: The Journal of cell biology 175.6 (Dec. 2006), pp. 849-51.

[57] A. Sampathkumar et al. "Live cell imaging reveals structural associations between the actin and microtubule cytoskeleton in Arabidopsis." In: The Plant cell 23.6 (June 2011), pp. 2302-13.

[58] A. Schmit. "Acentrosomal microtubule nucleation in higher plants." In: International review of cytology 220 (2002), pp. 257-89.

[59] S. L. Shaw, R. Kamyar, and D. W. Ehrhardt. "Sustained microtubule treadmilling in Arabidopsis cortical arrays." In: Science (New York, N.Y.) 300.5626 (June 2003), pp. 1715-8.

[60] J. R. Sider et al. "Direct observation of microtubule-f-actin interaction in cell free lysates." In: Journal of cell science 112 ( Pt 12) (June 1999), pp. 1947-56.

[61] S. E. Siegrist and C. Q. Doe. "Microtubule-induced cortical cell polarity". In: Genes 85 Development 21.5 (Mar. 2007), pp. 483-496.

[62] D. G. Sprugel. "Dynamic structure of wave-regenerated Abies balsamea forests in the north-eastern United States". In: Journal of Ecology (UK) (1976).

[63] A. Stuart and J. K. Ord. Distribution theory. Arnold, 1994.

[64] K. Sugimoto, R. E. Williamson, and G. O. Wasteneys. "Wall architecture in the cellulose-deficient rsw1 mutant of Arabidopsis thaliana: Microfibrils but not microtubules lose their transverse alignment before microfibrils become unrecognizable in the mitotic and elongation zones of roots". In: Protoplasma 215.1-4 (Mar. 2001), pp. 172-183. 
[65] R. P. Tas et al. "Differentiation between Oppositely Oriented Microtubules Controls Polarized Neuronal Transport". In: Neuron 96.6 (Dec. 2017), 12641271.e5.

[66] S. H. Tindemans. "Biomolecular design elements : cortical microtubules and DNA-coated colloids". PhD thesis. 2009.

[67] S. H. Tindemans, R. J. Hawkins, and B. M. Mulder. "Survival of the Aligned: Ordering of the Plant Cortical Microtubule Array". In: Physical Review Letters 104.5 (Feb. 2010), p. 058103.

[68] S. H. Tindemans and B. M. Mulder. "Microtubule length distributions in the presence of protein-induced severing". In: Physical Review E 81.3 (Mar. 2010), p. 031910.

[69] S. H. Tindemans et al. "Efficient event-driven simulations shed new light on microtubule organization in the plant cortical array". In: Frontiers in Physics 2 (Apr. 2014), p. 19.

[70] D. J. Tongway, C. Valentin, and J. Seghieri. Banded vegetation patterning in arid and semiarid environments : ecological processes and consequences for management. Springer, 2001, p. 251.

[71] E. Tortosa et al. "Microtubule-associated Protein 1B (MAP1B) Is Required for Dendritic Spine Development and Synaptic Maturation âi S". In: (2011).

[72] Ju. M. Vasiliev et al. "Effect of colcemid on the locomotory behaviour of fibroblasts". In: Development 24.3 (1970).

[73] K. J. A. Vendel et al. "in press". In: Methods in Molecular Biology. 2019.

[74] F. Verde et al. "Control of microtubule dynamics and length by cyclin Aand cyclin B-dependent kinases in Xenopus egg extracts." In: The Journal of cell biology 118.5 (Sept. 1992), pp. 1097-108.

[75] L. Vineyard et al. "Progressive transverse microtubule array organization in hormone-induced Arabidopsis hypocotyl cells." In: The Plant cell 25.2 (Feb. 2013), pp. 662-76.

[76] J. W. Vos, M. Dogterom, and A. M. C. Emons. "Microtubules become more dynamic but not shorter during preprophase band formation: A possible âsearch-and-captureâ mechanism for microtubule translocation". In: Cell Motility and the Cytoskeleton 57.4 (Apr. 2004), pp. 246-258.

[77] C. E. Walczak and R. Heald. "Mechanisms of Mitotic Spindle Assembly and Function". In: International review of cytology. Vol. 265. 2008, pp. 111-158. 
[78] C. Waterman-Storer et al. "Microtubules Remodel Actomyosin Networks in ii Cenopusi $_{\text {i i }}$ Egg Extracts via Two Mechanisms of F-Actin Transport". In: The Journal of Cell Biology 150.2 (July 2000), pp. 361-376.

[79] X. Wu, A. Kodama, and E. Fuchs. "ACF7 Regulates Cytoskeletal-Focal Adhesion Dynamics and Migration and Has ATPase Activity". In: Cell 135.1 (Oct. 2008), pp. 137-148.

[80] Q. Zhang et al. "Microtubule Severing at Crossover Sites by Katanin Generates Ordered Cortical Microtubule Arrays in Arabidopsis". In: Current Biology 24.8 (Apr. 2014), p. 917. 


\section{English summary}

In this thesis, we aimed at understanding how the dynamics of cytoskeletal components affects the spatial organization of the cytoskeleton. In particular, we focused on two systems: the cortical microtubule array, and an idealized cell consisting of only actin filaments and microtubules.

In the Introduction, we presented the main biological features of microtubules and the general model for dynamic microtubules we based the models we developed in the thesis on, and we outlined the research questions we addressed in the rest of the thesis.

In Chapter 2, we built a computational model for dynamic microtubules that reproduced the first phase of the reorientation of the cortical microtubule array observed the experiments. With our model, we identified two crucial dynamic factors of microtubules that enable the reorientation mechanism: a high probability of rescue after severing for newly-created plus end of microtubules, and a stable, non-depolymerizing microtubule minus end. Our findings confirmed the previous experimental observations, in which the presence of the two factors was hypothesized to be required for the reorientation in order to occur.

In Chapter 3, we theoretically performed an in-depth study of the model introduced in Chapter 2. In particular, we focused on the probability of rescue after severing for the newly-created plus end of a microtubule. To better understand the contribution of this parameter to the reorientation, we tuned it to study the response of the system. We observed that, in a certain area of the parameters space, the system exhibited a non-zero probability to reorient only if the probability of rescue after severing was larger than a critical threshold, defined by the remaining model parameters.

In Chapter 4, we studied the long time phase of the reorientation mechanism for the cortical microtubule array. More specifically, we built a second model where two populations of microtubules competed for the same amount of building material. We observed that a small advantage for the growth of microtubules of one of the two populations, made it prevail against the other. In this way, the cortical microtubule array could maintain the new orientation, as observed in the experiments.

In Chapter 5, we studied a different system in which dynamic microtubules interacted with diffusing actin filaments. Taking the cue from recently performed experiments, we built a stochastic model to make predictions on how microtubules and actin mutually influenced their spatial organization in a three dimensional confinement. Our model identified the factors that control this spatial organization, and in this way opened the possibility for future experimental tests on the predicted behaviour. 
In the Conclusions, we highlighted the main conclusions this work came to in the previous chapters. Furthermore, we presented some ideas for future research and investigations, that could be performed with a theoretical, computational, or experimental approach.

Finally, in the Appendix, we presented a stochastic model for individuals ageing in different possible states, with the possibility of switching from one state to another with constant rates. We showed that the model predicted an equality between the distribution of the age in a certain state and the probability for an individual to reach the same age in the state. 


\section{Acknowledgments}

Here we are, the most difficult section to write and the only one that everyone reads. I spent four years of fun and enthusiasm, when I had the possibility to increase my love and interest for science and research. Of course many people contributed to that, I just hope I won't forget anybody now.

First things first, Bela! If I have to summarize how was working with you in the last four years, I think I have a single word for it: fun! It has been amazing to have you as a supervisor, with your contagious optimism, positiveness, and kindness. I really want to thank you for your patience, and for guiding me through the last four years, from the moment of my interview (when you decided to start by offering me an "italian-like coffee"), to these last days of my stay at AMOLF. Hope we will work together again in the future.

Clearly this thesis couldn't be defended without the help of my two amazing paranymphs! Celine, collaborating with you has been a real pleasure, and being your friend an honor. Thanks for your kindness, and for offering your help so many times for so many different reasons. In particular, how not to mention your help in writing the propositions, i.e. how to turn something boring and complicated in something extremely funny (and I'm also still convinced that it was a good idea to avoid drinking the final whiskey). Mario, from arguing about the next song in the party playlist, to the late night sessions of Camel Up, or to almost passing out in the hot tub in Iceland... We really had so much fun time together, that I'm only sad that you're going to defend a few days before me, so that you will make me pay for everything I'll do to make fun of you during your party. Wish you the best for your academic career, and for your more important adventure with Ariadna and Olga. And by the way, I still want my avenge in Twilight Struggle, don't go thinking that I forgot it!

Fede, I'm very happy to have been your friend during these years, from the awkward moment in Veldhoven 2015 when we tried to read each other's name on the badge, to the less awkward moments of gossip, from the successful run dressed with ugly Christmas sweaters, to the less successful run when we've been stopped before the start... I'm really going to miss you, hope we will always keep in touch. Ale, I really don't have words to express how fun was to spend the last four years fooling around with you. We passed through so many funny moments together that it's difficult for me to find a specific anecdote. I could always count on you, and I'll always be thankful for that. Know that mi casa es su casa, for every time you're going to need a couch to sleep on after some late night party. Carolyn, thank you 
for always treating me with muffins, cakes, and candies, that you kept giving me as a gift after every single trip you have had, and for always offering your help anytime I needed. And yes, I know you're Dutch German, and I also know you are from Cologne Düsseldorf, but it was just funny to say you're not. Hope you will forgive me for that. Giulia, thanks for all dinners you prepared (we still have to prepare vongole together, remember), for every time we joked around, and for your text messages, usually a bit difficult to interpret. But also for all the times that you filmed me while I was unaware of that. I will get my avenge, know that! Lore, "if you want to go fast, you have to go slow. But on Monday morning ignoha gnaiii wooghfdh". From the first night I was aware of your existence because of the 3 a.m. Italian karaoke you organized at your place, to the morning when with Ale you tried to wake me up by smashing the mop against my window. Hope we will share this kind of things again in the future. Giorgio 'mbare, thanks for all the jokes and the funny moments we had together. And of course for letting me know about every single detail related to how to get the most out of dutch laws and benefits, and bureaucracy. And of course for bringing a descent amount of due italianity in every conversation. Mathijs, thanks for always being too active, always willing to organize some funny event and to kick out (literally, sometimes) the boredom that can arise in some rainy working days. And of course for your jokes, not funny at all but that somehow always made me laugh. Olga, thanks for all funny moments we had together, from our epic fail(s) while trying to hike in Iceland, to our challenging Codenames matches (you have been a great opponent). I wish you all the best wherever you'll go, with Mario and Ariadna. Hope we'll keep in touch! Harmen, thanks for all our nice and useful discussions, no matter if about science, politics, sports, or Argentinian steaks that we had. And of course for being unofficially part of Bela's group.

And again Anna and our senseless conversations and secret sharing, Laura and your funny "earthquaky" attitude, Kevin and our nerd conversations, Mareike, Masha, Giulia and our complaining about many different topics, Yao and our mathematical/computational conversations, Nicola and our fights about where polenta comes from. Give up with it, it's milanese! Then Giovanni JJ, and the Portuguese bar (and the time you take to roll your cigarettes), Andrea, and Andrea, Stephen, Victor, Ruben, and our "building dinners", it was nice to live below your flat, Fiona, Vanessa, although we have disagreement about actual italian singers, Eliane, Luci, you're also almost been adopted by the Theory of Biomolecular Matter group, Lennard, Jeffrey, Steffen, Joleen, Moritz and all nice parties we have been together, Laura, Rob, Chris, Judith, I promise I'll make fish again for you, Agata, and all our morning conversations from DUWO to AMOLF (mainly related to funny episodes occurred in Amsterdam while you were on holidays), Parisa, Cristina, Dzeni and the extensive amount of rubbish (yet amazing) conversations 
we had in the last four years, and for always being smiling, Michele, Jacopo, Pieter Rein and all our valuable discussions during our theory meetings, and all other people that I unforgivably forgot to mention.

Also, I would like to thank Maurits, Kim, Renu, Reza, Vladimir, Louis, and Marileen from the DogteromLab for the precious conversations about microtubules during the Synergy meetings. That helped a lot for the writing of this thesis. And also thanks for the nice time we spent in Heidelberg.

I want to thank every person who stayed in the office 0.17 , even for a short period: Anouk, Maarten and his butter in the coffee, Florian and his politically incorrect jokes (love them), Radek, Joost, Alex, and another couple of people whose name I completely forgot. And then my group, the Theory of Biomolecular Matter group: Emile, Felicio, Bart, Daniel, Poulomi, Gytis and your mathematical riddles, but also our morning greeting, Ramon and your daily updates on the brexit and your pointed irony, but also for all our inspirational conversations about basically every kind of topic, Bandan, Takis, Marieke, Lisa, Xavi, Faan and your cheerful attitude, Sanne, and Ireth: years ago I promised you a sentence in the acknowledgements, just don't remember why. I mean, there's plenty of reasons, but I don't remember what was that specifically about.

Then, I think it's fair to end this section with the first person I met in my AMOLF days: Melle. I really don't know how it is possible that you never killed me after all my pranks with your multiple mice, provocations, and after throwing to you my deadly eye drops so many times (I still want them back). It was really funny to be in the office with you and to share our opposite points of view about many different topics. Good luck with your new adventure!

And of course I can't write this section without mentioning all people who supported from $1000 \mathrm{~km}$ away. Thanks to Ale, Capu, Tado, Mirko, Losa, Gaggia, Jack, Vale, Campi, Tadde, Tsve, Fede, GM, Vale cumpa, Pode, Irene, Lele, Gabry, and Rix because not only you bear the cross of an amazing first name, but you also helped me a lot by designing the cover of this book.

Then, how not to mention mum, dad, and Serena. Thanks for supporting from far, not just for just these four years, but for the last 31, I should say. And of course for making me feel always welcome whenever I was in Italy to visit you.

Finally, Ele. Thanks for supporting and tolerating me for all these years, for coping with the distance and for letting me feel at home whenever you were here. But also for teaching me that spending one evening every now and then watching animal documentaries is as good as hanging out for drinks. Or that just walking in a park to take pictures to birds and flowers can be amazing if you do it with the right person. Or even that there couldn't be any such thing as the wrong moment to have aperitivo. And I probably should stop now, or I'd need at least other twenty pages to list all reasons why I'm grateful to you... Just thank you! 


\section{List of publications}

J. J. Lindeboom, M. Nakamura, M. Saltini, A. Hibbel, T. Ketelaar, A. M. Emons, A. Walia, J. C. Sedbrook, V. Kirik, B. M. Mulder, D. W. Ehrhardt. CLASP stabilization of plus ends created by severing promotes microtubule creation and reorientation. Journal of Cell Biology, 2018.

(chapter 2)

M. Nakamura, J. J. Lindeboom, M. Saltini, B. M. Mulder, D. W. Ehrhardt. SPR2 protects minus ends to promote severing and reorientation of plant cortical microtubule arrays. Journal of Cell Biology, 2018.

(chapter 2)

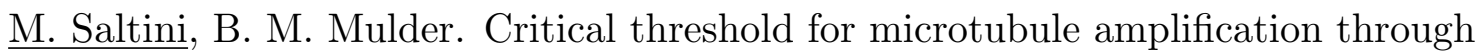
templated severing. arXiv preprint

(chapter 3)

M. Saltini, J. J. Lindeboom, D. W. Ehrhardt, B. M. Mulder. Reorientation of the cortical microtubule array in presence of finite tubulin pool. in preparation (chapter 4)

M. Saltini, B. M. Mulder. Microtubule-mediated actin transport and localization in three-dimensional confinement. in preparation (chapter 5) 
The research described in this thesis was financially supported by ERC 2013 Synergy Grant MODELCELL, and was performed at AMOLF, Science Park 104, 1098 XG, Amsterdam, the Netherlands.

Financial support from Wageningen University for printing this thesis is gratefully acknowledged.

Cover design by Marco Rissetto. 



\section{Propositions}

1. The regulation of tip stability of dynamic cortical microtubules is crucial for the success of the katanin-driven reorientation of the cortical microtubule array. (this thesis)

2. A small advantage in terms of growth of microtubules of one of two populations competing for the same pool of tubulin dimers, can cause a full, persistent reorientation of the cortical microtubule array.

(this thesis)

3. The history of the exact sciences shows that scientists should aim for beauty in conducting their research, rather than just focusing on applications.

4. Good mathematical modelling is like cooking: there are mandatory ingredients, but the real difference is made by the creative choice of additional ones by the modeller.

5. Since doing sports is an excellent way to release stress accumulated during office hours, employers should promote these activities for employees to foster a relaxed and, therefore, productive working environment.

6. Late night surfing on Wikipedia entails a trade-off: on the one hand it boosts the general knowledge of the surfer, yet on the other hand it deprives him/her of necessary sleep.

Propositions belonging to the thesis, entitled

Dynamic reorganization of microtubule networks: building the new by breaking down the old

Marco Saltini

Wageningen, 2 December 2019 